\title{
CONTRIBUTIONS TO DISCRETE-TIME METHODS FOR ROOM ACOUSTIC SIMULATION
}

Submitted in partial fulfillment of the requirements for the degree of Doctor of Philosophy

by

José Escolano-Carrasco

Technical University of Valencia

Valencia, Spain

July 2008 
Copyright (C) 2008 by José Escolano-Carrasco. All rights reserved. 
To Cari, for her love, understanding and patience. 


\section{Abstract}

The sound field distribution in a room is the consequence of the acoustic properties of radiating sources and the position, geometry and absorbing characteristics of the surrounding boundaries in an enclosure (boundary conditions). Despite there existing a consolidated acoustic wave theory, it is very difficult, nearly impossible, to find an analytical expression of the sound variables distribution in a real room, as a function of time and position. This scenario represents as an inhomogeneous boundary value problem, where the complexity of source properties and boundary conditions make that problem extremely hard to solve.

Room acoustic simulation, as treated in this thesis, comprises the algebraical approach to solve the wave equation, and the way to define the boundary conditions and source modeling of the scenario under analysis.

Numerical methods provide accurate algorithms for this purpose and among the different possibilities, the use of discrete-time methods arises as a suitable solution for solving those partial differential equations, particularized by some specific constrains. Together with the constant growth of computer power, those methods are increasing their suitability for room acoustic simulation. However, there exists an important lack of accuracy in the definition of some of these conditions so far: current frequency-dependent boundary conditions do not comply with any physical model, and directive sources in discrete-time methods have been hardly treated.

This thesis discusses about the current state-of-the-art of the boundary conditions and source modeling in discrete-time methods for room acoustic simulation, and it contributes some algorithms to enhance boundary condition formulation, in a locally reacting impedance sense, and source modeling in terms of directive sources under a defined radiation pattern. These algorithms have been particularized to some discrete-time methods such as the Finite Difference Time Domain and the Digital Waveguide Mesh.

Regarding to boundary conditions, two different approaches have been followed to achieve a locally reacting impedance boundary condition: one consisting into modify the reflection factor of the boundaries according with the plane wave angle of arrival and the other one, defining these boundaries in terms of the impedance, which is independent from this angle. Both methods have been implemented in a Digital Waveguide Mesh. From the 
results obtained, advantages and inconveniences are discussed throughout this thesis. Furthermore, based on these new methods, a locally reacting impedance boundary condition is proposed to the Finite Difference Time Domain method, in a similar fashion to the previous one.

On the new topic of directive sources for discrete-time methods, some new algorithms are proposed in this dissertation. In a first approach, a method which uses monopoles in order to be adapted to all discrete-time methods for a single frequency is proposed. Later, it has been extended for broadband simulations with a frequency-dependent directivity.

Keywords: Discrete-time methods, inhomogeneous boundary value problem, locally reacting impedance, room acoustic simulation, sound source modeling. 


\section{Resumen}

La distribución del campo sonoro en una habitación es la consecuencia de las propiedades ondulatorias de las fuentes acústicas y de la posición, geometría y propiedades absorbentes de las paredes que conforman los límites de la habitación (condiciones de contorno). Aunque existe una consolidada teoría acústica ondulatoria, es muy complejo, prácticamente imposible, encontrar una expresión analítica de la distribución de las variables acústicas en una sala real, en función de la posición y del tiempo. Esta situación debe tratarse como un problema no homogéneo de contorno, donde dada la complejidad de las condiciones de contorno y la definición de las fuentes, éstas hacen que el problema sea extremadamente complejo de resolver.

La simulación acústica de salas, tal como es tratada durante esta tesis, comprende la aproximación algebraica para resolver la ecuación de onda y el modo de definir los modelos de las fuentes y de las condiciones de contorno que caracterizan el escenario bajo análisis.

Los métodos numéricos proporcionan algoritmos precisos para este propósito, y entre las diferentes posibilidades, el uso de métodos en tiempo discreto surge como una solución adecuada para resolver esas ecuaciones en derivadas parciales, particularizadas para ciertas condiciones. Junto con el constante crecimiento de la capacidad de computación actual, crece la conveniencia de estos métodos para la simulación acústica de salas. Sin embargo, hasta ahora, existe una considerable falta de precisión en la definición de algunas de estas condiciones: las actuales condiciones de contorno dependientes de la frecuencia no cumplen ningún modelo físico, y las fuentes directivas en los métodos en tiempo discreto, apenas han sido tratadas.

Esta tesis trata sobre el estado del arte actual de las condiciones de contorno y la definición de fuentes sonoras en los métodos en tiempo discreto y contribuye con ciertos algoritmos para mejorar la formulación de las condiciones de contorno, en el sentido de impedancia de reacción local, y de los modelos de fuentes sonoras, en términos de directividad radiando conforme a un patrón de radiación determinado. Estos algoritmos han sido particularizados para el método de las Diferencias Finitas en el Dominio del Tiempo y para la Malla de Guías de Ondas Digitales.

Respecto a las condiciones de contorno, dos aproximaciones diferentes han sido propuestas con el fin de conseguir impedancias de reacción local: la 
primera consiste en modificar el factor de reflexión acorde con el ángulo de llegada de una onda plana; mientras que la otra solución propuesta, define las condiciones de contorno en términos de la impedancia, que es independiente del ángulo de llegada. Ambos métodos han sido implementados para la Malla de Guías de Ondas Digitales. A partir de los resultados obtenidos, se discuten las ventajas e inconvenientes a lo largo de esta tésis. Además, basado en estos nuevos métodos, se propone una definición de condiciones de contorno para el método de las diferencias finitas, de manera similar a los anteriores.

Respecto al nuevo tema propuesto de las fuentes directivas para métodos en tiempo discreto, una serie de algoritmos han sido propuestos. En una primera aproximación al problema, se usan únicamente monopolos para que el método propuesto se adapte a cualquier algoritmo de simulación mediante aproximaciones en tiempo discreto para señales sinusoidales. A continuación, dicho método se extiende para señales en banda ancha, cuya directividad tiene dependencia con la frecuencia.

Palabras clave: Impedancia de reacción local, métodos en tiempo discreto, modelo de fuentes sonoras, problema no homogéneo de contorno, simulación acústica de salas. 


\section{Resum}

La distribució del camp sonor en una habitació és la conseqüència de les propietats ondulatòries de les fonts acústiques i de la posició, geometria i propietats absorbents de les parets que conformen els límits de l'habitació (condicions de contorn). Encara que hi ha una consolidada teoria acústica ondulatòria, és molt complex, pràcticament impossible, trobar una expressió analítica de la distribució de les variables acústiques en una sala real, en funció de la posició i del temps. Esta situació ha de veure's com un problema no homogeni de contorn, on donada la complexitat de les condicions de contorn i de les fonts sonores, estes fan que el problema siga extremadament complex de resoldre.

La simulació acústica de sales, com és tractada durant esta tesi, comprén l'aproximació algebraica per a resoldre la equació d'onda i el mode de definir les fonts sonores i les condicions de contorn que caracteritzen l'escenari baix anàlisi.

Els mètodes numèrics proporcionen algoritmes precisos per a este propòsit, i entre les diferents possibilitats, l'ús de mètodes en temps discret sorgix com una solució adequada per a resoldre eixes equacions en derivades parcials, particularitzades per a certes condicions. Junt amb el constant creixement de la capacitat de computació actual, creix la conveniència d'estos mètodes per a la simulació acústica de sales. No obstant, fins a ara, hi ha una considerable falta de precisió en la definició d'algunes d'estes condicions: les actuals condicions de contorn dependents de la freqüència no complixen cap model físic, i les fonts directives en els mètodes en temps discret, a penes han sigut tractades.

Esta tesi tracta sobre l'estat de l'art actual de les condicions de contorn i dels models de fonts sonores en els mètodes en temps discret i contribuïx amb certs algoritmes per a millorar la formulació de les condicions de contorn, en el sentit de impedància de reacció local, i els models de les fonts sonores, en termes de la directivitat radiant d'acord amb un patró de radiació determinat. Estos algoritmes han sigut particularitzats per al mètode de les Diferències Finites en el Domini del Temps i per a la Malla de Guies d'Ones Digitals.

Respecte a les condicions de contorn, dos aproximacions diferents han sigut proposades a fi d'aconseguir impedàncies de reacció local: la primera 
consistix a modificar el factor de reflexió d'acord amb l'angle d'arribada d'una onda plana; mentres que l'altra solució proposada, definix les condicions de contorn en termes de la impedància, que és independent del angle d'arribada. Ambdós mètodes han sigut implementats per a les Malles de Guies d'Ones Digitals. A partir dels resultats obtinguts, els avantatges i inconvenients es discutixen al llarg de esta tesi. A més, basat en estos nous mètodes, es proposa una definició de condicions de contorn per al mètode de les diferències finites, de manera semblant als anteriors.

Respecte al nou tema proposat de les fonts directives per a mètodes en temps discret, es proposa uns nous algoritmes. En una primera aproximació al problema, s'usen únicament monopoles perquè el mètode proposat s'adapte a qualsevol algoritme de simulació per mitjà de aproximacions en temps discret per a senyals sinusoïdals. A continuació, el dit mètode s'estén per a senyals en banda ampla, la directivitat del qual té dependència amb la freqüència.

Paraules clau: Impedància de reacció local, mètodes en el temps discret, model de fonts sonores, problema no homogeni de contorn, simulació acústica de sales. 


\section{Abbreviation and Acronyms}

1-D

2-D

3-D

$\mathrm{ABC}$

FEM

CAD

DWG

DWM

FEM

FDTD

FIR

FFT

FTM

IIR

K-DWM

LRI

MLS

ODE

PDE

PML

SI

SSS

TDWM

TLM

ULS

WDF

W-DWM
One dimensional

Two dimensional

Three dimensional

Absorbing Boundary Conditions

Boundary Elements Method

Computer Aided Design

Digital Wave Guide

Digital Waveguide Mesh

Finite Elements Method

Finite-Difference Time-Domain

Finite Impulse Response

Fast Fourier Transform

Functional Transformation Method

Infinite Impulse Response

Kirchhoff Digital Waveguide Mesh

Locally reacting impedance

Maximum-Length Sequence

Ordinary Differential Equation

Partial Differential Equation

Perfect Matched Layer

International System (units)

Space-State Structure

Triangular Digital Waveguide Mesh

Transmission Line Matrix

Upwind Leapfrog Scheme

Wave Digital Filter

Wave Digital Waveguide Mesh 


\section{Notations and Conventions}

\section{Conventions}

The next conventions are used throughout this thesis:

- Time-domain scalar quantities are denoted by lowercase characters, e.g., $a(t)$.

- Frequency-domain scalar quantities are denoted by uppercase characters, e.g., $A(\omega)$.

- Time-domain vector quantities are denoted by boldface lowercase characters, e.g., $\mathbf{a}(t)$.

- Frequency-domain vector quantities are denoted by boldface uppercase characters, e.g., $\mathbf{A}(\omega)$.

- Time-domain matrix quantities are denoted by underlined, boldface lowercase characters, e.g., $\underline{\mathbf{a}}(t)$.

- Frequency-domain matrix quantities are denoted by lowercase characters, e.g., $\underline{\mathbf{A}}(\omega)$.

- Discretized vector or matrix are denoted by tilde characters, e.g., ã and $\underline{\tilde{a}}$

\section{Mathematical operations}
$(\cdot)^{T}$
Vector or matrix transposition
$(\cdot)_{\mathrm{av}}$
Time-averaged
$(\cdot)^{*}$
Vector or matrix conjugated
$(\cdot)^{-1}$
Vector or matrix inverse
$\|\cdot\|$
$\mathrm{L}^{2}$ Norm or vector norm
$\int_{-\infty}^{t} f(\tau) d \tau$
Integration operator w.r.t. $t$
$\mathcal{F}_{t}$
Time Fourier Transform
$\Im\{\cdot\}$
Imaginary component 
$\mathcal{L}$

$\partial / \partial t$

$\Re\{\cdot\}$

$\nabla$

$\nabla^{2}$

$*$

$\angle$
Laplace Transform

Partial derivative w.r.t. $t$

Real component

Nabla operator (gradient)

Laplace operator

Convolution operator

Angular component of a complex number 


\section{List of symbols}

\section{Variables and constants}

$(\cdot)_{i}$

$(\cdot)_{r}$

$\alpha$

A

$a, a_{n}$

$b, b_{n}$

$c$

$c_{n}$

$\underline{\mathbf{C}}(\omega)$

$d$

$D_{s}(\theta, \phi)$

$\Delta t$

$\Delta \theta$

$\Delta x, \Delta y, \Delta z$

$e(\mathbf{r}, t), E(\mathbf{r}, \omega)$

$f$

$\mathbf{i}(t), \mathbf{I}(\omega)$

j

$J_{o}$

$J(\theta, \phi)$

$k$

$\lambda$

$n$

$\hat{\mathbf{n}}$

$\omega$

$p(\mathbf{r}, t), P(\mathbf{r}, \omega)$

$\mathbf{r}=(\|\mathbf{r}\|, \theta, \phi)$

$\mathbf{r}=(x, y, z)$

$\tilde{\mathbf{r}}=(i, j, k)$

$r(\theta, t), R(\theta, \omega)$

$\rho$

$\rho_{0}$

$s(\mathbf{r}, t), S(\mathbf{r}, \omega)$

$S$
Incident component of the variable

Reflected component of the variable

Diagonal direction with respect to

the mesh coordinate system

Absorption coefficient

Incoming wave digital variable, oriented along $\hat{\mathbf{n}}$ direction

Outgoing wave digital variable, oriented along $\hat{\mathbf{n}}$ direction

Speed of sound

Numerical speed of sound

Green's function matrix, w.r.t. angular frequency $\omega$

Thickness

Source directivity

Discrete time step

Discrete angular step

Discrete spatial steps

Sound energy

Linear frequency

Acoustic intensity vector

Complex number, $j^{2}=-1$

Radiation pattern of an omnidirectional source

Radiation pattern of a source

Wavenumber

Wavelength

Discrete time

Normal direction of the wall

Angular frequency

Pressure

Continuous spherical coordinates

Continuous cartesian coordinates

Discrete cartesian coordinates

Reflection factor filter response w.r.t. angle of arrival $\theta$

Density of the medium

Density of the air

Source term

Surface 


$\begin{array}{ll}\sigma & \text { Material's flow resistivity } \\ t & \text { Continuous time } \\ T & \text { Sampling period } \\ T_{\mathrm{amb}} & \text { Ambient temperature } \\ \tau & \text { Time delay } \\ \theta & \text { Plane wave angle of arrival } \\ \theta^{\prime} & \text { Degree of freedom in the reflection factor digital filter } \\ \mathbf{u}(\mathbf{r}, t), \mathbf{U}(\mathbf{r}, \omega) & \text { Particle or fluid velocity } \\ V & \text { Volume } \\ w(\mathbf{r}, t), W(\mathbf{r}, \omega) & \text { Sound energy density } \\ Y & \text { Specific acoustic admittance of a wall } \\ z & \text { Z-transform variable } \\ z^{-1} & \text { Unit delay } \\ Z & \text { Specific acoustic impedance of a wall } \\ Z_{p} & \text { Wave digital port impedance } \\ \zeta & \text { Characteristic acoustic impedance }\end{array}$

\section{Special functions}

$\delta(n) \quad$ Dirac Delta function

$h_{n}^{(1)}(x)$ Spherical Hankel function of first kind of order $n$, w.r.t. argument $x$

$P_{m}^{n}(x) \quad$ Associated Legendre function of order $n$ and degree $m$, w.r.t. argument $x$

$Y_{m}^{n}(x) \quad$ Spherical harmonics of order $n$ and degree $m$, w.r.t. argument $x$ 


\section{Acknowledgments}

Between grief and nothing, I will take grief (The Wild Palms, W. Faulkner, 1939)

Our battered suitcases were piled on the sidewalk again; we had longer ways to go. But no matter, the road is life.

(On the road, J. Kerouac, 1951)

This section, it is without question, is the hardest to write. I should have to summarize my gratitude and appreciation of all people who have influence, support and love me during all these four years, and this is truly hard task since I will never express it as I would really want. But of course, I will try to do my best.

This dissertation is the result of the work of four years which have been really exciting, tough and funny. I feel very lucky to have work with amazing people who have not only influenced this thesis, but also in the rest of my researching/teaching career and they will be an example about what I would like to be one day. Furthermore, during these years not all was long nights in front of a computer: I have meet people who have touch my heart and nowadays, I can call many of them, friends. It is a very pleasant aspect that I have now the opportunity to express my gratitude for all of them.

My first acknowledgement goes to my supervisor Prof. José Javier (J.J.) López. He not only support this thesis from the first moment (despite long distances), but also he teach me that we have to do this work only because is just fun and that is what we like. He has been a great pleasure to work with him, and of course, after this, we will keep working together in the same way. We have shared a lot of beers talking about this moment around the world (New York, Copenhagen, Berlin, Paris,...) and finally, it seems it has arrived.

First steps of this thesis were done during my stay at the University of Erlangen-Nüremberg, in the Chair of Multimedia Communications and Signal Processing. I am very grateful to Prof. Rudolf Rabenstein for trust me and give me that opportunity to work with him: those three months were a fundamental part of this thesis; furthermore, his support during these last years has been really appreciated. I also want to thank Stefan Petrausch 
for his help during that period, for his tolerance with my english skills and for explain me with patience how Wave Digital Filters and Functional Transformation Method work; it was a pleasure to learn from him. I cannot forget to mention my office's colleges: Sascha Spors, Heinz Teutsch and Achim Kuntz.

An important part of my research was done during a year period in the Technical University of Denmark, in Ørsted•DTU, Acoustic Technology Section, thanks to a Marie Curie Fellowship, European Doctorate in Sound and Vibration Studies (EDSVS). That period would not have been possible unless Prof. Finn Jacobsen gave the opportunity to work at his side. It was an incredible experience and I have the chance to learn a lot of things related with this research field. Prof. Jacobsen's contributions to this thesis should be emphasized, and I sincerely hope this was not the last chance to work together.

I want to thanks to the reviewers of this thesis: Prof. Angelo Farina (University of Parma), Prof. Felipe Orduña (National Autonomous University of Mexico) and Prof. Rudolf Rabenstein (University of ErlangenNüremberg) for their valuable comments, which have substantially improved the final version of this thesis. It is especially remarkable the observations of my research coming from Prof. Damian Murphy (University of York) and for its support, and I hope EuRoAPA becomes a reality and the beginning of a fruitful collaboration.

I want to express my gratitude to all the people of my current Department (Telecommunication Engineering) in the University of Jaén. I want to be very grateful to Pedro Reche (the biggest one), for those long mathematical discussions and for his interest in this thesis; and also to those very special colleges whom make my work a little bit more funny and comfortable in Linares: Raquel Viciana, Jose Manuel Pérez, Enrique Muñoz and Fran Cañadas (we have a common scope during next years, but do not worry, we will get it). It is also very valuable the support of Sebastián Garcia, Raúl Mata, Julio Carabias, Juan Carlos Cuevas, Luis Ramón López, Jose Miguel García and Fernando Rivas. I also want to thanks to Nicolás Ruiz and Pedro Vera for give me this opportunity to start my teaching career and let me keep on working in my research field.

I want to thank whom were my professors during 1999-2002, and later they become my friends in University of Alicante, during those hopeless and gloomy three years working as student assistant in DFISTS: Miguel Romá, David Ballester, Juan Manuel López, Tomás Martínez and Jesús 
Selva. They are an example of strength, principles and moral values (keep on fighting!).

I should also thank to my colleges from the Audio Processing and Communication Group, in the Technical University of Valencia: Laura Fuster, Carmen Botella, Teresa Bravo, Miguel Ferrer, Pedro Zuccarello and German Ramos; it was a really short period sharing that office in iTEAM; I enjoyed that working/friendly ambient and every time I come back to visit them, I feel like home. I also want to thank Prof. Alberto Gonzalez his support and for give me that second chance to join GTAC.

This thesis topic (room acoustic simulation) arises from my love to the music: everyone who has seen me working, they have found me always with headphones, listening music. My final aim with this thesis was (and it will be in my future research) to (modestly) contribute to a better music quality perception and new musical experiences through realistic synthesis of room impulse responses. I could live without music (from Young to Hendrix, from Van Morrison to Led Zeppelin), but it would not be the same life.

Since my experience at different universities, I want to thank to all that people whose example teach me what kind of university/research/teaching I do not want to participate with and how this work should not be used for personal ambitious.

To be in Copenhagen it was one of the best times of my life: it was hard, cold and dark, but without any doubt, I would repeat that experience. I want to thanks (and to remember) those great times with Zdenek Havránek, Nick Stefanakis, Marta Papini, Pedro Nunes, Elia Tomás and Manuel Pinelo, and especially, to the ones who were my family during that period, Manuel Quirós and Quka Roldán. I really miss to be there, what we had and what we did during that year. We will always have The Moose.

I feel a deep sense of gratitude to my friends Máximo Cobos and Carlos Gonzaga (future PhDs) for their encourage during these years. A very special thanks to my best friends in the whole world: Majo Blanes, Shukri Bassoumi, Esteban Esteso and Pablo Domenech. We do not see each other as often as we would like, but we will still have a lot of great moments to share.

One of the persons who has a key role in this thesis, without any doubt, is Basilio (Bass) Pueo. Since he was my Bachelor's thesis supervisor in 2002 (he was the responsible of my decision to start my $\mathrm{PhD}$ ), we have shared this path to fulfill one of our longed-for objectives, and it seems we are closer; although and I am afraid, this is not the end and it's too late to stop 
now. Thanks for all, Bass. Without you, this thesis would never exist.

Of course, the support of my family, specially from my mother Reme, has been decisive to do this, despite the distance. The (saddest) memory of my father, Pepe, is the only reason to not enjoy this moment completely. Since he past away, things are not the same and it hurts every moment without him. This is not the same without you, dad.

Finally, the most important person of my life, Caridad (Cari) Guardiola. It was not easy to be far from her and especially, during my danish adventure. She had (and has) an incredible patience with that kind of workaholism which has been characteristic of me during these last years (no more thesis, I promise!). Her dedication, love and persistence in me, has taken the load off my shoulder. I hope we keep sharing those concerts, trips, movies and all those things which form our particular universe (it would take another thesis to express my feelings for you). I still think I do not deserve to be with her, but I am also sure I would be breathless without her. 


\section{Contents}

$\begin{array}{ll}\text { Abstract } & \text { i }\end{array}$

$\begin{array}{lll}\text { Resumen } & \text { iii }\end{array}$

Resum v v

Abbreviation and Acronyms vii

Notations and Conventions $\quad$ ix

Acknowledgments xiii

1 Introduction and scope 1

1.1 Introduction . . . . . . . . . . . . . . . . 1

1.2 Motivation and scope of the thesis . . . . . . . . 2

1.3 Organization of the thesis . . . . . . . . . . . . 4

2 Mathematical and Physical

Foundations in Room Acoustic

Simulation $\quad 7$

2.1 Introduction . . . . . . . . . . . . . . . . 7

2.2 Mathematical foundations . . . . . . . . . . . . 8

2.3 Physical foundations . . . . . . . . . . . . . . . . 11

2.3.1 Euler and conservation of mass equation . . . . . . 12

2.3.2 Solutions to the wave equation . . . . . . . . . . . . 15

2.3.2.1 Plane waves . . . . . . . . . . . . 16

2.3.2.2 Spherical waves . . . . . . . . . . . . . 17

2.3.2.3 Acoustic energy, intensity and source power 19

2.4 Some sound propagation considerations in enclosures . . . . 21

2.4.1 Boundary conditions . . . . . . . . . . . . . 22

2.4.1.1 Wall impedance, reflection factor and absorbing coefficient ........... 22

2.4.1.2 Fundamental conditions for physically representable impedance model . . . . . . . . . 24 
2.4.1.3 The locally reacting impedance problem . . 25

2.4.1.4 Hard-backed layer of porous material impedance . . . . . . . . . . . . . 28

2.4.2 Sound source modeling . . . . . . . . . . . . . . . 31

2.4.2.1 Directive sources . . . . . . . . . . . . 31

2.4.2.2 Some basic directive sources: from monopoles to quadripoles . . . . . . . . . . . 33

2.5 Room acoustics as an inhomogeneous boundary value problem 39

2.6 Discussion .................... . . 40

\section{Room Acoustics}

Simulation Techniques $\quad 43$

3.1 Introduction . . . . . . . . . . . . . . . . . . . . . 43

3.2 Geometrical methods . . . . . . . . . . . . . . . . . . 44

3.2.1 Ray-tracing method . . . . . . . . . . . . . . 45

3.2.2 Image-source method . . . . . . . . . . . . . . . . . . 46

3.2.3 Hybrid and alternative geometrical methods . . . . . 47

3.3 Wave methods in the frequency domain . . . . . . . . . . . 49

3.3.1 Finite Element Method . . . . . . . . . . . . . . . . 49

3.3.2 Boundary Element Method . . . . . . . . . . . . 51

3.4 Wave methods in the time domain . . . . . . . . . . . . 51

3.4.1 Suitability of the discrete-time methods for room acoustic simulation: a survey . . . . . . . . . . . . . 52

3.4.2 Alternative methods in discrete-time modeling . . . 57

3.5 Discussion .................... 59

4 Discrete Time-based Methods

for Room Acoustic Simulation $\quad 61$

4.1 Introduction . . . . . . . . . . . . . . . . 62

4.2 The Finite-Difference Time-Domain (FDTD) method . . . . 63

4.2 .1 Grid Dispersion . . . . . . . . . . . . . . . . . 68

4.2 .2 Stability . . . . . . . . . . . . . 70

4.2.3 The FDTD method in a homogeneous medium . . . 72

4.2.4 The FDTD method in an inhomogeneous medium . 73

4.3 The Digital Waveguide Mesh (DWM) method . . . . . . . 76

4.3.1 Plane waves in the DWM model . . . . . . . . . 80

4.4 Equivalence between the FDTD and DWM methods . . . . 82

4.5 Hybrid models . . . . . . . . . . . . . . . . . . 86

4.6 Sound propagation simulation through discrete-time methods 88 
4.7 Wave Digital Filters . . . . . . . . . . . . . . . 96

4.7.1 A WDF variables approach . . . . . . . . . 96

4.7.2 Wave digital elements . . . . . . . . . . . . . 97

4.7.3 Wave digital element interconnection . . . . . . . . . 99

4.8 Discussion . . . . . . . . . . . . . . . . . . . . . . . . 102

\section{$5 \quad$ Frequency-Dependent Boundary}

Conditions for Discrete-Time Methods 105

5.1 Introduction . . . . . . . . . . . . . . . . . 106

5.2 Boundary conditions in the DWM method . . . . . . . . 107

5.2 .1 Previous approaches . . . . . . . . . . . . 107

5.2.2 Physical interpretation of the use of the reflection factor as a boundary condition . . . . . . . . . . 112

5.2.2.1 Examples . . . . . . . . . . . . . 115

5.2.3 Realization of a LRI model in a DWM based on angle detection . . . . . . . . . . . . . . . 118

5.2.3.1 Angle detection . . . . . . . . . . . . . 119

5.2.3.2 Filter compensation . . . . . . . . . . . . . 124

5.2 .3 .3 Examples . . . . . . . . . . . . . . . 126

5.2.3.4 Limitations of the method . . . . . . . . 127

5.2.4 Realization of a LRI model in a DWM based on mixing modeling strategies . . . . . . . . . . . . . . 129

5.2.4.1 Mixing modeling strategy . . . . . . . . . . 129

5.2.4.2 Impedance model . . . . . . . . . . . . . . 131

5.2.4.3 Model description . . . . . . . . . . . . . 133

5.2.4.4 Analysis of the system . . . . . . . . . . . 138

5.2.4.5 Examples . . . . . . . . . . . . . . . . 141

5.3 Boundary conditions in the FDTD method . . . . . . . . 147

5.3.1 Previous approaches . . . . . . . . . . . . . 147

5.3.2 Proposed method . . . . . . . . . . . . . . . . . . 149

5.3.2.1 Interaction between FDTD and wave variables149

5.3.2.2 Coupling model . . . . . . . . . . . . . 152

5.3.2.3 Examples . . . . . . . . . . . . . . 152

5.4 Experimental evaluation of boundary conditions in a DWM 160

5.4 .1 Measurement set-up . . . . . . . . . . . . . . 161

5.4 .2 Simulation set-up . . . . . . . . . . . . . . 163

5.4.3 Discussion of the results . . . . . . . . . . . 164

5.5 Discussion . . . . . . . . . . . . . . . . 165 
6 Directive Sources

in Discrete-Time Methods $\quad 169$

6.1 Introduction . . . . . . . . . . . . . . . . 170

6.2 Sinusoidal directive sources f . . . . . . . . . . . . 171

6.2 .1 Examples . . . . . . . . . . . . . . . . . 174

6.2.2 Influence of the mesh dispersion . . . . . . . . . . . 179

6.3 Broadband directive sources . . . . . . . . . . . . . . . . 183

6.3.1 Examples . . . . . . . . . . . . . . . . 185

6.4 Discussion . . . . . . . . . . . . . . . . . . . . . 194

7 Conclusions

and Future Research Lines $\quad 195$

7.1 Summary and conclusions . . . . . . . . . . . . . . . . 195

7.2 Contributions of this thesis . . . . . . . . . . . . 197

7.3 Future research lines . . . . . . . . . . . . . . . . 200

A A note on the dispersion

of some discrete-time methods $\quad 203$

B Software implementation 207

B.1 Mesh definition . . . . . . . . . . . . . . . 207

B.1.1 Verifying the mesh modeling . . . . . . . . . . 209

B.1.2 Example . . . . . . . . . . . . . . . . . 211

B.2 Graphical user interface . . . . . . . . . . . . . . . 212

B.3 Parallelization process . . . . . . . . . . . . . 215

B.3.1 Parallel architectures . . . . . . . . . . . . . . . 217

B.3.2 Hardware and software used in the experiments . . . 217

B.4 Computational cost and memory requirements . . . . . . . 218 


\section{Introduction and scope}

\subsection{Introduction}

FROM OUR OWN EXPERIENCE AND WITHOUT ANY SPECIAL TRAINING, one can distinguish and estimate by listenings the volume and the amount of absorption of a room. The result of the particular sound distribution in a room is the consequence of a certain sound wave phenomena appearing due to the geometric distribution and absorbing properties of the walls, ceiling and floor. This makes that each room "sounds" different from the rest. Furthermore, the particular combination of acoustic phenomena gives a distribution of acoustic variables which strongly depends on position and time. When an architect designs a concert hall, theater or lecture room, even with the help of experienced acousticians, the final acoustic result is hard to predict even, unfortunately, impossible [Beranek, 1962].

Although strong mathematical and physical knowledge of the acoustic in rooms exists, it is extremely hard to find analytical expressions of the sound variables distribution, except in very simple and unrealistic scenarios, able to synthesize the complexity of a particular room. For this reason, the use of computers to predict the room acoustic field arises as an important contribution in the design of room acoustics [Schroeder, 1973]. 
The final scopes of these simulations are, mainly, two:

- As a prediction tool, it could allow architects and engineers to determine, with a certain degree of accuracy, the acoustic quality of a room, according to the size and shape of the room, and the materials involved in its construction.

- Given an anechoic sound recording (only direct sound from the source with no contributions from where it was produced), it is possible to modify this sound based on information obtained from the simulations. After hearing this filtered sound, one should have the impression the sound has been played in a particular room [Farina, 1993]; the listener should "perceive" some information of the characteristics of the virtual hall (size, materials, ...). This process, called auralization [Kleiner et al., 1990], can be seen as a convolution process of the original sound with the simulated impulse responses obtained from the virtual room.

Computer room acoustic simulation is an important tool for accurate predicting and then, for improving the quality of built or unbuilt rooms. The computer game and entertainment industries together with training simulators based on virtual reality technologies need, day after day, to find more realistic auditive sensations in order to create a highly immersive environment with spatial attributes [Begault, 1994].

\subsection{Motivation and scope of the thesis}

Although it is essential to be able to reproduce spatial sound information, it is equally important to realistically simulate phenomena occurring during sound propagation. For that reason, a special effort has been made during the last decades in order to develop accurate simulation methods reproducing the characteristics of wave propagation and their interaction with the enclosure, with a reasonably small computational effort.

Among the different solutions appearing in the technical literature, this thesis deals with the computer simulation of virtual acoustic spaces using physics-based models. These methods consist of a numerical approach to the sound wave equation, particularized for the specific boundary conditions 
(walls) and the characteristics of the sources. Mathematically speaking, they could be seen as numerical methods for solving an inhomogeneous boundary value problem.

Going into details, some of these techniques, grouped and called discretetime methods ${ }^{1}$, allow us to approximate the sound pressure distribution in a room, obtaining directly the impulse responses of the entire scenario. They are based on a discretization, not only in space, but also in time, and recursively solve a set of algebraical expressions coming from the original partial differential equations or the potential propagating wave shapes, which describe the sound propagation phenomena. Since the sound field is the result of an approximated solution of the wave equations, the accuracy of the solution can be assured. In fact, all the wave acoustic phenomena such as diffraction, wave superposition, ..., appear in a direct way, with the exception of sound absorption in air.

Although several methods have been developed for this purpose, in room acoustic simulation the most usual techniques are the Finite-Difference Time-Domain (FDTD) method and the Digital Waveguide Mesh (DWM) method. They reproduce the sound field evolution in time (impulse response) for every discretized point of the room. Together with the constant growth of computer power, these methods are increasing their suitability for room acoustic simulation. Many aspects of these methods have been improved, increasing their accuracy and keeping a reasonable computation cost for the simulations.

However, many problems involved in room acoustic simulation by means of discrete-time methods are not solved yet, and the ones this thesis deals with, these are the following:

1. Boundary conditions: most of the walls present in an enclosure are considered reflecting waves in a specular way, under the assumption

\footnotetext{
${ }^{1}$ The concept discrete-time modeling has been widely used in digital sound synthesis [Välimäki et al., 2006], but not really in room acoustic simulation where wave-based methods in the time domain [Savioja, 1999] is the most common denomination. We strongly believe discrete-time methods is an accurate sentence to describe these methods. Furthermore, since physical-based modeling for digital sound synthesis and room acoustic simulation share many advances and methodology (room acoustics is a 3-D problem whereas digital sound synthesis consist of solving, mostly, the same problem but on 1-D or 2-D [Fontana, 2003]) and they progress in the same direction, then, it is fair to think about a common term to describe them.
} 
of a locally reacting impedance model. So far, few contributions to this point have appeared for frequency-dependent absorbing boundary conditions, and a generalized and accurate method capable of dealing with them is still unknown.

2. Sound source modeling: so far, only point or plane sources have been considered in these methods. However, real sources are far from being considered such as those, since they have an irregular pattern of radiation, and very frequently, a frequency dependence.

Both topics are fundamental for a correct and accurate simulation, since variations in these conditions have a considerable influence in the final result, and even in the correct perception of the spatial sound distribution in their use for auralization [Dalenbäck et al., 1993].

After introducing some of the current limitations of discrete-time methods in the previous section, the main scope of this thesis is

To contribute, on the one hand, to boundary condition modeling in the discrete-time methods, which results in allowing one to include locally reacting impedances with a reduced computational cost. On the other hand, to deal with the sound source modeling in these methods, offering algorithms adequate to define radiating sources under a particularized radiation pattern. These algorithms will be validated by comparison with corresponding analytical expressions.

\subsection{Organization of the thesis}

This thesis is organized as follows:

- Chapter 2: This chapter presents a brief summary of the basic mathematical and physical fundamentals involved in sound propagation in enclosures, paying special attention to the boundary conditions and sound source modeling in room acoustics. These notions are necessary for a proper comprehension of this dissertation; however, a reader with some experience in this field, could just overview this chapter.

- Chapter 3: Since the mathematical description of sound distribution in a room is an inhomogeneous boundary value problem, the solution of realistic enclosures must be obtained through techniques that 
simplify those calculations. This chapter discusses various simulation approaches used in virtual acoustics. Finally, after justifying the suitability of the so-called discrete-time methods for room acoustic simulation, a survey of those methods is presented. Furthermore, a brief review of some alternative discrete-time methods is included.

- Chapter 4: This chapter deals with the mathematical description of the most important discrete-time methods: The Finite-Difference Time-Domain method and the Digital Waveguide Mesh method. Differences and similarities are emphasized, arriving at a hybrid model as a convenient solution. Furthermore, a short overview of the Wave Digital Filters (WDFs) is presented, which theory is fundamental for the contributions which have been developed in Chap. 5.

- Chapter 5: In this chapter, the first group of original contributions is presented, which are related to the boundary conditions, particularly, how to perform a locally reacting impedance model for discrete-time methods. First, a review of the previous models is presented, followed by a detailed analysis of the behavior of these algorithms. Since these methods are not able to model boundary conditions with accuracy, some new algorithms are introduced for the DWM, and also for FDTD method.

- Chapter 6: The second group of original contributions is based on the incorporation of directive sources in the discrete-time methods. For given directivity patterns, an algorithm is introduced to perform non-uniform radiating source patterns for sinusoidal sources; also, this algorithm is extended to broadband sources, with variable directivity as a function of frequency.

- Chapter 7: Finally, conclusions obtained in this dissertation are presented, including some guidelines for future research, opened by this thesis.

Additionally, two appendixes supplement the main work, organized as follows:

1. Appendix A: deals with qualitative, not quantitative, aspects of the dispersion of some of the discrete-time methods which appear throughout this thesis. 
2. Appendix B: shows some aspects of design of room acoustics software based on discrete-time methods. Although a front-end software has been considered outside from the scope of this thesis, some thoughts about what should be the first steps in order to design a room acoustic computer-based program are presented. 


\section{Mathematical and Physical Foundations in Room Acoustic Simulation}

The COMPUTER RoOm ACOUSTIC SIMUlation METHODS arise from a set of simplifications concerning to the sound wave propagation theory. Then, it is mandatory in any computer method proposal to present a consistent background of the room acoustic phenomena. This sound wave phenomenology is the starting point in order to develop a solid method for room acoustics.

This chapter concerns the acoustic propagation in unbounded and bounded spaces, where the sound propagation in enclosures is its main aim. In this chapter, some of the fundamental aspects in room acoustic theory are revisited and they form the mathematical and physical basis for the contributions addressed in this thesis.

\subsection{Introduction}

Mathematically, sound propagation can be described as an inhomogeneous boundary value problem [Polyanin and Zaitsev, 2003] through hyperbolic or parabolic differential equations, both being characteristics in wave-like propagation phenomenons. This mathematical description arises from the physical analysis of a time-varying perturbation in a medium. 
Room acoustic is considered as the propagation phenomena where acoustic waves travel through a fluid, mostly air, and interact with the limits of an enclosure, giving a particular sound pressure distribution. Also, sound source properties have a main role in the final room acoustic, and also in its sound perception.

The present chapter deals with the mathematics for sound wave propagation in room acoustics, forming a solid base for understanding the following chapters. It is organized as follows: in the first section, some basic mathematical foundations related to sinusoidal and complex waves are presented. Section 2.3 deals with the equations involved in the sound propagation description in unbounded spaces. In Sec. 2.4, a mathematical and physical description of the sound propagation key concepts in enclosures for this thesis are overviewed, paying especial attention to the boundary conditions (wall properties) and sound source directivity. All the concepts involving the room acoustic modelling concerning this thesis can be expressed in a compact form as an inhomogeneous boundary value problem, which is presented in Sec. 2.5. Finally, the chapter is summarized and discussed.

\subsection{Mathematical foundations}

This section reviews some basic mathematical concepts involved in sound propagation. In order to arrive at a tractable mathematical description of problems related to room acoustics, some a few basic assumptions are introduced here. Let us consider a basic oscillatory function (known also as a sinusoidal wave), $q$, given at a position $\mathbf{r}$ and at instant $t$, which is mathematically defined as

$$
q(\mathbf{r}, t)=Q e^{j(\mathbf{k} \cdot \mathbf{r}-\omega t)},
$$

where $Q$ is the wave amplitude, $\omega$ is the angular frequency and $\mathbf{k}$ is the wavenumber. The angular frequency is related to the linear frequency $f$ through

$$
\omega=2 \pi f
$$

where $f$ is measured in Hertz $(\mathrm{Hz})$ and it indicates the number of oscillations in a second. The temporal period can also be introduced $T$ as the 
inverse of the frequency, indicating how many oscillations occur in a second. Regarding to the wavenumber, its module is defined as

$$
\|\mathbf{k}\|=\frac{\omega}{c},
$$

where $c$ is the speed of sound. Wavenumber is a defined spatial analog of frequency, that is, it is the measurement of the number of repeating units of a propagating wave (the number of times a wave has the same phase) per unit of space. The vectorial form of the wavenumber is usually expressed in spherical coordinates with polar $\theta$ and azimuthal $\phi$ angles as follows

$$
\mathbf{k}=\|\mathbf{k}\| \cos \phi \sin \theta \hat{\mathbf{x}}+\|\mathbf{k}\| \sin \phi \sin \theta \hat{\mathbf{y}}+\|\mathbf{k}\| \cos \theta \hat{\mathbf{z}}
$$

where each wavenumber component is usually defined as

$$
\begin{aligned}
& k_{x}=\|\mathbf{k}\| \cos \phi \sin \theta, \\
& k_{y}=\|\mathbf{k}\| \sin \phi \sin \theta, \\
& k_{z}=\|\mathbf{k}\| \cos \theta,
\end{aligned}
$$

and where the next expression is preserved

$$
\|\mathbf{k}\|^{2}=k_{x}^{2}+k_{y}^{2}+k_{z}^{2}
$$

At the same time, wavelength $\lambda$ is defined as an inverse of the wavenumber through

$$
\lambda=\frac{2 \pi}{\|\mathbf{k}\|},
$$

which denotes the distance, in the wave propagation direction, where equal values of the field quantity occur. From now on, the absolute value of the wavenumber $\|\mathbf{k}\|$, will be simply denoted by $k$, since most texts use this notation.

As will be shown in this thesis, sinusoidal sources are used as mathematical definitions to introduce more complex concepts, since these waves appear in nature (and particularly, in room acoustics) only in very few occasions. However, it is possible to express any periodic function $q(t)$ as a 
linear combination of harmonic waves. This combination is called Fourier series, mathematically expressed as

$$
q(t)=\Re\left[\sum_{n=-\infty}^{+\infty} q_{n} e^{-j \omega_{n} t}\right],
$$

where $\omega_{n}=(2 \pi / T) n$. This means that a periodic function with a period $T=2 \pi / \omega$ is a weighted sum of harmonically related sinusoidal functions.

The coefficients (or Fourier series coefficients) $q_{n}$ are calculated multiplying both parts of Eq. 2.10 by $e^{j \omega_{n} t}$ and integrating over the time segment $T$, obtaining

$$
q_{n}=\left[q(t) e^{j \omega_{n} t}\right]_{\mathrm{av}}=\frac{1}{T} \int_{-\frac{T}{2}+t}^{\frac{T}{2}+t} q(\tau) e^{j \omega_{n} \tau} d \tau .
$$

Until now, only periodic functions have been considered; however, more generalized functions can be represented as a decomposition of harmonic functions. A generic function can be seen as a periodic function, which period is infinite. In that case, basic functions (complex exponential functions) are not harmonically related, but also all frequencies appear. Let us define the continuous Fourier transform as follows

$$
Q(\omega)=\int_{-\infty}^{\infty} q(t) e^{j \omega t} d t=\mathcal{F}_{t}\{q(t)\}
$$

where, for a given angular frequency $\omega_{0}$, the complex value $Q\left(\omega_{0}\right)$ indicates the complex amplitude of the corresponding complex sinusoidal function (Eq. 2.1). The linear operator $\mathcal{F}_{t}\{\cdot\}$ is used as a definition of the Fourier transform. This allows a sinusoidal wave with $\omega=\omega_{0}$ to be defined as

$$
q(\mathbf{r}, t)=Q(\omega) e^{j\left(\mathbf{k} \cdot \mathbf{r}-\omega_{0} t\right)}=Q(\mathbf{r}, \omega) e^{-j \omega_{0} t},
$$

where its corresponding Fourier transform is

$$
\mathcal{F}_{t}\{q(\mathbf{r}, t)\}=Q\left(\mathbf{r}, \omega_{0}\right) .
$$

The Fourier transform is a powerful tool for describing both periodic and non-periodic signals. It specifies the spectral content of a signal. Thus, the Fourier transform provides a frequency-domain description of a signal. 
It is also defined the inverse Fourier Transform, the linear operator which finds the time-domain representation from a frequency domain signal. It is defined as

$$
\mathcal{F}_{t}^{-1}\{Q(\omega)\}=q(t)=\frac{1}{2 \pi} \int_{-\infty}^{\infty} Q(\omega) e^{-j \omega t} d \omega .
$$

The Fourier transform has a set of interesting mathematical properties and some of the most important ones (used during this thesis) are summarized as follows:

- $\mathcal{F}_{t}\{a \cdot q(t)+b f(t)\}=a \cdot Q(\omega)+b \cdot F(\omega)$,

- $\mathcal{F}_{t}\{q(t) \cdot f(t)\}=\frac{1}{2 \pi} Q(\omega) * F(\omega)$,

- $\mathcal{F}_{t}\{q(t) * f(t)\}=Q(\omega) \cdot F(\omega)$,

- $\mathcal{F}_{t}\left\{\frac{\partial q(t)}{\partial t}\right\}=-j \omega Q(\omega)$,

where the operator $*$ indicates the convolution function. Many other properties of the Fourier transform can be found in [Soliman and Srinath, 1997].

\subsection{Physical foundations}

In this section, some of the physical foundations underlying the sound propagation are overviewed. The sound waves are described as compressional oscillatory disturbances that propagate in a fluid [Jacobsen, 2007] or as a result from time-varying perturbation of the dynamic and thermodynamic variables that describe the medium [Pierce, 2007]. In these fluids (or media), two laws appear:

- The time rate of change of the fluid mass is equal to the net mass per unit time entering (minus that leaving) a volume $V$ through a confining surface $S$.

- The mass times acceleration of the center of mass of a fluid particle equals the net apparent force exerted on it by its environment and by external bodies. 
Both laws allow the introduction of two key equations, known as conservation of mass and Euler's equation of motion for a fluid, and they are the basis of the simplest realistic model of sound propagation in fluids. These equations, as will be introduced in Sec. 2.2, relate to the acoustic basic variables: pressure, density and particle (or fluid) velocity.

The pressure $p$ is the force over an area applied on an object in a direction perpendicular to the surface; it is a scalar quantity, and it has SI units of pascals: $1 \mathrm{~Pa}=1 \mathrm{~N} / \mathrm{m}^{2}$.

The particle velocity $\mathbf{u}$, measured in $\mathrm{m} / \mathrm{s}$ as SI units, is the velocity of a particle (real or imaginary) in a medium as it transmits a wave; when applied to a sound wave through a medium of air, particle velocity would be the physical speed of an air molecule as it moves back and forth in the direction the sound wave is travelling as it passes. Since the particle velocity is a vector, it is defined as

$$
\mathbf{u}=u_{x} \hat{\mathbf{x}}+u_{y} \hat{\mathbf{y}}+u_{z} \hat{\mathbf{z}} .
$$

In physics, density $\rho$ is mass $m$ per unit volume $V$, that means the ratio of the amount of matter in an object compared to its volume. The classical model of compressible fluid presumes the existence of some relation between density and pressure:

$$
p=p(\rho) .
$$

In the next subsections, a mathematical model of the sound propagation is introduced, where a set of equations provides a relation between the different acoustic variables, their being the basis of this thesis.

\subsubsection{Euler and conservation of mass equation}

Let us consider for this section a sound propagation free of losses and with no obstacles such as walls (unbounded in all directions). Furthermore, let us assume a medium to be homogeneous and at rest. In accordance to these assumptions, the speed of sound $c$ is constant with reference to time and space. For air, its magnitude is calculated as [Everest, 1994]

$$
c=331.4 \sqrt{1+\frac{T_{\mathrm{amb}}}{273.15}},
$$


and it is approximated as follows [Kuttruff, 4th edition, 2000]:

$$
c=331.4+0.6 T_{\mathrm{amb}},
$$

where $T_{\mathrm{amb}}$ is the ambient temperature in centigrade and $c$ is measured in $\mathrm{m} / \mathrm{s}$. In large halls where there exists some gradient of temperature, this dependence cannot be ignored. However, it is a common practice to assume a constant speed of sound.

As has been previously mentioned, the basic laws for sound propagation are the conservation of mass in a fluid, which is mathematically expressed as

$$
\frac{\partial \rho(\mathbf{r}, t)}{\partial t}+\nabla \cdot(\rho(\mathbf{r}, t) \mathbf{u}(\mathbf{r}, t))=0,
$$

and the Euler's equation of motion in a fluid

$$
\rho(\mathbf{r}, t)\left(\frac{\partial \mathbf{u}(\mathbf{r}, t)}{\partial t}+\mathbf{u}(\mathbf{r}, t) \cdot(\nabla \cdot \mathbf{u}(\mathbf{r}, t))\right)=-\nabla p(\mathbf{r}, t) .
$$

Although these basic acoustic equations are clearly nonlinear, acoustic disturbances can usually be regarded as small-amplitude perturbations to an ambient state and negligible body forces such as gravity, are not considered. This particularity of sound propagation could be applied to the room acoustics and it allows the reduction of governing equations by a first order approximation in the expansion of nonlinear equations.

Let us define the ambient-field or steady-state variables $p_{0}, \rho_{0}$ and $\mathbf{u}_{0}$ in a homogeneous and quiescent medium. Since the medium is homogeneous, the ambient quantities are independent of the position; and quiescent means these variables are independent of time and $\mathbf{u}_{0}=0$. These variables are the solution to the Eqs. 2.20 and 2.21, but when a disturbance modifies the steady-state, one has

$$
p(\mathbf{r}, t)=p_{0}+p^{\prime}(\mathbf{r}, t) \quad \rho(\mathbf{r}, t)=\rho_{0}+\rho^{\prime}(\mathbf{r}, t) .
$$

Substituting Eqs. 2.22 into Eqs. 2.17, 2.20 and 2.21, giving the following system equations 


$$
\begin{array}{r}
\frac{\partial}{\partial t}\left(\rho_{0}+\rho^{\prime}(\mathbf{r}, t)\right)=-\nabla \cdot\left[\left(\rho_{0}+\rho^{\prime}(\mathbf{r}, t)\right) \mathbf{u}^{\prime}(\mathbf{r}, t)\right], \\
\left(\rho_{0}+\rho^{\prime}(\mathbf{r}, t)\right)\left(\frac{\partial}{\partial t}+\mathbf{u}^{\prime}(\mathbf{r}, t) \cdot \nabla\right) \mathbf{u}(\mathbf{r}, t)^{\prime}=-\nabla\left(p_{0}+p^{\prime}(\mathbf{r}, t)\right), \\
p_{0}+p^{\prime}(\mathbf{r}, t)=p\left(\rho_{0}+\rho^{\prime}(\mathbf{r}, t)\right) .
\end{array}
$$

The terms in Eqs. 2.23 and 2.24 can be grouped into zero-terms (all are identically zero), first-order (just one prime variable), second-order (two prime variables), .. . [Pierce, 1994]. Equation 2.25 can be expanded through a Taylor serie in $\rho^{\prime}$ as

$$
p^{\prime}(\mathbf{r}, t)=\left(\frac{\partial p(\mathbf{r}, t)}{\partial \rho(\mathbf{r}, t)}\right)_{0} \rho^{\prime}(\mathbf{r}, t)+\frac{1}{2}\left(\frac{\partial^{2} p(\mathbf{r}, t)}{\partial \rho^{2}(\mathbf{r}, t)}\right)_{0}\left(\rho^{\prime}(\mathbf{r}, t)\right)^{2}+\ldots,
$$

where the indicated derivatives are evaluated at constant entropy (expressed through subindex 0 ) and with density subsequently set to $\rho_{0}$.

The linear approximation (or so-called acoustic approximation) neglects second- and higher-order terms, giving

$$
\begin{array}{r}
\frac{\partial \rho^{\prime}(\mathbf{r}, t)}{\partial t}+\rho_{0} \nabla \cdot \mathbf{u}^{\prime}(\mathbf{r}, t)=0 \\
\rho_{0} \frac{\partial \mathbf{u}^{\prime}(\mathbf{r}, t)}{\partial t}+\nabla p^{\prime}(\mathbf{r}, t)=0, \\
p^{\prime}(\mathbf{r}, t)=c^{2} \rho^{\prime}(\mathbf{r}, t) \quad c^{2}=\left(\frac{\partial p}{\partial \rho}\right)_{0},
\end{array}
$$

where, based on thermodynamic consideration, requires $c^{2}$ always to be positive [Châtelier, 1888].

These equations are arranged in a set of two equations where, and from now on, the primes are removed:

$$
\begin{aligned}
\frac{\partial p(\mathbf{r}, t)}{\partial t}+\rho_{0} c^{2} \nabla \cdot \mathbf{u}(\mathbf{r}, t) & =0 \\
\rho_{0} \frac{\partial \mathbf{u}(\mathbf{r}, t)}{\partial t}+\nabla p(\mathbf{r}, t) & =0 .
\end{aligned}
$$


There exist some applications where the particle velocity variable is not computed, such as room acoustics. For that reason, it is convenient to introduce the wave equation, consisting of a unique equation with only a pressure variable dependence. From the linear acoustic equations, the linear operator $\frac{1}{\rho_{0}} \nabla \cdot\left(\int_{-\infty}^{t}(\cdot) d \tau\right)$ is applied to Eq. 2.31 and this result is substituted in Eq. 2.30. This sequence of steps yields

$$
\nabla^{2} p(\mathbf{r}, t)-\frac{1}{c^{2}} \frac{\partial^{2} p(\mathbf{r}, t)}{\partial t^{2}}=0
$$

where the operator $\nabla^{2}$ is the Laplacian sum of the second derivatives with respect to the three cartesian coordinates.

When the wave equation includes an independent term which purpose is to model a certain source $s(\mathbf{r}, t)$, Eq. 2.32 becomes the inhomogeneous wave equation $^{1}$ :

$$
\nabla^{2} p(\mathbf{r}, t)-\frac{1}{c^{2}} \frac{\partial^{2} p(\mathbf{r}, t)}{\partial t^{2}}=s(\mathbf{r}, t)
$$

In a case with sinusoidal sound propagation, the wave equation becomes the Helmoltz equation via Fourier transform and Eq. 2.3

$$
\nabla^{2} P(\mathbf{r}, \omega)+k^{2} P(\mathbf{r}, \omega)=S(\mathbf{r}, \omega)
$$

\subsubsection{Solutions to the wave equation}

Since the sound propagation is modelled as a wave equation, there exists a set of basic solutions to these equations (waves), which are interpreted as travelling disturbances through a medium. In this section two simple but basic solutions are presented: plane and spherical waves. Although these solutions are far from real waves, they give the basis to understand the realworld propagation phenomenon of acoustic propagation, since they could be seen as a linear combination of these basic wave shapes.

\footnotetext{
${ }^{1}$ The Euler and conservation of mass equation can also be defined as inhomogeneous PDEs [Ziomek, 1995]; however, this thesis only will concern about the wave equation source terms.
} 


\subsubsection{Plane waves}

One of these basic solutions is the plane wave, which plays a key point in many applications, as it will be seen later, in this thesis. The plane wave is defined when all the acoustic variables vary with time and with some cartesian coordinate $\|\mathbf{r}\|=s$, but are independent of position along planes normal to the $s$ direction, $p=p(s, t)$. Because $\nabla p$ has only $s$ component, the fluid acceleration $\partial \mathbf{u} / \partial t$ must be in the $\pm s$ direction and if the initial particle velocity has no initial components in the transverse direction to $s$, these components will remain zero. If one writes $\mathbf{u}(\mathbf{r}, t)=u(s, t) \hat{\mathbf{n}}_{s}$, where $\hat{\mathbf{n}}_{s}$ is the unit direction of increasing distance $s$ and in accordance to these simplifications, the next equations yield

$$
\begin{aligned}
\frac{\partial p(s, t)}{\partial t}+\rho_{0} c^{2} \frac{u(s, t)}{\partial s} & =0 \\
\rho_{0} \frac{\partial u(s, t)}{\partial t} & =-\frac{\partial p(s, t)}{\partial s},
\end{aligned}
$$

whereas the wave equation reduces to its $1-\mathrm{D}$ form

$$
\frac{\partial^{2} p(s, t)}{\partial s^{2}}-\frac{1}{c^{2}} \frac{\partial^{2} p(s, t)}{\partial t^{2}}=0,
$$

or equivalently

$$
\left(\frac{\partial}{\partial s}-\frac{1}{c} \frac{\partial}{\partial t}\right)\left(\frac{\partial}{\partial s}+\frac{1}{c} \frac{\partial}{\partial t}\right) p(s, t)=0 .
$$

The general solution to the latter equation is a sum of a function of $\xi=t-s / c$ and of a function of $\eta=t+s / c$, that is,

$$
p(s, t)=f(t-s / c)+g(t+s / c),
$$

where the function $f$ and $g$ are arbitrary [Everest, 1994]. This solution to the wave equation is known as the D'Alembert solution.

In order to obtain the relation between the solution for pressure and particle velocity, Eqs. 2.35-2.36 are used, giving

$$
\rho_{0} c\left(\frac{\partial}{\partial t} \pm c \frac{\partial}{\partial s}\right) u(s, t)=\mp\left(\frac{\partial}{\partial t} \pm c \frac{\partial}{\partial s}\right) p(s, t) .
$$


The solution to this equation is

$$
u(s, t)=\left(\rho_{0} c\right)^{-1}[f(t-s / c)-g(t+s / c)],
$$

where the functions $f$ and $g$ are the same as Eq. 2.39.

The wave interpretation of the solution follows since $f(t-s / c)$ and $g(t-s / c)$ describe waves moving in the $+s$ and $-s$ directions, respectively, with speed $c$. If $f(t-s / c)$ is plotted versus $s$ for two fixed successive values of $t$, both wave shapes are identical but separated by distance $c\left(t_{2}-t_{1}\right)$ to the right (see Fig. 2.1 for details).

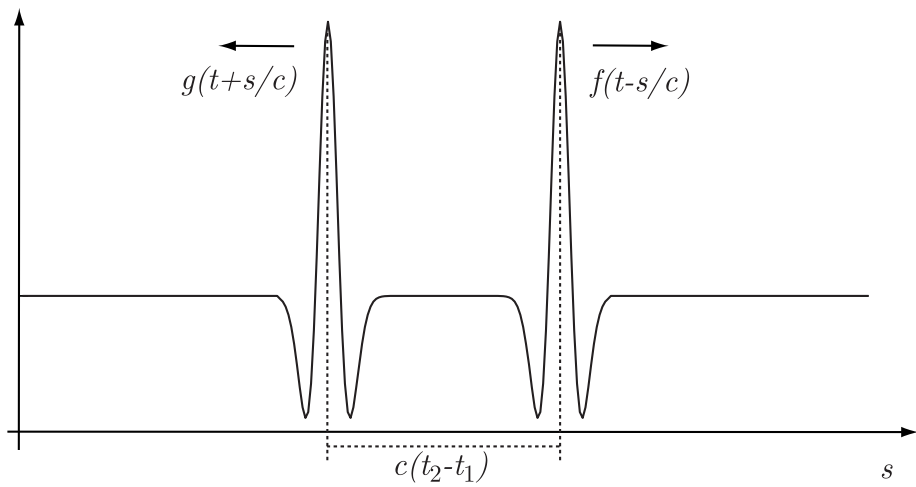

Figure 2.1. Physical interpretation of a 1-D traveling wave based on D'Alembert solution.

In a vectorial form, in a plane wave propagated in a direction $\hat{\mathbf{n}}$, the particle velocity is expressed as follows

$$
\mathbf{u}(\mathbf{r}, t)=\frac{p(\mathbf{r}, t)}{\rho_{0} c} \hat{\mathbf{n}} .
$$

\subsubsection{Spherical waves}

In addition to plane waves, another idealization of the wave propagation corresponds to a symmetric wave spreading in a unbounded fluid medium. The source is considered to be a sphere with radius $a$ centered at the origin.

Since a spherical wave varies symmetrically according to $\|\mathbf{r}\|$, which is the radius distance from the origin, a simple way to derive the wave shape 
of a spherical wave consists on expressing the wave equation into spherical coordinates $(\|\mathbf{r}\|, \theta, \phi)$. After some modifications [Pierce, 1994], the wave equation in spherical coordinates is

$$
\frac{1}{\|\mathbf{r}\|} \frac{\partial^{2}(\|\mathbf{r}\| p(\|\mathbf{r}\|))}{\partial\|\mathbf{r}\|^{2}}-\frac{1}{c^{2}} \frac{\partial^{2} p(\|\mathbf{r}\|)}{\partial t^{2}}=0
$$

where the pressure $p(\|\mathbf{r}\|, t)$ has no dependence on $\theta$ or $\phi$. In a similar fashion to the plane wave, the wave solution to this equation is deduced as

$$
p(\|\mathbf{r}\|, t)=\|\mathbf{r}\|^{-1} f(t-\|\mathbf{r}\| / c)+\|\mathbf{r}\|^{-1} g(t+\|\mathbf{r}\| / c),
$$

where $f$ and $g$ are a priori arbitrary functions.

Outside of the region where the initial conditions are confined, if there are not sources except the one situated at the origin, waves only move in the $+\|\mathbf{r}\|$ direction and thus, $g(t+\|\mathbf{r}\| / c)=0$ (see Fig 2.2).

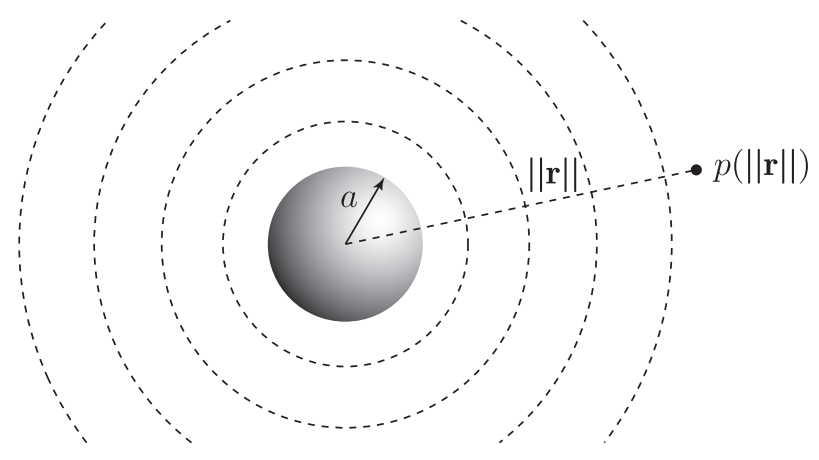

Figure 2.2. Vibrating spherical source with radius a radiating in a free field).

The particle velocity in a spherical wave is not directly obtained as in the plane wave case, since particle velocity and pressure are not proportional. After using some concepts which are far from this thesis (see [Pierce, 1994]), the particle velocity is defined as

$$
\mathbf{u}(\|\mathbf{r}\|, t) \cdot \hat{\mathbf{n}}_{r}=\frac{p(\|\mathbf{r}\|, t)}{\rho_{0} c}+\frac{F(t-\|\mathbf{r}\| / c)}{\rho_{0}\|\mathbf{r}\|^{2}}
$$


where $\hat{\mathbf{n}}_{r}$ indicates the unitary vector in the radius direction and $F(t)=$ $\int_{-\infty}^{t} f(\tau) d \tau$.

An interesting conclusion of Eq. 2.45 arises because of the peak values in time of the second term decreases with distance as $1 /\|\mathbf{r}\|^{2}$ while the first term decreases as $1 /\|\mathbf{r}\|$. This means for a large $\|\mathbf{r}\|$, the second term may be considered negligible compared with the first; then, the asymptotic relation between pressure and particle velocity gives the same relation as in plane waves. For waves of constant frequency, this relation is true when $\|\mathbf{r}\|$ is much larger than its associate wavelength $(\simeq 10 \lambda)$.

\subsubsection{Acoustic energy, intensity and source power}

Since pressure and particle velocity define an acoustic field, it implies moving fluid elements have kinetic energy, and changes in the pressure imply potential energy. Thus, there is a flow of energy involved in the phenomenon of sound; sources of sound emit sound power, and sound waves carry energy [Jacobsen, 2007].

The linear acoustic equations have a corollary related to the energy conservation for an acoustic field [Pierce, 1994], similar to Poynting's theorem in electromagnetism [Balanis, 1989].

To derive this energy conservation, the linear version of the Euler equation has to be taken (Eq. 2.31) and the dot product of $\mathbf{u}$ applied

$$
\begin{aligned}
\mathbf{u}(\mathbf{r}, t) \cdot\left(\rho_{0} \frac{\partial \mathbf{u}(\mathbf{r}, t)}{\partial t}\right) & =-\mathbf{u}(\mathbf{r}, t) \cdot \nabla p(\mathbf{r}, t) \\
& =-\nabla \cdot(\mathbf{u}(\mathbf{r}, t) p(\mathbf{r}, t))+p(\mathbf{r}, t) \nabla \cdot \mathbf{u}(\mathbf{r}, t) \\
& =-\nabla \cdot(p(\mathbf{r}, t) \mathbf{u}(\mathbf{r}, t))-\frac{p(\mathbf{r}, t)}{\rho_{0} c^{2}} \frac{\partial p(\mathbf{r}, t)}{\partial t}
\end{aligned}
$$

Through Eq. 2.31 and Eq. 2.30, Eq. 2.47 it can be reexpressed as [Jacobsen, 2006]

$$
\frac{\partial w(\mathbf{r}, t)}{\partial t}+\nabla \cdot \mathbf{i}(\mathbf{r}, t)=0
$$

where 


$$
\begin{aligned}
w(\mathbf{r}, t) & =\frac{1}{2} \rho_{0}\|\mathbf{u}(\mathbf{r}, t)\|^{2}+\frac{1}{2} \frac{p(\mathbf{r}, t)^{2}}{\rho_{0} c^{2}} \\
\mathbf{i}(\mathbf{r}, t) & =\mathbf{u}(\mathbf{r}, t) p(\mathbf{r}, t) .
\end{aligned}
$$

The variables $w(\mathbf{r}, t)$ and $\mathbf{i}(\mathbf{r}, t)$ are defined as the total instantaneous energy density and the instantaneous sound intensity, respectively. The terms of the total instantaneous energy density correspond with the kinetic energy $\left(\rho_{0}\|\mathbf{u}(\mathbf{r}, t)\|^{2} / 2\right)$ and the potential energy $\left(p^{2}(\mathbf{r}, t) /\left(2 \rho_{0} c^{2}\right)\right)$.

Equation 2.47 is the conservation of sound energy, which expresses the simple fact that the rate of decrease of the sound energy density at a given position in a sound field $(-\partial w(\mathbf{r}, t) / \partial t)$ is equal to the rate of the flow of sound energy diverging away from the point $(\nabla \cdot \mathbf{i}(\mathbf{r}, t))$.

It could be easier to see this fact through the global version, which is obtained through Gauss's theorem, according to which the net outflow of sound energy integrated over a given volume $V$ equals the total net outflow of sound energy through the surface of the volume $S$

$$
\int_{V} \nabla \cdot \mathbf{i}(\mathbf{r}, t) d V=\int_{S} \mathbf{i}(\mathbf{r}, t) \cdot d S=\frac{\partial}{\partial t}\left(\int_{V} w(\mathbf{r}, t) d V\right)=-\frac{\partial e(\mathbf{r}, t)}{\partial t}
$$

which shows that the total net outflow of sound energy through the surface equals the (negative) rate of change of the total sound energy within the surface, $e(\mathbf{r}, t)$. In other words, the rate of change of the sound energy within a closed surface is identical with the surface integral of the normal component of the instantaneous sound intensity, $\mathbf{i}(\mathbf{r}, t)$.

The intensity vector in a plane wave is reduced, according to Eq. 2.42, as follows

$$
\mathbf{i}(\mathbf{r}, t)=\frac{p^{2}(\mathbf{r}, t)}{\rho_{0} c} \hat{\mathbf{n}}=c w(\mathbf{r}, t) \hat{\mathbf{n}},
$$

where the kinetic and potential energies are therefore equal for plane waves.

In the spherical waves case, the intensity vector, according to Eq. 2.45, becomes 


$$
\mathbf{i}(\mathbf{r}, t)=\left[\frac{p^{2}(\mathbf{r}, t)}{\rho_{0} c}+\frac{\partial}{\partial t}\left(\frac{F^{2}(t-r / c)}{2 \rho_{0} r^{3}}\right)\right] \hat{\mathbf{n}} .
$$

For a constant frequency $\omega$ spherical wave $\left(p, \mathbf{u} \propto e^{-j \omega t}\right)$, it is demonstrated [Pierce, 1994] how in the limit $\omega r / c \ll 1$, the energy predominant is kinetic; but in the limit $\omega r / c \gg 1$, the potential and kinetic energy density are the same.

\subsection{Some sound propagation considerations in en- closures}

Up till now, free field conditions have been assumed since an unbounded space is considered and sources are supposed as idealized. However, a room or enclosure is defined as a bounded space, where the iterative reflections of the propagated waves in different walls form the total sound field and real-world sound sources are far from being an idealized plane or spherical wave since their power is not spread evenly. Then, the total sound field in an enclosure is the result of a set of wave phenomena such as reflection, absorption, diffraction,... particularized on the room properties under analysis.

From a mathematical point of view, the total sound field is obtained solving the inhomogeneous wave equation under the specific boundary conditions. These concepts are the basis of the so-called wave theory of room acoustics.

It is not within the scope of this thesis to deal with a detailed overview nor wave theory, nor subjective and objective aspects in room acoustics (this can be consulted in [Kuttruff, 4th edition, 2000]). However, some basis of the sound propagation in enclosures used throughout this thesis are presented. These considerations are, firstly, an overview of the boundary conditions definition, focusing on the absorption and reflection in walls. On the other hand, sound sources are analyzed under the concept of source directivity. 


\subsubsection{Boundary conditions}

In this section, the wall influence in an enclosure is analyzed and modeled. When a wave strikes a wall, part of its energy is reflected. However, these reflections can be categorized in two possibilities: specular and diffuse. In real life, both phenomena occur in each reflection, with different proportions, depending on the wall shape.

Smooth walls produce specular reflections, where the reflected wave keeps the same angle of incidence. Irregularly shaped acoustic boundaries result in diffuse reflections, causing a redistribution of the sound energy across a range of angles upon reflection.

In this thesis, only specular reflections are considered, introducing the locally reacting impedance concept (see Sec. 2.4.1.3), which simplifies the wall properties definition.

\subsubsection{Wall impedance, reflection factor and absorbing coefficient}

The normal surface specific acoustic impedance $Z=Z(\omega)$, or the specific boundary impedance for short, is defined for a given plane wave, as the ratio of complex amplitude and the normal component of the associated particle velocity. Let us consider a plane surface with a specific boundary impedance. This impedance presents a particular response depending on the shape of the incident wave. This is because the normal component of the particle velocity at any point of the surface is influenced not only by the local pressure, but also by the surrounding points. This means that it is not possible to specify a unique boundary impedance, independent of the amplitude and phase distribution of the incident wave over the surface [Fahy, 2001].

However, there are cases where material is able to dissipate energy efficiently, i.e. porous sound absorbing materials [Beranek, 1940], or where only reflection phenomena exists, i.e. ground surfaces [Embleton et al., 1976]. In these scenarios it is reasonable to assume that the particle velocity generated by incident sound at any point is linearly related only to the local sound pressure, and then, independent of the shape of the incident sound field. Therefore, a material surface may be characterized in terms of a unique specific boundary impedance. Fortunately, this impedance can be considered in many practical cases, i.e. room acoustics [Kuttruff, 4th edition, 2000] and mathematically described as 


$$
Z(\omega)=\frac{P(\omega)}{\mathbf{U}(\omega) \cdot \hat{\mathbf{n}}}
$$

where $\hat{\mathbf{n}}$ is the perpendicular direction to the impedance surface, and $P(\omega)$ and $\mathbf{U}(\omega)$ corresponds with the amplitude of a sinusoidal plane wave with angular frequency $\omega$. In the particular case of a plane wave traveling in the air, the specific acoustic impedance is $Z_{\text {air }}=\rho_{0} c$.

Frequently, the wall impedance is divided by the specific acoustic impedance of the air, giving the characteristic acoustic impedance, defined as

$$
\zeta(\omega)=\frac{Z(\omega)}{\rho_{0} c} .
$$

The reciprocal of the specific acoustic impedance is known as specific acoustic admittance, $Y(\omega)$ whereas the characteristic acoustic admittance is the reciprocal from the characteristic acoustic impedance.

The impedance definition (Eq. 2.53) can be inserted into the wave equation through the Euler equation (Eq. 2.31) in the wave domain and obtain

$$
Z(\omega) \frac{\partial P(\mathbf{r}, \omega)}{\partial \hat{\mathbf{n}}}+j \omega \rho_{0} P(\mathbf{r}, \omega)=0,
$$

where $\partial /(\partial \hat{\mathbf{n}})$ denotes partial differentiation in the direction of the outward normal to the wall. It also can be defined in terms of the specific impedance as

$$
\zeta(\omega) \frac{\partial P(\mathbf{r}, \omega)}{\partial \hat{\mathbf{n}}}+j k P(\mathbf{r}, \omega)=0 .
$$

When the plane wave strikes on a surface, the amplitude and phase of the reflected wave changes. This proportion between the reflected and incident wave is characterized by a complex reflection factor

$$
R=\|R\| e^{j \angle R} .
$$

As will be shown in Sec. 2.4.1.3, the reflection factor absolute value $\|R\|$ and phase $\angle R$ is dependent on the frequency and angle of arrival. 
Finally, the sound absorption is defined: according to Eq. 2.51, the intensity of a plane wave is proportional to the square of the pressure amplitude. Therefore, the intensity of the reflected wave is smaller by a factor of $\|R\|^{2}$ than that of the incident wave and the fraction $1-\|R\|^{2}$ of the incident energy is lost during reflection. This quantity is called the absorption coefficient $A$ of the walls

$$
A=1-\|R\|^{2} .
$$

\subsubsection{Fundamental conditions for physically representable impedance model}

Equation 2.53 represents an impedance without mean flow. In the time domain, it could be described as

$$
p(\mathbf{r}, t)=\frac{1}{2 \pi} \int_{-\infty}^{+\infty} z(t-\tau) \mathbf{u}(\mathbf{r}, \tau) \cdot \hat{\mathbf{n}} d \tau,
$$

where $z(t)$ denotes the time domain impedance.

If the impedance is expressed in a rational form $Z(\omega)=A(\omega) / B(\omega)$ it is possible to obtain a convolution on both sides

$$
\int_{-\infty}^{+\infty} a(t-\tau) p(\mathbf{r}, \tau) d \tau=\int_{-\infty}^{+\infty} b(t-\tau) \mathbf{u}(\mathbf{r}, \tau) \cdot \hat{\mathbf{n}} d \tau
$$

From those expressions, let us define some conditions in order to define a physical realizable impedance model [Rienstra, 1998]:

- Since $p(\mathbf{r}, \tau)$ cannot depend on $\mathbf{u}(\mathbf{r}, t) \cdot \hat{\mathbf{n}}$ of the future, it must be defined $z(t-\tau)=0$ for $t>\tau$ and $Z(\omega)$ has to satisfy the causality condition.

- Since $\mathbf{u}(\mathbf{r}, t) \cdot \hat{\mathbf{n}}$ cannot depend on $p(\mathbf{r}, \tau)$ of the future, the admittance $Z(\omega)^{-1}$ must be causal.

- Since $p(\mathbf{r}, \tau)$ and $\mathbf{u}(\mathbf{r}, t) \cdot \hat{\mathbf{n}}$ are real, $z(t)$ has to be real too, and so $Z(\omega)$ has to satisfy the reality condition

$$
Z^{*}(\omega)=Z(-\omega)
$$


In other words, the real part $\Re(Z)$ (resistance) is even and the imaginary part $\Im(Z)$ (reactance) is odd.

- Usually, the impedance wall is a passive wall that absorbs energy at any frequency, i.e. the acoustic intensity into the wall is positive (see Sec. 2.3.2.3). As a result, the resistance $\Re(Z)$ has to be positive. In other words, the impedance satisfies the condition of passivity

$$
\Re(Z(\omega)) \geq 0 \quad \forall \omega \in \mathbb{R} .
$$

It should be noted that violation of the reality condition produces physically impossible results; violation of causality produces instabilities due to "decay" in reversed time; violating the passivity condition implies a wall that produces instead of absorbs energy which will also lead to an instability.

\subsubsection{The locally reacting impedance problem}

In this subsection, the oblique incidence problem is formulated, giving the definition of a locally reacting impedance. Let us consider an infinitely extended impedance surface of uniform impedance, shown in Fig. 2.3(a). On the basis of a locally reacting impedance, it is possible to obtain a general expression for the reflection factor in terms of the impedance and the angle of the sinusoidal plane wave incidence. Let us define an incident plane wave with amplitude $P_{\mathrm{i}}(\omega)$ and angular frequency $\omega$, propagating in a direction $(\cos \theta, \sin \theta)$ where $\theta$ is the angle with the $x$-axis and approaching from $x<0$ and impedance wall $Z=P(\omega) / U_{x}(\omega)$ at $x=0$ :

$$
p_{\mathrm{i}}(\mathbf{r}, t)=P_{\mathrm{i}}(\omega) e^{-j(k x \cos \theta+k y \sin \theta)} e^{j \omega t},
$$

where $k$ is the wavenumber (see Fig. 2.3b) for details).

The reflected wave, with amplitude $p_{\mathrm{r}}$, is propagating in the direction $(-\cos \theta, \sin \theta)$ :

$$
p_{\mathrm{r}}(\mathbf{r}, t)=P_{\mathrm{r}}(\omega) e^{-j(-k x \cos \theta+k y \sin \theta)} e^{j \omega t} .
$$

Both amplitudes are related to the reflection factor $R$

$$
R=P_{\mathrm{r}}(\omega) / P_{\mathrm{i}}(\omega)
$$




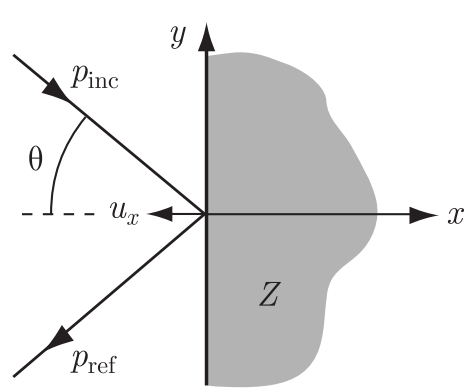

(a)

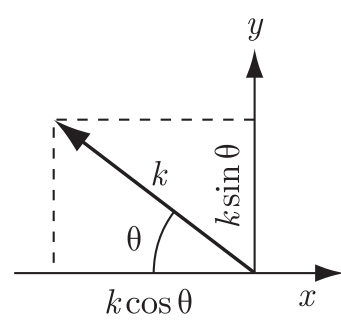

(b)

Figure 2.3. (a) Incidence of a plane wave on an infinite plane surface of uniform impedance. (b) Wavenumber vector components of the incidence wave.

Thus, the total pressure field $p$ is the sum of both components,

$$
\begin{aligned}
p(\mathbf{r}, t) & =\left(P_{\mathrm{i}}(\omega) e^{-j k x \cos \theta}+P_{\mathrm{r}}(\omega) e^{j k x \cos \theta}\right) e^{-j(k y \sin \theta-\omega t)} \\
& =P_{\mathrm{i}}(\omega)\left(e^{-j k x \cos \theta}+R e^{j k x \cos \theta}\right) e^{-j(k y \sin \theta-\omega t)}
\end{aligned}
$$

Using the linearized momentum conservation law $\rho\left(\partial u_{x} / \partial t\right)=-\partial p / \partial x$, the normal component of the particle velocity $u_{x}$ can be derived

$$
u_{x}(\mathbf{r}, t)=\frac{\cos \theta}{\rho c} P_{\mathrm{i}}(\omega)\left(e^{-j k x \cos \theta}-R e^{j k x \cos \theta}\right) e^{-j(k y \sin \theta-\omega t)} .
$$

The latter definition permits the definition of the impedance at the point $x=0$ :

$$
Z=\frac{\rho c}{\cos \theta} \frac{1+R}{1-R}
$$

and the reflection factor

$$
R=\frac{Z \cos \theta-\rho c}{Z \cos \theta+\rho c} .
$$


An impedance with a local reaction assumption or locally reacting impedance (LRI), means that the impedance does not depend on the plane wave angle arrival, e. g. $Z=Z(\omega)$, but the reflection factor does depend on this angle, e. g. $R=R(\theta, \omega)$ :

$$
R(\theta, \omega)=\frac{Z(\omega) \cos \theta-\rho c}{Z(\omega) \cos \theta+\rho c}
$$

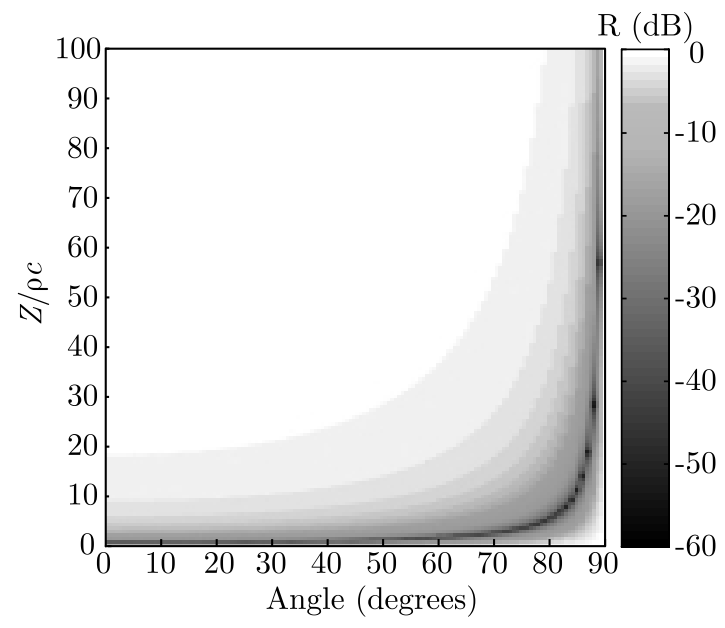

Figure 2.4. Representation of the reflection factor as a function of the impedance and the angle of incidence.

At this point, Eq. 2.70 is graphically represented in Fig. 2.4 in order to better understand its behaviour which will be important later in the proposed model. The reflection factor has been expressed using a gray scale in $\mathrm{dB}$ as a function of the impedance and the angle of arrival. The impedance is represented dimensionless, determined by the ratio of the specific impedance to the characteristic impedance of the medium $\rho c$. In the figure, the considered angle of arrival varies between 0 and $\pi / 2$, whereas the impedance is situated between 0 and 100. It can be observed that when the impedance has low values (high absorption), there are significant differences when the angle of arrival varies. However, if the impedance value increases (low absorption), the dependence on the angle of arrival decreases, especially for low angles of arrival where the reflection factor is almost constant 
at $0 \mathrm{~dB}$. In this case, the highest differences appear when the angle tends to be parallel to the surface. When impedance tends to infinity, the reflection factor will be $0 \mathrm{~dB}$ for all the angles of incidence.

\subsubsection{Hard-backed layer of porous material impedance}

An interesting case of the LRI model is the hard-backed layer of porous material. This consists of a semi-infinite rigid porous layer developed on the basis of semi-empirical considerations. It involves many measurements on fibrous materials that have been used widely for modeling the impedance of outdoor ground surfaces.

The mathematical expression given for a layer with a thickness $d$ [Kuttruff, 4th edition, 2000] is

$$
Z(\omega)=-j Z_{p}(\omega) \cot \left(k_{p}(\omega) d\right),
$$

where the material is modeled using Delany and Bazley's one-parameter model [Delany and Bazley, 1970], that is, the characteristic impedance and wavenumber of the material are given by

$$
\begin{aligned}
Z_{p}(\omega) & =\rho_{0} c\left(1+0.057 X(\omega)^{-0.754}-j 0.087 X(\omega)^{-0.732}\right), \\
k_{p}(\omega) & =\rho_{0} c\left(1+0.098 X(\omega)^{-0.7}-j 0.189 X(\omega)^{-0.595}\right),
\end{aligned}
$$

where $X(\omega)=\rho_{0} \omega /(2 \pi \sigma)$ and $\sigma$ is the material's flow resistivity.

A $0.1 \mathrm{~m}$ thick layer of porous material with a flow resistivity of 1000 $\mathrm{kg} \mathrm{m}^{-3} \mathrm{~s}^{-1}$ is considered as a reference example for the proposed methods throughout this thesis (see Chap. 5). For these given considerations, the resulting normalized complex impedance in respect to the characteristic impedance $\rho_{0} c$ is represented in Fig. 2.5. As is mentioned in Sec. 4.2.1, the results presented in this thesis are presented in a normalized frequency corresponding to a sampling frequency $f_{s}=80 \mathrm{kHz}$.

In accordance with the locally reacting impedance definition, Eq. 2.70, the theoretical reflection factor can be theoretically calculated for different angles. In this case, the angles of arrival under study $\theta$ are $0,15,30,45,60$ and 75 degrees. These theoretical reflection factors are indicated in Fig. 2.6.

In this thesis, a digital filter-based representation of both reflection factor and impedance is used. From the point of view an impedance, slight vari- 


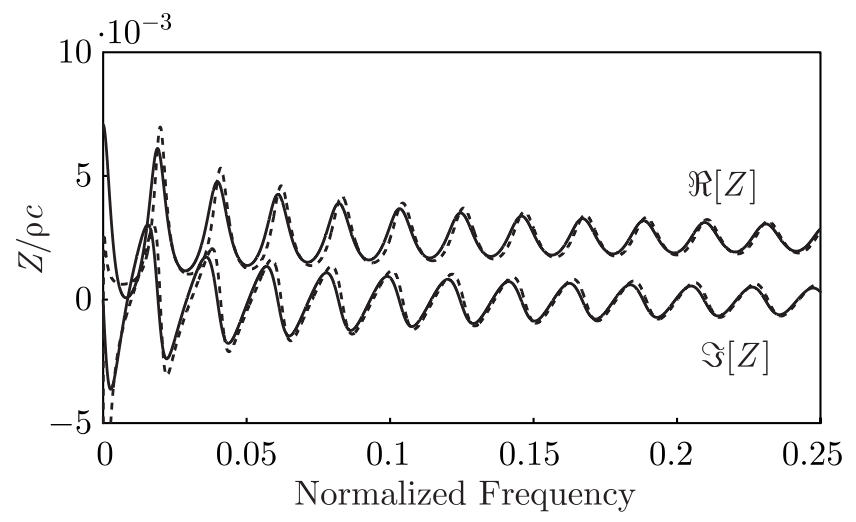

Figure 2.5. Complex impedance of a hard-backed layer of porous material, where the continuous line represents the real and imaginary part of the theoretical expression, whereas the dashed line corresponds to the approach followed during this thesis.

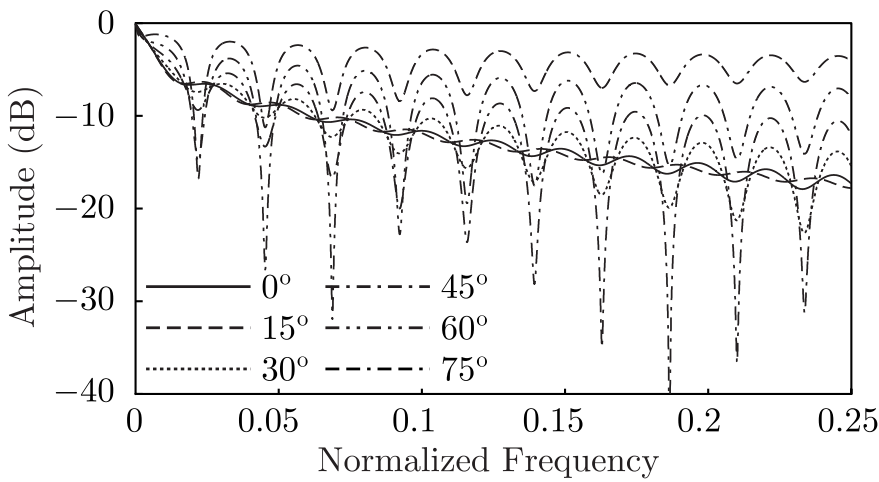

Figure 2.6. Theoretical reflection factor calculated for different angles of arrival, according to the analytical expression for a locally reacting impedance assumption.

ations in its modeling could produce evident changes on the reflection factor obtained through Eq. 2.70. For instance, during this thesis (see Chap. 5), the impedance is approached using the Yule-Walker [Friedlander and Porat, 


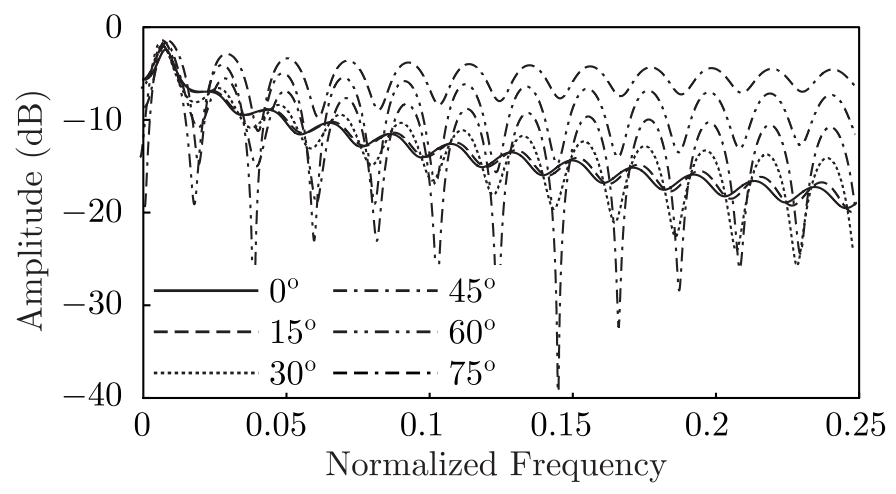

Figure 2.7. Reflection factor calculated from the spectrum response of a Yule-Walker approach of the impedance for different angles of arrival, according to the analytical expression for a locally reacting impedance assumption.

1984 ] in order to synthesize a 60 th order digital filter ${ }^{2}$. From the frequency response of that filter, it could be observed some differences in the reflection factor at different angles of arrival (see Fig. 2.7).

From this point, what should be noted is how in case to use a digital filter representing the impedance, the reflection factors used as a referenced must be the ones obtained from the approached impedance spectrum. Furthermore, one must be careful with this, since when a reflection factor is used as as boundary condition, it must be take into account the differences between the theoretical reflection factor for an analytical impedance and the one coming as a consequence of the spectrum of the digital filter approach of the impedance.

This premise would be assumed along this thesis and it should be specified which option is used at each particular example.

\footnotetext{
${ }^{2}$ This method as been selected for simplicity using a Matlab ${ }^{\circledR}$ code, although some experiments using more efficient methods such as [Ramos and López, 2006] are carrying out with successful results.
} 


\subsubsection{Sound source modeling}

In this section, some fundamental concepts of sources are reviewed, focused on the directive properties of sound power sources. Furthermore, some basic directive sources are presented, called multipoles, since they can be seen as an orthogonal basis for more complex and realistic directive sources.

\subsubsection{Directive sources}

Real-world sources are far from the basic sources introduced previously in Sec. 2.3.2. The sound power of sources is not usually spread evenly. This means, the intensity vector depends also on polar and azimuth angles.

Now, let us consider a source with a non-spherical symmetry. At a large distance from the source, the acoustic variables locally resemble a plane wave propagating with speed of sound $c$ away from the source. Then, it can be written

$$
\begin{aligned}
p(\mathbf{r}, t) & \propto g(t-\|\mathbf{r}\| / c, \theta, \phi), \\
\mathbf{u}(\mathbf{r}, t) & \propto \frac{p(\mathbf{r}, t)}{\rho_{0} c} \hat{\mathbf{n}}_{r},
\end{aligned}
$$

where $\theta$ and $\phi$ denote polar and azimuthal angles in spherical coordinates.

Considering $g$ as a sinusoidal function of time, it is reasonable to consider the next approximate simplifications [Pierce, 1994]:

$$
\begin{aligned}
p(\mathbf{r}, t) & =\frac{1}{r} g(t-\|\mathbf{r}\| / c, \theta, \phi), \\
\mathbf{u}(\mathbf{r}, t) & =\frac{p(\mathbf{r}, t)}{\rho_{0} c} \hat{\mathbf{n}}_{r}, \\
\mathbf{i}_{\mathrm{av}} & =\frac{J(\theta, \phi)}{\|\mathbf{r}\|^{2}} \hat{\mathbf{n}}_{r}, \\
J(\theta, \phi) & =\frac{1}{\rho_{0} c T_{a}} \int_{t_{0}}^{t_{0}+T_{a}} g^{2}(t, \theta, \phi) d t,
\end{aligned}
$$

with $T_{a}$ being a very long time period containing an ample number of half periods. The first two expressions are, in fact, not restricted to periodic signals. The function $J(\theta, \phi)$ describes the radiation pattern of the source, e.g. acoustic power radiated per unit solid angle. 


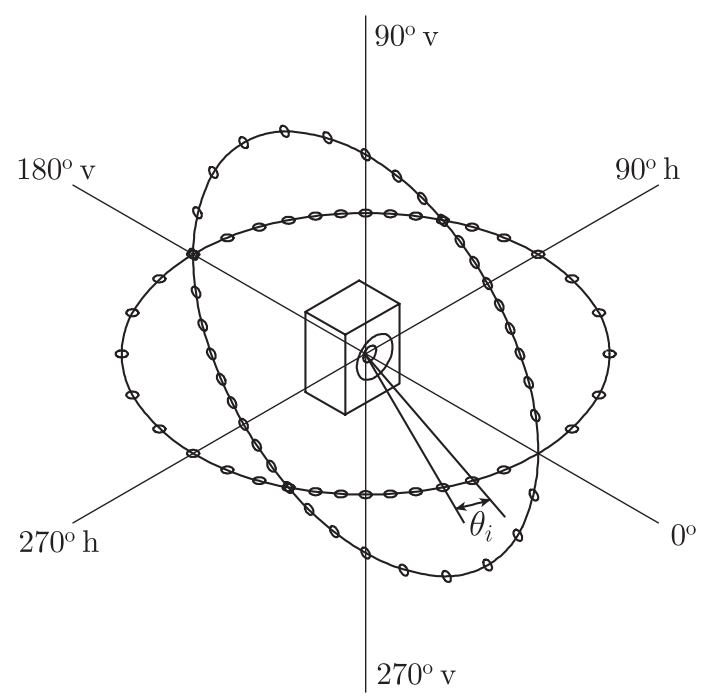

Figure 2.8. Measuring points in order to measure the directivity in $(\theta, \phi)$ points.

These equations indicate the spherical spreading law is not restricted to spherically symmetric sources, allowing the use of these concepts in real sources.

The radiation pattern has a measurable consequence, the source directivity $D_{s}(\theta, \phi)$. This function is related to the source in far-field conditions and constant vorticity assumption as

$$
\begin{aligned}
& \mathbf{u}(\mathbf{r}, t)=D_{s}(\theta, \phi) \mathbf{u}_{o}(\mathbf{r}, t), \\
& p(\mathbf{r}, t)=D_{s}(\theta, \phi) p_{o}(\mathbf{r}, t),
\end{aligned}
$$

where $\mathbf{u}_{o}(\mathbf{r}, t)$ and $p_{o}(\mathbf{r}, t)$ are the omnidirectional particle velocity and pressure of the source. Figure 2.8 shows an example of the set-up for measuring the directivity of a loudspeaker inside a box.

The directivity and the radiation pattern is related through

$$
J(\theta, \phi)=\left\|D_{s}(\theta, \phi)\right\|^{2} J_{o}
$$


where $J_{o}$ is the radiation pattern in the far-field for an omnidirectional source, defined as

$$
J_{o}=\frac{1}{\|\mathbf{r}\| \rho_{0} c T_{a}} \int_{t_{0}}^{t_{0}+T_{a}} g^{2}(t-\|\mathbf{r}\| / c) d t,
$$

where function $g$ does not depend on azimuth or elevation since $J_{o}$ is defined for an omnidirectional source.

Let us consider an sinusoidal wave, where since the omnidirectional pressure (and also the particle velocity) is constant in angle, and the pressure reference $p(\mathbf{r}, t)$ is measured in far field conditions, the directivity can also be seen as

$$
D_{s}(\theta, \phi)=\left\|\frac{P(\omega, \theta, \phi)}{P_{o}(\omega)}\right\|,
$$

giving a new definition of directivity as the module of the pressure in an angular position $(\theta, \phi)$, divided by a reference pressure. This reference used to be obtained as the maximum pressure produced by the source and corresponding to symmetry axis.

Since the directivity is now defined as the ratio of two frequencydependent functions, it is straightforward to consider the directivity as a function of the angle. This allows us to define the directivity diagram as a graphical representation of the directivity for a particular frequency as a function of each one of the angles (see an example in Fig. 2.9).

\subsubsection{Some basic directive sources: from monopoles to quadripoles}

Any spherically symmetric source of sound, and particularly a point source, with an angular frequency $\omega$ and situated in position $\mathbf{r}_{s}$ radiates an outgoing spherically symmetric wave with a complex pressure amplitude represented as

$$
P(\mathbf{r}, \omega)=S_{m}(\omega) \frac{e^{j k\left\|\mathbf{r}-\mathbf{r}_{s}\right\|}}{\left\|\mathbf{r}-\mathbf{r}_{s}\right\|} .
$$

where the function $S(\omega)_{m}$ is defined as monopole amplitude since the terms point source and acoustic monopole are synonyms. The monopole amplitude is calculated for $k\left\|\mathbf{r}-\mathbf{r}_{s}\right\| \gg 1$ as 


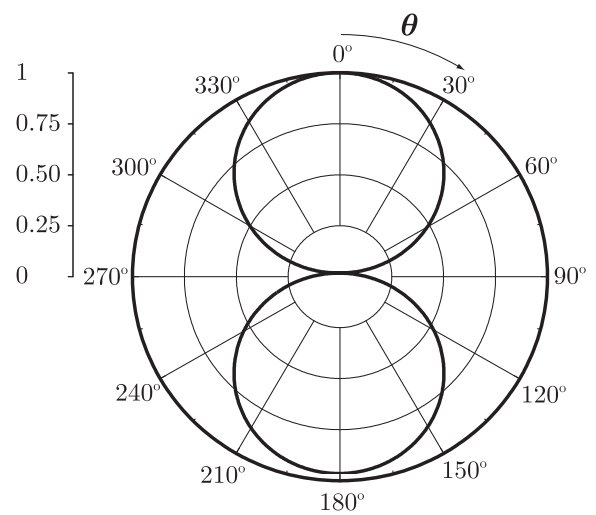

Figure 2.9. Example of a directivity diagram. The directivity has been represented as a scalar function and as a function of the azimuth angle $\theta$.

$$
S_{m}(\omega)=\frac{Q \rho_{0} c k}{4 \pi}
$$

where $\mathrm{Q}$ is a constant, termed the complex source strength and represents the volume of fluid displaced by the source.

The monopole pressure satisfies the Helmoltz equation, except in the source position, leading to a generalized function

$$
\nabla^{2} P(\mathbf{r}, \omega)+k^{2} P(\mathbf{r}, \omega)=-4 \pi S(\omega)_{m} \delta\left(\mathbf{r}-\mathbf{r}_{s}\right)
$$

To obtain the directivity of a monopole is straightforward, since it radiates spherically symmetric and then $D_{s}(\theta, \phi)=1$.

A more complex source can be obtained as a combination of those monopoles with the proper choice of the monopole weight and at a different position [Russell et al., 1999] (see Fig. 2.10). This is the case of the dipole, consisting of two monopoles of equal source strength but opposite phase, and separated by a small distance $d$ (such that $k d \gg 1$ ). The far-field expression for the amplitude of a sinusoidal wave is calculated through 


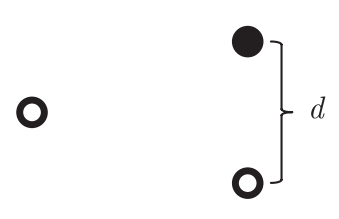

(a) (b)

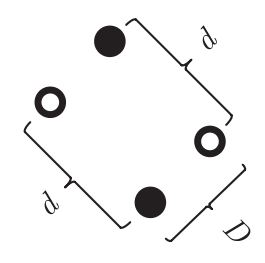

(c)

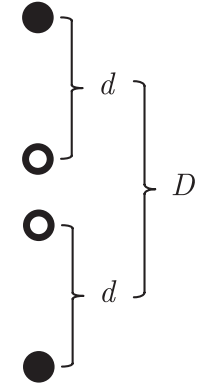

(d)

Figure 2.10. Multipole distribution to conform: (a) monopole, (b) dipole, (c) lateral quadripole and (d) longitudinal quadripole. White and black circles have an opposite phase.

$$
P(\mathbf{r}, \theta, \omega)=\frac{Q \rho_{0} c k}{4 \pi} \frac{e^{j k|| \mathbf{r}-\mathbf{r}_{s} \|}}{\left\|\mathbf{r}-\mathbf{r}_{s}\right\|} k d \cos \theta,
$$

which directivity is expressed by the term $D_{s}(\theta, \phi)=\cos \theta$ (see Fig. 2.11b))

A quadrupole consist of two identical dipoles with opposite phase and separated by a small distance $D$. For a lateral quadrupole source, the dipole axes do not lie along the same line (see Fig. 2.10c)), while for a longitudinal quadrupole source, the dipole axes do lie along the same line(see Fig. 2.10c)). For the lateral quadrupole, the complex amplitude is calculated through

$$
P(\mathbf{r}, \theta, \omega)=\frac{Q \rho_{0} c k}{4 \pi} \frac{e^{j k|| \mathbf{r}-\mathbf{r}_{s} \|}}{\left\|\mathbf{r}-\mathbf{r}_{s}\right\|} 4 k^{2} d D \cos \theta \sin \theta,
$$

which directivity is expressed by $D_{s}(\theta, \phi)=\cos \theta \sin \theta$ (see Fig. 2.11c)); whereas the longitudinal quadrupole has a complex amplitude expressed as

$$
P(\mathbf{r}, \theta, \omega)=\frac{Q \rho_{0} c k}{4 \pi} \frac{e^{j k|| \mathbf{r}-\mathbf{r}_{s} \|}}{\left\|\mathbf{r}-\mathbf{r}_{s}\right\|} 4 k^{2} d D \cos ^{2} \theta,
$$

which directivity is expressed by $D_{s}(\theta, \phi)=\cos \theta^{2}$ (see Fig. 2.11(d)). 


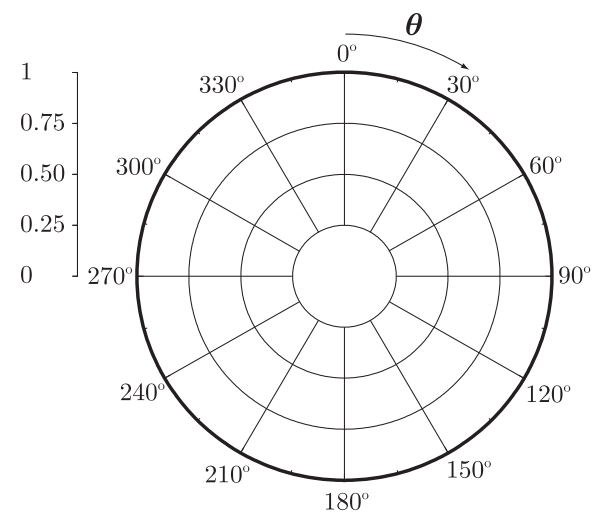

(a)

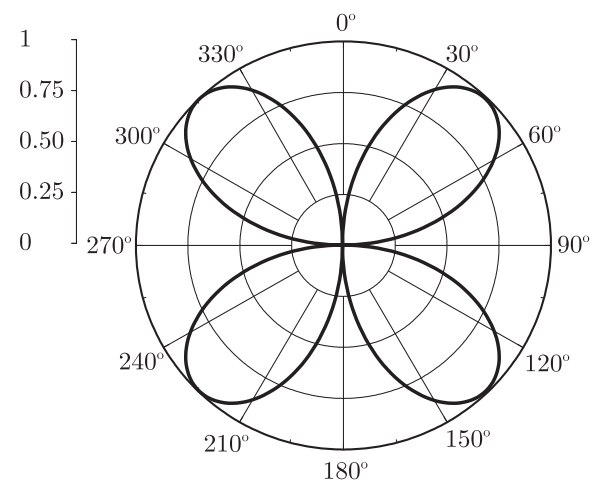

(c)

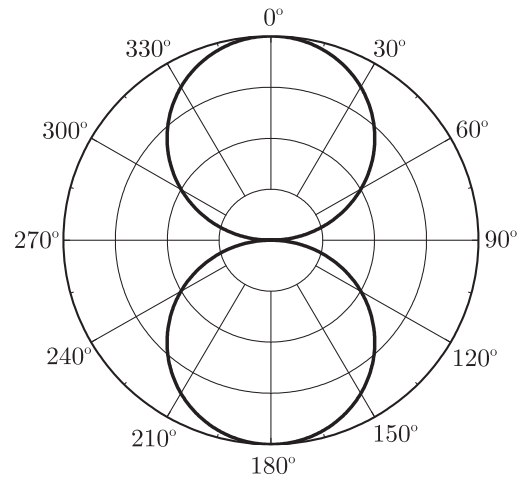

(b)

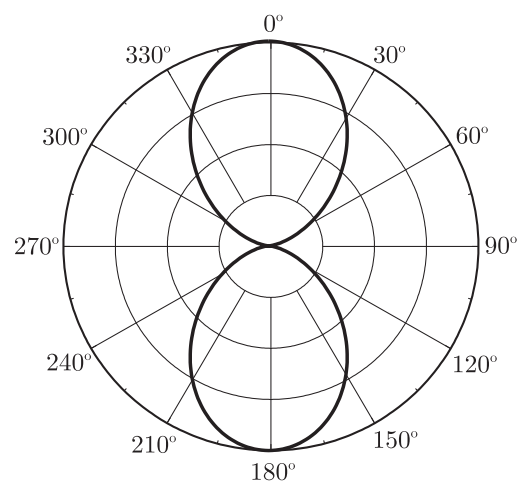

(d)

Figure 2.11. Directivity diagram of some multipole distributions: (a) monopole, (b) dipole, (c) lateral quadrupole and (d) longitudinal quadrupole. 
In fact, dipoles, quadrupoles, ..., (from now on multipoles) are also a solution to the wave equation expressed in spherical coordinates [Williams, 1999]. Indeed, radiation from bodies which are located at the origin and which are of finite extent can be characterized by a sum of multipoles:

$$
P(\mathbf{r}, \theta, \phi, \omega)=\sum_{n=0}^{\infty} \sum_{m=-n}^{n} C_{m n} h_{n}^{(1)}(k\|\mathbf{r}\|) Y_{n}^{m}(\theta, \phi),
$$

where the pressure is evaluated at a distance $\|\mathbf{r}\|$ greater than the largest radial extent of the source. The function $h_{n}^{(1)}$ is the spherical Hankel function of the first kind of order $n$ and $Y_{n}^{m}(\theta, \phi)$ is the spherical harmonic of order $n$ and degree $m$ [Williams, 1999]. From the properties of the spherical Hankel functions [Abramowitz and Stegun, 1970], it is stated that

$$
h_{n}^{(1)}(k\|\mathbf{r}\|) \propto e^{j k\|\mathbf{r}\|}
$$

representing an outgoing wave. The coefficients $C_{m n}$ represent the weighting in order to obtain a sound pressure field distribution, and they can be considered as frequency-dependent.

Let us define a spherical harmonic $Y_{n}^{m}(\theta, \phi)$ such as

$$
Y_{n}^{m}(\theta, \phi) \equiv \sqrt{\frac{(2 n+1)}{4 \pi} \frac{(n-m) !}{(n+m) !}} P_{n}^{m}(\cos \theta) e^{j m \phi}
$$

where $P_{n}^{m}$ is the associated Legendre function given by two integer $m$ (degree) and $n$ (order) [Williams, 1999].

There is not a one-to-one equivalence between spherical harmonics and multipole expansion, but also, the multipoles can be expressed for particular spherical harmonics [Williams, 1999]. Multipoles are constructed from distributions of points sources, infinitesimally close to the origin, of equal amplitudes but opposite phase. Whereas spherical harmonics form an orthogonal basis, multipoles do not. In example, $Y_{0}^{0}(\theta, \phi)$ corresponds with monopole (see Fig. 2.12); different dipole orientations are achieved through $\Re\left[Y_{1}^{1}(\theta, \phi)\right], \Im\left[Y_{1}^{1}(\theta, \phi)\right]$ and $Y_{1}^{0}(\theta, \phi)$ (see Fig. 2.13); on the other hand, some quadripoles are obtained with multipoles $\Im\left[Y_{2}^{2}(\theta, \phi)\right], Y_{2}^{0}(\theta, \phi)$ and $\Re\left[Y_{2}^{1}(\theta, \phi)\right]$ (see Fig. 2.14). 


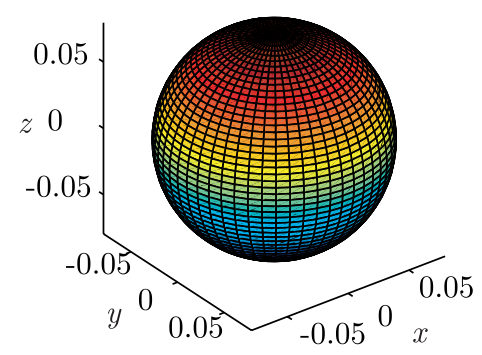

Figure 2.12. Monopole directivity obtained through spherical harmonic $Y_{0}^{0}(\theta, \phi)$.

\section{$\Re\left[Y_{1}^{1}\right]$}

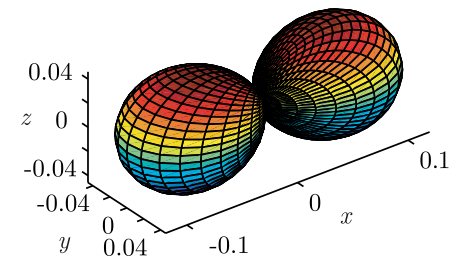

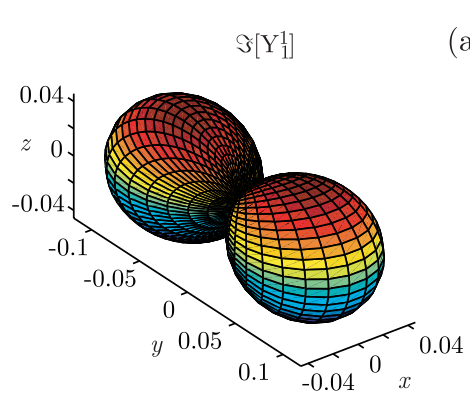

(b) (a)

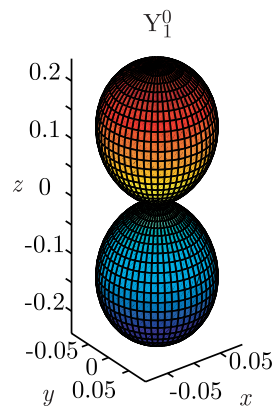

(c)

Figure 2.13. Dipole directivities obtained through spherical harmonic a) $\Re\left[Y_{1}^{1}(\theta, \phi)\right]$, b) $\Im\left[Y_{1}^{1}(\theta, \phi)\right]$ and c) $Y_{1}^{0}(\theta, \phi)$. 


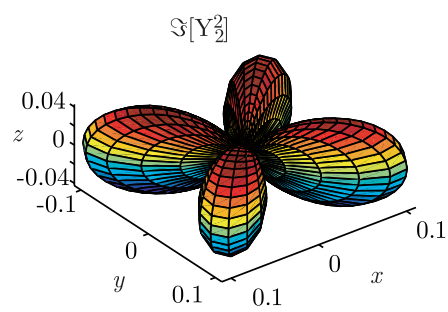

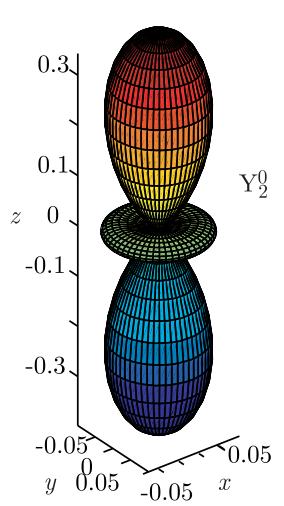

(b)

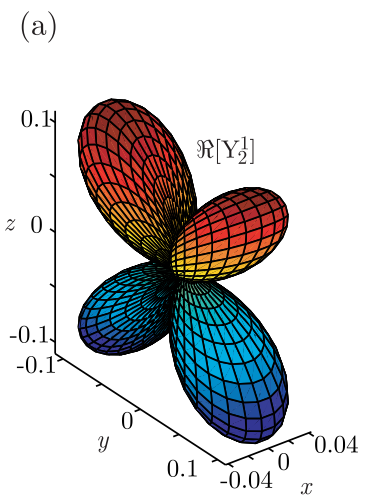

(c)

Figure 2.14. Quadrupole directivities obtained through spherical harmonic a) $\Im\left[Y_{2}^{2}(\theta, \phi)\right]$, b) $Y_{2}^{0}(\theta, \phi)$ and c) $\Re\left[Y_{2}^{1}(\theta, \phi)\right]$.

\subsection{Room acoustics as an inhomogeneous bound- ary value problem}

After presenting the most relevant acoustic foundations for the present thesis along this chapter, they can be summarized just as an inhomogeneous boundary value problem [Polyanin and Zaitsev, 2003], as follows:

$$
\begin{aligned}
& \nabla^{2} p(\mathbf{r}, t)-\frac{1}{c^{2}} \frac{\partial^{2} p(\mathbf{r}, t)}{\partial t^{2}}=s\left(\mathbf{r}_{s}, t, \theta, \phi\right) \text { in } D \subset \mathbb{R}^{3}, \\
& Z(\omega) \frac{\partial P(\mathbf{r}, \omega)}{\partial \hat{\mathbf{n}}}+j \omega \rho_{0} P(\mathbf{r}, \omega)=0 \text { on } \partial D,
\end{aligned}
$$


where $D$ denotes the domain of propagation, the region in which the wave propagates and $\partial D$ the boundary region.

Equation 2.94 corresponds to the inhomogeneous wave equation, since it includes the source term $s$ which is different to 0 at position $\mathbf{r}_{s}$. The source term also has a dependence on the azimuth and elevation angle, denoting a certain directivity.

Regarding the boundary condition (Eq. 2.95), as said during Sec. 2.4.1, this thesis is only focused on the most commonly relevant boundary condition, namely the impedance boundary condition. In this equation [Kuttruff, 4th edition, 2000], and throughout, $\partial / \partial \hat{\mathbf{n}}$ denotes the normal derivative on the boundary, i.e. the rate of increase in the direction $\hat{\mathbf{n}}$, where $\hat{\mathbf{n}}(\mathbf{r})$ denotes the unit normal at $\mathbf{r} \in \partial D$, directed into $D^{3}$.

\subsection{Discussion}

In this chapter, some of the fundamentals of the mathematics and physics involving the sound wave propagation are introduced. The particular distribution of the sound quantities such as the pressure and particle velocity components, determines the perception of the acoustical properties. It could be possible to mathematically determine those sound field properties through a sound wave equation.

A first step before entering into the acoustic of a room is to model the sound propagation in an unbounded medium. Once the equations involved in the sound propagation are determined, the room acoustic is defined as the consequence of a sound field in a bounded area. The boundary conditions in room acoustic are determined by the absorbing and reflecting properties of the walls. Along this chapter only smooth walls are considered, and without loss of generality, a locally reacting impedance is the followed model for specular reflection. It allows the definition of a wall property through a unique impedance, independent of the amplitude and phase distribution of

\footnotetext{
${ }^{3}$ It should be noted how Eq. 2.94 is expressed in the time domain, whereas Eq. 2.95 is in the frequency domain. In most scientific texts both equations are expressed in the frequency domain in order to keep a coherent formulation, since Eq. 2.95 must be expressed in this domain because impedance is a frequency-based concept [Kuttruff, 4th edition, 2000]. However, the core of this thesis is based on time domain methods for solving wave equation. Then, the author of this thesis suggests that these expressions are consequent with the rest of the text exposed on this thesis.
} 
the incident wave over the surface.

Regarding the sound source modeling, apart from their position and energy, spatial source properties are far from idealized radiation patterns as plane and spherical (point) sources. It is possible to characterize real sources through their directivity properties, which are angle dependent, having information how the energy is spread from the source and which dependence can be considered, with no loss of generality, as frequencydependent.

Thus, any particular sound field can be represented as an inhomogeneous boundary value problem, where a partial differential equation (wave equation) could be solved for some particular boundary conditions and a determined source term, that being what differentiates one room from another. 


\title{
Room Acoustics Simulation Techniques
}

\begin{abstract}
DURING THE PREVIOUS CHAPTER, some of the mathematical and physical concepts regarding the room acoustic have been presented. As has been previously mentioned, an inhomogeneous boundary value problem is the starting point to predict and analyze a particular sound field in an enclosure (see Sec. 2.5). However, with the exception of some particular (and very simple) cases, these inhomogeneous boundary value problems are unsolvable analytically. For that reason, the room acoustic prediction requires obtaining an approximation of the sound field through several physical simplifications and to define computer algorithms for solving the problem in a reasonable computing time [Schroeder, 1973]. Depending on what possible simplifications are carried out, some of the different room acoustic simulation techniques arise.
\end{abstract}

\subsection{Introduction}

In this chapter, some of the main computer simulation techniques for room acoustic simulation are revisited, with particular emphasis on the ability to incorporate source modeling and boundary conditions. In this thesis, the classification followed is based on geometrical and wave methods. Due to the 
nature and scope of this thesis (see Chap. 1), the wave-based methods are also subdivided in two large groups: in the frequency and time domain (the latter, also known as discrete-time modeling or methods). Savioja [Savioja, 1999] also included statistical methods, but since they do not model the temporal behavior of the sound field, they are far from the aim of this thesis.

It is the purpose of this chapter not to deal with a deep overview of the current state-of-the-art in room acoustic modeling, but also to give some of the advantages and disadvantages of some of the most representative techniques, with the aim of justifying the use of the discrete-time methods.

The current chapter is organized as follows: Sec. 3.2 presents some of the most important geometrical methods, focused on a brief overview of the ray-tracing and image-source method. The next two sections deal with wave-based methods. The first one concerns frequency-based wave methods with a generic sight over the two most used methods for room acoustic simulation: finite and boundary element methods. In Sec. 3.4, as this thesis is mainly focused on time-based wave methods (or discrete-time methods), a deeper overview of the state-of-the-art is introduced, justifying reasons to contribute to these methods. Finally, the chapter is summarized.

\subsection{Geometrical methods}

The key point in these methods is to assume the sound propagation as a ray. This simplification can considerably decrease the computational cost since a PDE problem is directly transformed into an algebraic and/or geometric problem. Geometrical methods make the assumption that sound wavelengths are significantly smaller than the size of obstacles found in their path. Thus, they are valid only for high-frequency sounds. This means some sound propagation phenomena in low frequencies, such as diffraction, are not considered under these approximations.

Regarding the boundary conditions, at first sight, these methods assume specular reflections, since the angle of reflection is the same as the angle of arrival. It could also include the absorption issue in a frequencydependent manner according to the impedance/absorbing factor of the wall (see Sec. 2.4.1.1) and also including sound propagation absorption. 
A considerable disadvantage of these methods lies in the inability to include phenomena such as interference, diffusion and diffraction, since they are phenomena which appear when wavelengths are similar to the size of obstacles. However, some advances have appeared to include these effects and produce highly realistic results.

Two main algorithms have emerged from the geometrical method's assumptions: the ray-tracing and the image-source method. From this moment on, many variations of these methods have emerged in order to improve their computational cost, flexibility or to include some of the sound propagation characteristic properties, such as diffraction and diffusion [Cox et al., 2006]. Although being far from the scope of this thesis, it is worth mention that recently a unified integral equation generalizing those methods in an unique formulation has been proposed [Siltanen et al., 2007]. In the next subsections, these algorithms are overviewed.

\subsubsection{Ray-tracing method}

The ray-tracing method is a general technique from geometrical optics of modeling the path taken by a wave by following rays as they interact with surfaces. This method arises from optical physics [Lipson et al., 1995], with important applications in the computer graphics field [Whitted, 1980, Glassner, 1989] and communications [McJown and Hamilton, 1991, Cátedra et al., 1998, Coleman, 1998, Ji et al., 2001].

The first attempt to use the ray-tracing technique for room acoustic simulation was applied by Krokstad et al [Krokstad et al., 1968]. They propose finding propagation paths between a source and a receiver by generating rays emanating from the source position and following them through the environment until a set of rays has been found that reach the receiver. As mentioned above, the initial tendency of this algorithm is to consider only specular reflections, where in each incidence, the incident ray is filtered with its absorbing coefficient (non-frequency or frequency dependent), and reflected with the same angle of incidence [Kulowski, 1985]. Thus, it could be considered that ray-tracing allows simulation of locally reacting impedances (see Sec. 2.4.1.3). However, several improvements of the algorithm have included diffusive surfaces [Lam, 1996, Embrechts, 2000] and edge diffraction [Svensson et al., 1999]. Ray-tracing applies the Monte Carlo simulation technique to sample these reflection paths and thus it gives a statistical result [Halton, 1970]. By this technique higher order reflections can 
be searched for, though there are no guarantees that all the paths will be found.

Regarding the sources, in principle, the way sound rays are emitted can be either randomized or predefined as a function of the directional characteristics of the sound source [Savioja, 1999]. Therefore, it must be ensured that the generation is almost uniform on the surface of a spherical source [Farina, 1995]. The simple assumption of three random generators for the three components of the vector direction of the ray is not completely correct, as that produces a "cube of rays" instead of a sphere. It is possible to cut away the corners of the cube, discarding each vector with a modulus greater than one [Farina, 1995]. If each ray is weighted by an angle dependent function, it is possible to include directivity properties to the source with no increase of computational cost.

The listener positions cannot be considered as a point, since an infinitely small point cannot detect an infinite small ray. For this reason, the receiver should have a finite size. The listeners are typically modelled as spheres or cubes, but the listeners may also be planar. In theory, a listener can be of any shape as long as there are enough rays to penetrate the listener to achieve statistically valid results. In practice, a sphere is in most cases the best choice, as it provides an omnidirectional sensitivity pattern and it is easy to implement.

Some examples of its application and comparison with measurements can be found in [Hodgson, 1989, Tsingos et al., 2002]. A software example of the use of the ray-tracing method is CATT-Acoustic ${ }^{1}$.

\subsubsection{Image-source method}

From a computational point of view the image source method is also a raybased method. The concept of image sources has been applied to various field problems such as electromagnetic wave propagation [Balanis, 1997]. In acoustics, the first contribution on the room acoustic field was presented by Allen and Berkley [Allen and Berkley, 1979] and extended to arbitrary polyhedra by Borish [Borish, 1984].

The concept is based on the principle that a specular reflection can be represented as a sound source located outside the physical boundary, radiating in free space. It computes specular reflections paths by considering

\footnotetext{
${ }^{1}$ URL: http://www.catt.se.
} 
virtual sources generated by mirroring the location of the audio source, over each boundary surface of the environment.

The primary advantage of image source method is their robustness. They guarantee that all specular paths up to a given order of reverberation time are found. Also the listener can be modeled as a point instead of a volumetric object. Thus, the results are more accurate if the order of the reflections is high enough.

However, image source methods model only specular reflection, and their expected computational complexity grows exponentially. Thus, it is an important matter to try to reduce this complexity i.e., checking visibility. The visible surfaces to each image source can be calculated by beam tracing [Funkhouser et al., 1998]. Each beam is a pyramidal frustum, whose tip is the image source and whose top is the reflecting surface. Only the surfaces inside these volumes, which are faced toward the image source, must be taken account. For this reason image source models are usually only used for simple rectangular rooms or in such cases where low order reflections are sufficient [Rindel, 1995].

This method has been implemented in commercial softwares such as CARA (Computer Aided Room Acoustics) ${ }^{2}$.

\subsubsection{Hybrid and alternative geometrical methods}

A possible improvement of those models is to create hybrid models, in which ray tracing and the image source method are applied together. Typically, early reflections are calculated with image sources due to their accuracy in finding reflections paths, and later reflections are handled with ray tracing. An example of this method is the ODEON software [Naylor, 1993] ${ }^{3}$, initially developed in the Technical University of Denmark.

Due to the highly elevated computational cost and other implementation problems of these geometrical methods, an increasing number of alternative methods has appeared during last few years, being more or less inspired by the previous ones. However, it should be mentioned all of them share the same problematic regarding to the low frequencies approaches as in ray tracing and image-source methods. Some of the most important methods are commented in this section.

\footnotetext{
${ }^{2}$ URL: http://www.cara.de.

${ }^{3}$ URL: http://www.odeon.dk
} 
An alternative method is the beam tracing method [Funkhouser et al., 2004]. It arises as an improvement to the ray tracing, since rays have no thickness, and it uses beams. Beams are shaped like unbounded pyramids and each beam represents an infinite number of potential ray paths emanating from the source location. It does not suffer from the sampling artifacts of ray tracing [Lehnert, 1993], nor the overlap problems of cone tracing [Vian and van Maercke, 1986]. Another alternative is the pyramid tracing, suited to room acoustics and outdoor calculations [Farina, 1995]. The main advantage of Pyramid Tracing over other diverging beam tracers is the fact that pyramids perfectly cover the surface of a spherical source, while cones cause overlapping or uncovered zones. This method has been produced the first publicly available software for acoustical simulations based on this method, RAMSETE ${ }^{4}$

Another method is the acoustic radiosity [Dalenbäck, 1996, Hodgson and Nosal, 1996, Nosal et al., 2004], that is able to handle surfaces with mixed specular and diffuse reflection properties in a very general way, based on approximate cone tracing. The specular reflections are implemented in the ray tracing manner, and each ray striking a diffusing surface should ideally create a new source that spawns a set of secondary rays, each of which continues as any other ray but from a new origin. The process should then be continued until the echogram is saturated.

Finally, acoustic sonel mapping [Kapralos, 2006] is an application of the photon mapping algorithm used in computer graphics for room acoustic simulation. Acoustic sonel mapping is a two-pass particle-based, probabilistic global method developed in order to determine the sound field at any point in a scenario. In the first pass, "sonel" (the analogue to photons, the basic quantity of light, when considering the visual photon mapping method) are emitted from each sound source and traced through the scene until they interact with a surface. When sonel encounter a diffuse surface, they are stored in a structure called a sonel map. In the second stage, the scene is rendered using the information provided by the previously collected sonel map to provide a quick estimate of the diffuse reflected sound field. A distribution of ray tracing is employed to model specular effects. Sonel mapping is independent of the scene geometry, thereby allowing for the sound field simulation of arbitrary complex scenes to be computed. In addition, it can handle complex interactions between sound and a surface, including pure

\footnotetext{
${ }^{4}$ URL: http://www.ramsete.com.
} 
specular, pure diffuse and glossy reflections and any combination of them.

\subsection{Wave methods in the frequency domain}

In this section two of the most important wave-based methods in the frequency domain are presented: Finite Element Method (FEM) and Boundary Element Method (BEM). These methods have been widely used in disciplines such as structural mechanics [Courant, 1943, Dominguez, 1993] and electromagnetism [Jin, 2002, Chen and Zhou, 1992]. In the acoustic literature, their application has an innumerate number of applications whose main scope is the analysis of the resonance of acoustic systems [Ihlenburg, 1998, Wrobel and Aliabadi, 2002]. This discretization is defined for discrete frequencies, giving as a result a steady-state sound pressure distribution.

Due to the discrete frequency-based nature of these algorithm, they are not usually used for room acoustic simulation, but they are very useful and accurate for eigenvalue analysis of the enclosures. Moreover, the use of FEM or BEM in room acoustical problems is (at least for the majority of rooms larger than e.g. vehicle enclosures) up until now limited to a frequency range that is considerably smaller than the range of commonly used room acoustical analyzes up to high frequencies. The limiting factor for these methods is the required discretization of the volume or surface in elements of a size small enough to reconstruct the wave in magnitude and phase at every point of the discretization domain.

Despite of these limitations, there exist a few applications of these methods in room acoustics which makes it worthy to review them in most parts of the literature related. From a general point of view, regarding the boundary conditions and source modeling, wall absorption is not a simple task [Wright, 1995], but an unsolved problem is the use of directive sources [Svensson, 2004]. In the next sections, both methods are presented in some detail.

\subsubsection{Finite Element Method}

FEM is primarily used when only the interior acoustic field of an enclosure is to be computed. The main advantage of this method lies in the ease of handling problems involving complex geometries and inhomogeneous media. The systematic generality of the method makes it possible to build general 
purpose computer programs. The method consists of discretizing the space under analysis by using a set of basis functions. These basis usually conform a tessellation of the whole space. This representation by elements creates matrices that are large but sparsely filled. Each of these elements only interacts with the elements directly adjacent to it.

The finite element analysis of any problem involves four basic steps:

- discretizing the solution region into a finite number of subregions or elements.

- deriving governing equations for a typical element.

- assembling of all elements in the solution region, and

- solving the system of equations obtained.

Although there exists a very high number of FEM applications in acoustics, the choice of this method for room acoustic simulation could give a set of important handicaps. The main handicap lies on the frequency domain: it requires a simulation for each frequency and since the room acoustic properties are usually not constant with the frequency, it will considerably increase the computational effort. But this is not only limitation of this method: it requires a very small element size for accurately model in the entire frequency bandwidth. Six or seven elements per wavelenght are required as sufficient sampling rate [van der Geest and McChulloch, 1998, Murphy, 2000]. In an example reported by Murphy [Murphy, 2000], if an element is $0.5 \mathrm{~m}$ long, with $c=343 \mathrm{~ms}^{-1}$, the maximum frequency that can be calculated with any reasonable accuracy is approximately $114 \mathrm{~Hz}$. In a 3$\mathrm{D}$ simulation, doubling the frequency the number of elements are multiplied by eight (more details will be given at Sec. 4.6).

Despite of these limitations, in the room acoustics field, some analysis of rectangular rooms through FEM have been provided by Wright [Wright, 1995] and Savioja et al. [Savioja et al., 1996a]. On the other hand, more realistic rooms have recently been analyzed giving a fair agreement among simulation and measurements in small [Pietrzyk and Kleiner, 1997] and large [Ahnert et al., 2006] rooms. 


\subsubsection{Boundary Element Method}

Since FEM is fairly a very computational expensive method, BEM arises as an alternative method which considerably reduces the computational cost, since only boundaries are discretized. BEM attempts to use the given boundary conditions to fit boundary values into the integral equation, rather than values throughout the space defined by a partial differential equation. Once this is done, in the post-processing stage, the integral equation can then be used again to calculate numerically the solution directly at any desired point in the interior of the solution domain. It allows a simple and accurate modeling of problems involving infinite and semi-infinite domains. The matrix implementation of the method gives a full matrix, i.e. every element of the matrix is non-zero [Kirkup, 1998].

As well as FEM, a few examples of its applications to room acoustic simulations can be found. Those examples are found in [Bai, 1992] and [Osa et al., 2006], where the latter analyzes large hall effects through this method.

\subsection{Wave methods in the time domain}

So far, several methods have been reviewed in this chapter: geometrical methods provide simple algorithms but their assumptions are valid only for high frequencies, whereas frequency-domain wave-based methods analyze the steady-state of a room and since they are using the wave equations as a starting point, they provide a high accuracy in their results. Then, it should be desirable for room acoustics simulation to use methods as accurate as frequency-domain wave-based methods but allowing transitory analysis; on the other hand, flexibility and more simple conceptually such as geometrical methods, but with a higher accuracy at low frequencies, is a main aim for room acoustic simulations.

The wave-based in the time domain, or discrete-time methods arise as suitable alternative methods, since they are based into discretize the wave equation providing a considerable accuracy, especially at low frequencies; furthermore, geometries are more simple to define than FEM or BEM, since a constantly regular polyhedron (in a $3 \mathrm{D}$ simulation) can be used during grid definition (see Appendix B). 
These methods share a common definition: they discretize the sound propagation mathematical expressions not only in space, but also in time ${ }^{5}$, giving a recursive algebraic expression which is updated in each time step for every discretized point. Since the first works in the room acoustic field of Botteldooren [Botteldooren, 1995] and Savioja [Savioja et al., 1994] in the middle of the nineties, an increasing number of publications and works related in this topic have arisen, where the finite-difference time-domain method (FDTD) and Digital Waveguide Mesh (DWM) are the most representative ones.

As mentioned in Chap. 1, this thesis is focused on analyzing and contributing to these methods, and is particularly centered on the FDTD and DWM methods. In this section, neither technical nor mathematical foundations are presented (they will be introduced along next chapters), but a summarized state of the art about the use of the discrete-time methods for room acoustic simulation is introduced. Furthermore, some alternative methods are briefly introduced in Sec. 3.4.2.

\subsubsection{Suitability of the discrete-time methods for room acoustic sim- ulation: a survey}

The Finite-Difference Time-Domain (FDTD) is one of the most popular and used methods to solve Maxwell's electromagnetic equation. It was proposed by Yee in 1966 [Yee, 1966] and the method consists of a staggered distribution of the discretized electric and magnetic variables. After that, a very prolific bibliography has appeared on electromagnetism [Taflove, 1988, Kunz and Luebbers, 1993, Taflove, 1995, 1998]. Since the FDTD method can be classed as a special case of the finite difference method, a standard method for solving PDEs [Strikwerda, 1989], the method has been adapted to solve other wave propagation problems, such as acoustic/aeroacoustic [Wang, 1996, Nguyen, 1996, Botteldooren, 1997, Ostashev et al., 2005, Liu and Albert, 2006] and seismology [Graves, 1996, Kristek and Moczo, 2006, Gandomi and Takenaka, 2007].

The first attempt to use the FDTD method on room acoustics was done by Botteldooren [Botteldooren, 1995]. He proposed an approach of the FDTD over the Euler and conservation of mass equation (Eq. 2.302.31 ), where the pressure and the particle velocity components are updated

\footnotetext{
${ }^{5}$ This is the reason for calling these algorithms discrete-time methods.
} 
in a similar fashion to the electromagnetic approach (more details will be seen in Sec. 4.2). At this time, Maloney and Cummings [Maloney and Cummings, 1995] demonstrated how the properties of the acoustic field simulated through the FDTD method in a cartesian grid are very similar to the electromagnetic field approach, sharing the same stability and dispersion characteristics. These approaches have considered a cartesian discretization. During following years, a few attempts of using Botteldooren's approach in the room acoustic simulation field, where the use of a quasi-cartesian grid, based on Voronoi cells [Botteldooren, 1994] and an analysis of the influence of the seat dips in the total sound fields [Lovetri et al., 1996] stand out.

In a deep review of the room acoustic simulation topic, it is easy to note how this approach is not very popular, although some examples of room sound field simulation are found in [Yokota et al., 2002, Sakamoto et al., 2002, 2005]. The main handicap of this approach lies in the computational cost, since the particle velocity is not usually computed as a result, but is also used as intermediate step in the final calculation of the pressure, which is the common variable determined as an output of the simulations. For that reason, it should be desirable to calculate directly the pressure distribution without store the particle velocity components, saving a considerable amount of computer memory.

At this point, a set of papers appeared, lead by Savioja, improving and adapting the suitability of the method to the room acoustic simulation, where the wave equation (Eq. 2.32) is directly approached through the FDTD instead of the Euler and conservation of mass equation [Savioja et al., 1994, 1996b]. With this approach, the pressure at each node is updated through the surrounding and previous nodes, and the massive storage of the particle velocities is avoided. In those works, the suitability of this method for room acoustics is demonstrated; however, the computational cost is still high to compute the total sound field of a moderate size room with the total bandwidth of the human hearing. For that reason, the limitation of this method is assumed for low frequencies [Savioja et al., 1994]. However, the constant growth of the computer power will allow us to apply these methods for room acoustic simulation in a near future.

A constant aspect in the room acoustic simulation through discrete-time modeling is the parallel growth with the digital sound synthesis through physical modeling topic [Välimäki et al., 2006] since van Duyne and Smith proposed the Digital Waveguide Mesh (DWM) [Duyne and Smith, 1993, 
1995]. Many of the advances on this topic have been inherited as an extension of the membranes and plate, being a 2D problem, to enclosures as a 3D problem [Savioja et al., 1995]. The DWM could also be seen as a particular class of finite difference method [Savioja et al., 1999], with the particularity that it uses a decomposition of the sound pressure waves based on incoming and outgoing components [Duyne and Smith, 1993] (mathematical details are shown in Sec. 4.3 ). This method has a similar formulation to the Transmission Line Matrix (TLM) method [Christopoulos, 1995, Elmasri et al., 1998], although finally it was demonstrated the equivalence between both methods [Johns, 1987, Krumpholz et al., 1995, Bilbao, 2001] ${ }^{6}$.

In room acoustic, the DWM method has taken an important advantage over the FDTD method, not only over the method itself, but also as the method denomination. Since Karjalainen and Erkut demonstrated their equivalence [Erkut and Karjalainen, 2002a, Karjalainen and Erkut, 2004], both methods have been denominated as Digital Waveguide Mesh, although their origins are different [de Poli and Rocchesso, 1998]. However, they are differentiate by the term Wave-DWM (W-DWM in the sequel) for the method proposed by van Duyne and Smith [Duyne and Smith, 1993] and Kirchhoff-DWM (K-DWM in the sequel) for Savioja's approach [Savioja et al., 1994]. During the last few years, these denominations could be very confusing since the terms FDTD (Savioja's approach) and K-DWM have been considered as equivalent, and also DWM and W-DWM; however these terms are alternated according to each one of the authors. In Chap. 4, all these methods are developed and explained with more detail, justifying when each terminology is used. In the following, and unless the contrary would be indicated, it will be only FDTD will be used for Botteldooren's approach; and the DWM acronym will be specified if it corresponds with the $\mathrm{K}$ - or W-approach.

There have been two main research lines in this topic: to correct the inherent dispersion of the mesh and the boundary conditions implementation. In the following, both research line advances so far, are summarized, giving more mathematical details in Chap. 4.

- Mesh dispersion correction: As will be shown in Sec. 4.2.1, all the

\footnotetext{
${ }^{6}$ Despite this equivalence, some authors such as Miklavcic and Ericsson have published a paper on this topic using this acronym Miklavcic and Ericsson [2004]. However, this work should be emphasized since they show some first examples of 3-D room acoustic simulation.
} 
frequencies do not travel with the same speed of sound, having a directional dependence as well. Although the FDTD and DWM were initially developed in a cartesian grid, it has been demonstrated how, due to their dispersion characteristics (both share same dispersion due their equivalence [Erkut and Karjalainen, 2002a]), they are not the most efficient [Campos and Howard, 2005] nor most accurate implementation of those methods [Murphy, 2000]; although it should be noted how the cartesian implementation is more conceptually simple and the geometries are easier to implement.

One of the most important contributions to this topic has been to propose alternative topologies to the cartesian mesh in discrete-time methods, where the triangular one for 2D problems [Fontana and Rocchesso, 1998] and the tethraedrical mesh for 3D problems [Duyne and Smith, 1995] are the most used. They are more efficient since they reduce the oversampling factor used for a giving an accuracy level [Fontana and Rocchesso, 2001].

Another option is to use a pre/post processing in the algorithm, which is able to compensate for the modifications in the speed of sound due to the grid. This process could be on-line [Fontana and Rocchesso, 2000] (being highly computationally expensive) or off-line. Since the use of the discrete-time methods are far from being implemented in real-time, the off-line processing is not a serious handicap for real implementations. The most important contributions to the off-line methods were done by Savioja and Välimäki when they proposed the use of warping techniques in interpolated meshes [Savioja and Välimäki, 1997, 1999, 2000, 2001, 2003]. These methods consist of the use of an interpolated mesh removing (mostly) the angular dependence of the dispersion. This interpolation is done by taking into account not only pressure values on the cartesian directions, but also weighted values of the ones situated in diagonal directions. After that, a pre-processing based on the use of a warping filter modifies the frequency axis in a non-uniform scaling factor, which is used in this application in order to match the different resulting frequencies with the expected ones. A similar method applied to the Botteldooren's FDTD can be found in [Wagner and Schneider, 2005].

- Boundary conditions: As has been mentioned in Sec. 2.4.1, boundary conditions are the mathematical representation of the walls and 
objects which interfere with the sound propagation and they are a deciding factor in the total pressure distribution in the enclosure. In this topic, two have been the research directions about boundary conditions:

1. Absorbing boundary conditions: once a FDTD grid is specified, the untreated boundaries act as hard walls (total reflection). In many acoustic simulations this is a serious handicap, since an infinite space is desired, e.g. scattered sound field analysis. Then, it is absolutely necessary to avoid this truncation in the analysis space through the so-called absorbing boundary conditions (ABC). The scope of these boundary conditions is to absorb all the waves arriving at the mesh boundaries and it is also desirable that they absorb waves completely independently of the arrival direction and frequency content.

To find a suitable ABC is not direct or simple. Many approaches have been proposed but a definitive solution has not been achieve yet. Most of these solutions have been developed to the Botteldooren's FDTD approach and are mostly an adaptation of the electromagnetic FDTD approach [Mur, 1981, Higdon, 1986, 1987, Bayliss and Turkel, 1980, Bayliss et al., 1982], but the perfect matched layer (PML) [Berenger, 1994] has been the most popular and effective method for this purpose. In acoustics, this method has also been adapted through an extensive number of publications [Yuan et al., 1997, Liu and Tao, 1997, Katsibas and Antonopoulos, 2004, 2007]. Since the PML method uses pressure and particle velocity variables and requires large boundary layers around the simulation area, it has not been used in the DWM method. For the DWM case, few attempts have been made [Murphy and Mullen, 2002, Kelloniemi et al., 2005, Kelloniemi, 2005b], but the results are quite far from the PML method results in the FDTD method. However, it should be noted how, in room acoustic simulation, it is not common practice to include these absorbing boundary conditions, except for accurate simulations where open windows or doors could exist.

2. Frequency dependent and non-dependent boundary conditions: In the particular case of the room acoustic simulation, the enclosure is (mostly) surrounded by walls and objects with a partic- 
ular impedance, which after each reflection on them, the total sound field is modified. According to Sec. 2.4.1, specular and diffuse reflections with different proportions appear each time a wave strikes over a surface. To simulate both kind of reflections through the FDTD or DWM method is not directly solved either. Regarding the diffuse reflections, several approaches have appear for the FDTD method [Lee and Smith, 2005, Redondo et al., 2007] and for the DWM method [Shelley and Murphy, 2005a,b, 2008]. This last contribution, made by Shelley and Murphy, gives accurate results for a given statistical distribution of the diffuse wall properties [Murphy et al., 2008b].

Unfortunately, the number of contributions for simulating specular reflections with some level of accuracy are lower. Under a LRI assumption, it is an unsolved problem for the FDTD and DWM method. Since this problem is one of the key points of this thesis, a deeper review of this topic will be carried out in Sec. 5.2.1.

Of course, these are not the only research lines on room acoustic simulation through discrete-time models, since many unsolved problems still exists: conformal methods for adapting geometries [Schneider et al., 1998b], impulse response recording for multichannel audio reproduction [Escolano et al., 2004, Southern and Murphy, 2007, 2008] .... Another important unsolved problem is to simulate directive sources, but the appropriate review is made in Chap. 6, since this is another key point of this thesis.

\subsubsection{Alternative methods in discrete-time modeling}

After carefully reading Sec. 3.4.1, one might think FDTD and DWM are the only discrete-time methods. But this is not true, since there exist some interesting and suitable methods for room acoustic simulation with very promising results. Some of them are detailed in the following.

- The Functional Transformation Method (FTM) [Trautmann and Rabenstein, 2003] has been developed for performing real-time sound synthesis of string instruments. An extension to solve room acoustic simulation problems has been proposed too [Petrausch et al., 2005b, Petrausch and Rabenstein, 2006]. Based on multidimensional systems 
theory, the FTM applies functional transformations in the time and space domain. This method can be summarized as follows:

- Apply the Laplace transformation to wave equation with respect to time. This removes the time derivatives and turns the inhomogeneous boundary-value problem into a boundary value problem for the space variable.

- Construct a suitable transformation for the space variable which removes the spatial derivatives and turns the boundary value problem into an algebraic equation. In this case, this is done through the use of a Sturm-Liouville transformation.

- To obtain a multidimensional transfer function, solve the algebraic equation for the transform of the solution of the partial differential equation.

From this multidimensional transfer function a discrete model in the form of a multidimensional difference equation can be derived which is suitable for computer implementation.

The main advantage of this method lies in its total lack of dispersion effects; unfortunately, the analytical solution of the Sturm-Liouville problem can be only find for simple geometries like rectangles or circles. This fact can limit its use for room acoustic. This problem can be solved using block-based models, which are a methodology for model complex physical models based into the interaction of several subsystems. This can be implemented interacting simple geometrical blocks implemented by pure FTM blocks [Petrausch and Rabenstein, 2005b] or different paradigms [Petrausch and Rabenstein, 2007], and create complex structures.

- Wave Digital Filters (WDF) is a particular kind of digital filter based on physical modeling principles [Fettweis, 1986]. It uses the bilinear transform to discretize the PDE of lumped elements such as resistors, capacitors and inductors (a deeper review of the mathematics behind WDFs is presented in Sec. 4.7). A WDF representation of a complete system is constructed by interconnecting simple discrete-time models of individual lumped components.

It is possible to use WDFs to integrate a PDE [Fettweis and Nitsche, 1991, Bilbao, 2004], and then it is possible to use this method in 
wave propagation simulation [Krauss and Rabenstein, 1995, Krauss et al., 1996, Schetelig and Rabenstein, 1998, Rabenstein and Schetelig, 1998]. In spite of their advantages, WDF have few known implementations in room acoustics, where the work of Kuntz and Rabenstein stands out [Kuntz and Rabenstein, 2006, 2008], who have implemented a room acoustic simulator based on a space-state representation of the WDF [Zayati, 1998, Zayati and Rabenstein, 2000].

- Upwind Leapfrog Schemes (ULS): This method is an alternative to the FDTD method used in order to reduce the inherent dispersion forcing to increase the computational cost to compute it. The Upwind Leapfrog Scheme or Linear Bicharacteristic Scheme [Thomas, 1996] is an economical alternative for the classical FDTD leapfrog scheme. The Upwind Leapfrog Scheme is a well-known scheme for unsteady aeroacoustic and electromagnetism applications, although in acoustics simulations it has not been frequently used. It has a more compact stencil compared with the classical leapfrog scheme. Clustering the stencil around the preferred directions (characteristics), it enables high accuracy with a low order of operations.

Although it has not been used in room acoustics, recently it has been proposed for Digital Sound Synthesis of string [Escolano and López, 2006a] and membranes instruments [Escolano and López, 2006b]. It is the personal opinion of the author that this method could be a good candidate for room acoustic simulation.

\subsection{Discussion}

In this chapter, an overview of the modeling techniques for room acoustic simulation has been presented. Since solving directly the particular inhomogeneous boundary value problem which represent the room under analysis is an extremely complex task, simplified models should be used to obtain approximated but accurate solutions.

In general, these methods are classified as geometrical and wave methods. Geometrical methods assume a sound propagation characteristics such as a ray and reflections completely specular; under these considerations, the geometrical methods work accurately for high frequencies, since many low frequency phenomena cannot be simulated, such as diffraction, occlusions 
and wave superpositions.

Wave methods solve numerically the wave equations, giving more accurate results than geometrical methods since the wave equations model all sound propagation phenomena, except air absorption. These methods are classified in two large groups according to the work domain: frequency and time-based. Wave methods in the frequency domain are suitable for room acoustic analysis, for a given frequency. This means they are suitable for steady-state simulations; but in cases where impulsive analysis is required, a simulation could be obtained frequency by frequency, increasing the computational cost severely, and finally the inverse Fourier transform might be used.

An alternative is the use of wave-based methods in the time domain or discrete-time methods: they are based on approaching the wave propagation through time and space discretization, creating a recursive algorithm which provides an acoustic variables distribution in all discretized space positions in every discrete time step. With these methods, impulsive analysis are obtained with an unique recursive algorithm, simplifying considerably the computational cost. The most popular discrete-time methods for room acoustic simulation are the finite-difference time-domain (FDTD) method and the Digital Waveguide Mesh (DWM) method. A survey of the suitability of discrete-time methods has shown how interesting results and advances are being carried out using these methods. 


\section{Discrete Time-based Methods for Room Acoustic Simulation}

AN INHOMOGENEOUS BOUNDARY VALUE PROBLEM allows us to mathematically describe the sound field in a room (see Chap. 2). If the partial differential equation that models the sound propagation is solved under specific conditions, an analytical solution of the entire sound field would be obtained. However, this can only be done in a few highly idealized cases, with reasonably moderate effort. The rooms which can be found in daily life are more or less irregular in shape, not only because of the walls, but also of furniture, columns, balconies and other wall irregularities. Therefore, alternative methods to the analytical solution have been developed to approach the solution.

As has been mentioned in Chap. 3, among the different proposed methods, the wave-based methods [Savioja, 1999] provide the most accurate solutions since they solve the wave equation numerically. Due to reasons explained in Sec. 3.4, time (or discrete-time) domain methods are most suitable for room acoustic simulation, since they allow broadband and impulsive signal analysis with just one simulation and involving a reasonable computational cost. 


\subsection{Introduction}

In this chapter, a review of these discrete-time methods used in room acoustic simulation is presented. Since this thesis is focused on some of them, it pays special attention to the Finite-Difference Time-Domain (FDTD) method and the Digital Waveguide Mesh (DWM), and a detailed overview of the mathematics lying behind these methods is presented.

It will be seen how from the basis of the sound propagation model analyzed in Chap. 2, it is possible to develop some algorithms able to simulate sound wave propagation in a particular scenario. It will also be shown how accurate the proposed methods are, since they consist of a numerical approach to the wave equation and no assumptions about wave behavior are made, as is the case of geometrical methods. Limitations of these methods are also presented, paying some attention to computational cost and the dispersion effects.

Furthermore, similarities and differences between the FDTD and the DWM methods are analyzed through the next pages, arriving at a hybrid method, which is one of the most used solutions for sound wave propagation simulation in room acoustics through wave-based methods.

This chapter is structured as follows: the first section deals with the Finite-Difference Time-Domain method. Next, mathematical concepts underlying the Digital Waveguide Mesh method are reviewed. After that, Sec. 4.4 demonstrates equivalence of these two methods in homogenous and inhomogeneous media; the differences between both models are also presented, arriving at hybrid models (Sec. 4.5). The next section shows how these methods are able to simulate low frequency properties of sound waves in the presence of obstacles, without any special modification of the algorithms. In Sec. 4.7, some basic aspects of the Wave Digital Filters (WDF) are introduced which, although they are not used as a sound simulation method itself in this thesis, they would play a basic role in Chap. 5. Finally, the chapter is summarized. 


\subsection{The Finite-Difference Time-Domain (FDTD) method}

The finite difference method is based upon an approximation of the definition of a mathematical derivative. For a given continuous function $f(x)$, its derivative is defined as

$$
\frac{d f(x)}{d x}=\lim _{\Delta x \rightarrow 0} \frac{f(x+\Delta x)-f(x)}{(x+\Delta x)-x}
$$

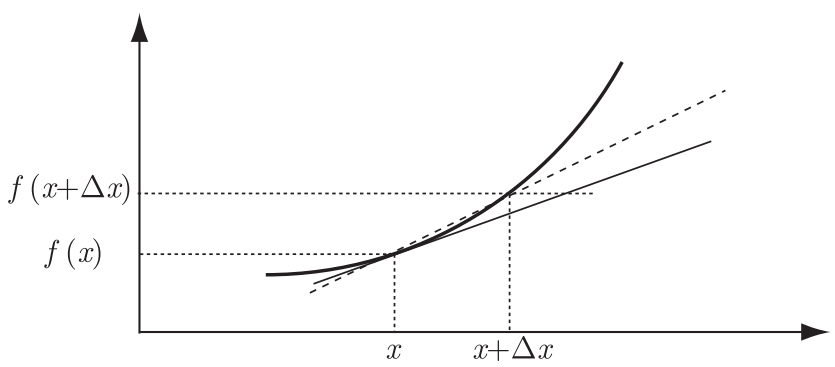

Figure 4.1. The definition of the derivative, $d f(x) / d x$, of the function $f(x)$

Equation 4.1 becomes exact when $\Delta x$ tends to zero. However, in finite calculations, the increment $\Delta x$ cannot tend to zero and an approximation should be done. Then, the next equality is assumed

$$
\frac{d f(x)}{d x}=\frac{f(x+\Delta x)-f(x)}{\Delta x}+\varepsilon(\Delta x),
$$

where $\varepsilon(\Delta x)$ is a shorthand notation for the reminder term, which tends to zero as the increment.

The particular approach of Eq. 4.2 is known as forward difference formula [Jordan, 1950]. Also, there exist other finite difference approaches, such as backward difference formula (Eq. 4.3) and central difference formula (Eq. 4.4) 


$$
\begin{aligned}
\frac{d f(x)}{d x} & =\frac{f(x)-f(x-\Delta x)}{\Delta x}+\varepsilon(\Delta x), \\
\frac{d f(x)}{d x} & =\frac{f(x+\Delta x)-f(x-\Delta x)}{2 \Delta x}+\varepsilon\left(\Delta x^{2}\right) .
\end{aligned}
$$

It has to be noted how the central approach of the derivatives has an error term $\varepsilon\left(\Delta x^{2}\right)$ which tends to zero faster than the forward and backward approach; then, the approach of Eq. 4.4 is more accurate than the others.

By applying the central difference approach twice, it is possible to arrive at the central difference approach of the second derivative

$$
\frac{d^{2} f(x)}{d x^{2}}=\frac{f(x+\Delta x)-2 f(x)+f(x-\Delta x)}{\Delta x^{2}}+\varepsilon\left(\Delta x^{2}\right) .
$$

The finite difference method consists on substituting the derivative of continuous differential equations by the finite difference approaches without the error term $\varepsilon$.

In the case of PDEs, the partial derivatives follow the same scheme for the multidimensional functions. Let us consider the function $f(x, t)$, the first and second derivative for each one of their variables are

$$
\begin{aligned}
\frac{\partial f(x, t)}{\partial x} & \simeq \frac{f(x+\Delta x, t)-f(x-\Delta x, t)}{2 \Delta x} \\
\frac{\partial f(x, t)}{\partial t} & \simeq \frac{f(x, t+\Delta t)-f(x, t-\Delta t)}{2 \Delta t} \\
\frac{\partial^{2} f(x, t)}{\partial x^{2}} & \simeq \frac{f(x+\Delta x, t)-2 f(x, t)+f(x-\Delta x, t)}{\Delta x^{2}} \\
\frac{\partial^{2} f(x, t)}{\partial t^{2}} & \simeq \frac{f(x, t+\Delta t)-2 f(x, t)+f(x, t-\Delta t)}{\Delta t^{2}}
\end{aligned}
$$

Based on the introduced finite differences approach, the standard FDTD is defined as a second order central finite difference approximation to the derivatives involved in the wave equations. The sound field variables, pressure and particle velocity components, are staggered, following the electromagnetic case in the so-called Yee's cell [Taflove, 1995]. In the acoustic 
case, the pressure is situated in the center and each one of the particle velocity components is situated in the external face of the cell, according to Fig. 4.2.

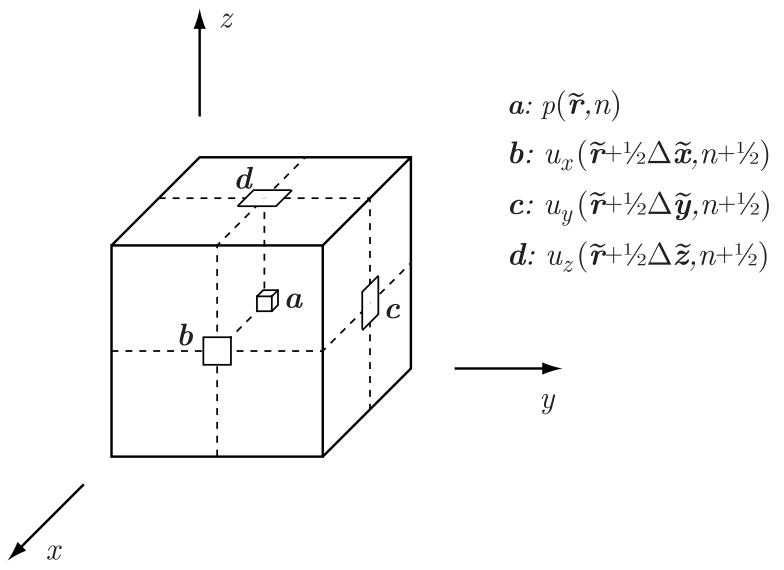

Figure 4.2. Yee's cell for the acoustic FDTD algorithm.

In the original algorithm [Botteldooren, 1995], the Euler (Eq. 2.31) and conservation of mass equation (Eq. 2.30) are the equations used. Now, applying the above ideas, the finite difference approach of the linearized homogeneous Euler equation (Eq. 2.31) is

$$
\begin{aligned}
\rho_{0} \frac{u_{x}\left(\tilde{\mathbf{r}}-\frac{1}{2} \Delta \tilde{\mathbf{x}}, n+\frac{1}{2}\right)-u_{x}\left(\tilde{\mathbf{r}}-\frac{1}{2} \Delta \tilde{\mathbf{x}}, n-\frac{1}{2}\right)}{\Delta t} & =-\frac{p(\tilde{\mathbf{r}}, n)-p(\tilde{\mathbf{r}}-\Delta \tilde{\mathbf{x}}, n)}{\Delta x}, \\
\rho_{0} \frac{u_{y}\left(\tilde{\mathbf{r}}-\frac{1}{2} \Delta \tilde{\mathbf{y}}, n+\frac{1}{2}\right)-u_{y}\left(\tilde{\mathbf{r}}-\frac{1}{2} \Delta \tilde{\mathbf{y}}, n-\frac{1}{2}\right)}{\Delta t} & =-\frac{p(\tilde{\mathbf{r}}, n)-p(\tilde{\mathbf{r}}-\Delta \tilde{\mathbf{y}}, n)}{\Delta y} \\
\rho_{0} \frac{u_{z}\left(\tilde{\mathbf{r}}-\frac{1}{2} \Delta \tilde{\mathbf{z}}, n+\frac{1}{2}\right)-u_{z}\left(\tilde{\mathbf{r}}-\frac{1}{2} \Delta \tilde{\mathbf{z}}, n-\frac{1}{2}\right)}{\Delta t} & =-\frac{p(\tilde{\mathbf{r}}, n)-p(\tilde{\mathbf{r}}-\Delta \tilde{\mathbf{z}}, n)}{\Delta z}
\end{aligned}
$$

whereas the linearized homogeneous conservation of the mass equation (Eq. 2.30) is 


$$
\begin{aligned}
\frac{p(\tilde{\mathbf{r}}, n+1)-p(\tilde{\mathbf{r}}, n)}{\Delta t}= & -\rho_{0} c^{2} \frac{u_{x}\left(\tilde{\mathbf{r}}+\frac{1}{2} \Delta \tilde{\mathbf{x}}, n+\frac{1}{2}\right)-u_{x}\left(\tilde{\mathbf{r}}-\frac{1}{2} \Delta \tilde{\mathbf{x}}, n+\frac{1}{2}\right)}{\Delta x} \\
& -\rho_{0} c^{2} \frac{u_{y}\left(\tilde{\mathbf{r}}+\frac{1}{2} \Delta \tilde{\mathbf{y}}, n+\frac{1}{2}\right)-u_{y}\left(\tilde{\mathbf{r}}-\frac{1}{2} \Delta \tilde{\mathbf{y}}, n+\frac{1}{2}\right)}{\Delta y} \\
& -\rho_{0} c^{2} \frac{u_{z}\left(\tilde{\mathbf{r}}+\frac{1}{2} \Delta \tilde{\mathbf{z}}, n+\frac{1}{2}\right)-u_{z}\left(\tilde{\mathbf{r}}-\frac{1}{2} \Delta \tilde{\mathbf{z}}, n+\frac{1}{2}\right)}{\Delta z},
\end{aligned}
$$

where the notation $p(\mathbf{r}, t)=p(i \Delta x, j \Delta y, k \Delta z, n \Delta t)=p(\tilde{\mathbf{r}}, n)$ is used. Furthermore, in the following, the notation $\Delta \tilde{\mathbf{x}}=(\Delta x, 0,0), \Delta \tilde{\mathbf{y}}=(0, \Delta y, 0)$ and $\Delta \tilde{\mathbf{z}}=(0,0, \Delta z)$ is used as well.

After some algebraic manipulations of these equations, the acoustic FDTD method is defined

$$
\begin{aligned}
u_{x}\left(\tilde{\mathbf{r}}-\frac{1}{2} \Delta \tilde{\mathbf{x}}, n+\frac{1}{2}\right) & =u_{x}\left(\tilde{\mathbf{r}}-\frac{1}{2} \Delta \tilde{\mathbf{x}}, n-\frac{1}{2}\right) \\
& -\frac{\Delta t}{\rho_{0} \Delta x}(p(\tilde{\mathbf{r}}, n)-p(\tilde{\mathbf{r}}-\Delta \tilde{\mathbf{x}}, n)) \\
u_{y}\left(\tilde{\mathbf{r}}-\frac{1}{2} \Delta \tilde{\mathbf{y}}, n+\frac{1}{2}\right) & =u_{y}\left(\tilde{\mathbf{r}}-\frac{1}{2} \Delta \tilde{\mathbf{y}}, n-\frac{1}{2}\right) \\
& -\frac{\Delta t}{\rho_{0} \Delta y}(p(\tilde{\mathbf{r}}, n)-p(\tilde{\mathbf{r}}-\Delta \tilde{\mathbf{y}}, n)), \\
u_{z}\left(\tilde{\mathbf{r}}-\frac{1}{2} \Delta \tilde{\mathbf{z}}, n+\frac{1}{2}\right) & =u_{z}\left(\tilde{\mathbf{r}}-\frac{1}{2} \Delta \tilde{\mathbf{z}}, n-\frac{1}{2}\right) \\
& -\frac{\Delta t}{\rho_{0} \Delta z}(p(\tilde{\mathbf{r}}, n)-p(\tilde{\mathbf{r}}-\Delta \tilde{\mathbf{z}}, n)), \\
p(\tilde{\mathbf{r}}, n+1) & =\frac{\rho_{0} c^{2} \Delta t}{\Delta x}\left(u_{x}\left(\tilde{\mathbf{r}}+\frac{1}{2} \Delta \tilde{\mathbf{x}}, n+\frac{1}{2}\right)-u_{x}\left(\tilde{\mathbf{r}}-\frac{1}{2} \Delta \tilde{\mathbf{x}}, n+\frac{1}{2}\right)\right) \\
& -\frac{\rho_{0} c^{2} \Delta t}{\Delta y}\left(u_{y}\left(\tilde{\mathbf{r}}+\frac{1}{2} \Delta \tilde{\mathbf{y}}, n+\frac{1}{2}\right)-u_{y}\left(\tilde{\mathbf{r}}-\frac{1}{2} \Delta \tilde{\mathbf{y}}, n+\frac{1}{2}\right)\right) \\
& -\frac{\rho_{0} c^{2} \Delta t}{\Delta z}\left(u_{z}\left(\tilde{\mathbf{r}}+\frac{1}{2} \Delta \tilde{\mathbf{z}}, n+\frac{1}{2}\right)-u_{z}\left(\tilde{\mathbf{r}}-\frac{1}{2} \Delta \tilde{\mathbf{z}}, n+\frac{1}{2}\right)\right) .
\end{aligned}
$$

These equations are updated in time by using a leap-frog scheme. First, $u$ 's at time level $n+1 / 2$ are computed from $p$ 's at time level $n$ and previous $u$ 's at time level $n-1 / 2$. Then, $p$ 's at time level $n+1$ are computed from $u$ 's at time level $n+1 / 2$ and previous $p$ 's at time level $n$. This process repeats itself until the temporal simulation is completed. Note that the leapfrog 

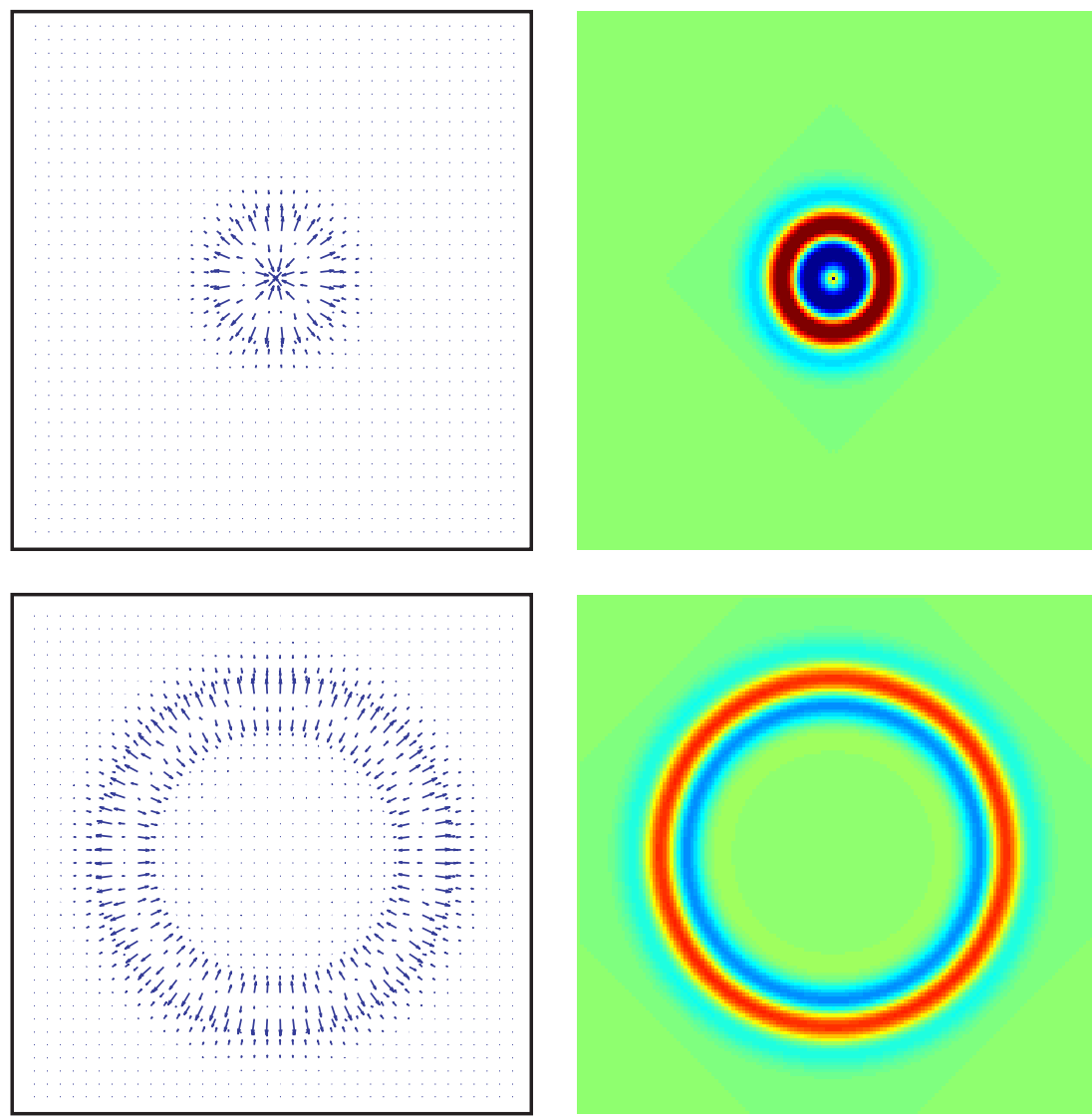

Figure 4.3. Particle velocity and pressure propagation of a gaussian source simulated by the FDTD method.

scheme does not introduce dissipation and is reversible in time because of its central symmetry in both time and space. It may happen that a scheme built from symmetric and reversible units may lose any appearance of symmetry, but Roe has shown that such a scheme is nevertheless reversible [Roe, 1998]. Figure 4.3 presents a broadband gaussian source propagation which has been simulated by using the FDTD method and the pressure and the particle velocity vector are drawn at different times.

When a finite difference scheme is used substituting the derivatives on an ODE or PDE, it is mandatory to demonstrate its: 
- Consistency: a finite difference scheme tends to the differential problem when the increments decrease.

- Convergence: a finite difference approximation converges, when the increments decrease, to the original differential equation solution.

- Stability: bounded variations in the initial conditions produce bounded variations in the solution.

All these properties can be demonstrated through the LaxÜ-Richtmyer equivalence theorem [Strikwerda, 1989]. Although the scope of this thesis is not to demonstrate each one of these points for the FDTD method, some comments about the dispersion and stability are presented in the next sections since they will be addressed many times in the next chapters.

\subsubsection{Grid Dispersion}

With any discretized solution to wave type equations, one should be concerned about grid dispersion. Grid dispersion is the fact that waves in a numerical grid travel at speeds slightly different than in a real space [Maloney and Cummings, 1995]. Furthermore, this grid dispersion tends to be frequency and angle dependent. Therefore, it is difficult to calibrate for, and the best strategy is to try to minimize the amount of grid dispersion. To solve the dispersion relation, a general plane wave propagating throughout the grid is assumed.

$$
p, u_{x}, u_{y}, u_{z} \propto e^{j\left(\omega t-k_{x} x-k_{y} y-k_{z} z\right)}
$$

where $k_{x}, k_{y}$ and $k_{z}$ are the components of the numerical grid wavenumber $k$. Using Eq. 4.18 to reduce Eqs. 4.14-4.17 expressions to: 


$$
\begin{aligned}
{\left[1-e^{-j \omega \Delta t}\right] u_{x}\left(\tilde{\mathbf{r}}-\frac{1}{2} \Delta \tilde{\mathbf{x}}, n+\frac{1}{2}\right)=} & -\frac{\Delta t}{\rho_{0} \Delta x}\left[1-e^{-j k_{x} \Delta x}\right] p(\tilde{\mathbf{r}}, n), \\
{\left[1-e^{-j \omega \Delta t}\right] u_{y}\left(\tilde{\mathbf{r}}-\frac{1}{2} \Delta \tilde{\mathbf{y}}, n+\frac{1}{2}\right)=} & -\frac{\Delta t}{\rho_{0} \Delta y}\left[1-e^{-j k_{y} \Delta y}\right] p(\tilde{\mathbf{r}}, n), \\
{\left[1-e^{-j \omega \Delta t}\right] u_{z}\left(\tilde{\mathbf{r}}-\frac{1}{2} \Delta \tilde{\mathbf{z}}, n+\frac{1}{2}\right)=} & -\frac{\Delta t}{\rho_{0} \Delta z}\left[1-e^{-j k_{z} \Delta z}\right] p(\tilde{\mathbf{r}}, n), \\
{\left[e^{j \omega \Delta t}-1\right] p(\tilde{\mathbf{r}}, n)=} & -\rho_{0} c^{2} \frac{\Delta t}{\Delta x}\left[e^{j k_{x} \Delta x}-1\right] u_{x}\left(\tilde{\mathbf{r}}-\frac{1}{2} \Delta \tilde{\mathbf{x}}, n+\frac{1}{2}\right) \\
& -\rho_{0} c^{2} \frac{\Delta t}{\Delta y}\left[e^{j k_{y} \Delta y}-1\right] u_{y}\left(\tilde{\mathbf{r}}-\frac{1}{2} \Delta \tilde{\mathbf{y}}, n+\frac{1}{2}\right) \\
& -\rho_{0} c^{2} \frac{\Delta t}{\Delta z}\left[e^{j k_{z} \Delta z}-1\right] u_{z}\left(\tilde{\mathbf{r}}-\frac{1}{2} \Delta \tilde{\mathbf{z}}, n+\frac{1}{2}\right) .
\end{aligned}
$$

Then, substituting Eqs. 4.19-4.21 into Eq. 4.22 yields after some algebraic manipulation

$$
\begin{aligned}
& {\left[\sin ^{2}(\omega \Delta t)-\left(\frac{c \Delta t}{\Delta x}\right)^{2} \sin ^{2}\left(\frac{k_{x} \Delta x}{2}\right)\right.} \\
& \left.-\left(\frac{c \Delta t}{\Delta y}\right)^{2} \sin ^{2}\left(\frac{k_{y} \Delta y}{2}\right)-\left(\frac{c \Delta t}{\Delta z}\right)^{2} \sin ^{2}\left(\frac{k_{z} \Delta z}{2}\right)\right] p(\tilde{\mathbf{r}}, n)=0 .
\end{aligned}
$$

In general, $p(\tilde{\mathbf{r}}, n) \neq 0$ and thus

$$
\begin{aligned}
\sin ^{2}(\omega \Delta t) & =\left(\frac{c \Delta t}{\Delta x}\right)^{2} \sin ^{2}\left(\frac{k_{x} \Delta x}{2}\right) \\
& +\left(\frac{c \Delta t}{\Delta y}\right)^{2} \sin ^{2}\left(\frac{k_{y} \Delta y}{2}\right)+\left(\frac{c \Delta t}{\Delta z}\right)^{2} \sin ^{2}\left(\frac{k_{z} \Delta z}{2}\right)
\end{aligned}
$$

This is the dispersion relation for the discretized solution; it is identical to the dispersion relation for the FDTD technique applied to Maxwell's equation. For a uniform grid $(\Delta s=\Delta x=\Delta y=\Delta z)$, is commonly rewritten in the form 


$$
\begin{aligned}
\sin ^{2}\left(\frac{\pi}{2} \frac{\Delta s}{\lambda_{0}}\right) & =\left(\frac{c \Delta t}{\Delta s}\right)^{2} \sin ^{2}\left(\pi \cos \alpha \frac{\Delta s}{\lambda_{n}}\right)+\left(\frac{c \Delta t}{\Delta s}\right)^{2} \sin ^{2}\left(\pi \cos \beta \frac{\Delta s}{\lambda_{n}}\right) \\
& +\left(\frac{c \Delta t}{\Delta s}\right)^{2} \sin ^{2}\left(\pi \cos \gamma \frac{\Delta s}{\lambda_{n}}\right)
\end{aligned}
$$

where $\lambda_{0}$ is the wavelength in real space, $\lambda_{n}$ is the numerical wavelength, and $\cos \alpha, \cos \beta$ and $\cos \gamma$ are the direction cosines of the propagating plane wave.

Dispersion is one of the most important handicaps of the FDTD method and through the literature, several solutions and/or improvements have been proposed, such as [Wagner and Schneider, 2005]; however, to deal with this particularity of the method is out of the scope of this thesis. On the other hand, a complete framework of this topic can be found in [Tam and Webb, 1992].

A compromise solution for presenting results with a reduced dispersion is to limit maximum frequency of analysis up to a normalized frequency of $f_{\max }=0.25 f_{s}$ [Duyne and Smith, 1993]. In the following, results will be presented using a normalized frequency scale.

\subsubsection{Stability}

Another important criteria is the stability of the algorithm. The stability relation is derived in a similar way. Again, a general plane wave propagated throughout the grid is assumed

$$
p, u_{x}, u_{y}, u_{z} \propto \xi e^{-j\left(k_{x} x+k_{y} y+k_{z} z\right)} .
$$

Substituting Eq. 4.26 in Eqs. 4.14-4.17, system equation formed by Eqs. 4.27-4.30 is obtained 


$$
\begin{aligned}
{\left[1-\xi^{-1}\right] u_{x}\left(\tilde{\mathbf{r}}-\frac{1}{2} \Delta \tilde{\mathbf{x}}, n+\frac{1}{2}\right) } & -\frac{\Delta t}{\rho_{0} \Delta x}\left[1-e^{-j k_{x} \Delta x}\right] p(\tilde{\mathbf{r}}, n), \\
{\left[1-\xi^{-1}\right] u_{y}\left(\tilde{\mathbf{r}}-\frac{1}{2} \Delta \tilde{\mathbf{y}}, n+\frac{1}{2}\right) } & =-\frac{\Delta t}{\rho_{0} \Delta y}\left[1-e^{-j k_{y} \Delta y}\right] p(\tilde{\mathbf{r}}, n), \\
{\left[1-\xi^{-1}\right] u_{z}\left(\tilde{\mathbf{r}}-\frac{1}{2} \Delta \tilde{\mathbf{z}}, n+\frac{1}{2}\right) } & =-\frac{\Delta t}{\rho_{0} \Delta z}\left[1-e^{-j k_{z} \Delta z}\right] p(\tilde{\mathbf{r}}, n), \\
{[\xi-1] p(\tilde{\mathbf{r}}, n) } & =-\rho_{0} c^{2} \frac{\Delta t}{\Delta x}\left[e^{j k_{x} \Delta x}-1\right] u_{x}\left(\tilde{\mathbf{r}}-\frac{1}{2} \Delta \tilde{\mathbf{x}}, n+\frac{1}{2}\right) \\
& -\rho_{0} c^{2} \frac{\Delta t}{\Delta y}\left[e^{j k_{y} \Delta y}-1\right] u_{y}\left(\tilde{\mathbf{r}}-\frac{1}{2} \Delta \tilde{\mathbf{y}}, n+\frac{1}{2}\right) \\
& -\rho_{0} c^{2} \frac{\Delta t}{\Delta z}\left[e^{j k_{z} \Delta z}-1\right] u_{z}\left(\tilde{\mathbf{r}}-\frac{1}{2} \Delta \tilde{\mathbf{z}}, n+\frac{1}{2}\right)
\end{aligned}
$$

Again, substituting Eqs. 4.27-4.29 into Eq. 4.30 yields after some manipulation

$$
\xi^{2}-2 A \xi+1=0
$$

where

$$
\begin{aligned}
A=1-2 & \left(\frac{c \Delta t}{\Delta x}\right)^{2} \sin ^{2}\left(\frac{k_{x} \Delta x}{2}\right) \\
& -2\left(\frac{c \Delta t}{\Delta y}\right)^{2} \sin ^{2}\left(\frac{k_{y} \Delta y}{2}\right)-2\left(\frac{c \Delta t}{\Delta z}\right)^{2} \sin ^{2}\left(\frac{k_{z} \Delta z}{2}\right) .
\end{aligned}
$$

The solution of Eq. 4.31 is

$$
\xi=A \pm \sqrt{A^{2}-1}
$$

An unstable solution will occur if $|\xi|>1$. This only occurs when $|A|>1$; thus

$$
c \Delta t>\frac{1}{\sqrt{\left(\frac{1}{\Delta x}\right)^{2}+\left(\frac{1}{\Delta y}\right)^{2}+\left(\frac{1}{\Delta z}\right)^{2}}}
$$


However, when $|A| \leq 1$, then $|\xi|=1$ and the grid is stable; thus

$$
c \Delta t \leq \frac{1}{\sqrt{\left(\frac{1}{\Delta x}\right)^{2}+\left(\frac{1}{\Delta y}\right)^{2}+\left(\frac{1}{\Delta z}\right)^{2}}}
$$

This condition is known as the Courant-Friedrichs-Lewy condition [Courant et al., 1928], or Courant condition for short, and it determines the relation between the temporal and spatial increment to assure the stability of the algorithm. For practical considerations, the condition is fixed as an equality, and considering homogeneous meshes $(\Delta x=\Delta y=\Delta z)$, the stability condition becomes

$$
\Delta x=\sqrt{N} c \Delta t
$$

where $N$ is the dimension of the mesh.

\subsubsection{The FDTD method in a homogeneous medium}

Although the FDTD method is considered an efficient method, it is true that the memory and computational requirements are still high. It must be considered that for each discretized point (and for each time step) in a $3 \mathrm{D}$ simulation, four variables must be stored. In applications such as room acoustic simulation, the particle velocity is not necessarily computed since human hearing is only sensitive to pressure changes. This implication helps to build a more efficient method.

This modification is obtained using the wave equation in an homogeneous medium ${ }^{1}$. As detailed in Sec. 2.3.1, this equation is obtained when the mass conservation and Euler equations (Eq. 2.30 and 2.31) are combined in order to give a pressure-dependent equation (Eq. 2.32).

Using a central finite difference scheme for second order derivatives (Eq. 4.5) and assuming Courant stability condition (Eq. 4.36), $\Delta x=\sqrt{3} c \Delta t$, the FDTD method is simplified to

\footnotetext{
${ }^{1}$ One has to try to not confuse the homogeneous wave equation (no source term is included) for wave equation in a homogeneous medium (medium properties, i.e., speed of sound and air density, are constant).
} 


$$
\begin{aligned}
p(\tilde{\mathbf{r}}, n+1) & =\frac{1}{3}[p(\tilde{\mathbf{r}}+\Delta \tilde{\mathbf{x}}, n)+p(\tilde{\mathbf{r}}-\Delta \tilde{\mathbf{x}}, n)+p(\tilde{\mathbf{r}}+\Delta \tilde{\mathbf{y}}, n) \\
& +p(\tilde{\mathbf{r}}-\Delta \tilde{\mathbf{y}}, n)+p(\tilde{\mathbf{r}}+\Delta \tilde{\mathbf{z}}, n)+p(\tilde{\mathbf{r}}-\Delta \tilde{\mathbf{z}}, n)]-p(\tilde{\mathbf{r}}, n-1)
\end{aligned}
$$

Savioja et al. proposed this version of the FDTD method, which is more appropriate for its use in room acoustics simulation [Savioja et al., 1994]. Aspects such as stability and dispersion remain the same as in the original FDTD method. If particle velocity is necessary, it can be obtained from the local use of the discretized Euler equation (Eq. 4.14). In this thesis, this version will be indicated as Savioja's approch, in order to be differentiated from the Botteldooren's approach (see Sec. 4.2).

From the inhomogeneous wave equation (including source term $s(\mathbf{r}, t)$ ), the finite difference approach becomes

$$
\begin{aligned}
p(\tilde{\mathbf{r}}, n+1) & =\frac{1}{3}[p(\tilde{\mathbf{r}}+\Delta \tilde{\mathbf{x}}, n)+p(\tilde{\mathbf{r}}-\Delta \tilde{\mathbf{x}}, n)+p(\tilde{\mathbf{r}}+\Delta \tilde{\mathbf{y}}, n) \\
& +p(\tilde{\mathbf{r}}-\Delta \tilde{\mathbf{y}}, n)+p(\tilde{\mathbf{r}}+\Delta \tilde{\mathbf{z}}, n)+p(\tilde{\mathbf{r}}-\Delta \tilde{\mathbf{z}}, n)]-p(\tilde{\mathbf{r}}, n-1) \\
& +3 c^{2} \Delta t^{2} s(\tilde{\mathbf{r}}, n) .
\end{aligned}
$$

\subsubsection{The FDTD method in an inhomogeneous medium}

In this subsection, the FDTD method is obtained for an inhomogeneous media, for reasons which will arise in Sec. 4.4. This inhomogeneity in a medium is related to a variable density as a function of the position.

Let us consider the linearized conservation of mass and Euler equations with a variable density

$$
\begin{aligned}
\frac{\partial p(\mathbf{r}, t)}{\partial t}+\rho_{0}(\mathbf{r}) c^{2} \nabla \cdot \mathbf{u}(\mathbf{r}, t) & =0 \\
\rho_{0}(\mathbf{r}) \frac{\partial \mathbf{u}(\mathbf{r}, t)}{\partial t}+\nabla p(\mathbf{r}, t) & =0 .
\end{aligned}
$$

The single partial differential equation for the acoustic part of the pressure results when one takes the time derivative of Eq. 4.39 and inserts it into the divergence of Eq. 4.40. In this way, the time derivative of the 
particle velocity is expressed in terms of the pressure. The resulting equation [Bergmann, 1946] is

$$
\nabla \cdot\left(\frac{1}{\rho_{0}(\mathbf{r})} \nabla p(\mathbf{r}, t)\right)-\frac{1}{\rho_{0}(\mathbf{r}) c^{2}} \frac{\partial^{2} p(\mathbf{r}, t)}{\partial t^{2}}=0
$$

It should be noted that if the ambient density is independent of position, the equation is reduced to the wave equation of linear acoustics. For the purpose of this thesis, the finite-difference approximation of the spatial derivatives proposed by Boore [Boore, 1972] or by Kelly [Kelly et al., 1976] is used. However, in order to avoid using long equations, following mathematical developments are presented for a 2-D mesh

$$
\begin{aligned}
& {\left[\frac{\partial}{\partial x}\left(\frac{1}{\rho_{0}(\mathbf{r})} \nabla p(\mathbf{r}, t)\right)\right]_{\mathbf{r}=\tilde{\mathbf{r}}} \approx} \\
& \approx \frac{1}{\Delta x^{2}}\left(\frac{p(\tilde{\mathbf{r}}+\Delta \tilde{\mathbf{x}}, n)-p(\tilde{\mathbf{r}}, n)}{\rho_{0}\left(\tilde{\mathbf{r}}+\frac{1}{2} \Delta \tilde{\mathbf{x}}\right)}-\frac{p(\tilde{\mathbf{r}}, n)-p(\tilde{\mathbf{r}}-\Delta \tilde{\mathbf{x}}, n)}{\rho_{0}\left(\tilde{\mathbf{r}}-\frac{1}{2} \Delta \tilde{\mathbf{x}}\right)}\right), \\
& {\left[\frac{\partial}{\partial y}\left(\frac{1}{\rho_{0}(\mathbf{r})} \nabla p(\mathbf{r}, t)\right)\right]_{\mathbf{r}=\tilde{\mathbf{r}}} \approx} \\
& \approx \frac{1}{\Delta y^{2}}\left(\frac{p(\tilde{\mathbf{r}}+\Delta \tilde{\mathbf{y}}, n)-p(\tilde{\mathbf{r}}, n)}{\rho_{0}\left(\tilde{\mathbf{r}}+\frac{1}{2} \Delta \tilde{\mathbf{y}}\right)}-\frac{p(\tilde{\mathbf{r}}, n)-p(\tilde{\mathbf{r}}-\Delta \tilde{\mathbf{y}}, n)}{\rho_{0}\left(\tilde{\mathbf{r}}-\frac{1}{2} \Delta \tilde{\mathbf{y}}\right)}\right),
\end{aligned}
$$

where

$$
\begin{aligned}
& \frac{1}{\rho_{0}\left(\tilde{\mathbf{r}}+\frac{1}{2} \Delta \tilde{\mathbf{x}}\right)}=\frac{1}{2}\left(\frac{1}{\rho_{0}(\tilde{\mathbf{r}}+\Delta \tilde{\mathbf{x}})}+\frac{1}{\rho_{0}(\tilde{\mathbf{r}})}\right), \\
& \frac{1}{\rho_{0}\left(\tilde{\mathbf{r}}-\frac{1}{2} \Delta \tilde{\mathbf{x}}\right)}=\frac{1}{2}\left(\frac{1}{\rho_{0}(\tilde{\mathbf{r}})}+\frac{1}{\rho_{0}(\tilde{\mathbf{r}}-\Delta \tilde{\mathbf{x}})}\right), \\
& \frac{1}{\rho_{0}\left(\tilde{\mathbf{r}}+\frac{1}{2} \Delta \tilde{\mathbf{y}}\right)}=\frac{1}{2}\left(\frac{1}{\rho_{0}(\tilde{\mathbf{r}}+\Delta \tilde{\mathbf{y}})}+\frac{1}{\rho_{0}(\tilde{\mathbf{r}})}\right), \\
& \frac{1}{\rho_{0}\left(\tilde{\mathbf{r}}-\frac{1}{2} \Delta \tilde{\mathbf{y}}\right)}=\frac{1}{2}\left(\frac{1}{\rho_{0}(\tilde{\mathbf{r}})}+\frac{1}{\rho_{0}(\tilde{\mathbf{r}}-\Delta \tilde{\mathbf{y}})}\right) .
\end{aligned}
$$

In the same way, the ambient density could be defined at a point $\tilde{\mathbf{r}}$ as 


$$
\frac{1}{\rho_{0}(\tilde{\mathbf{r}})}=\frac{1}{4}\left(\frac{1}{\rho_{0}\left(\tilde{\mathbf{r}}+\frac{1}{2} \Delta \tilde{\mathbf{x}}\right)}+\frac{1}{\rho_{0}\left(\tilde{\mathbf{r}}-\frac{1}{2} \Delta \tilde{\mathbf{x}}\right)}+\frac{1}{\rho_{0}\left(\tilde{\mathbf{r}}+\frac{1}{2} \Delta \tilde{\mathbf{y}}\right)}+\frac{1}{\rho_{0}\left(\tilde{\mathbf{r}}-\frac{1}{2} \Delta \tilde{\mathbf{y}}\right)}\right) .
$$

In accordance with the finite-differences approach of the spatial derivatives, Eq. 4.42, and the standard time derivative approach used during this work, the finite-difference approximation of the heterogeneous wave equation can be written as

$$
\begin{aligned}
& \frac{p(\tilde{\mathbf{r}}+\Delta \tilde{\mathbf{x}}, n)}{\rho_{0}\left(\tilde{\mathbf{r}}+\frac{1}{2} \Delta \tilde{\mathbf{x}}\right)}+\frac{p(\tilde{\mathbf{r}}-\Delta \tilde{\mathbf{x}}, n)}{\rho_{0}\left(\tilde{\mathbf{r}}-\frac{1}{2} \Delta \tilde{\mathbf{x}}\right)}+\frac{p(\tilde{\mathbf{r}}+\Delta \tilde{\mathbf{y}}, n)}{\rho_{0}\left(\tilde{\mathbf{r}}+\frac{1}{2} \Delta \tilde{\mathbf{y}}\right)}+\frac{p(\tilde{\mathbf{r}}-\Delta \tilde{\mathbf{y}}, n)}{\rho_{0}\left(\tilde{\mathbf{r}}-\frac{1}{2} \Delta \tilde{\mathbf{y}}\right)} \\
- & p(\tilde{\mathbf{r}}, n)\left(\frac{1}{\rho_{0}\left(\tilde{\mathbf{r}}+\frac{1}{2} \Delta \tilde{\mathbf{x}}\right)}+\frac{1}{\rho_{0}\left(\tilde{\mathbf{r}}-\frac{1}{2} \Delta \tilde{\mathbf{x}}\right)}+\frac{1}{\rho_{0}\left(\tilde{\mathbf{r}}+\frac{1}{2} \Delta \tilde{\mathbf{y}}\right)}+\frac{1}{\rho_{0}\left(\tilde{\mathbf{r}}-\frac{1}{2} \Delta \tilde{\mathbf{y}}\right)}\right) \\
- & \frac{2}{\rho_{0}(\tilde{\mathbf{r}})}(p(\tilde{\mathbf{r}}, n+1)-2 p(\tilde{\mathbf{r}}, n)+p(\tilde{\mathbf{r}}, n-1))=0,
\end{aligned}
$$

where the Courant formula has been assumed following Eq. 4.36.

In accordance with Eq. 4.47, Eq. 4.48 is reduced to the form

$$
\begin{aligned}
\frac{p(\tilde{\mathbf{r}}+\Delta \tilde{\mathbf{x}}, n)}{\rho_{0}\left(\tilde{\mathbf{r}}+\frac{1}{2} \Delta \tilde{\mathbf{x}}\right)} & +\frac{p(\tilde{\mathbf{r}}-\Delta \tilde{\mathbf{x}}, n)}{\rho_{0}\left(\tilde{\mathbf{r}}-\frac{1}{2} \Delta \tilde{\mathbf{x}}\right)}+\frac{p(\tilde{\mathbf{r}}+\Delta \tilde{\mathbf{y}}, n)}{\rho_{0}\left(\tilde{\mathbf{r}}+\frac{1}{2} \Delta \tilde{\mathbf{y}}\right)}+\frac{p(\tilde{\mathbf{r}}-\Delta \tilde{\mathbf{y}}, n)}{\rho_{0}\left(\tilde{\mathbf{r}}-\frac{1}{2} \Delta \tilde{\mathbf{y}}\right)} \\
& -\frac{2}{\rho_{0}(\tilde{\mathbf{r}})}(p(\tilde{\mathbf{r}}, n+1)+p(\tilde{\mathbf{r}}, n-1))=0
\end{aligned}
$$

and solving $p(\tilde{\mathbf{r}}, n+1)$, it leads to

$$
\begin{aligned}
p(\tilde{\mathbf{r}}, n+1) & =\frac{2\left(\frac{p(\tilde{\mathbf{r}}+\Delta \tilde{\mathbf{x}}, n)}{\rho_{0}\left(\tilde{\mathbf{r}}+\frac{1}{2} \Delta \tilde{\mathbf{x}}\right)}+\frac{p(\tilde{\mathbf{r}}-\Delta \tilde{\mathbf{x}}, n)}{\rho_{0}\left(\tilde{\mathbf{r}}-\frac{1}{2} \Delta \tilde{\mathbf{x}}\right)}+\frac{p(\tilde{\mathbf{r}}+\Delta \tilde{\mathbf{y}}, n)}{\rho_{0}\left(\tilde{\mathbf{r}}+\frac{1}{2} \Delta \tilde{\mathbf{y}}\right)}+\frac{p(\tilde{\mathbf{r}}-\Delta \tilde{\mathbf{y}}, n)}{\rho_{0}\left(\tilde{\mathbf{r}}-\frac{1}{2} \Delta \tilde{\mathbf{y}}\right)}\right)}{\frac{1}{\rho_{0}\left(\tilde{\mathbf{r}}+\frac{1}{2} \Delta \tilde{\mathbf{x}}\right)}+\frac{1}{\rho_{0}\left(\tilde{\mathbf{r}}-\frac{1}{2} \Delta \tilde{\mathbf{x}}\right)}+\frac{1}{\rho_{0}\left(\tilde{\mathbf{r}}+\frac{1}{2} \Delta \tilde{\mathbf{y}}\right)}+\frac{1}{\rho_{0}\left(\tilde{\mathbf{r}}-\frac{1}{2} \Delta \tilde{\mathbf{y}}\right)}} \\
& -p(\tilde{\mathbf{r}}, n-1) .
\end{aligned}
$$

Using the definition of specific acoustic impedance, $Z=\rho_{0} c_{n}$, Eq. 4.50 can be expressed in terms of impedance by multiplying the numerator and denominator for the speed of sound $c_{n}$ : 


$$
\begin{aligned}
p(\tilde{\mathbf{r}}, n+1) & =\frac{2\left(\frac{p(\tilde{\mathbf{r}}+\Delta \tilde{\mathbf{x}}, n)}{Z\left(\tilde{\mathbf{r}}+\frac{1}{2} \Delta \tilde{\mathbf{x}}\right)}+\frac{p(\tilde{\mathbf{r}}-\Delta \tilde{\mathbf{x}}, n)}{Z\left(\tilde{\mathbf{r}}-\frac{1}{2} \Delta \tilde{\mathbf{x}}\right)}+\frac{p(\tilde{\mathbf{r}}+\Delta \tilde{\mathbf{y}}, n)}{Z\left(\tilde{\mathbf{r}}+\frac{1}{2} \Delta \tilde{\mathbf{y}}\right)}+\frac{p(\tilde{\mathbf{r}}-\Delta \tilde{\mathbf{y}}, n)}{Z\left(\tilde{\mathbf{r}}-\frac{1}{2} \Delta \tilde{\mathbf{y}}\right)}\right)}{\frac{1}{Z\left(\tilde{\mathbf{r}}+\frac{1}{2} \Delta \tilde{\mathbf{x}}\right)}+\frac{1}{Z\left(\tilde{\mathbf{r}}-\frac{1}{2} \Delta \tilde{\mathbf{x}}\right)}+\frac{1}{Z\left(\tilde{\mathbf{r}}+\frac{1}{2} \Delta \tilde{\mathbf{y}}\right)}+\frac{1}{Z\left(\tilde{\mathbf{r}}-\frac{1}{2} \Delta \tilde{\mathbf{y}}\right)}} \\
& -p(\tilde{\mathbf{r}}, n-1) .
\end{aligned}
$$

It should be noted how for a constant impedance $Z(\tilde{\mathbf{r}})=Z$, Eq. 4.51 becomes Eq. 4.37, after changing a factor $1 / 2$ for $1 / 3$, in account of the change from $2-\mathrm{D}$ to $3-\mathrm{D}$.

\subsection{The Digital Waveguide Mesh (DWM) method}

The theory of the DWM is based on decomposing the solution to the wave equation into travelling (or diverging and converging) waves, in a similar fashion to the d'Alembert solution of the wave equation [Pierce, 1994]. In an ideal lossless medium, the wave equation can be implemented by means of pure delays, and the total sound field is obtained by adding these travelling wave components.

The basis of the DWM arises from the Digital Wave Guide (DWG) method proposed to solve the 1-D wave equation [Smith, 1992]. In the simple 1-D case, the d'Alembert solution is

$$
\psi(x, t)=\psi^{-}(x-c t)+\psi^{+}(x+c t) .
$$

This can be seen as a decomposition into a direct wave $\psi^{+}$, and a reflected wave $\psi^{-}$, in a medium with the speed of sound $c$. Each travelling wave can be simulated by a shift register using pure or fractional delays [Laakso et al., 1996]. In this way, the digital waveguide model is obtained by sampling both space and time. Spatial sampling points are known as scattering junctions in the technical literature [Murphy et al., 2007].

In the multidimensional case, the possible wave directions are an infinite number. In order to simplify the method, the first approach has been to consider only the characteristic directions, derived from the cartesian DWM [Duyne and Smith, 1993]. In Fig. 4.4(a), the scattering junction $p\left(\tilde{\mathbf{r}}_{J}, n\right)$ of a generic DWM, at a discrete time step $n$, is illustrated. This junction is linked by means of bi-directional unit-delays to $N$ neighbouring 


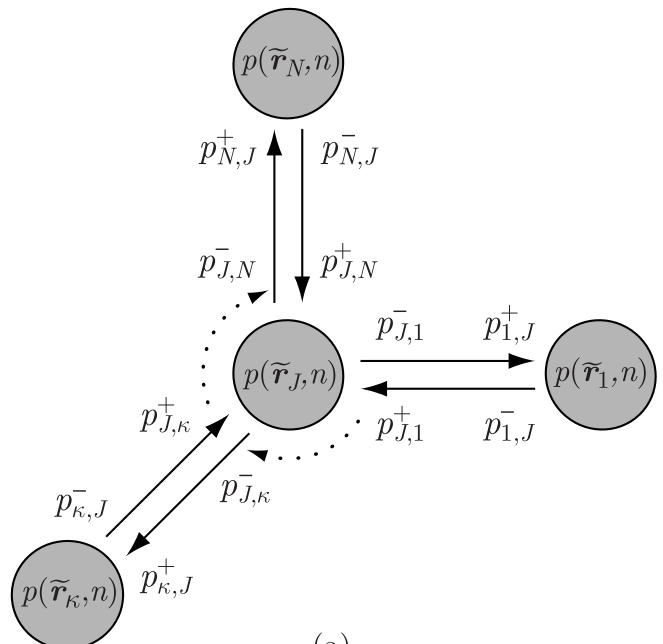

(a)

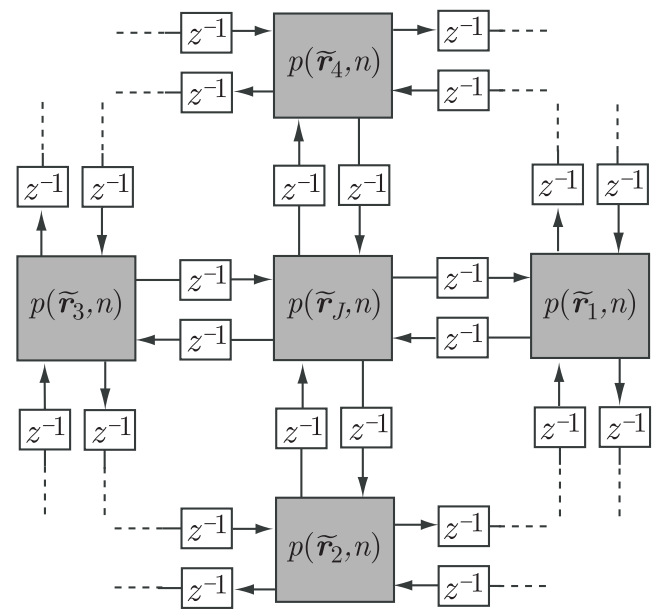

(b)

Figure 4.4. (a) Scattering junctions scheme in a digital waveguide mesh. (b) Implementation of a 2-D Cartesian DWM with digital signal processing theory.

scattering junctions defined with subindex $\kappa, p\left(\tilde{\mathbf{r}}_{\kappa}, n\right)$, where $\kappa=1, \ldots, N$ (see Fig. 4.4(a)). The discrete pressure value $p\left(\tilde{\mathbf{r}}_{J}, n\right)$, is located at the cen- 
tre of the figure, and is surrounded by $N$ neighbouring scattering junctions, which are represented by $p\left(\tilde{\mathbf{r}}_{\kappa}, n\right)(\kappa=1, \ldots, N)$. As shown in Fig. 4.4(b), a generic scheme can be implemented as a cartesian 2-D DWM based on digital signal processing theory where scattering junctions are joined using digital delays, represented by $z^{-1}$.

For a given scattering junction at position $\tilde{\mathbf{r}}_{J}$, let the signal $p_{J, \kappa}^{+}(n)$ be an incoming signal from the neighbouring junction situated at $\tilde{\mathbf{r}}_{\kappa}$ and $p_{J, \kappa}^{-}(n)$ be an outgoing component. Note that delay lines $p_{J, \kappa}^{+}(n)$ and $p_{J, \kappa}^{-}(n)$ join $p\left(\tilde{\mathbf{r}}_{J}, n\right)$ and $p\left(\tilde{\mathbf{r}}_{\kappa}, n\right)$ (see Fig. 4.4(a)). As the delay elements are bidirectional, the pressure is defined as

$$
p\left(\tilde{\mathbf{r}}_{J}, n\right)=p_{J, \kappa}^{+}(n)+p_{J, \kappa}^{-}(n), \forall \kappa \in[1, N] .
$$

In a similar fashion, the particle velocity have an incoming, $u_{J, \kappa}^{+}(n)$, and outgoing signal, $u_{J, \kappa}^{-}(n)$, decomposition ${ }^{2}$. Note that these particle velocity have a non specified direction; it will depend on the direction given by the link between the positions $\tilde{\mathbf{r}}_{J}$ and $\tilde{\mathbf{r}}_{\kappa}$, these directions are not necessarily related to the cartesian ones [Duyne and Smith, 1993, 1995].

In a lossless medium, the Kirchhoff laws, or conservation of mass and momentum (and thus, energy), must hold

1. The sum of incoming particle velocities is equal to the sum of outgoing particle velocities at each junction (conservation of the mass flow).

2. The pressures in all crossing waveguides are equal at the junction (equilibrium of pressure).

Furthermore, let us consider only plane waves. According that assumption, let us define the scattering junction port impedance, $Z_{J, \kappa}$, as the specific impedance that exists in the link between the scattering junction in positions $\tilde{\mathbf{r}}_{J}$ and $\tilde{\mathbf{r}}_{\kappa}$, defined as

\footnotetext{
${ }^{2}$ Original texts about DWM use scalar volume velocity instead of particle velocity vector. The volume velocity represents the time rate of change of the volume enclosed by the surface of its outward-normal velocity and that it is related to, i.e., loudspeakers. This variable could be used for 1-D simulation of strings or tubes (one propagation direction is assumed); but in 2-D or 3-D cases, there does not exist any surface of propagation. Then, it is more rigorous if the particle velocity is used instead, although the final theory does not change indeed.
} 


$$
Z_{J, \kappa}=\frac{p_{J, \kappa}^{+}(n)}{u_{J, \kappa}^{+}(n)}=\frac{p_{J, \kappa}^{-}(n)}{u_{J, \kappa}^{-}(n)} .
$$

Furthermore, note $Z_{J, \kappa}=Z_{\kappa, J}$.

Then, for a given direction $\overrightarrow{J, \kappa}$ equal to the one formed by a given link between the scattering junction at positions $\tilde{\mathbf{r}}_{J}$ and $\tilde{\mathbf{r}}_{\kappa}$, the particle velocity component is calculated as

$$
u_{\overrightarrow{J, \kappa}}\left(\tilde{\mathbf{r}}_{J}, n\right)=\frac{p_{J, \kappa}^{-}(n)-p_{J, \kappa}^{+}(n)}{Z_{J, \kappa}}=u_{J, \kappa}^{-}(n)-u_{J, \kappa}^{+}(n) .
$$

From the first Kirchhoff law it follows that

$$
\sum_{\kappa=1}^{N} \frac{p_{J, \kappa}^{+}(n)}{Z_{J, \kappa}}=\sum_{\kappa=1}^{N} \frac{p_{J, \kappa}^{-}(n)}{Z_{J, \kappa}},
$$

and using Eqs. 4.53 and 4.56, the following expression is achieved

$$
\sum_{\kappa=1}^{N} \frac{p_{J, \kappa}^{+}(n)}{Z_{J, \kappa}}=\sum_{\kappa=1}^{N} \frac{p\left(\tilde{\mathbf{r}}_{J}, n\right)-p_{J, \kappa}^{+}(n)}{Z_{J, \kappa}} .
$$

According to these rules, the sound pressure at a given scattering junction is obtained as

$$
p\left(\tilde{\mathbf{r}}_{J}, n\right)=\frac{2 \sum_{\kappa=1}^{N} \frac{p_{J, \kappa}^{+}(n)}{Z_{J, \kappa}}}{\sum_{\kappa=1}^{N} \frac{1}{Z_{J, \kappa}}} .
$$

From the second Kirchhoff law the reflected component can be obtained as

$$
p_{J, \kappa}^{-}(n)=p\left(\tilde{\mathbf{r}}_{J}, n\right)-p_{J, \kappa}^{+}(n) .
$$

In the next time step, the outgoing components are incoming in the opposite direction. This can be expressed as 


$$
p_{J, \kappa}^{+}(n)=p_{\kappa, J}^{-}(n-1),
$$

where $p_{\kappa, J}^{-}(n)$ represents the outgoing delay line with respect to the scattering junction $p\left(\tilde{\mathbf{r}}_{\kappa}, n\right)$ that links with $p\left(\tilde{\mathbf{r}}_{J}, n\right)$.

It has to be noted how this method has been generalized in a $N$-port mesh. This generalization allows the creation of complex mesh structures, giving different and better grid dispersion and improved efficiencies. The main framework of this work can be found in [Bilbao, 2001, 2004], highly oriented to plate and membranes modeling. To review these alternative meshes is far from the scope of this thesis. From now on, the scheme followed throughout this thesis will be the cartesian one, which means $N=4$ in a 2-D mesh and $N=6$ in a 3-D mesh, what is obtained is a DWM in a cartesian axis. Regarding stability and dispersion, this scheme follows exactly the same properties as the FDTD method. In fact, as will be shown in Sec. 4.4, both methods are equivalent in results, but with different computational characteristics.

\subsubsection{Plane waves in the DWM model}

One of the main considerations in most of the assumptions of this thesis is that only plane waves must be considered. This can be assumed if the source is considerably separated from the impedance surface [Butov, 1981]. On the other hand, the DWM methods also assumes that plane waves are travelling in the medium. For that reason, some considerations should be made about this point.

Let us assume an incident plane wave travelling in $x<0$ towards $x=$ 0 . This plane wave has, for a given discrete position and time step, an amplitude $p_{i}(\tilde{\mathbf{r}}, n)$ and a particle velocity vector associated $\mathbf{u}_{i}(\tilde{\mathbf{r}}, n)$ (see Fig. 4.5 for details). Now, consider the pressure in a scattering junction $p\left(\tilde{\mathbf{r}}_{J}, n\right)$ located close to an interface with some boundary condition and where the plane wave has struck [Kuttruff, 4th edition, 2000]. According to this and the second Kirchhoff law (Sec. 4.3) and Sec. 2.4.1.3

$$
p\left(\tilde{\mathbf{r}}_{J}, n\right)=p_{i}\left(\tilde{\mathbf{r}}_{J}, n\right)+p_{r}\left(\tilde{\mathbf{r}}_{J}, n\right)=p_{J, \kappa}^{+}(n)+p_{J, \kappa}^{-}(n) \forall \kappa \in[1, N] .
$$

One has to consider that Eq. 4.61 does not necessarily imply that $p_{i}\left(\tilde{\mathbf{r}}_{J}, n\right)=$ $p_{J, \kappa}^{-}(n)$ or $p_{r}\left(\tilde{\mathbf{r}}_{J}, n\right)=p_{J, \kappa}^{+}(n)$. One should realize that observing Fig. 4.3, 


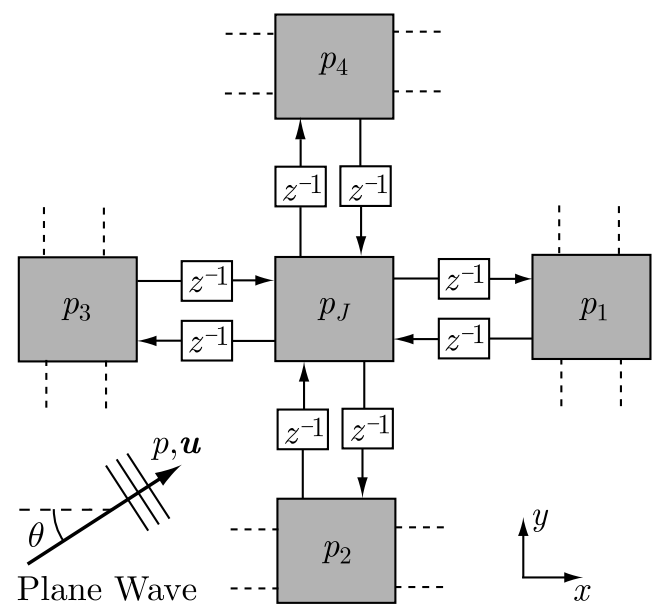

Figure 4.5. A travelling plane wave in a DWM simulation. The particle velocity components are calculated as a vector projection of the components over the links between scattering junctions.

where in case $p_{i}\left(\tilde{\mathbf{r}}_{J}, n\right)=p_{J, \kappa}^{-}(n)$ could be considered, this would mean all outgoing $\kappa$ components would be propagated with the same value and all incoming components would be constant and they would not depend on the neighboring junctions. Another example is when an incident plane wave travels; in that case, the term $p_{r}\left(\tilde{\mathbf{r}}_{J}, n\right)$ will be removed from Eq. 4.61; in that case, there exists incoming component (see Sec. 4.3), and thereby incoming and outgoing DWM components cannot be strictly identified with incident and reflected wave components. Of course, it should be emphasized how this equivalence would become true in the multidimensional case: in a 1-D case, there must be defined $p_{i}\left(\tilde{\mathbf{r}}_{J}, n\right)=p_{J, \kappa}^{-}(n)$ and $p_{r}\left(\tilde{\mathbf{r}}_{J}, n\right)=p_{J, \kappa}^{+}(n)$.

Regarding the particle velocity components in the DWM, $u_{\overrightarrow{J, \kappa}}(\tilde{\mathbf{r}}, n)$, they are calculated through Eq. 4.55. In that case, the particle velocity in the DWM can be related directly to the plane wave particle velocity just using a simple vector projection over the DWM axis. For instance, the total particle velocity over $x$-component (see Fig. 4.5) is determined by $u_{x}\left(\tilde{\mathbf{r}}_{J}, n\right)=u_{\overrightarrow{J, 1}}\left(\tilde{\mathbf{r}}_{J}, n\right)$. Then, this plane wave particle velocity component can be related to the DWM particle velocity component as 


$$
u_{x}\left(\tilde{\mathbf{r}}_{J}, n\right)=\frac{p_{i}\left(\tilde{\mathbf{r}}_{J}, n\right)-p_{r}\left(\tilde{\mathbf{r}}_{J}, n\right)}{\rho_{0} c} \cos \theta=\frac{p_{J, 1}^{-}\left(\tilde{\mathbf{r}}_{J}, n\right)-p_{J, 1}^{+}\left(\tilde{\mathbf{r}}_{J}, n\right)}{Z_{1}} .
$$

It must be taken into account that the equality in Eq. 4.62 could not be completely accurate, due to the dispersion error, which depends on the plane wave frequency and angle [Fontana and Rocchesso, 2001]. However, if high enough oversampling is employed (Eq. 4.36), the error can be assumed negligible. With this technique, Eq. 4.62 can be considered valid up to a fixed frequency. In practice, $2 \times$ oversampling is commonly used [Duyne and Smith, 1993].

\subsection{Equivalence between the FDTD and DWM meth- ods}

As has been mentioned throughout this thesis, both FDTD and DWM, in cartesian coordinates, are equivalent [Duyne and Smith, 1993]. In this section, this equivalence is demonstrated through a 2-D mesh in an inhomogeneous medium defined according Eq. 4.58 and expressing in terms of finite differences (Eq. 4.51). Usually, this equivalence is demonstrated for a homogeneous medium [Murphy, 2000, Duyne, 2007]. However, through some simple modifications, this equivalence can be generalized.

Let us consider the mesh notation of the Fig. 4.4b). Then, the scattering junction pressure at the position $\tilde{\mathbf{r}}_{J}$ can be calculated through Eq. 4.58. By using Eq. 4.59 and Eq. 4.60, it may be found

$$
\begin{aligned}
p_{J, \kappa}^{+}(n) & =p_{\kappa, J}^{-}(n-1) \\
& =p\left(\tilde{\mathbf{r}}_{\kappa}, n-1\right)-p_{\kappa, J}^{+}(n-1) \\
& =p\left(\tilde{\mathbf{r}}_{\kappa}, n-1\right)-p_{J, \kappa}^{-}(n-2) \\
& =p\left(\tilde{\mathbf{r}}_{\kappa}, n-1\right)-p\left(\tilde{\mathbf{r}}_{J}, n-2\right)+p_{J, \kappa}^{+}(n-2) .
\end{aligned}
$$

Returning to the definition of the pressure in a scattering junction as a sum of the incoming pressure components, Eq. 4.58, let us substitute the Eq. 4.63 on it. 


$$
\begin{aligned}
& p\left(\tilde{\mathbf{r}}_{J}, n\right)=\frac{2 \sum_{\kappa=1}^{N} \frac{p_{J, \kappa}^{+}(n)}{Z_{J, \kappa}}}{\sum_{\kappa=1}^{N} \frac{1}{Z_{J, \kappa}}} \\
& =\frac{2 \sum_{\kappa=1}^{N} \frac{p\left(\tilde{\mathbf{r}}_{\kappa}, n-1\right)-p\left(\tilde{\mathbf{r}}_{J}, n-2\right)+p_{J, \kappa}^{+}(n-2)}{Z_{J, \kappa}}}{\sum_{\kappa=1}^{N} \frac{1}{Z_{J, \kappa}}} \\
& =2 \frac{\sum_{\kappa=1}^{N} \frac{p\left(\tilde{\mathbf{r}}_{\kappa}, n-1\right)}{Z_{J, \kappa}}-\sum_{\kappa=1}^{N} \frac{p\left(\tilde{\mathbf{r}}_{J}, n-2\right)}{Z_{J, \kappa}}+\sum_{\kappa=1}^{N} \frac{p_{J, \kappa}^{+}(n-2)}{Z_{J, \kappa}}}{\sum_{\kappa=1}^{N} \frac{1}{Z_{J, \kappa}}} .
\end{aligned}
$$

Noting that

$$
\begin{aligned}
& \frac{\sum_{\kappa=1}^{N} \frac{p\left(\tilde{\mathbf{r}}_{J, n-2}\right)}{Z_{J, \kappa}}}{\sum_{\kappa=1}^{N} \frac{1}{Z_{J, \kappa}}}=p\left(\tilde{\mathbf{r}}_{J}, n-2\right), \\
& \frac{\sum_{\kappa=1}^{N} \frac{p_{J, \kappa}^{+}(n-2)}{Z_{J, \kappa}}}{\sum_{\kappa=1}^{N} \frac{1}{Z_{J, \kappa}}}=\frac{p\left(\tilde{\mathbf{r}}_{J}, n-2\right)}{2},
\end{aligned}
$$

a final expression obtained is

$$
p\left(\tilde{\mathbf{r}}_{J}, n\right)=\frac{2 \sum_{\kappa=1}^{N} \frac{p\left(\tilde{\mathbf{r}}_{\kappa}, n-1\right)}{Z_{J, \kappa}}}{\sum_{\kappa=1}^{N} \frac{1}{Z_{J, \kappa}}}-p\left(\tilde{\mathbf{r}}_{J}, n-2\right) .
$$

With a simple inspection of Fig. 4.4, the next terms between Eq. 4.67 and Eq. 4.51 can be identified:

$$
\begin{aligned}
& p\left(\tilde{\mathbf{r}}_{1}, n\right)=p(\tilde{\mathbf{r}}+\Delta \tilde{\mathbf{x}}, n), \\
& p\left(\tilde{\mathbf{r}}_{2}, n\right)=p(\tilde{\mathbf{r}}-\Delta \tilde{\mathbf{y}}, n), \\
& p\left(\tilde{\mathbf{r}}_{3}, n\right)=p(\tilde{\mathbf{r}}-\Delta \tilde{\mathbf{x}}, n), \\
& p\left(\tilde{\mathbf{r}}_{4}, n\right)=p(\tilde{\mathbf{r}}+\Delta \tilde{\mathbf{y}}, n) .
\end{aligned}
$$


At this point, an interesting question arises: are the FDTD and the DWM equivalent in an inhomogeneous media? The unstaggered nature of the DWM situates the impedance (also the particle velocity components) at the same position as the pressure, however the FDTD does not (see Sec. 4.2). However, what might be considered is that each delay line has a constant impedance, at both sides of the delay. Then, one can force the equivalence

$$
\begin{aligned}
& Z_{J, 1}=Z\left(\tilde{\mathbf{r}}+\frac{1}{2} \Delta \tilde{\mathbf{x}}\right), \\
& Z_{J, 2}=Z\left(\tilde{\mathbf{r}}-\frac{1}{2} \Delta \tilde{\mathbf{y}}\right), \\
& Z_{J, 3}=Z\left(\tilde{\mathbf{r}}-\frac{1}{2} \Delta \tilde{\mathbf{x}}\right), \\
& Z_{J, 4}=Z\left(\tilde{\mathbf{r}}+\frac{1}{2} \Delta \tilde{\mathbf{y}}\right) .
\end{aligned}
$$

It should be remembered that the impedance in a DWM is a delay line property, not from the scattering junction itself, since one can be linked to a different delay line with different media properties. This can also provide problems in the interface position of two different media and an important lack of accuracy can be made in the simulation. Let us consider a simple case where $Z(\tilde{\mathbf{r}}-\Delta \tilde{\mathbf{x}}) \neq Z(\tilde{\mathbf{r}})$, the doubt arises when one has to define $Z\left(\tilde{\mathbf{r}}_{-\frac{1}{2}} \Delta \tilde{\mathbf{x}}\right)$. A simple way to face the problem consist of averaging both values and to assume a linear transition of the densities, but it can be fairly inaccurate in some cases [Yefet and Petropoulous, 1999, Beggs, 2001]. However, this is not problematic in the room acoustic case, where the medium could be considered homogeneous, except scenarios where there exist a considerable gradient of temperature.

According to Eqs. 4.72, the equivalence between the FDTD and DWM in an inhomogeneous medium is demonstrated. Then, the same equivalence for the homogenous media case is straightforward.

Since these methods are equivalent and as indicated in Sec. 3.4.1, during the last years, a new nomenclature has arisen to identify both methods: whereas the method based on incoming and outgoing variables (Eq. 4.58) is also known as Wave-DWM or W-DWM, the method based on the the pressure in the scattering junction (Eqs. 4.51 and 4.37) is named as the Kirchhoff-DWM (or K-DWM) [Karjalainen, 2004, Erkut and Karjalainen, 2002b, Välimäki et al., 2006]. In accordance with this definition, the KDWM and the Savioja's FDTD methods (see Sec. 4.2.3) are equivalent, 
whereas the DWM (according Sec. 4.3) and the W-DWM are also equivalent. As long as this new nomenclature has been widely used in room acoustic modeling and physical modeling-based digital sound synthesis, from now on in this thesis and unless otherwise indicated, the FDTD will be only used for the Botteldooren's approach (see Sec. 4.2); and the DWM acronym will be specified if it corresponds with the K- or W- approach, being the K-DWM equivalent to Savioja's approach.

Besides this equivalence between both approaches, several different properties differentiate between them [Karjalainen, 2004]:

\section{K-DWM}

- K-DWM is quite more efficient than W-DWM.

- It can incorporate non-frequency dependent losses, just modifying the wave equations.

- It does not assume initial conditions or the propagating wave shape; then, they appear as a result of the numerical approximation of the wave equation.

\section{W-DWM}

- Digital signal processing theory is suitable to be used in W-DWM (frequency-dependent boundary conditions, frequency-dependent losses, ....).

- W-WDM avoids spurious responses to specific excitations [Smith, 1998].

A deep comparison of both methods can be found in [Bilbao, 2001, Erkut and Karjalainen, 2002a, Karjalainen et al., 2003].

However, an aspect that has not been analyzed of the equivalence between both methods is that regarding particle velocity. The DWM method always assumes a plane wave propagation (Eq. 4.54), but this is hard to assume near a point source. However, the FDTD method does not assume any wave shape (Eqs. 4.14-4.16). Despite the results of the pressure simulation, if one simulates the particle velocity vector of a point source (see Fig. 4.6), some differences can be observed at positions situated near the source, but when the components are situated at a certain distance from the source, no differences are observed. In a relatively large simulation, 
this does not cause any inaccuracy of the methods. Although this point is far from the scope of this thesis, it could be an interesting contribution to understand these methods with more detail.
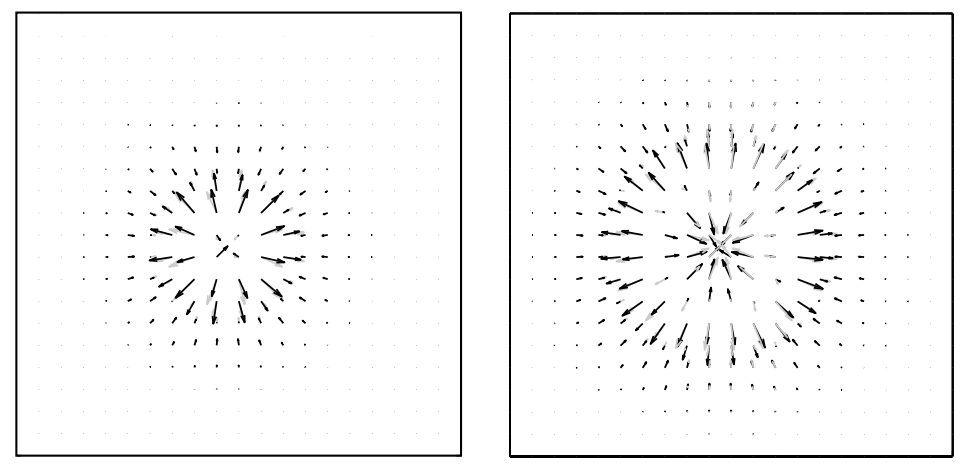

Figure 4.6. Simulation of the particle velocity vector through FDTD method (grey arrow) and DWM method (black arrow).

\subsection{Hybrid models}

Since both approaches are equivalent in results but with different advantages, it is desirable to find a method which combines the advantages of both K- and W-DWM: in terms of efficiency, K-DWM reduces the computational cost considerably, whereas the W-DWM has traditionally been used to adapt boundary conditions to the DWM [Savioja et al., 1995, Huopaniemi et al., 1997]. The use of $K W$-pipes [Smith, 2004, Karjalainen and Erkut, 2004, Murphy and Beeson, 2007] has permitted the creation of hybrid models combining the advantages of both methods. These KW-pipes are a signal transformation allowing the interface of two meshes built with different methods. A wave should travel from one mesh to another with no energy losses in the interface.

The idea behind these models is, given two scattering junctions one of which belongs to the K-DWM, $p\left(\tilde{\mathbf{r}}_{k}, n\right)$, and the other one belongs to the W-DWM, $p\left(\tilde{\mathbf{r}}_{w}, n\right)$, to find a suitable transformation for the energy transition without loss of the wave propagation, taking into account that $p\left(\tilde{\mathbf{r}}_{k}-\Delta \tilde{\mathbf{x}}, n\right)=p\left(\tilde{\mathbf{r}}_{w}, n\right)$ and $p\left(\tilde{\mathbf{r}}_{w}+\Delta \tilde{\mathbf{x}}, n\right)=p\left(\tilde{\mathbf{r}}_{k}, n\right)$, according Fig. 4.7. 


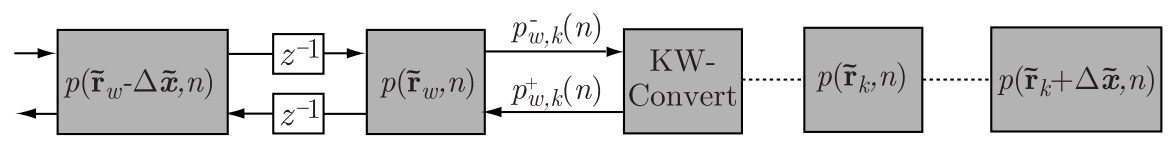

Figure 4.7. Generic scheme of a 1-D hybrid model.

In the particular case of room acoustic simulation, a hybrid model is a very interesting solution. An hybrid model based on the use of KW-pipes allows us to obtain an efficient model using the K-DWM as the main method, but the use of a W-DWM in the interface with the boundary conditions permits the inclusion of frequency-dependent models with a highly reduced complexity. More details about the boundary conditions will be addressed in Chapter 5.

There exist three approaches to the hybrid model: Smith [Smith, 2004] proposes an analysis on the equivalence between both schemes through a formulation based on Space State Systems (SSS); this conversion allows the conversion of the state variables of a K-DWM (FDTD in the original) to the corresponding wave variables in a W-DWM. Recently, Murphy and Besson have proposed a highly versatile approach to this problem, giving a solution to mix both methods in an hybrid triangular DWM [Murphy and Beeson, 2007], which gives a highly efficient hybrid model.

However, the most popular approach to this problem has been the one proposed by Karjalainen and Erkut [Karjalainen and Erkut, 2004]. Originally, their KW-pipe has been designed to simulate a string (1-D), consisting of half string with a Kirchhoff Digital Waveguide (FDTD in the original) and a Wave Digital Waveguide (DWG in the original), according to the matrix transformation of Eq. 4.73.

$$
\left(\begin{array}{c}
p_{w, k}^{+}(n) \\
p\left(\tilde{\mathbf{r}}_{w}, n-1\right)
\end{array}\right)=\left(\begin{array}{cc}
1 & -z^{-2} \\
1 & 1-z^{-2}
\end{array}\right) \cdot\left(\begin{array}{c}
p\left(\tilde{\mathbf{r}}_{k}, n-1\right) \\
p_{w, k}^{-}(n)
\end{array}\right)
$$

From Fig. 4.7 and Eq. 4.37, $p\left(\tilde{\mathbf{r}}_{k}, n\right)$ is directly updated as 


$$
p\left(\tilde{\mathbf{r}}_{k}, n\right)=p\left(\tilde{\mathbf{r}}_{w}, n-1\right)+p\left(\tilde{\mathbf{r}}_{k}+\Delta \tilde{\mathbf{x}}, n-1\right)-p\left(\tilde{\mathbf{r}}_{k}, n-2\right)
$$

However, this KW-pipe also allows the creation of a cartesian hybrid mesh, just interfacing through the pipe, one to one, the contiguous scattering junctions, as shown in Fig. 4.8. It is true for curved boundaries, where the perimeter of the structure being modelled (such as a drum membrane) is not normal/parallel to the axes of the mesh structure, but this KW-pipe could be inappropriate and less accurate than the proposed one in [Murphy and Beeson, 2007, Laird et al., 1999]; however, under the scope of this thesis, only cartesian axis-based meshes are considered and the efficiency and simplicity of this KW-pipe has been noted as the appropriate one.

The key point on the use of hybrid mesh lies in the design of more efficient mesh, since it is possible to define the air propagation in a $\mathrm{K}$ DWM due to its efficiency and the boundaries through W-DWM at the discretized points where there exist walls (see Sec. 5.2.1 for details).

\subsection{Sound propagation simulation through discrete- time methods}

Throughout this chapter, the mathematical formulation of the FDTD and DWM (including both K- and W-DWM) methods from physical principles of the sound propagation has been deduced. It is reasonable to think that most of the wave propagation phenomenons may appear during the simulation, except air sound absorption.

Through this section, a few examples of the most common wave phenomenons are simulated with those methods, demonstrating the suitability of the methods for room acoustic simulation.

The first example concerns the multilayer transmission and reflection. Variations of the density or the speed of sound at different areas of the space under analysis give as a result a set of reflections and transmissions in the interface between the different specific impedances. Let us consider two different media, with specific impedance $Z_{1}$ and $Z_{2}$. The proportion of the absorbed energy is $|R|^{2}$, where $R$ is calculated by 


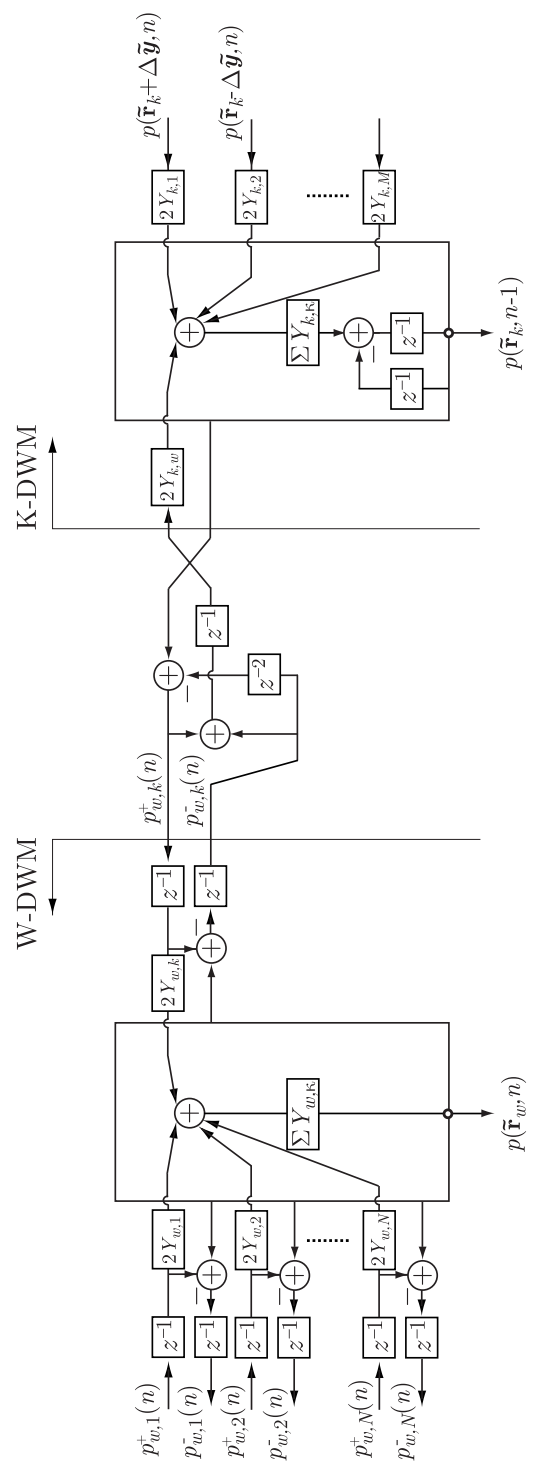

Figure 4.8. Functional block diagram of a hybrid mesh consisting of a generic multidimensional K-DWM and $W-D W M$, interfaced by a $K W$-pipe. The impedances have been expressed in terms of their correspondent admittances. (Reproduced with permission of the authors of [Murphy and Beeson, 2007]) 

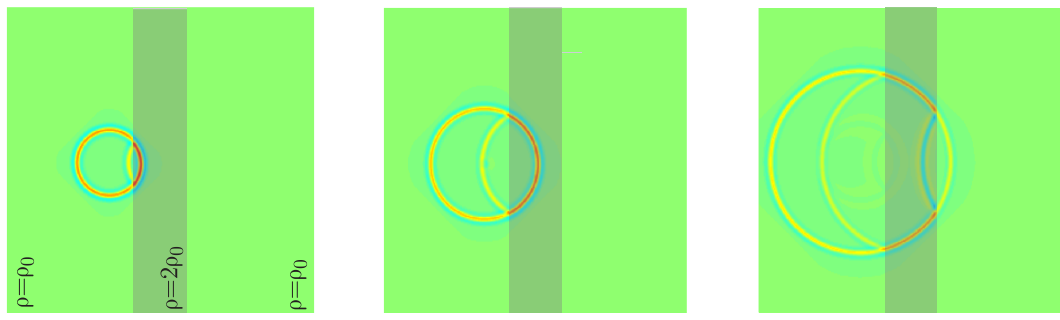

Figure 4.9. Different screenshots of a sound wave propagation through two different layers with different specific impedance given by changes in density.

$$
R=\frac{Z_{2}-Z_{1}}{Z_{2}+Z_{1}}
$$

whereas the transmitted energy through the interface is $1-|R|^{2}$. In room acoustic simulation in cases where a large size room is analyzed, it should be considered that gradients of temperature could appear, changing the specific impedance at different areas.

Let us consider a simulation through the K-DWM method in an inhomogeneous medium, with two different densities, $\rho_{1}=\rho_{0}$ and $\rho_{2}=2 \rho_{0}{ }^{3}$. Figure 4.9 shows a simulation at different time steps, where the reflection and transmission through different specific impedances is observed.

Another interesting phenomenon is diffraction, which is defined as the bending of waves around small obstacles and the spreading out of waves beyond small openings ${ }^{4}$. Let us define a homogeneous 2 -D mesh with a sampling frequency $f_{s}=20 \mathrm{kHz}$ and speed of sound $c=341 \mathrm{~m} / \mathrm{s}$, and according Eq. 4.36, each discretized point has a size of $1.2 \mathrm{~cm}^{2}$. Let us also define a square obstacle of $24 \mathrm{~cm}^{2}(20 \times 20$ cells $)$. Under these conditions, two simulations are carried out with two different monochromatic point sources with frequency $f_{1}=200 \mathrm{~Hz}\left(\lambda_{1}=1.7 \mathrm{~m}\right)$ and $f_{2}=2500 \mathrm{~Hz}\left(\lambda_{1}=0.13 \mathrm{~m}\right)$. Note that $\lambda_{1}$ is bigger than the size of the obstacle; then, what is expected is that the wave passes through the obstacle and apparently, it is almost

\footnotetext{
${ }^{3}$ Since the equivalence between both FDTD and DWM methods has been demonstrated, the validity of the simulation demonstrating those sound propagation phenomenons in this section is also for the W-DWM and FDTD method.

${ }^{4}$ Small compared to the wavelength
} 
"invisible" to the wave (see Fig. 4.10(a)). However, $f_{2}$ has a wavelength smaller than the obstacle; then, a shadow effect occurs at an opposite point to the source position in regard to the obstacle (see Fig. 4.10(b)).

A common example of the sound diffraction effect is the one which appears in noise barriers, which are frequently used to mitigate the impact of traffic on roadside communities. The screening performance of barriers is dominated by the sound that is diffracted by the top of the barrier. A 2-D simulation under the same conditions as the previous example but with a broadband pulse coming from a point source is carried out, including a barrier. Figure 4.11 shows a screenshot of the simulation where the diffractions are evident around the top of the barrier.

Another known effect of diffraction is the one due to the propagation of a wave through a small opening, which results as a consequence of each particle of a sound wave emitting outward in a spherical fashion ${ }^{5}$ (see Fig. 4.12).

Finally, the (constructive or destructive) superposition of waves is an inherent property of the simulation through these methods, giving as a result a tool for the analysis of room modes [Kuttruff, 4th edition, 2000]. With the same conditions as the previous simulations, a broadband point source radiates and since hard walls are included at boundaries (see Sec.5.2.1), a set of reflections occur producing interference between the waves at different positions; the simulation is shown in Fig. 4.13 at different time steps.

However, it should be mentioned that the dispersion of these methods (see Sec. 4.2.1) produce a misalignment between the room modes resulting from the simulation and those expected, especially at high frequencies [Duyne and Smith, 1993, 1995].

The importance of showing these effects lies, not only in the possibility of observing and analyzing the wave propagation effects and their evolution in time, which is an evident advantage over frequency-based wave methods, but also in the accuracy of the discrete-time methods with a relatively reduced computational cost. Special attention should also be paid to the fact that the geometrical methods do not include these effects in a direct

\footnotetext{
${ }^{5}$ This effect is known as a Huygens's principle, stating the wavefront of a propagating wave of sound at any instant conforms to the envelope of spherical wavelets emanating from every point on the wavefront at the prior instant (with the understanding that the wavelets have the same speed as the overall wave).
} 

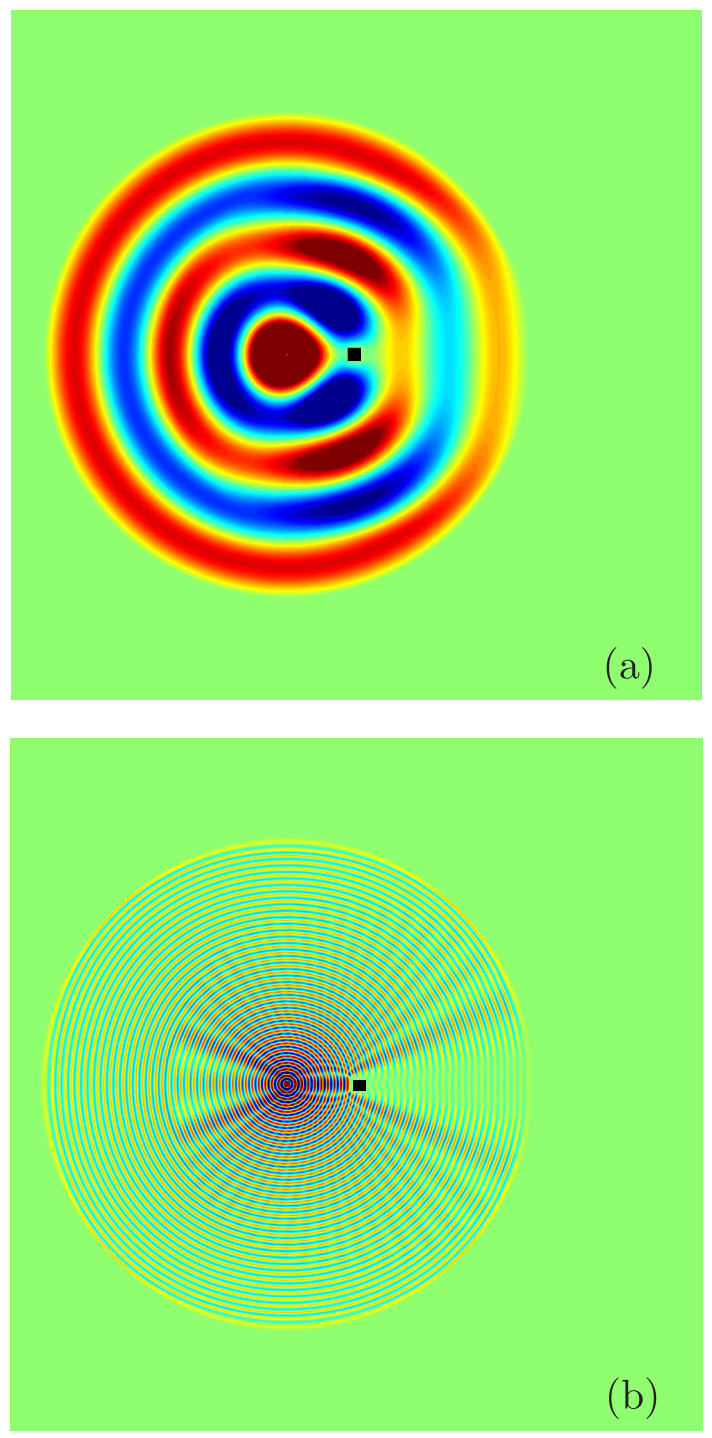

Figure 4.10. Simulation of the diffraction effects due to an obstacle at two different frequencies, (a) $f_{1}=200 \mathrm{~Hz}$ and (b) $f_{2}=2500 \mathrm{~Hz}$.

way, and to include them require a considerable computational effort.

Figure 4.14 shows an example of the suitability of these methods for 


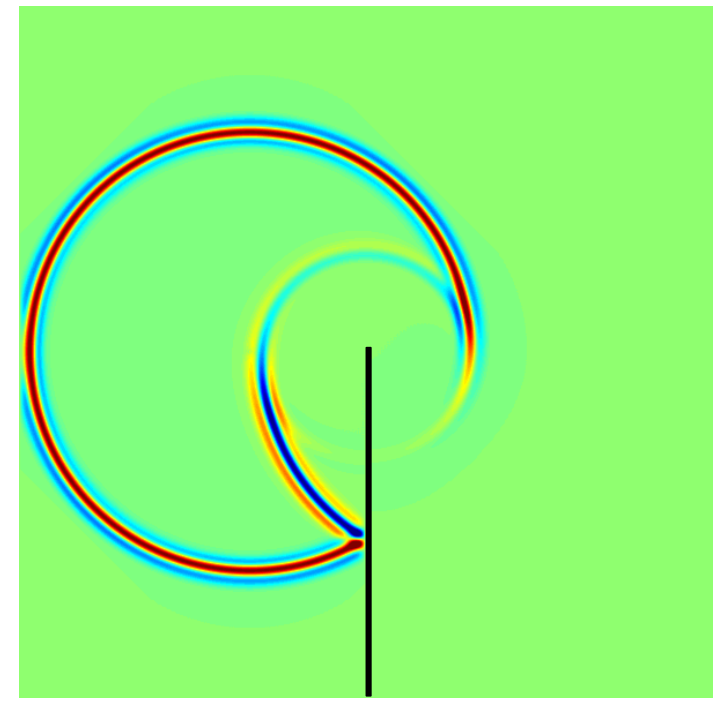

Figure 4.11. Simulation of the effect of edge diffraction in a noise barrier.

room acoustic simulation. In this figure, the enclosure shape has been limited by absorbing walls with $\mathrm{A}=0.75$ (details will be given in Chap. 5 ).

Some details about the computational cost should be mentioned in this section. According the DWM/FDTD theory presented in this chapter, the limiting frequency for a given element size and 3-D simulations with a certain accuracy, is calculated as

$$
f_{\max }=\frac{2 c}{\sqrt{3} \Delta x} \approx \frac{394}{\Delta x} .
$$

It could be interesting to compare it with a FEM simulation: this frequency limit is calculated through (see Sec. 3.3.1)

$$
f_{\max }=\frac{c}{6 \Delta x} \approx \frac{57}{\Delta x}
$$

showing the conclusion the FDTD/DWM algorithm is more efficient than the FEM algorithm regarding the memory storage ${ }^{6}$. Furthermore, it should

\footnotetext{
${ }^{6}$ This conclusion should be assumed with some restrictions, since both limits are
} 


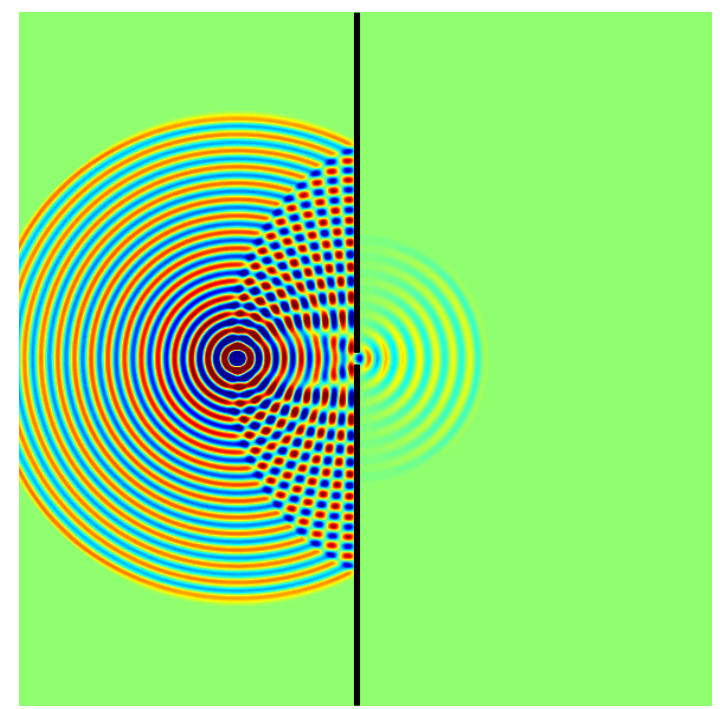

Figure 4.12. Simulation of the diffraction effects of a sound wave propagation through an small opening.

be remembered FEM algorithm needs a simulation for each frequency, whereas FDTD/DWM just need one in order to obtain a simulation in a certain frequency band.

Regarding the computational time, Campos gives a formula for the estimation of that time of a 3-D mesh, based on acoustic characteristics of the room under analysis [Campos, 1999] ${ }^{7}$

$$
T_{M} \approx 0.42 \times 10^{6} \times V \times R T_{60} \times f_{s n}^{4} \times t_{n}
$$

where

calculated assuming different accuracy criteria, given in [van der Geest and McChulloch, 1998] and [Duyne and Smith, 1993]. A comparison between both methods with an unique criteria should be proposed.

${ }^{7}$ Although as far as the author of this thesis knows, this document remains unpublished; however, the formula is reported by Murphy [Murphy, 2000]. 


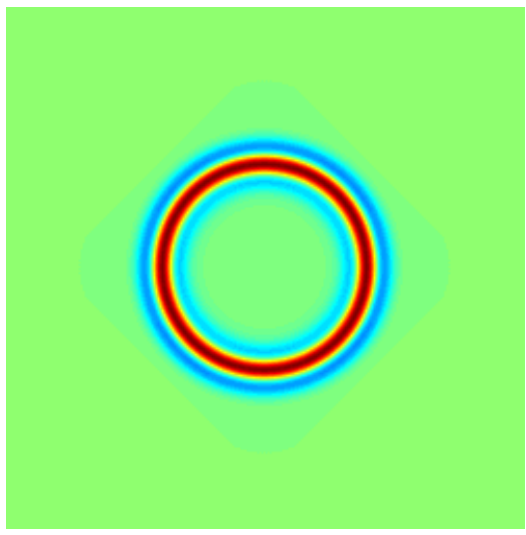

(a)

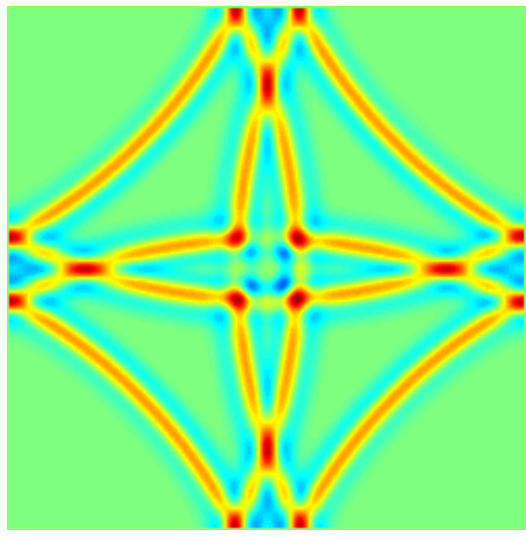

(c)

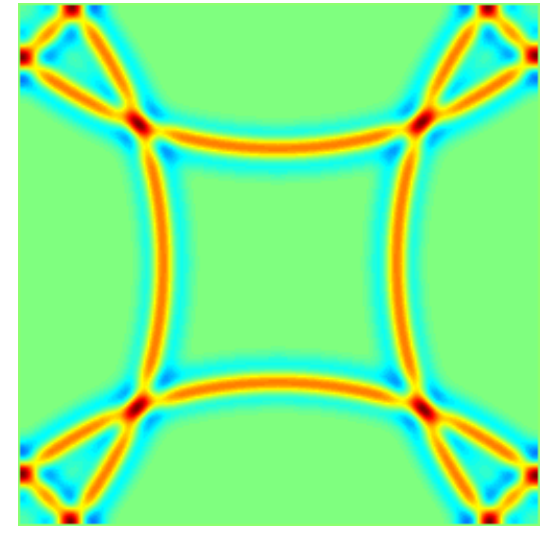

(b)

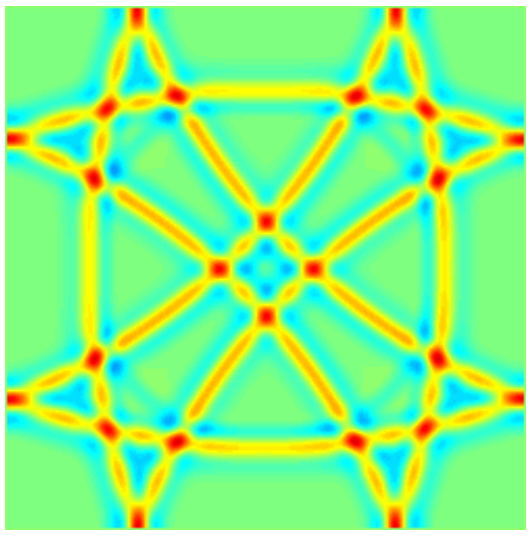

(d)

Figure 4.13. Simulation of the sound propagation where boundaries include hard walls. In the different time steps, it is observed the interferences between the wavefronts, in a constructive and destructive way.

$T_{M} \quad$ Total model computation time in seconds,

$V \quad$ Volume of acoustic space to be modeled in cubic meter,

$R T_{60}$ Reverberation time [Kuttruff, 4th edition, 2000] measured in seconds,

$f_{s n} \quad$ Normalized sampling rate $\left(f_{\max } / 44100\right)$,

$t_{n} \quad$ Average computation time per junction per 44100 iterations, in seconds. 


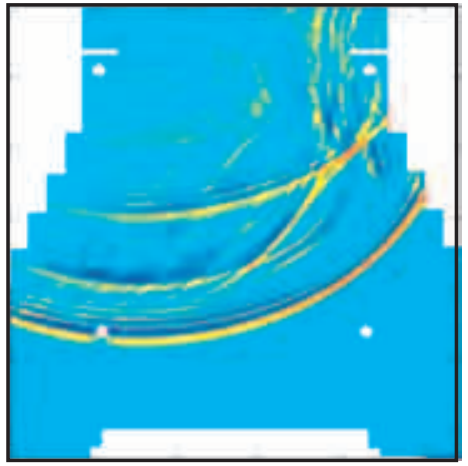

a)

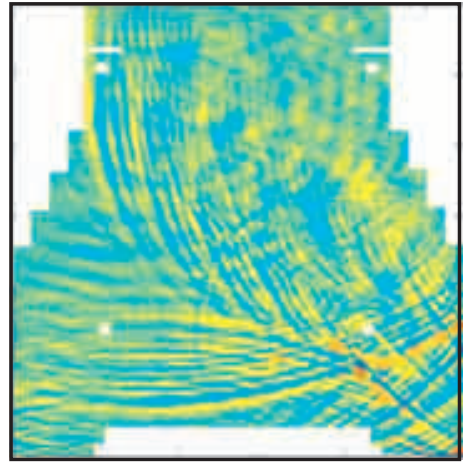

b)

Figure 4.14. Wave propagation simulation of a 2-D enclosure by using the K-DWM method at different time steps.

\subsection{Wave Digital Filters}

In this section, the Wave Digital Filter (WDF) theory [Fettweis, 1986] is introduced. Although it has been used as a room acoustic sound field simulation method (see Sec. 3.4.2), this is not the concern of this thesis. However, as will be seen in Chap. 5, WDFs will be proposed in this thesis to define frequency-dependent boundary conditions. In this section, paying special attention to the digital filter transformation into a WDF system.

\subsubsection{A WDF variables approach}

WDFs appear as a discretization process of complex systems, where each network element is discretized separately by a bilinear transformation [Proakis and Manolakis, 1998]. They provide an approach to digital filter structures that are analogous to analog filter structures that have a certain desirable properties. The main reason for using WDFs is the capacity to avoid potential computational problems, e.g., delay-free loops or potentially unstable implicit equations in the interconnection of the network elements [Szczupak and Mitra, 1975]. The delay-free loop problem is a computational handicap in the implementation of discrete systems. Basically, it occurs when the computation of some values requires knowledge of the same value. A digital structure with delay-free loops is physically impossible to achieve due to the 
finite time required to carry out all arithmetic operations on a computer.

To avoid these delay-free loop problems can be done by using the socalled wave variables,

$$
\begin{aligned}
& a(n)=v(n)+Z_{p} i(n), \\
& b(n)=v(n)-Z_{p} i(n),
\end{aligned}
$$

where the variables $a(n)$ and $b(n)$ represent the outgoing and incoming wave variables, respectively, and propagation information is inherent in them; whereas $v(n)$ and $i(n)$ represent, in the original [Fettweis, 1986], the voltage and intensity. The Kirchhoff variables, $v$ and $i$ can be expressed in terms of the wave variables as

$$
\begin{aligned}
& v(n)=\frac{a(n)+b(n)}{2}, \\
& i(n)=\frac{a(n)-b(n)}{2 Z_{p}} .
\end{aligned}
$$

The proper choice of the port resistance $Z_{p}$ makes it possible to separate the design of the elements from the definition of their interaction by appropriate adaptor elements [Petrausch et al., 2005a]. Here, the nomenclature $Z_{p}$ is used instead the classic $R$ for the resistance in order not to create confusion with the reflection factor. The source of instability, a delay-free loop, is avoided using this variable conversion [Fettweis, 1986].

\subsubsection{Wave digital elements}

Not being in the scope of this thesis to deal deeply with WDFs, it is interesting to review some of their characteristics; however, a deep review of this topic can be found in [Bilbao, 2001].

As mentioned in Sec. 4.7, the WDFs arise from the bilinear transform [Fettweis, 1986], given by

$$
s \longrightarrow \frac{2}{\Delta t} \frac{1-z^{-1}}{1+z^{-1}}
$$


where $s$ is the Laplace variable. It is important to mention that the timedomain interpretation of the bilinear transformation is called the trapezoid rule for numerical integration [Bilbao, 2001].

To see how the WDF works, let us consider circuit elements such as a resistor $(R)$, inductor $(L)$ and a capacitor $(C)$, whose electrical expressions are, respectively

$$
\begin{aligned}
v(t) & =R i(t), \\
v(t) & =L \frac{d i(t)}{d t}, \\
v(t) & =\frac{1}{C} \int_{-\infty}^{t} i(\tau) d \tau,
\end{aligned}
$$

or in the Laplace transformed domain

$$
\begin{aligned}
V(s) & =R I(s) \\
V(s) & =s L I(s), \\
V(s) & =\frac{I(s)}{s C} .
\end{aligned}
$$

After using the bilinear transformation (Eq. 4.83) in Eqs. 4.87-4.89 and expressing them in the discrete-time domain, the following expression are obtained

$$
\begin{aligned}
v(n) & =\operatorname{Ri}(n) \\
v(n) & =\frac{2 L}{\Delta t}(i(n)-i(n-1))-v(n-1), \\
v(n) & =\frac{\Delta t}{2 C}(i(n)+i(n-1))+v(n-1) .
\end{aligned}
$$

Now, let us use the WDF variable transformation (Eqs.5.27 and 5.28) 


$$
\begin{aligned}
b(n) & =\frac{R}{Z_{p}}(a(n)+b(n))-a(n) \\
b(n) & =\frac{2 L}{Z_{p} \Delta t}(a(n)-b(n)-a(n-1)+b(n-1))-a(n) \\
& -a(n-1)-b(n-1), \\
b(n) & =\frac{Z_{p} \Delta t}{2 C}(a(n)-b(n)+a(n-1)-b(n-1))-a(n) \\
& +a(n-1)+b(n-1) .
\end{aligned}
$$

Then, if a choice is made for each one of these equations: $Z_{p}=R$ for Eq. 4.93, $Z_{p}=2 L / \Delta t$ for Eq. 4.94 and $Z_{p}=\Delta t / 2 / C$ for Eq. 4.95 , for each case, what is obtained is

$$
\begin{aligned}
& b(n)=0, \\
& b(n)=-a(n-1), \\
& b(n)=a(n-1) .
\end{aligned}
$$

It has to be noted how, with the WDF variables transformation and the proper choice of port resistance $Z_{p}$, the delay-free loop is avoided and no instantaneous feedback appears.

\subsubsection{Wave digital element interconnection}

So far, basic elements have been converted to WDFs; however, it is reasonable to need to build more complex systems. This can be done through interconnecting basic elements in a combination of parallel and serial structures, in the same fashion as analog filtering, through the so-called port adaptor.

Let us consider $L$ wave digital elements, each one with their variables $a_{k}(n)$ and $b_{k}(n)$, and an impedance port $Z_{p_{k}}$. Following the steps given in [Bilbao, 2001], it is possible to find the equations relating the input and the output wave variables at the $k$ th port for both types of connection as 


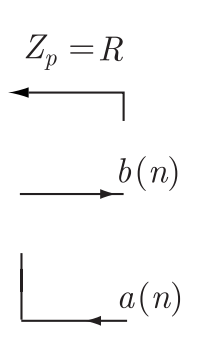

(a)

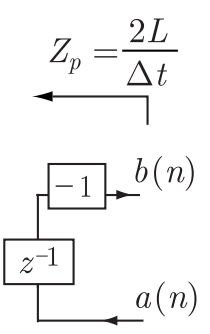

(b)

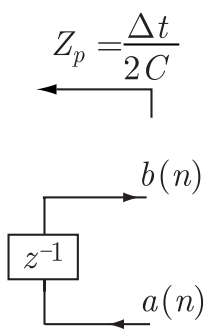

(c)

Figure 4.15. (a) WDF representation of a resistor with resistance $R$, (b) WDF representation of a inductor with inductance $L$ and (c) WDF representation of a capacitor with capacitance $C$.

$$
\begin{aligned}
& b_{k}(n)=a_{k}(n)-\frac{2 Z_{p_{k}}}{\sum_{m=1}^{M} Z_{p_{m}}} \sum_{m=1}^{M} a_{m}(n), \\
& b_{k}(n)=-a_{k}(n)+\frac{2}{\sum_{m=1}^{M} Y_{p_{m}}} \sum_{m=1}^{M} Y_{p_{m}} a_{m}(n),
\end{aligned}
$$

where $k=1 \ldots M$ and $Y_{p_{m}}=1 / Z_{p_{m}}$. Equation 4.99 works for serial connection, whereas Eq. 4.100 is for parallel connection. It is a common practice to represent these adaptors using the blocks of Fig. 4.16

These adaptors allow us to build complex structures as a combination of wave digital elements as serial and/or parallel interconnections. For example, let us consider a resistor $R$ and inductance $L$, defining a serial interaction and a parallel interaction. In accordance with Eq. 4.96 and 4.97, each one of those elements is defined in terms of wave variables, with a wave digital resistance $Z_{p R}=R$ for the resistor and a wave digital resistance $Z_{p L}=2 L / \Delta t$ for the inductor. Then, it is possible to represent a slightly complex WDF structure of a serial and parallel filter represented in Fig. 4.17. More complex filters can be defined through more elaborated combinations of elements.

In this thesis, only 2-port adaptors are used. In accordance with Eq. 4.99 and 4.100 , they can be arranged in matrix form as 


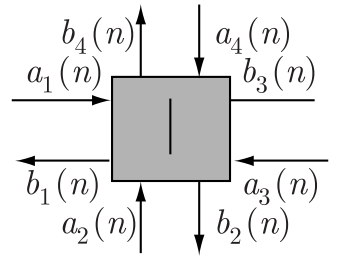

(a)

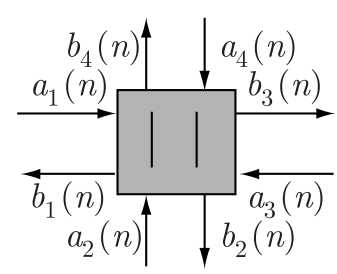

(b)

Figure 4.16. (a) General diagram of a serial WDF 4-port adaptors. (b) General diagram of a parallel WDF 4-port adaptors.
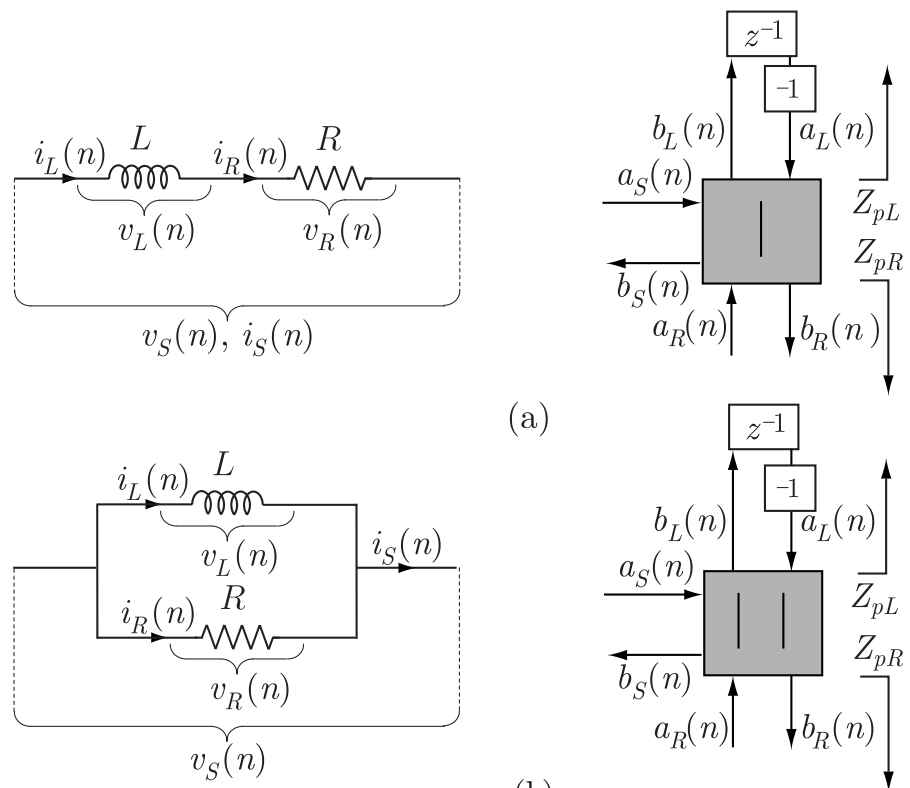

(a)

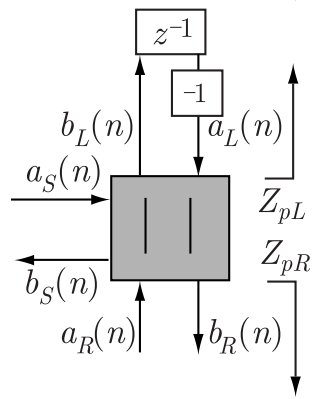

(b)

Figure 4.17. (a) Analog and wave digital structure of a serial filter formed by a resistor $R$ and an inductor $L$. (b) (a) Analog and wave digital structure of a serial filter formed by a resistor $R$ and an inductor $L$. 


$$
\begin{aligned}
& \left(\begin{array}{l}
b_{1}(n) \\
b_{2}(n)
\end{array}\right)=\left(\begin{array}{cc}
\gamma & -(1-\gamma) \\
-(1+\gamma) & -\gamma
\end{array}\right)\left(\begin{array}{l}
a_{1}(n) \\
a_{2}(n)
\end{array}\right), \\
& \left(\begin{array}{l}
b_{1}(n) \\
b_{2}(n)
\end{array}\right)=\left(\begin{array}{cc}
\gamma & 1-\gamma \\
1+\gamma & -\gamma
\end{array}\right)\left(\begin{array}{l}
a_{1}(n) \\
a_{2}(n)
\end{array}\right),
\end{aligned}
$$

where $\gamma=\left(Z_{p 2}-Z_{p 1}\right) /\left(Z_{p 2}+Z_{p 1}\right)$.

Being the most simple adaptor, it is direct to build the adaptor in a more practicable way, using the corresponding wave flow diagram in the Kelly-Lochbaum form [Markel and Gray, 1976] for both configurations (see Fig. 4.18).

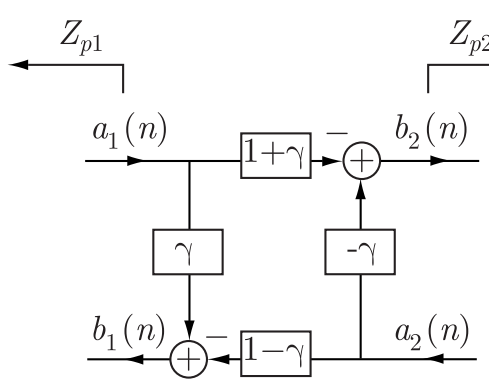

(a)
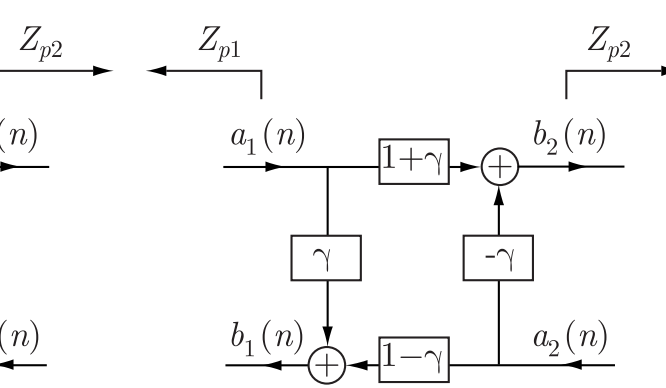

(b)

Figure 4.18. Wave flow diagram in Kelly-Lochbaum form of (a) serial and (b) parallel adaptor.

\subsection{Discussion}

In this chapter, it has been presented how, from the physical formulation of the sound propagation it is possible to build discrete-time methods able to simulate the wave propagation and their characteristic phenomena. This chapter deals with the mathematic fundamentals of some of these methods, particularly the Finite-Difference Time-Domain (FDTD) method and the Digital Waveguide Mesh (DWM) method.

The original FDTD method is based on a staggered distribution of the 
pressure and particle velocity components and their values are updated under the so-called leapfrog scheme. However, since the variable of interest in room acoustic simulation is the pressure, the FDTD method uses the wave equation in order to considerably reduce the computational cost and memory storage, and only pressure values are calculated in the algorithm. In each time step, each discretized pressure value is updated from the neighboring and previous discrete pressure values.

On the other hand, the DWM method decomposes the signal into incoming and outgoing pressure components. In a cartesian grid, these pressure components travel along characteristic directions. From neighboring pressure components, the pressure at each discretized point is updated as a linear combination of the incoming components.

It is possible to demonstrate how both methods are equivalent in homogeneous and inhomogeneous media. Although the results from simulating the same scenario with both methods are the same, different aspects such as efficiency and suitability for boundary condition definition distinguish them.

However, this equivalence allows the creation of hybrid models, where different areas of the whole mesh can be implemented with each one of the methods and it is possible to take advantages from each paradigm of these areas in particular.

The importance of these methods lies in the accuracy and the simplicity to implement the algorithms, having advantages over geometrical methods, since the sound wave propagation phenomena such as diffraction, interference, ... (except for the air absorption) appear without any extra effort in their implementation, assuring the accuracy of these methods for room acoustic purposes.

Finally, the Wave Digital Filters (WDF) are introduced, not as sound field simulation method, but also as a method for discretizing continuous system. They provide an approach to digital filter structures that are analogous to analog filter structures that have a certain desirable properties. These digital filters will have a fundamental role in next chapter. 


\section{Frequency-Dependent Boundary Conditions for Discrete-Time Methods}

The SOUND PROPAGATION IN ROOM ACOUSTICS IS THE CONSEQUENCE OF A BOUNDED MEDIUM, where the walls, ceiling and floor reflect part of the energy striking on them, as has been presented in Chap. 2. The resulting sound field is the addition of each one of the reflected waves appearing. Moreover, each reflected wave is reflected again in a different wall.

Summarizing, a particular room acoustic sound field (apart from position and characteristics of sources) is mainly the consequence of

- Room geometry: The position and number of the walls determine the number of reflections. Furthermore, the relative distance between source and walls, and also with the distance between walls, determines the amplitude and the phase of the reflected waves.

- Absorbing properties: In each one of the reflections, the reflected walls contains a fraction of the incident energy. These reflections are dependent of the wall material properties, being in the general case, frequency-dependent absorbing.

Then, the particular wall distribution and the material used in each one of the walls gives a characteristic sound field distribution [Kuttruff, 4th 
edition, 2000]. Therefore, the proper definition of the room in terms of the wall absorbing properties is fundamental to obtain accurate simulations.

The use of the discrete-time methods, apart from many other advantages (see Chap. 4), simplifies considerably the geometry definition in simulation: the characteristic regular discretization on these methods makes it easy to decide what cells do or do not belong to the boundaries, compared with other methods. However, to define impedance boundary conditions and particularly, the frequency-dependent one, is not a simple task with these methods, nor in the electromagnetic approach [Beggs et al., 1992, Maloney and Smith, 1992, Sullivan, 1992] neither in the acoustic field simulation [Özyörük and Long, 1997, Fung and Ju, 2004].

\subsection{Introduction}

In room acoustics, the reflections are mostly considered as either specular or diffuse (or a combination of both). As mentioned in Sec. 3.4.1, several solutions to diffuse surfaces have been successfully proposed [Murphy et al., 2008b]. However, specular reflections are not accurately modeled. For that reason, it is necessary to propose a method for including, in a simple and efficient way, realistic models of impedance on the simulation such as the locally reacting impedance model (see Sec. 2.4.1.3). Such a model would improve the accuracy of the whole acoustic simulation model. The locally reacting model is particularly important because it facilitates the modelling of specular reflections, since the impedance value is not dependent on the wave angle of arrival and it has a considerable advantage over models that take explicit account of the mechanical properties of the surface [Pierce, 1994, Kuttruff, 4th edition, 2000].

Those impedance boundary conditions in the discrete-time methods should require the following characteristics:

- Efficiency: the space discretization of a whole volume such as an ordinary room requires a huge amount of memory, and the sound field calculation over that mesh, has a considerable computational cost. It would be desirable if the boundary condition presence in a simulation did not excessively increase these computer resources.

- Accuracy: the model must accomplish the impedance physical law 
properties, i.e., in terms of frequency and angle of arrival.

- Stability: the model should be stable for the longest range of impedance boundary condition parameters, being desirable to be unconditionally stable.

This chapter deals with the problem of the impedance boundary conditions definition in the discrete-time methods, with special emphasis on the frequency-dependent case. In this chapter, contributions regarding the realization of boundary conditions in these numerical methods are presented together with several simulations demonstrating the suitability of these new algorithms. The chapter is mainly divided in two large sections: the first section deals with the boundary conditions in the DWM method, whereas the second deals with the FDTD method. In the DWM section (Sec. 5.2), a review of the current state-of-the-art of the boundary conditions is first presented. In Sec. 5.2.2, an analysis of the effect of choosing the reflection factor as a boundary condition is presented, and during Sec. 5.2.3 and Sec. 5.2.4 some new algorithms which solve the specular reflection in a DWM are introduced. In Sec. 5.3, a similar structure is followed for the FDTD method, with a review of the previous methods (see Sec. 5.3.1) and the introduction of a new method 5.3.2. In Sec. 5.4, although the scope of this thesis is not to validate physically the proposed algorithms, a first approach to assure the certainty of one of them is presented. Finally, the chapter is summarized.

\subsection{Boundary conditions in the DWM method}

In this section, a review of the state-of-the-art boundary conditions implementation in DWM methods is made, and an analysis will be presented of the limited accuracy of the current methods for frequency-dependent boundaries. After this, some solutions are proposed in order to define locally reacting impedances as boundary conditions in the DWM method.

\subsubsection{Previous approaches}

The first approach to the boundary condition in room acoustics for a DWM is presented by Savioja et al. [Savioja et al., 1996b], where the starting point appears in the definition of 1-D digital waveguide boundaries [Smith, 1992, 1996]. Let us consider the scattering (air) junctions at the edge of a 
boundary as in Fig. 5.1, where $p\left(\tilde{\mathbf{r}}_{B}, n\right)$, and this is connected to a "dummy" boundary scattering junction $p\left(\tilde{\mathbf{r}}_{D}, n\right)$, having a different admittance $Y_{B}$, from the admittance of the air $Y_{J}$. This "dummy" junction is useful to define the boundary condition since the change of admittance is the reason to produce a reflection $R$ defined through Eq. 2.70, but with an angle $\theta=$ 0 [Murphy and Beeson, 2007]:

$$
R=\frac{Y_{J}-Y_{B}}{Y_{J}+Y_{B}} .
$$

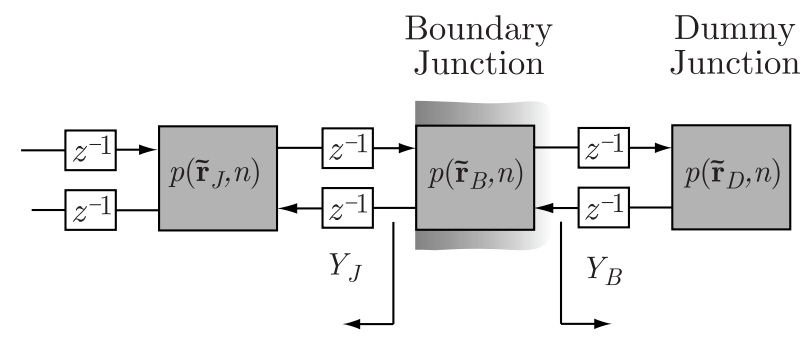

Figure 5.1. Interaction between a digital waveguide and boundary filters.

The boundary junction value is calculated as

$$
p\left(\tilde{\mathbf{r}}_{B}, n\right)=(1+R) p\left(\tilde{\mathbf{r}}_{J}, n-1\right)-R p\left(\tilde{\mathbf{r}}_{B}, n-2\right) .
$$

Although this method was originally proposed for the W-DWM, it is expressed in a more efficient way for the K-DWM, Eq. 5.2.

As an example, a 1-D simulation with a length $L$ is carried out with a $R=0.5$ on one side, and a perfectly reflecting boundary condition on the other side (Fig. 5.2). The simulation is presented each 100 time steps and displaced one $\mathrm{dB}$ in respect to the previous one. In these results, it can be observed how the wave component which strikes on the right side is returned with half of its energy.

It is important to note how this method only works for frequencyindependent boundary conditions (see Sec. 4.3), since the admittances of the scattering junction are defined as frequency-independent. This equation gives an exact solution for this 1-D case, except for the $R=0$ case, where 


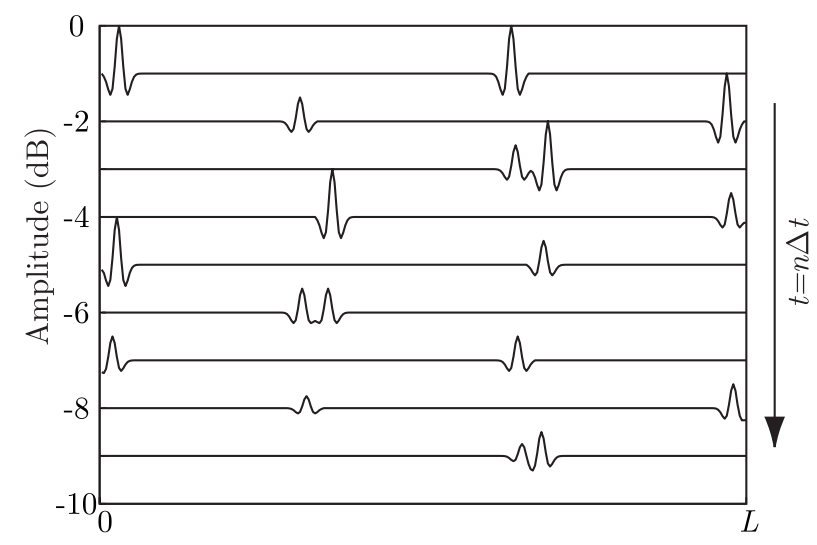

Figure 5.2. Example of a 1-D simulation with a constant reflection factor $R=0.5$ condition at position $x=0$ and hard wall at $x=L$. Each simulation represents the pressure solution at instant $+100 \Delta t$ and it has been displaced $-1 \mathrm{~dB}$.

some undesirable reflections appear. However, as referenced at Sec. 3.4.1, this is not a common handicap for the room acoustic simulation, except in cases where it is desirable to simulate the effects of open windows or doors; since this is not a common practice, no more approaches to this topic are made in this thesis.

Then, a logical solution to the multidimensional mesh problem is to directly adapt this solution, where $p\left(\tilde{\mathbf{r}}_{J}, n\right)$ is the scattering junction situated as a perpendicular neighbor [Savioja et al., 1999]. Since this method defines the boundary condition in terms of the impedance (or variation of the impedance), what is expected from this method works as a locally reacting impedance model. This has been analyzed in great detail by Kelloniemi et al. [Kelloniemi et al., 2004]. In this work, a detailed study of the angle behaviour has been made and improved through spatial filtering. This has also been adapted to improve nearly anechoic conditions in the K-DWM [Kelloniemi et al., 2005].

This scheme, based on a K-DWM, is valid only for constant impedances since the specific impedances defined on a DWM are non frequency-dependent. However, the use of a W-DWM allows the incorporation of frequencydependent boundary conditions [Huopaniemi et al., 1997]. It consists of 
the use of a time convolution of the boundary junction outgoing component $p_{B, r}^{-}(n)$ (or $\left.p_{r, B}^{+}(n)\right)$ by a digital filter representation of the reflection factor $r(n)(R(z)$ in the $z$-transformed domain), calculating the pressure at $p\left(\tilde{\mathbf{r}}_{B}, n\right)$ (see Fig. 5.3) as

$$
p\left(\tilde{\mathbf{r}}_{B}, n\right)=(\delta(n)+r(n)) * p_{B, r}^{-}(n),
$$

which is consistent with the definition of reflection factor (Eq. 2.65).

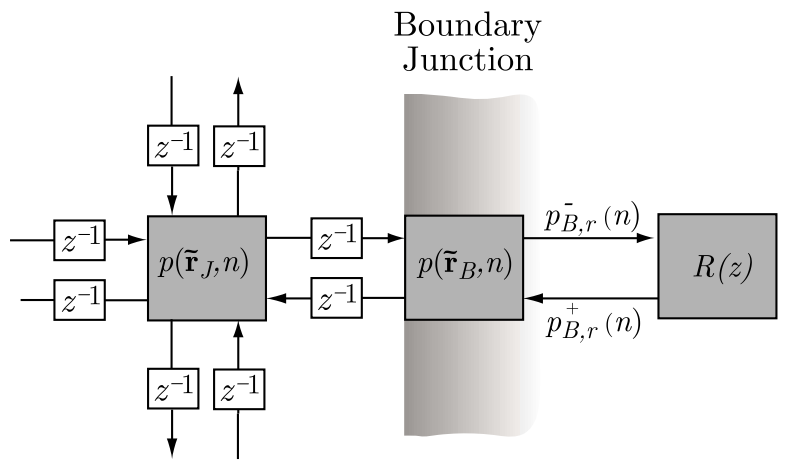

Figure 5.3. Interaction between DWM and frequencydependent boundary filters

For example, a 1-D simulation with a length $L$ is carried out with a frequency dependent reflection factor of a hard block layer of porous material impedance, given at Sec. 2.4.1.4. This reflection factor corresponds with an angle of incidence null, according to Eq. 2.69, from the analytical expression of the impedance. This boundary condition is situated at $x=0$ and the presentation scheme is the same as Fig. 5.4. In this case, a frequency-dependent boundary condition causes a modification not only in the energy of the striking pulse, but also in its original wave shape.

At this point, the work of Rochesso and Smith [Rochesso and Smith, 2003] should be mentioned, where a generalized Digital Waveguide Network is defined. This implies the definition of multivariable complex wave impedances, such as those deriving from multivariable lossy waveguides. This helps to build a mesh with different properties in the space, even with frequency dependent properties, implemented in practice using digital filters. However, these networks have not been tested in the locally reacting impedance sense. 


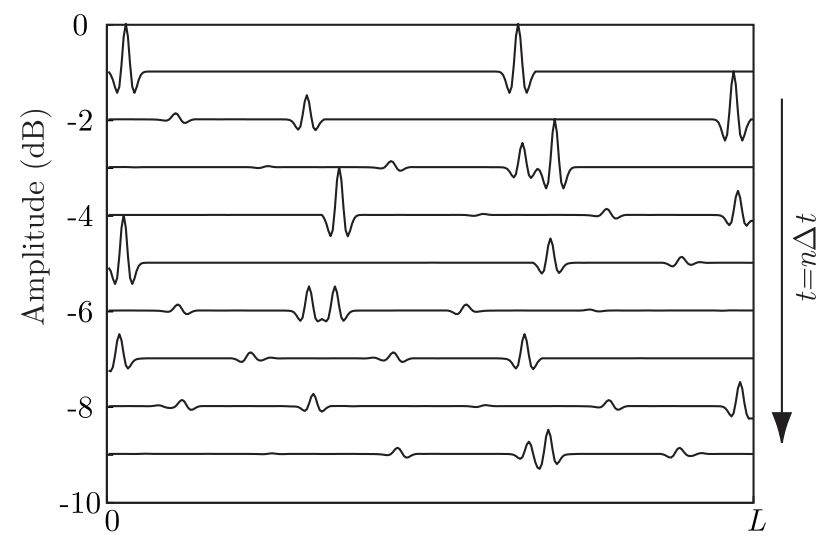

Figure 5.4. Example of a 1-D simulation with a frequency dependent reflection factor condition given by a hard-backed layer of porous material in one side. . Each simulation represents the pressure solution at instant $+100 \Delta t$ and it has been displaced $-1 d B$.

As has been seen in Sec. 4.5, this leads to the current tendency in discrete time-based room acoustics modeling, which is the use of hybrid models, where a K-DWM (or only pressure-dependent FDTD, see Sec. 4.2.3) is used to model the propagation (air) space and W-DWM is used to incorporate the boundary conditions [Savioja et al., 1994, Kelloniemi, 2006, Murphy et al., 2007], through a suitable adaptor between paradigms [Karjalainen and Erkut, 2004, Murphy and Beeson, 2007]. For this reason, the use of WDWM at the boundaries and a K-DWM in the space is a common practice, giving an efficient and versatile hybrid method (see Sec. 4.5).

Although this approach is very common and popular, many authors have noticed that a right correspondence of the use of the reflection factor as boundary condition does not exist with a locally reacting impedance model : non accurate specular reflection appears with this method in a multidimensional mesh [Murphy, 2000, Fontana, 2003]. The main reason for this discrepancy is based on the reflection factor digital filter: as detailed in Sec. 2.4.1.3, a locally reacting surface with a specific impedance of $Z(\omega)$ has a plane wave reflection factor that depends on the angle of incidence [Kuttruff, 4th edition, 2000], according to the Eq. 2.70. Since the angle of incidence $\theta$ is generally unknown, normal incidence is usually 
assumed [Huopaniemi et al., 1997]. In other words, with a given analytical impedance model (or with an impedance determined experimentally), the reflection factor filter is obtained by assuming that $\theta$ is zero. From now on, during this thesis, this method will be called Huopaniemi's method ${ }^{12}$.

The reflection factor filter used as a boundary condition has no knowledge about this angle. However, one can design a filter that takes into account the reflection, $R\left(\theta^{\prime}, \omega\right)$, in accordance with Eq. 2.70 for a certain angle of incidence, $\theta^{\prime}$. This angle acts as a parameter in the filter design. A more detailed analysis of the consequences using the reflection factor as a boundary condition is introduced during Sec. 5.2.2.

The contribution proposed by Kelloniemi [Kelloniemi, 2006] has to be emphasized, who has used an interpolated K-DWM mesh [Savioja and Välimäki, 2003] and the boundary conditions interact through a more efficient KW-conversor, based on [Karjalainen and Erkut, 2004]. This has really been the first application of the boundary conditions in a 3-D room acoustic simulation. However, same limitation is achieved when a reflection factor is used as a boundary condition, such as Huopaniemi's method.

In the next section (Sec. 5.2.2), an analysis of the consequences of using the reflection factor as a boundary condition in a multidimensional simulation is carried on.

\subsubsection{Physical interpretation of the use of the reflection factor as a boundary condition}

In this section, original results about the analysis of the reflection factor as boundary conditions in a W-DWM are presented [Escolano and Jacobsen, 2007]. The main concern of this section is to demonstrate if Huopaniemi's

\footnotetext{
${ }^{1}$ The authorship of this method is, depending on the consulted literature, from Huopaniemi or Savioja. Both are authors of [Huopaniemi et al., 1997], which is the first work where frequency-dependent boundary conditions for the W-DWM are presented. Previous work from this text, written by Savioja [Savioja et al., 1995, 1996a,b], has introduced the frequency-independent boundary condition; however, is a main concern of this thesis to deal with frequency-dependent conditions and the first author has been chosen to denominate the method in this thesis.

${ }^{2}$ In some texts, this method is called Digital Waveguide Filters [Fontana, 2003], however it is the personal conviction of the author of this thesis that this name does not necessarily represent the physical interpretation of the model, since a reflection filter is used as a boundary condition. As will be shown in Sec. 5.2.4, alternative methods can be used giving improved results and they could also be named as Digital Waveguide Filters.
} 
method corresponds with any physical impedance model, such as the locally reacting impedance model (Sec. 2.4.1.3).

In order to analyze the behaviour of the impedance model in a DWM, a semi-infinite impedance boundary surface is considered at $\tilde{\mathbf{r}}_{B}$, with the same structure such as the presented one in Fig. 5.4. The rest of the boundaries are assumed to be non-reflecting. Consider an incident broadband plane pressure wave $p_{i}(\mathbf{r}, t)$, and the corresponding particle velocity component $u_{x_{i}}(\mathbf{r}, t)=p_{i}(\mathbf{r}, t) \cos \theta /\left(\rho_{0} c\right)$.

If the impulse response of a reflection factor obtained in the simulation $\hat{R}(\theta, \omega)$ is defined as $\hat{r}(\theta, n)$ in the discrete-time domain, the reflected pressure is a time convolution, $p_{r}\left(\tilde{\mathbf{r}}_{B}, n\right)=p_{i}\left(\tilde{\mathbf{r}}_{B}, n\right) * \hat{r}(\theta, n)$. Information about the reflected component could be extracted from the finite difference method [Botteldooren, 1995].

Let us consider the finite difference formulation of the Euler equation of motion to calculate $u_{x}\left(\tilde{\mathbf{r}}_{B}, n\right)$ (see Eq. 4.14). However, the staggered nature of the FDTD method could be problematic to calculate the particle velocity in a DWM. For that reason, an approach using a backward finite difference is used to calculate the particle velocity component

$$
u_{x}\left(\tilde{\mathbf{r}}_{B}, n\right)=u_{x}\left(\tilde{\mathbf{r}}_{B}, n-1\right)-\frac{1}{\rho_{0}} \frac{\Delta t}{\Delta x}\left(p\left(\tilde{\mathbf{r}}_{B}, n\right)-p\left(\tilde{\mathbf{r}}_{B}-\Delta \tilde{\mathbf{x}}, n\right)\right) .
$$

By decomposing the pressure $p\left(\tilde{\mathbf{r}}_{B}, n\right)$ into incident and reflected components centered at $\tilde{\mathbf{r}}_{B}$, Eq. 5.4 becomes

$u_{x}\left(\tilde{\mathbf{r}}_{B}, n\right)=u_{x}\left(\tilde{\mathbf{r}}_{B}, n-1\right)-\frac{1}{\rho_{0}} \frac{\Delta t}{\Delta x}\left(p_{i}\left(\tilde{\mathbf{r}}_{B}, n\right) *(\delta(n)+\hat{r}(\theta, n))-p\left(\tilde{\mathbf{r}}_{B}-\Delta \tilde{\mathbf{x}}, n\right)\right)$,

where $\delta(n)$ is the delta Dirac function. This expression can easily be separated into direct and reflected parts (in the absence of reflection, only the incident part exists). In this case, it is the reflected part that is interesting,

$$
u_{x_{r}}\left(\tilde{\mathbf{r}}_{B}, n\right)=-\frac{1}{\rho_{0}} \frac{\Delta t}{\Delta x} p_{i}\left(\tilde{\mathbf{r}}_{B}, n\right) * \hat{r}(\theta, n) .
$$

The fraction $\Delta t / \Delta x$ can provide some information if certain algebraic modifications are made. Assuming equality in the Courant formula (Eq. 4.35), the fraction $\Delta t / \Delta x$ becomes 


$$
\frac{\Delta t}{\Delta x}=\frac{\frac{1}{\Delta x}}{c \sqrt{\left(\frac{1}{\Delta x}\right)^{2}+\left(\frac{1}{\Delta y}\right)^{2}}}=\frac{\Delta y}{c \sqrt{\Delta x^{2}+\Delta y^{2}}} .
$$

The fraction $\Delta y / \sqrt{\Delta x^{2}+\Delta y^{2}}$ can be seen to be identical with the cosine of the angle $\alpha$ that forms the diagonal direction with respect to the mesh coordinate system. After these algebraic transformations, Eq. 5.6 becomes

$$
u_{x_{r}}\left(\tilde{\mathbf{r}}_{B}, n\right)=-\frac{p_{i}\left(\tilde{\mathbf{r}}_{B}, n\right) * \hat{r}(\theta, n)}{\rho_{0} c} \cos \alpha .
$$

Note Eq. 5.8 is similar to the expected from the theory, $u_{x_{r}}\left(\tilde{\mathbf{r}}_{B}, n\right)=$ $-p\left(\tilde{\mathbf{r}}_{B}, n\right) * \hat{r}(\theta, n) \cos \theta /\left(\rho_{0} c\right)$; however, it is clear the dependence of the reflected component of the particle velocity does not depend on the angle of arrival and its value is constant with the angle of incidence. Taking into account the independence of the reflection factor filter with $\theta$, the result is consequent.

Finally, the total particle velocity component (incident plus reflected) in $x$-direction at $\tilde{\mathbf{r}}_{B}$ becomes

$$
u_{x}\left(\tilde{\mathbf{r}}_{B}, n\right)=\frac{p_{i}\left(\tilde{\mathbf{r}}_{B}, n\right)}{\rho_{0} c} \cos \theta-\frac{p_{i}\left(\tilde{\mathbf{r}}_{B}, n\right) * \hat{r}(\theta, n)}{\rho_{0} c} \cos \alpha .
$$

In order to determine the resulting reflection factor it is necessary to calculate the specific impedance (the ratio of the pressure to the particle velocity) at $\tilde{\mathbf{r}}_{B}$ in the Fourier transformed domain,

$$
Z(\omega)=\frac{P\left(\tilde{\mathbf{r}}_{B}, \omega\right)}{U_{x}\left(\tilde{\mathbf{r}}_{B}, \omega\right)}=\frac{P_{i}\left(\tilde{\mathbf{r}}_{B}, \omega\right)(1+\hat{R}(\theta, \omega))}{P_{i}\left(\tilde{\mathbf{r}}_{B}, \omega\right)\left(\frac{\cos \theta}{\rho_{0} c}-\frac{\hat{R}(\theta, \omega) \cos \alpha}{\rho_{0} c}\right)}
$$

Solving for the reflection factor $\hat{R}(\theta, \omega)$ of the DWM in Eq. 5.10 gives

$$
\hat{R}(\theta, \omega)=\frac{Z(\omega) \cos \theta-\rho_{0} c}{Z(\omega) \cos \alpha+\rho_{0} c} .
$$

However, the impedance $Z(\omega)$ realized by a filter based on Eq. 2.70 and a given value of $\theta^{\prime}$ is 


$$
Z(\omega)=\frac{\rho_{0} c}{\cos \theta^{\prime}} \frac{1+R\left(\theta^{\prime}, \omega\right)}{1-R\left(\theta^{\prime}, \omega\right)} .
$$

Finally, substituting Eq. 5.12 into Eq. 5.11, one obtains a relation between the reflection factor assumed in the filter design, $R\left(\theta^{\prime}, \omega\right)$, and the actual reflection factor obtained in the simulation, $\hat{R}(\theta, \omega)$,

$$
\hat{R}(\theta, \omega)=\frac{R\left(\theta^{\prime}, \omega\right)\left(\frac{\cos \theta}{\cos \theta^{\prime}}+1\right)+\left(\frac{\cos \theta}{\cos \theta^{\prime}}-1\right)}{R\left(\theta^{\prime}, \omega\right)\left(\frac{\cos \alpha}{\cos \theta^{\prime}}-1\right)+\left(\frac{\cos \alpha}{\cos \theta^{\prime}}+1\right)} .
$$

To summarise, Eq. 2.70 (also Eq. 5.12) is the analytical expression for the plane wave reflection factor, Eq. 5.11 is the reflection factor obtained by the DWM expressed in terms of the desired impedance, and Eq. 5.13 is the reflection factor obtained by the DWM expressed in terms of the actual angle of incidence $\theta$, the filter parameter $\theta^{\prime}$, and the diagonal angle in the mesh $\alpha$. Equation 5.13 shows that the best agreement is obtained when the angle of incidence, the angle selected in the filter design, and the diagonal angle in the mesh are the same (see Fig. 5.5 for details). Note, however, that $\alpha$ is determined by the mesh; on the other hand, at least the impedance model can be improved at some angles by using $\theta^{\prime}=\alpha$ (see Sec. 5.2.2.1). It can be concluded that according to the DWM impedance model (Eq. 5.11) the obtained reflection factor depends on the angle of sound incidence, but not in the same manner as a real locally reacting impedance surface does.

\subsubsection{Examples}

To examine the behavior of Huopaniemi's method in a W-DWM approximation respect to a locally reacting impedance, some implementations have been made. A 2-D rectangular mesh with $900 \times 900$ cells $(\Delta x=\Delta y)$ is designed with a boundary filter at $x=0$, and the other boundaries are absorbing boundary conditions [Murphy and Mullen, 2002]. The sampling frequency is $80 \mathrm{kHz}$, and the excitation is a Ricker wavelet pulse [Vetterli and Kovacevic, 1995] with a bandwidth of $20 \mathrm{kHz}$. These parameters have been selected so as to reduce the effects of dispersion in the DWM. The impedance is modeled as a hard-backed layer of porous material, which is described by Delany and Bazley's expressions [Delany and Bazley, 1970] and detailed in Sec. 2.4.1.4 for a $0.1 \mathrm{~m}$ layer of porous material with a flow resistivity of $1000 \mathrm{~kg} / \mathrm{m}^{3} / \mathrm{s}$. In this example, the reference spectrum responses used are the ones obtained directly from the theoretical reflection factor 


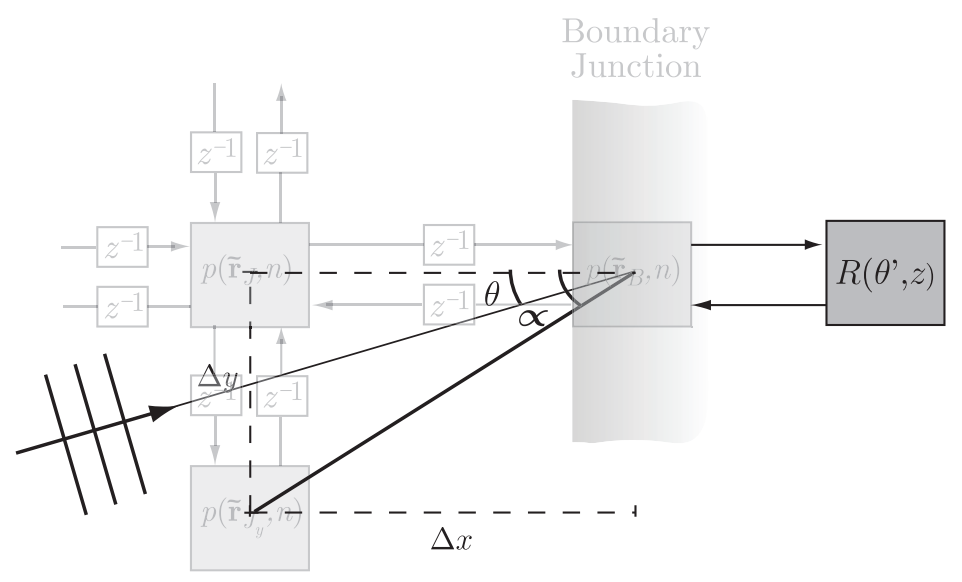

Figure 5.5. Structure and relation between the different angles involved in the boundary condition analysis in a DWM.

(see Fig. 2.6). The coefficients of the digital filter are defined according to Yule-Walker's algorithm with an IIR digital filter of 60th order using the analytical response of the reflection factor through Eq. 2.70.

As mentioned in the foregoing, the boundary filter can be designed with one degree of freedom, the parameter $\theta^{\prime}$. In the literature this parameter has invariably been selected as 0 ; in other words, the reflection factor that occurs for normal incidence has been used. However, as shown above, a better approximation to a locally reacting surface model is obtained, at least for some angles, by choosing $\theta^{\prime}=\alpha$, which in this case means $\alpha=\pi / 4$. In the particular case, when $\theta=\theta^{\prime}=\alpha$, the reflection factor measured is exactly the same as the designed filter. Both designs are presented in Fig. 5.6.

In order to determine the reflection factor in the mesh, both a point source that generates the sound field and an observation point are arranged such that the angle of incidence can be varied. The point source has been placed far enough from the boundary surface for the plane wave reflection factor to be indistinguishable from the spherical reflection factor [Butov, 1981]. The difference between the incident and reflected spectrum must be compensated for geometrical propagation losses; in the 2-D case this is $1 / \sqrt{r}$. 

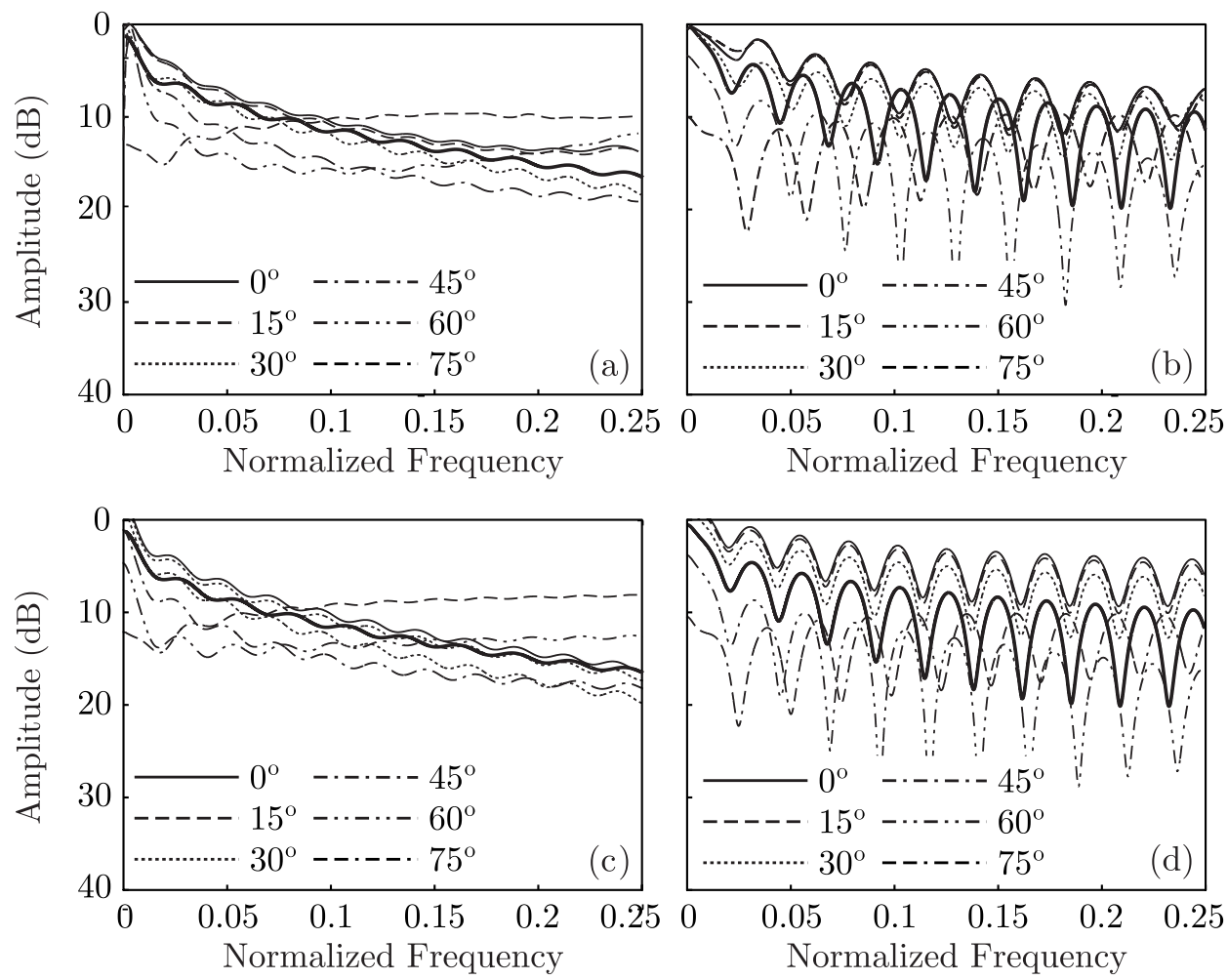

Figure 5.6. Measured reflection factor with (a) $\theta^{\prime}=0$ and (b) $\theta^{\prime}=\pi / 4$ for different angles of incidence. Predicted reflection factor according Eq. 5.13 with (c) $\theta^{\prime}=0$ and (d) $\theta^{\prime}=\pi / 4$. The solid wide line indicates the transfer function for the selected boundary condition $R\left(\theta^{\prime}, \omega\right)$. The solid wide line indicates the transfer function of the used filter.

Figure 5.6(a) shows different reflection factors obtained in a DWM when $\theta^{\prime}=0$. The figure demonstrates that the resulting reflection factors disagree with the reflection factor assumed in the filter design and there is an evident dependence with the angle of arrival; however, this dependence is far from the expected (see Fig. 2.6). For the different angles of incidence, a behavior similar to that of the filter can be observed: some similar distribution of ripples and their shape, but there is a different tendency for each one of the reflection factors to situate the average level of these ripples. In addition to these differences small frequency shifts can be discerned. These frequency 
shifts are not only due to the inherent (artificial) dispersion of the DWM algorithm, which is more evident at high frequencies (the strongest dispersion occurs for normal incidence), but also, there exist differences due to the method itself, as it can be observed at low frequencies. On the other hand, Fig. 5.6(c) shows the simulation obtained through Eq. 5.13: through simple inspection it is evident there exists a considerable agreement with measurements. Some differences appear from the dispersion, since this equation does not include it. However, the most important source of error comes from those reflection factor values which are near to the $0 \mathrm{~dB}$ and when $\theta \approx 0$; these misalignments can be observed in this simulation, giving as a result a reflection factor higher than $0 \mathrm{~dB}$, although they are lower than $3 \mathrm{~dB}$. What it is evident is that these values are not possible, since it would mean an amplification of total reflective walls of normal incident waves, and this is not possible. Despite these inaccuracies, the proposed model shows an important agreement with the measurements.

Figure 5.6(b) represents the case where $\theta^{\prime}=\pi / 4$. It can be seen that the differences between the results and the design for most angles of incidence are moderate except for the highest angle of incidence. Note that in the case of an angle incidence of $\theta=\pi / 4$, then $\hat{R}(\theta, \omega)=R\left(\theta^{\prime}, \omega\right)$, and this is confirmed by the results (see Eq. 5.13). Furthermore, it is well-known that the dispersion of the DWM method is minimized for the incidence angle $\alpha$ and then, the agreement with the expected is maximum. The predicted reflection factor under this design is also presented in Fig. 5.6(d). As in the previous example, there are evident similarities between the prediction model and the measurements, although same inaccuracies appear in this example as well; however, it should be emphasized these differences are due to frequency shifting, not due to the spectrum shape, which is nearly the same.

Both figures demonstrate that the general model for boundary conditions in the DWM method do not correspond to a locally reacting surface, although a reflection factor that depends on the angle of incidence occurs. The deviations from the behavior of a locally reacting impedance surface could be predicted from Eq. 5.13.

\subsubsection{Realization of a LRI model in a DWM based on angle detection}

According to Sec. 5.2.2, the main error caused by using the reflection factor as a boundary condition is a lack of plane wave angle of arrival information. 
A first approach to solve this problem could be to detect the angle of arrival. In the following contribution of this thesis, the intensity vector information is used to detect the plane wave angle of arrival. With this information, the right reflection factor filter is determined. However, as will shown later, this information is not enough to obtain the right filter: some modifications should be made.

\subsubsection{Angle detection}

Here a plane wave is considered as the incident wave in a 2-D space. The angle of incidence can be calculated from the $x$ - and $y$-components of the incident intensity vector (Eq. 2.49),

$$
\mathbf{I}(\mathbf{r}, \omega)=\frac{1}{2} \Re\left\{P(\mathbf{r}, \omega) \mathbf{U}^{*}(\mathbf{r}, \omega)\right\},
$$

where $P(\mathbf{r}, \omega)$ is the spectrum of the sound pressure and $\mathbf{U}(\mathbf{r}, \omega)$ is the spectrum of the particle velocity vector, as follows,

$$
\theta(\omega)=\arctan \left(\frac{I_{y}(\omega)}{I_{x}(\omega)}\right)
$$

A finite difference approximation to Euler's equation of motion, Eq. 4.14 and 4.15 , can be used for determining the particle velocity components [Botteldooren, 1995]. However, the total particle velocity is not the quantity of concern; it is the component associated with the incident wave that is required. In the W-DWM this can very easily be obtained because the sound pressure is naturally decomposed into incident and reflected components.

Consider a boundary surface parallel to the $y$-axis. The incident $x$ component of the particle velocity component, since $u_{B, r}^{-}(n)=u_{B, J}^{+}(n)$ according to Fig. 5.7, may be calculated as

$$
u_{B, r}^{-}(n)=u_{B, r}^{-}(n-1)-\frac{\Delta t}{\rho_{0} \Delta x}\left[p_{B, r}^{-}(n)-p_{J, B}^{-}(n)\right] .
$$

This pressure value is the one that travels from the mesh to the reflection digital filter through the delay line (see Fig. 4.4). Equation 5.16 could be seen as a finite difference Euler's equation with a backward difference to the temporal derivative, applied to incident components. 


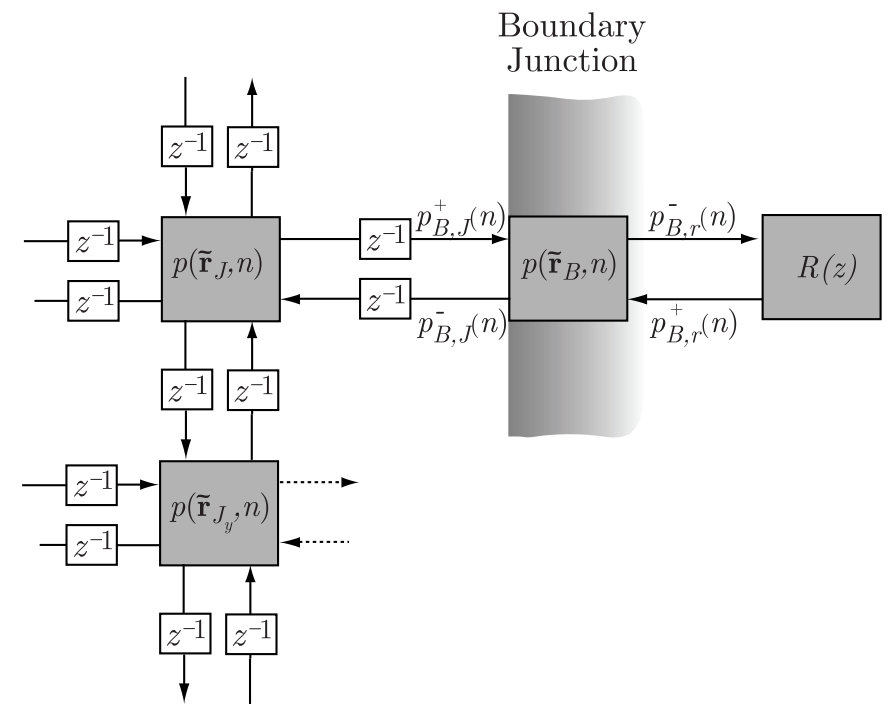

Figure 5.7. Scattering junction distribution used for the realization of a LRI based on angle detecion.

After checking Eq. 5.16, more simplicity could be expected if Eq. 4.54 is used; however, it is the author's experience that some errors can be found if a point source is not far enough to be considered as plane wave and the plane wave assumption of Eq. 4.54. For that reason, it works better to use Eq. 5.16 since no wave shape is assumed. The proof of Eq. 5.16 is in checking the plane wave case. When a plane wave arrives at this position, Eq. 5.16 becomes Eq. 4.55. This is possible to demonstrate, if a plane wave is considered, after some algebraic modifications as follows:

After using Eq. 4.59, Eq. 5.16 yields

$$
u_{B, r}^{-}(n)=u_{B, r}^{-}(n-1)-\frac{\Delta t}{\rho_{0} \Delta x}\left[p_{B, r}^{-}(n)-p\left(\tilde{\mathbf{r}}_{J}, n\right)+p_{J, B}^{+}(n)\right],
$$

and by using Eq. 4.60, it could be determined

$$
p_{J, B}^{+}(n)=p_{B, J}^{-}(n)=p_{B, r}^{+}(n-1) .
$$

Inserting Eq. 5.18 into Eq. 5.17, and now grouping terms in Eq. 5.17, 
it yields

$$
u_{B, r}^{-}(n)=\left[u_{B, r}^{-}(n-1)-\frac{\Delta t}{\rho_{0} \Delta x} p_{B, r}^{+}(n-1)\right]-\frac{\Delta t}{\rho_{0} \Delta x}\left[p_{B, r}^{-}(n)-p\left(\tilde{\mathbf{r}}_{J}, n\right)\right]
$$

In Sec. 5.2.2, it was shown how the delay line impedance is calculated as $Z=\sqrt{2} \rho_{0} c=\rho_{0} \Delta x / \Delta t$. Then,

$$
u_{B, r}^{-}(n-1)-\frac{\Delta t}{\rho_{0} \Delta x} p_{B, r}^{+}(n-1)=u_{B, r}^{-}(n-1)-u_{B, r}^{+}(n-1)=u_{\overrightarrow{B, r}}\left(\tilde{\mathbf{r}}_{B}, n-1\right)
$$

the equation is rearranged as

$$
u_{B, r}^{-}(n)=u \underset{B, r}{\longrightarrow}\left(\tilde{\mathbf{r}}_{B}, n-1\right)-\frac{\Delta t}{\rho_{0} \Delta x}\left[p_{B, r}^{-}(n)-p\left(\tilde{\mathbf{r}}_{J}, n\right)\right]
$$

Finally, if $p_{B, r}^{-}(n)=p\left(\tilde{\mathbf{r}}_{B}, n\right)-p_{B, r}^{+}(n)$ is considered, what it is obtained is

$$
u_{B, r}^{-}(n)=\left[u_{\overrightarrow{B, r}}\left(\tilde{\mathbf{r}}_{B}, n-1\right)-\frac{\Delta t}{\rho_{0} \Delta x}\left[p\left(\tilde{\mathbf{r}}_{B}, n\right)-p\left(\tilde{\mathbf{r}}_{J}, n\right)\right]\right]-\frac{\Delta t}{\rho_{0} \Delta x} p_{B, r}^{+}(n),
$$

and this equation is equivalent to write

$$
u_{B, r}^{-}(n)=u_{B, r}^{\underset{B}{\longrightarrow}}\left(\tilde{\mathbf{r}}_{B}, n\right)-u_{B, r}^{+}(n),
$$

demonstrating the suitability of the proposed method to calculate the incident component of the particle velocity for plane waves. It should be noted how this is possible to define since the interaction with the boundary condition is done through a 1-D link. Regarding the particle velocity component parallel to the surface, the total component is a straightforward calculation for the junction.

On the other side, since there exists a 1-D connection between the mesh, there is no link between the scattering junction situated inside the 
boundary, $\tilde{\mathbf{r}}_{B}$, in the $y$-direction (e.g., in the parallel direction to the wall, see Fig. 5.7). One may think about to calculate the $y$-component by using the gradient between consecutive boundary junctions in that direction; however, it requires to know the total pressure at those junction and they depend on reflected component at the same time. The easiest way is to assume this component could be calculated from the gradient between scattering junction in $\tilde{\mathbf{r}}_{J}$ and $\tilde{\mathbf{r}}_{J_{y}}$. It should be taken into account that this component does not change after reflection.

Let us note now Eq. 5.15 requires working in the frequency domain; in principle each frequency component might arrive with a different angle. The approach here is to use a window of $N$ samples at each boundary scattering junction $\tilde{\mathbf{r}}_{B}$ and for each sound field component, such as pressure and particle velocity components. It should be noted that the data can be modified with the usual windows (e.g. Hamming or Hann).

In each time step the window is shifted one sample; $\tau$ indicates the discrete temporal displacement of the window. Applying a the Fourier transform to each window, $P\left(\tilde{\mathbf{r}}_{B}, \omega, \tau\right), U_{B, r}^{-}\left(\tilde{\mathbf{r}}_{B}, \omega, \tau\right)$ and $U_{\overrightarrow{J, J_{y}}}\left(\tilde{\mathbf{r}}_{B}, \omega, \tau\right)$ are obtained, where $U_{B, r}^{-}$is the incident component in $x$-direction. Note that this approach corresponds to applying a short-time Fourier transform [Proakis and Manolakis, 1998] with a window of $N$ components and an overlap of $N-1$ terms over the sound field components. This procedure provides a spectrum of the different components for each time step, with a frequency resolution related to $N$.

According to Eq. 5.15, the two components of the intensity are used to obtain the angle of incidence at each position on the boundary $\tilde{\mathbf{r}}_{B}$,

$$
\theta\left(\tilde{\mathbf{r}}_{B}, \omega, \tau\right) \simeq \arctan \left(\frac{\Re\left\{P\left(\tilde{\mathbf{r}}_{B}, \omega, \tau\right) U_{\overrightarrow{J, J}}^{*}\left(\tilde{\mathbf{r}}_{J}, \omega, \tau\right)\right\}}{\Re\left\{P\left(\tilde{\mathbf{r}}_{B}, \omega, \tau\right) U_{B, r}^{-*}\left(\tilde{\mathbf{r}}_{B}, \omega, \tau\right)\right\}}\right) .
$$

For each displacement $\tau$ of the time window, the results make it possible to determine the reflection factor for each frequency according to Eq. 2.70. Figure 5.9 shows examples of spectrograms with different angles of incidence. The data of each shift register have been windowed with a Hamming window. At each position of the spectrogram an arrow indicates the intensity vector.

Once the angle $\theta$ is obtained, the reflection factor filter is obtained ac- 


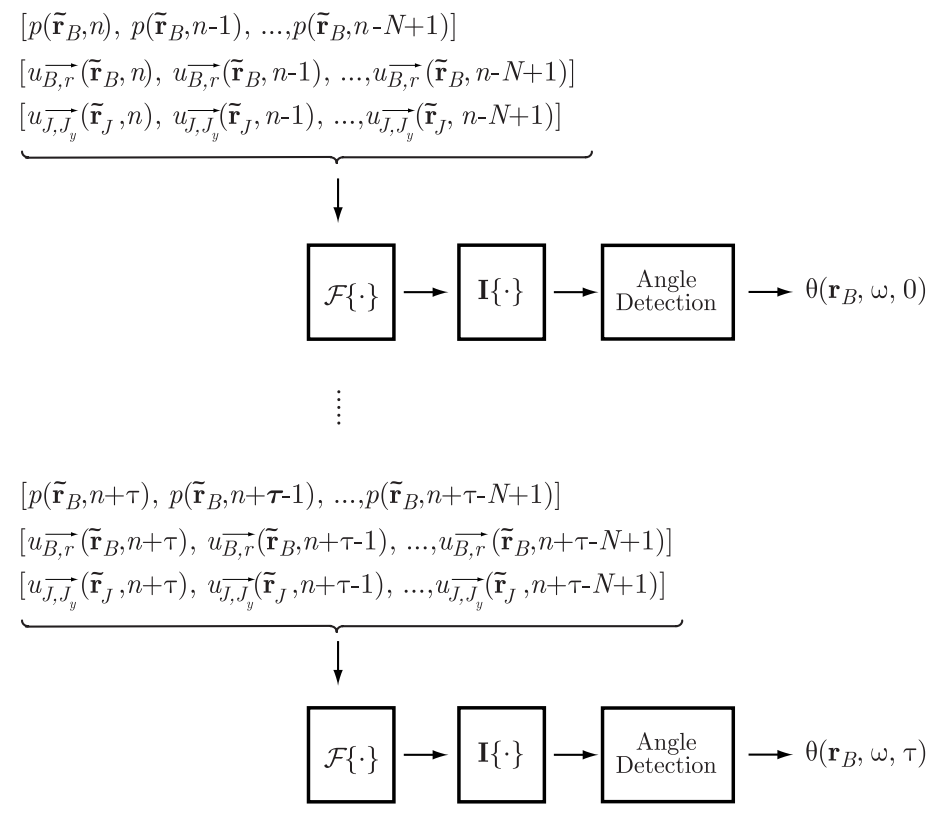

(a)

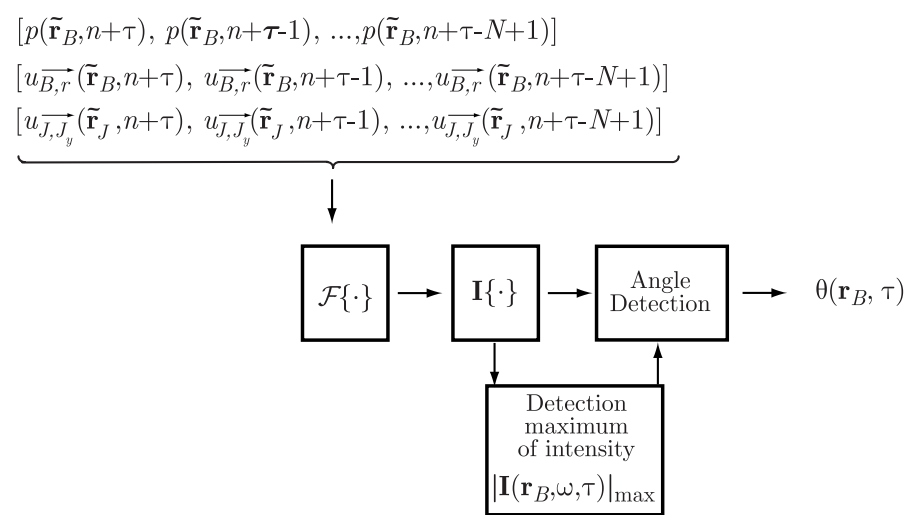

(b)

Figure 5.8. Schemes used for angle detection: (a) for each single frequency; (b) based on the estimation of maximum value of the absolute value of the intensity vector. 
cording Eq. 2.69 at each $\tau$ step. The method described in the foregoing can be expected to give good results. However, this solution will be extremely computationally expensive because a digital filter representing the analogue reflection factor is determined at each time step and each boundary point (see Fig. 5.8(a)). A more efficient solution should be found.

Consider a plane wave that strikes the boundary with the same angle of incidence at all frequencies. This case is not unrealistic, because the first reflections in a room correspond to a set of waves with the frequency content of the source impinging on the walls. In this proposed model, it is assumed that $\theta\left(\tilde{\mathbf{r}}_{B}, \omega, n\right) \simeq \theta\left(\tilde{\mathbf{r}}_{B}, n\right)$, and this angle is selected from the frequency at which the maximum absolute value of the intensity, $\left|\mathbf{I}\left(\tilde{\mathbf{r}}_{B}, \omega, \tau\right)\right|_{\max }$, occurs in each time step for (see Fig. 5.8(b)). This modification makes it possible to define a reflection factor for each angle of incidence before the simulation (off-line) and to apply it at each time step. As can be observed in Fig. 5.9, this assumption is consistent with plane waves, where the vector arrows are nearly the same at all frequencies. This algorithm can be improved if discrete angles are considered $(\theta=k \Delta \theta, \forall k \in \mathbb{N})$, and then a discrete number of filters would be required.

\subsubsection{Filter compensation}

It has been demonstrated in Sec. 5.2.2 that there is a difference between the reflection factor assumed in the filter design, $R\left(\theta^{\prime}, \omega\right)$, being $\theta^{\prime}$ and the one obtained in the simulation, $\hat{R}(\theta, \omega)$, where $\theta$ is the "true" angle of incidence, when Huopaniemi's method is used. This difference can be calculated analytically from Eq. 5.13.

Since the method previously outlined makes it possible to estimate $\theta$ one can design the filter accordingly, that is, $\theta^{\prime}=\theta$. Equation 5.13 now becomes

$$
\hat{R}(\theta, \omega)=\frac{2 R(\theta, \omega)}{R(\theta, \omega)\left(\frac{\cos \alpha}{\cos \theta}-1\right)+\left(\frac{\cos \alpha}{\cos \theta}+1\right)} .
$$

It is apparent that even when information about the angle of incidence is available and used in the filter design there are differences between the analytical theory and the result. However, this effect can be avoided by redesigning the boundary filter such that the resulting reflection factor becomes identical to the desired analytical expression, that is, $\hat{R}(\theta, \omega)=R_{\text {ana }}(\theta, \omega)$, which gives 


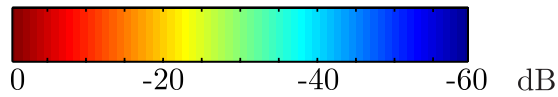

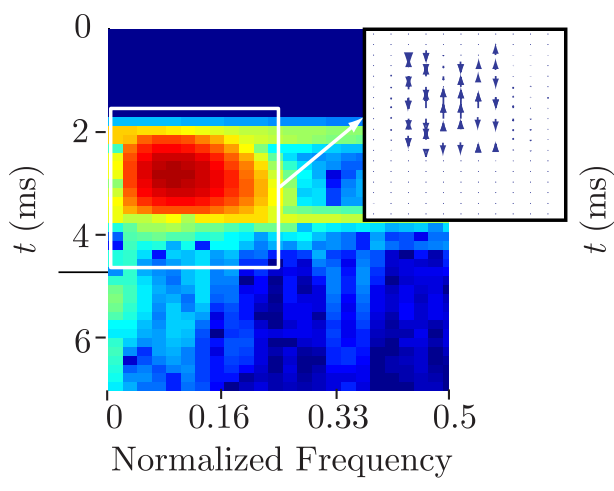

(a)

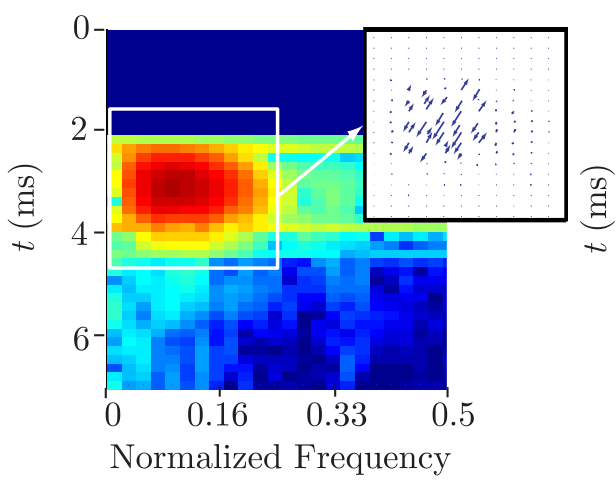

(c)

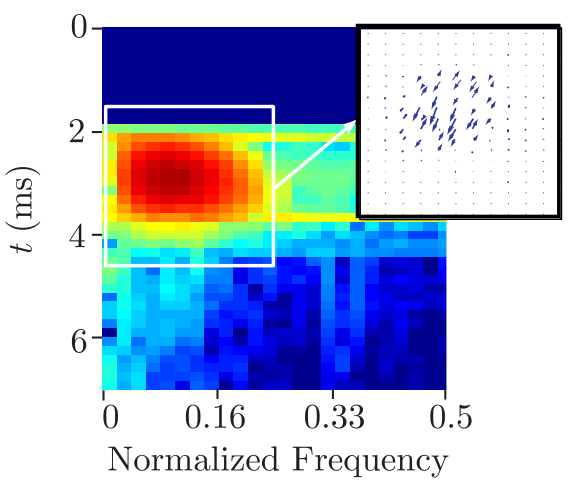

(b)

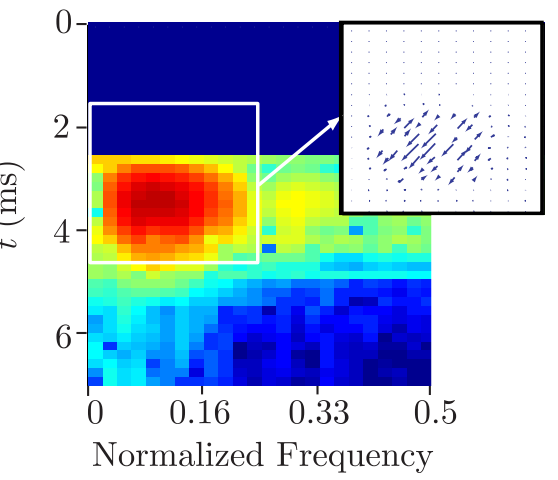

(d)

Figure 5.9. Spectrograms of different angles of incidence: a) 0 degrees, b) 15 degrees, c) 30 degrees and d) 45 degrees.

$$
R(\theta, \omega)=\frac{R_{\mathrm{ana}}(\theta, \omega)\left(\frac{\cos \alpha}{\cos \theta}+1\right)}{2-R_{\mathrm{ana}}(\theta, \omega)\left(\frac{\cos \alpha}{\cos \theta}-1\right)}
$$

In other words, if the boundary filter is designed according to Eq. 5.26 where $R_{\text {ana }}$ is given by Eq. 2.70 then the resulting reflection factor actually behaves as the analytical expression. 


\subsubsection{Examples}

In order to examine the accuracy of the proposed model, a 2-D simulation study has been performed. The mesh consists of $1500 \times 1500$ cells, where the boundary condition simulating a frequency-dependent impedance is implemented on one side, and the remaining boundaries are considered absorbing [Murphy and Mullen, 2002]. The size of the cells is determined from the Courant formula (Eq. 4.36). The sampling frequency is $80 \mathrm{kHz}$, and the represented bandwidth correspond to a normalized frequency of $0.25 \times f_{s}$ [Duyne and Smith, 1993]. The measured reflection factors are obtained again as the ratio of the spectrum of the reflected signal to the spectrum of the incident plane wave for different angles of incidence. The set-up of these example are mostly equal to the one appeared in Sec. 5.2.2.1.

The impedance boundary condition is in accordance with the example in Sec. 2.4.1.4. The coefficients of the digital filter are determined using Yule-Walker's algorithm with an IIR digital filter of 60th order using the analytical response of the analytical reflection factor (see Fig. 2.6). Note that the impedance spectrum obtained by the digital filter design should be as accurate as possible. Even small differences between the reference impedance and the digital filter design can give rise to significant differences between the physical (or theoretical) reflection factor and the one obtained by the digital impedance. The analysis windows are Hamming and they are based on 128 samples.

Figure 5.10 compares the analytical reflection factor with the results of the simulation for various angles of incidence (0, 15, 30 and 45 degrees), both without and with the suggested compensation method. As can be seen the compensation reduces the difference between the desired and the obtained reflection factor significantly. As expected, compensated and uncompensated results are identical for this particular case when $\theta=\alpha$, cf. Eq. 5.13.

Regarding the size of the windows, the larger the size, the higher the computational cost. However, note that high resolution is not needed, because only the maximum value of the intensity, $\left|\mathbf{I}\left(\tilde{\mathbf{r}}_{B}, \omega, \tau\right)\right|_{\max }$, is used.

However it should be mentioned that the suggested method only works in cases where a single angle of incidence is enough to describe the reflection. 

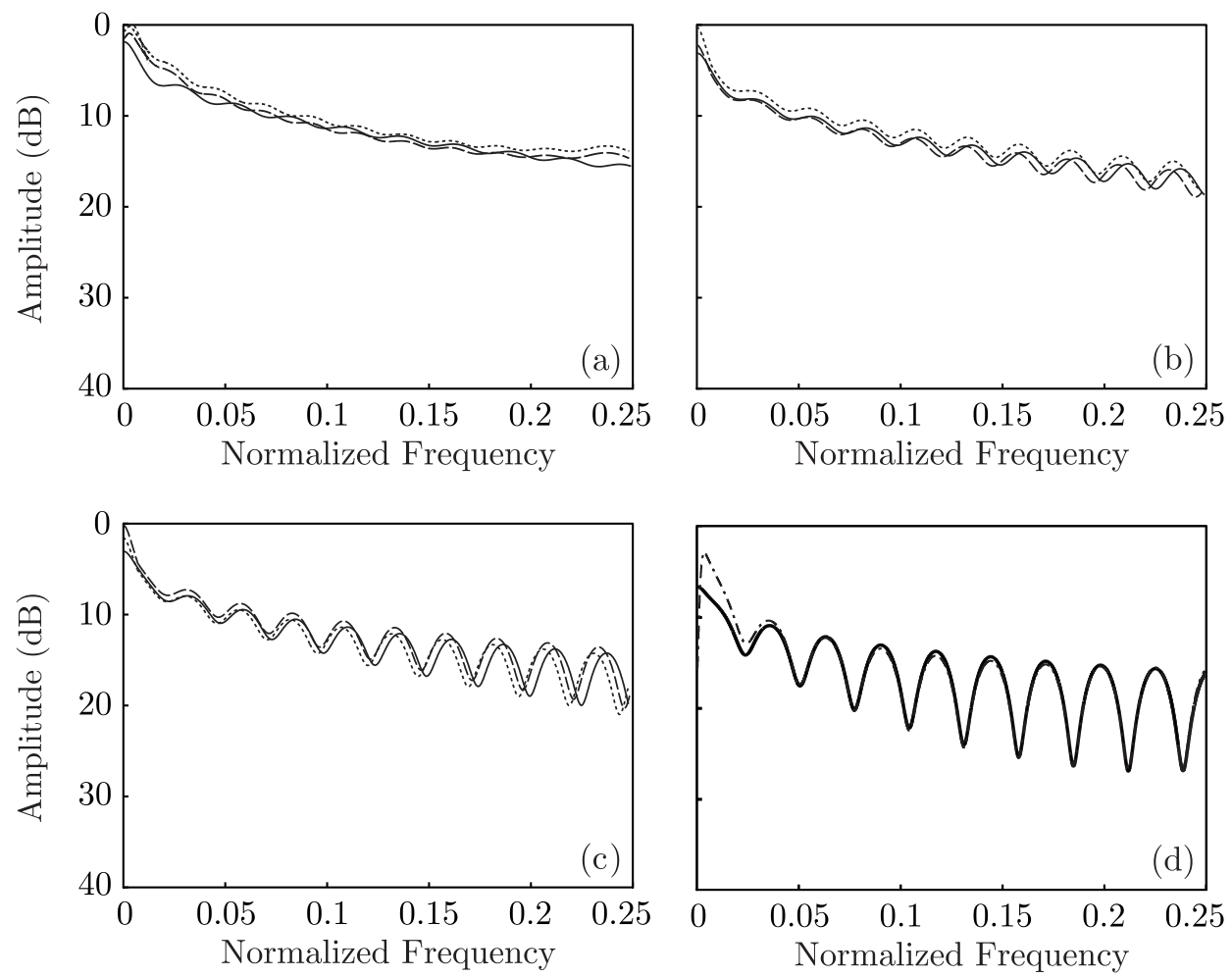

Figure 5.10. Reflection factors obtained without filter compensation (dotted line) and with filter compensation (dashed line) compared with analytical results (solid line) for a) $\theta=0$ degrees, b) $\theta=15$ degrees, c) $\theta=30$ degrees and d) $\theta=45$ degrees.

\subsubsection{Limitations of the method}

The main advantage of this method lies in the possibility of improving the accuracy of the results when Huopaniemi's method is used. Since the main handicap of this method lies in the lack of information of the angle of arrival, a simple method for detecting this angle is proposed.

The results show a good agreement with the expected ones and it makes this angle of arrival independent in each scattering junction. However, it should be said there are a set of serious limitations for a practical implementation in a simulation system: 
- It only works when only one plane wave arrives in each one of the boundary junctions at the same time. In case several waves with different angles of arrival strike on the surfaces, each scattering junction will process the information according to the total particle velocity component as a linear combination of those arriving waves.

- It requires a constant update of the filter coefficients of each filter in accordance with the detected angle of arrival. This considerably increases the computational cost. Furthermore, time-varying digital filters can provide errors and distortions occurring during parameter transitions [Mourjopoulos et al., 1990].

- The angular dispersion distorts the particle velocity components and thus, it can lead to errors in the angle detection. These errors can be especially problematic for those reflection factors which, for a given impedance, are very sensitive to changes in this angle.

- The main handicap lies in the assumption of the plane wave incident component as the outgoing component for a particular scattering junction. As has been said in Sec. 4.3.1. Direct identification in the multidimensional case for the plane wave components and the outgoing/incoming variables does not exist. However, the reason for making this equivalence in this algorithm is due to the fact that the boundary junction is connected through a 1-D junction and it has been calculated through the gradient of the outgoing components and not directly with the outgoing particle velocity components. It has been probed how this approach works for detecting the angle of arrival for a plane wave with only an incident component.

Although it could be thought the angle detection could be a direct improvement for Huopaniemi's method, due to these presented reasons, this makes it very difficult to extend the algorithm in a real room acoustic simulation. Thus, it is necessary to find a new algorithm able to considerably reduce the computational cost and increase the accuracy of the boundary conditions. 


\subsubsection{Realization of a LRI model in a DWM based on mixing modeling strategies}

Since the modification of Huopaniemi's method is neither accurate in most senses nor efficient, it is necessary to find another point of view to solve the problem. According to Sec. 5.2.2, the main problem with this method in a multidimensional mesh lies in the non-physical boundary description, since a reflection factor is used to determine the portion of reflected energy without taking into account the angle of arrival.

However, in Sec. 2.4.1.3, it was seen how in a locally reacting impedance, the impedance is independent of the plane wave angle of arrival. Therefore, it seems logical that using the impedance to describe a boundary condition, the reflecting waves would have a description according to Sec. 2.4.1.3. However, so far, this has not been done yet, since only pressure or incoming/outgoing components are involved in a DWM method.

Therefore, a combination of different methods may be the solution. Thus, the proposed method is based on the interaction between different paradigms: the impedance is modeled with a Wave Digital Filter (WDF) [Fettweis, 1986] (see Sec. 4.7 for details) and with the proper interaction model, it is coupled to the sound field space, a Digital Waveguide Mesh. In this section, a review of the employed mixing modelling strategy is presented. In the next subsection, the proper model of the impedance is introduced and finally, the whole system is analyzed, demonstrating how the proposed realization is able to simulate a locally reacting impedance.

\subsubsection{Mixing modeling strategy}

The mixing modeling strategy is a recent trend in digital sound synthesis by physical modeling, using different paradigms to simulate various parts of musical instruments [de Sanctis et al., 2003, Karjalainen et al., 2003, Rabenstein et al., 2007]. Although in room acoustics this tendency is not usual, i.e., just by mixing both K- (at the mesh itself) and W-DWM (at the boundaries) using KW-converters to mix both methods [Karjalainen, 2004, Murphy and Beeson, 2007] could be seen as an example of this mixing modeling.

The interaction between the different parts assume continuous transitions from one side to the other, and is no fracture and discontinuity at the models interface appear. This seamless transition exists when all derivatives 
below a certain number ${ }^{3}$, are equivalent at both sides of the interface [Petrausch, 2007].

Based on this concept, this contribution addresses the coupling of a DWM method with a digital filter representing the impedance, rather than the reflection factor. The mixing modeling strategy for this coupling is the one proposed by Petrausch and Rabenstein [Petrausch and Rabenstein, 2004, 2007]. They propose the use of WDFs ports as a common interface between different blocks.

WDFs appear as a discretization process of complex systems, where each network element is discretized separately by a bilinear transformation [Proakis and Manolakis, 1998]. The main reason for using WDFs as a common interface is the capacity to avoid potential computational problems, e.g., delay-free loops or potentially unstable implicit equations in the interconnection of the network elements [Szczupak and Mitra, 1975]. This can be done by using the so-called wave variables. Originally, Fettweis [Fettweis, 1986] defined these variables as voltage-current (see Sec. 4.7.1), but considering the impedance analogy [Beranek, 1954] they can be used as acoustic variables.

In the adaptation it has to be taken into account this forces wave variables to have a directional component. Then, it is convenient to express WDF variables in a vectorial way

$$
\begin{aligned}
\mathbf{a}(\tilde{\mathbf{r}}, n)^{T} & =\mathbf{I} p(\tilde{\mathbf{r}}, n)+Z_{p} \mathbf{u}(\tilde{\mathbf{r}}, n)^{T}, \\
\mathbf{b}(\tilde{\mathbf{r}}, n)^{T} & =\mathbf{I} p(\tilde{\mathbf{r}}, n)-Z_{p} \mathbf{u}(\tilde{\mathbf{r}}, n)^{T},
\end{aligned}
$$

where $\mathbf{I}=[1,1]^{T}$ is all-ones vector. The variables $\mathbf{a}(\tilde{\mathbf{r}}, n)$ and $\mathbf{b}(\tilde{\mathbf{r}}, n)$ represent the outgoing and incoming wave variables, respectively, and propagation information is inherent in them at position $\tilde{\mathbf{r}}$, since each vector component of these wave variables depends linearly on one of the particle velocity components. That means $\mathbf{a}(\tilde{\mathbf{r}}, n)=\left[a_{x}(\tilde{\mathbf{r}}, n), a_{y}(\tilde{\mathbf{r}}, n), a_{z}(\tilde{\mathbf{r}}, n)\right]$ and $\mathbf{b}=\left[b_{x}(\tilde{\mathbf{r}}, n), b_{y}(\tilde{\mathbf{r}}, n), b_{z}(\tilde{\mathbf{r}}, n)\right]$ and these are vectors with dimensions as the pressure. In the multi-dimensional case, $Z_{p}$ does not correspond with the physical characteristic impedance of the medium; it must be calculated in order to achieve this wave decomposition, as will be shown later.

\footnotetext{
${ }^{3}$ This number is calculated as $\min \left(n_{1}, n_{2}\right)$, where $n_{1}$ and $n_{2}$ are the maximum order of the spatial derivative of each one solutions of the models involved into the entire model.
} 
In case just one component is used, it results as

$$
\begin{aligned}
a_{n}\left(\tilde{\mathbf{r}}_{B}, n\right) & =p(\tilde{\mathbf{r}}, n)+Z_{p} u_{n}(\tilde{\mathbf{r}}, n) \\
b_{n}\left(\tilde{\mathbf{r}}_{B}, n\right) & =p(\tilde{\mathbf{r}}, n)-Z_{p} u_{n}(\tilde{\mathbf{r}}, n) .
\end{aligned}
$$

The subindex $n$ indicates that wave variables have the same direction as the component of the particle velocity $u_{n}(\tilde{\mathbf{r}}, n)=\mathbf{u}(\tilde{\mathbf{r}}, n) \cdot \hat{\mathbf{n}}$. In the same way, the Kirchhoff variables, $p$ and $u_{n}$ can be expressed in terms of the wave variables as

$$
\begin{aligned}
p(\tilde{\mathbf{r}}, n) & =\frac{a_{n}(\tilde{\mathbf{r}}, n)+b_{n}(\tilde{\mathbf{r}}, n)}{2}, \\
u_{n}(\tilde{\mathbf{r}}, n) & =\frac{a_{n}(\tilde{\mathbf{r}}, n)-b_{n}(\tilde{\mathbf{r}}, n)}{2 Z_{p}} .
\end{aligned}
$$

The proper choice of the port resistance $Z_{p}$ makes it possible to separate the design of the block elements from the definition of their interaction by appropriate adaptor elements [Petrausch et al., 2005a]. Here, the nomenclature $Z_{p}$ is used instead the classic $R$ for the resistance in order to not create confusion with the reflection factor. The separation and interaction in WDF terms ensures an unconditionally stable model provided if both parts, the DWM method of the sound field and the digital filter realization of the impedance, are independently stable. Once delay-free loops are removed, the discrete-time domain system can be computed iteratively.

\subsubsection{Impedance model}

As stated above, the coupling between digital filters representing the impedance with WDF ports in this study has been realized using a certain model [Petrausch and Rabenstein, 2004]. This solution is based on a State Space Systems (SSS) approach, which states that any linear system can be expressed in terms of an SSS.

In the present case, let us consider a filter $Z(z)$, where $z$ is the $z$ transform variable, a single input, $u_{n}(\tilde{\mathbf{r}}, n)$, and a single output $p(\tilde{\mathbf{r}}, n)$ are related as follows, 


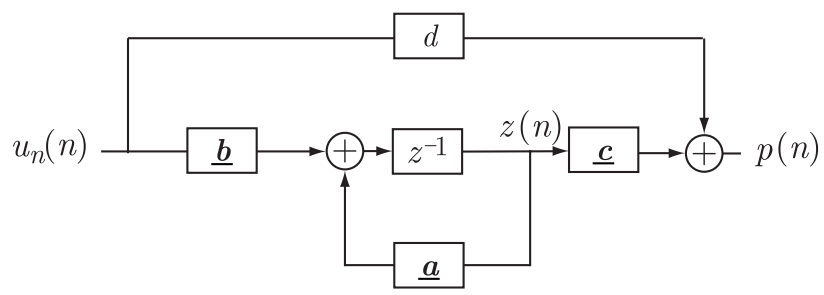

(a)

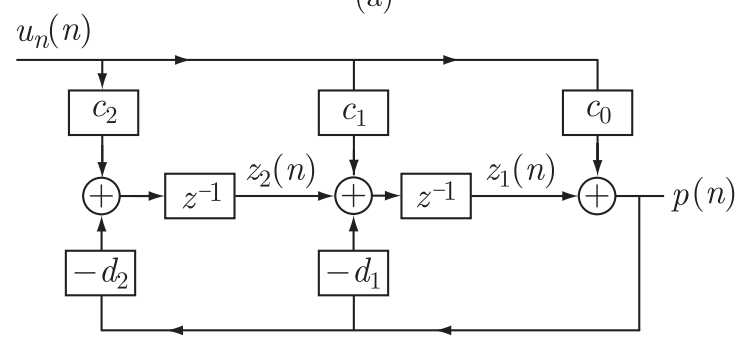

(b)

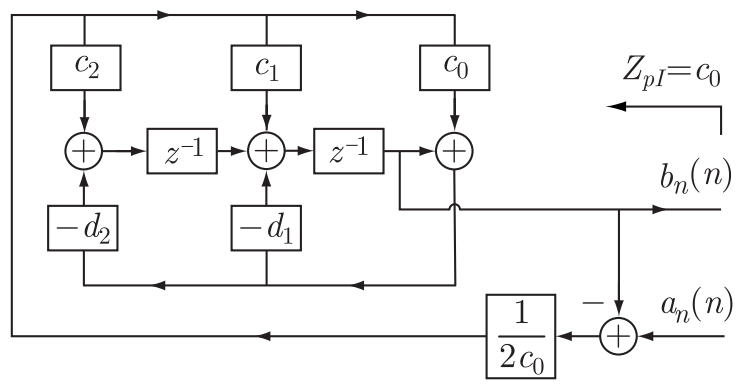

(c)

Figure 5.11. (b) Digital Filter scheme representing a certain impedance. (c) Interaction of a second order IIR filter with wave digital filter ports.

$$
\begin{aligned}
\mathbf{z}(\tilde{\mathbf{r}}, n+1) & =\underline{\mathbf{a z}}(\tilde{\mathbf{r}}, n)+\underline{\mathbf{b}} u_{n}(\tilde{\mathbf{r}}, n), \\
p(\tilde{\mathbf{r}}, n) & =\underline{\mathbf{c z}}(\tilde{\mathbf{r}}, n)+d u_{n}(\tilde{\mathbf{r}}, n) .
\end{aligned}
$$

The vector $\mathbf{z}(n)$ is the system state. For this situation, $\underline{\mathbf{a}}$ is a matrix, $\underline{\mathbf{b}}$ and $\underline{\mathbf{c}}$ are vectors, and $d$ is a scalar (see [Proakis and Manolakis, 1998] and Fig. 5.11(a) for more details). 
Delay-free loops in the interconnection of the SSS with WDF are avoided by the following procedure: Eq. 5.34 is inserted into Eqs. 5.29 and 5.30, and then is forced to $Z_{p}=d$ (see [Petrausch and Rabenstein, 2004] for details). Then, $b(n)$ becomes independent of $u_{n}(n)$, avoiding instantaneous feedback and sources of instability. Thus,

$$
\begin{aligned}
u_{n}(\tilde{\mathbf{r}}, n) & =\frac{1}{2 d}\left(a_{n}(\tilde{\mathbf{r}}, n)-\underline{\mathbf{c z}}(\tilde{\mathbf{r}}, n)\right), \\
b_{n}(\tilde{\mathbf{r}}, n) & =\underline{\mathbf{c z}}(\tilde{\mathbf{r}}, n) .
\end{aligned}
$$

As an example, a second order IIR filter in a canonical observable form that represents the impedance is adapted to WDF ports. According to state space theory, a second order IIR digital filter is represented as an SSS by the equations

$$
\begin{aligned}
\mathbf{z}(\tilde{\mathbf{r}}, n+1) & =\left(\begin{array}{ll}
-d_{1} & 1 \\
-d_{2} & 0
\end{array}\right) \mathbf{z}(\tilde{\mathbf{r}}, n)+\left(\begin{array}{l}
c_{1}-c_{0} d_{1} \\
c_{2}-c_{1} d_{2}
\end{array}\right) u_{n}(\tilde{\mathbf{r}}, n) \\
p(\tilde{\mathbf{r}}, n) & =\left(\begin{array}{ll}
1 & 0
\end{array}\right) \mathbf{z}(\tilde{\mathbf{r}}, n)+c_{0} \cdot u_{n}(\tilde{\mathbf{r}}, n) .
\end{aligned}
$$

The digital filter that represents the impedance expressed in terms of WDF ports is shown in Fig. 5.11(c). Note that the specific impedance of the medium is always the independent term of the filter numerator polynomial, $c_{0}$.

Since these variables do not necessarily appear explicit in a scattering junction of a mesh, let us define the upper-index $f$ in order to indicate that $p^{f}(\tilde{\mathbf{r}}, n)$ and $u_{n}^{f}(\tilde{\mathbf{r}}, n)$ are the output and input of the digital filter at position $\tilde{\mathbf{r}}$.

\subsubsection{Model description}

In this section, the complete system is presented. The parts forming the whole system are explained in detail. Then, an analysis of the complete system will demonstrate its locally reacting impedance behaviour.

The most convenient and straightforward way to consider the behaviour at the boundary of a mesh structure is to set a boundary junction as having one other neighbour [Murphy, 2000]. This can be done by connecting a junction specifically situated just in the interface of the boundary itself, 


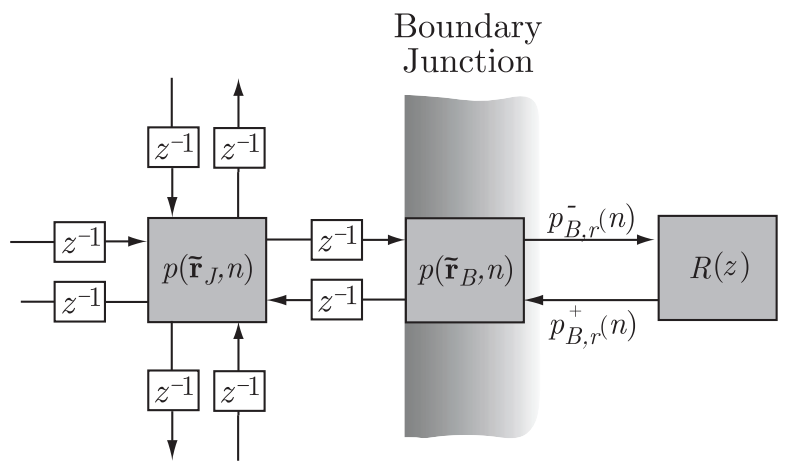

(a)

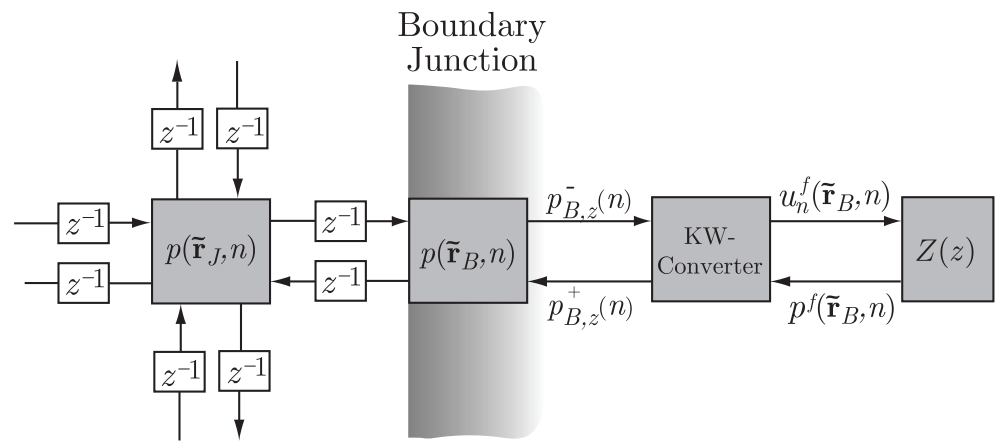

(b)

Figure 5.12. (a) Termination of a waveguide mesh due to the boundary resulting in a reflection, where the boundary condition is expressed in terms of the reflectance $R$. (b) Scheme of the proposed model, where the boundary condition is expressed in terms of the impedance $Z$.

$\tilde{\mathbf{r}}_{B}$ [Savioja et al., 1996b]. However, so far, the boundary condition has been expressed in terms of a reflection factor [Escolano and Jacobsen, 2007], where a constant angle of incidence is always assumed (see Fig. 5.12(a)); the presented method proposes expressing it in terms of the impedance function itself (see Fig. 5.12(b)), which is constant for any angle of incidence. Below, a frequency dependent impedance is assumed, giving a generalization of the boundary problem.

However, this interaction between the DWM and the impedance filter 
$Z(z)$ cannot consist of a direct connection for two main reasons: the first is related to the difference in the nature of the variables of each part; whereas the DWM is based on wave variables (there is a causal relationship between the incoming and outgoing wave components), the impedance is in terms of Kirchhoff variables (the impedance is defined as a ratio of two physical and observable variables). The second reason is the one mentioned in Sec. 5.2.4.1, where the main source of instability in the coupling of two digital system is the delay-free loops. Fortunately, both handicaps are solved using a common interaction in terms of WDF.

It has to be noted how, in the proposed method, the DWM see the boundary condition as reflection factor as well, since the entrance to the boundary are wave variables. However, the main difference lies in the boundary condition definition, where the proposed method implicitly takes into account the angle of arrival, since the normal particle velocity component to the boundary is the entrance to the filter, whereas the method of Fig. 5.12(a) assumes a certain unique angle for defining the reflection factor filter, giving a non physical model (see Sec. 5.2.2).

At this point, the complete model is introduced in Fig. 5.13 at a boundary junction $\tilde{\mathbf{r}}_{B}$. Once the conversion of the impedance filter in WDF ports has been solved in Sec. 5.2.4.2, the DWM variables have to be converted as well. In the part of Fig. 5.13, labeled as DWM-WDF converter, this process is carried out. This is done by just identifying the DWM variables with WDF variables through a simple scaling factor [Karjalainen, 2003, 2004]:

$$
\begin{aligned}
& p_{B, z}^{+}(n)=\frac{a\left(\tilde{\mathbf{r}}_{B}, n\right)}{2}, \\
& p_{B, z}^{-}(n)=\frac{b\left(\tilde{\mathbf{r}}_{B}, n\right)}{2},
\end{aligned}
$$

where $p_{B, z}^{+}(n)$ and $p_{B, z}^{+}(n)$ are the incoming and outgoing components traveling from the position $\tilde{\mathbf{r}}_{B}$ towards the impedance filter $Z(z)^{4}$.

Once both parts of the system are converted to WDF variables, it must be taken into account that each part has a different port resistance. This means that both parts cannot be directly connected. In order to couple these

\footnotetext{
${ }^{4}$ The incoming and outgoing wave directions will be defined from that scattering junction. Therefore, one has to assume there could have some changes in the wave variables definitions when they are compared with adaptors (see Sec. 4.7.3).
} 


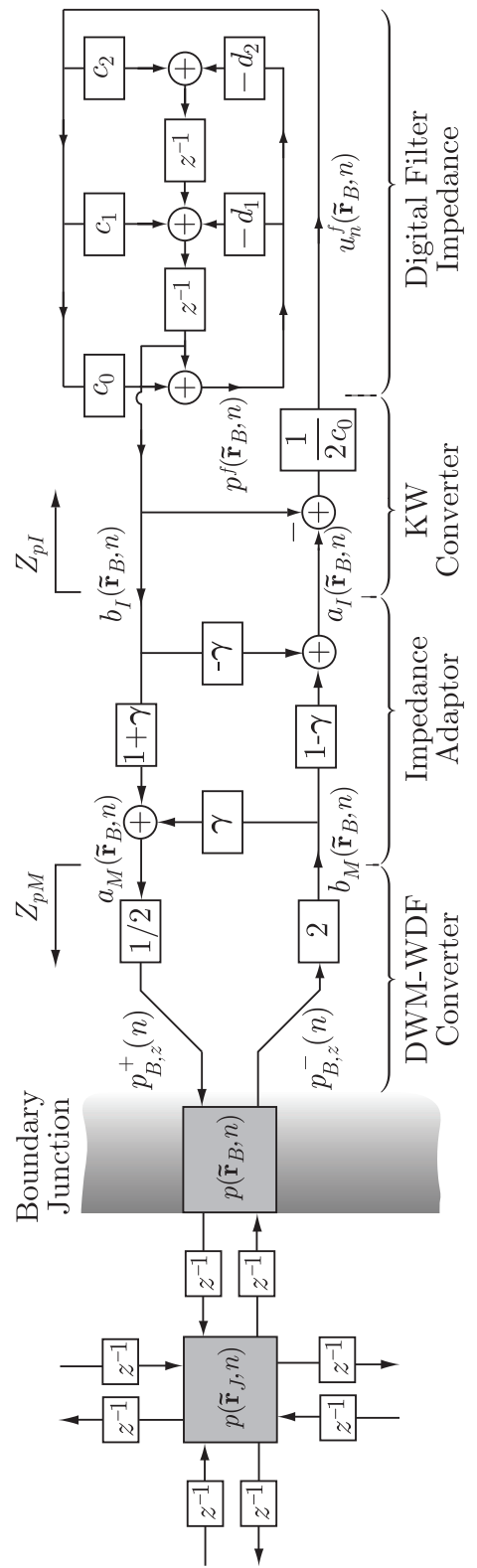

Figure 5.13. The complete proposed system. The DWM is connected with a digital filter realization of the impedance through a variables adaptation, in order to avoid instabilities. 
two WDFs and avoid instabilities, it is necessary to use a two-port adaptor (labeled as impedance adaptor in Fig. 5.13) to adapt both WDF impedances and avoid direct feedback loops. This adaptor, known as parallel adaptor in WDF literature [Bilbao, 2001], relates wave variables to different impedance ports (see Sec. 4.7.3 for details). A matrix description for a parallel adaptor of two WDF ports, $M$ (subindex for the mesh) and $I$ (subindex for the impedance), is

$$
\left(\begin{array}{c}
a_{M}\left(\tilde{\mathbf{r}}_{B, z}, n\right) \\
a_{I}\left(\tilde{\mathbf{r}}_{B, z}, n\right)
\end{array}\right)=\left(\begin{array}{cc}
\gamma & 1-\gamma \\
1+\gamma & -\gamma
\end{array}\right)\left(\begin{array}{c}
b_{M}\left(\tilde{\mathbf{r}}_{B, z}, n\right) \\
b_{I}\left(\tilde{\mathbf{r}}_{B, z}, n\right)
\end{array}\right)
$$

where $\gamma=\left(Z_{p I}-Z_{p M}\right) /\left(Z_{p I}+Z_{p M}\right)$.

In the particular case of the specific impedance of the mesh, $Z_{p M}$, it can be identified as the specific impedance of the medium $\rho_{0} c_{n}$, where $c_{n}$ is the (numerical) speed of sound in the mesh. It must be considered that the speed of sound in the mesh is not necessarily the same as the theoretical speed of sound $c$. Assuming a homogenous discretization of the mesh $(\Delta x=$ $\Delta y$ ), the speed of sound in the mesh can be calculated as $c_{n}=\Delta x / \Delta t$. In accordance with the Courant condition (see Eq. 4.35) and assuming this condition as equality, after some simple algebraic manipulations, the specific impedance of the DWM is

$$
Z_{p M}=\rho_{0} c \sqrt{2} .
$$

It has to be noted how this equivalence is true and constant in a frequency free-dispersion range. It has been assumed that this algorithm is performed on up to a quarter of the sampling frequency, as stated in Sec. 4.3.1, where the numerical speed of sound can be considered constant.

With the convenient arrangement of those cited elements, the whole mesh with a boundary condition is presented in Fig. 5.13. The boundary condition is connected to the DWM through a dummy junction. However, in this case, the DWM variables that connect this dummy junction to the impedance is converted to WDF variables through a simple scaling factor. These WDF ports are adapted in order to avoid reflections due to the port impedances mismatch. Once the WDF ports have adapted their impedance, they are conveniently filtered by a digital filter that represents the local impedance. 


\subsubsection{Analysis of the system}

In this section, the system presented in Sec. 5.2.4.3 is being analyzed in detail to demonstrate analytically that this model (Fig. 5.13) works as a locally reacting impedance. This analysis is carried out in two directions: from the DWM variables to the digital filter and vice versa.

For the purpose of this analysis, let us consider the following example: a second order IIR digital filter modeling a discrete point $\tilde{\mathbf{r}}_{B}$ of a plane surface with a normal vector in the $\hat{\mathbf{n}}$-direction and an impedance with impulse response $z\left(\tilde{\mathbf{r}}_{B}, n\right)$. Let us define $a_{M}\left(\tilde{\mathbf{r}}_{B}, n\right)$ and $b_{M}\left(\tilde{\mathbf{r}}_{B}, n\right)$ as the outgoing and incoming WDF variables obtained from the dummy junction to wave variables, according to Eqs. 5.38 and 5.39. These variables have a specific impedance $Z_{p M}$. Then, they are adapted to the WDF specific impedance of the digital filter $Z_{p I}$ [Petrausch and Rabenstein, 2004], through the adapter (see Eq. 5.40). Then, a more suitable transformation of the adaptor equation should be made, where the WDF port variables in the impedance $\left(a_{I}\left(\tilde{\mathbf{r}}_{B}, n\right)\right.$ and $\left.b_{I}\left(\tilde{\mathbf{r}}_{B}, n\right)\right)$ are obtained in terms of the WDF port variables in the mesh at position $\tilde{\mathbf{r}}_{B}$. With some simple algebraic manipulations, such wave variables become

$$
\begin{aligned}
& a_{I}\left(\tilde{\mathbf{r}}_{B}, n\right)=\alpha_{M}^{-} \cdot a_{M}\left(\tilde{\mathbf{r}}_{B}, n\right)+\alpha_{M}^{+} \cdot b_{M}\left(\tilde{\mathbf{r}}_{B}, n\right), \\
& b_{I}\left(\tilde{\mathbf{r}}_{B}, n\right)=\alpha_{M}^{+} \cdot a_{M}\left(\tilde{\mathbf{r}}_{B}, n\right)+\alpha_{M}^{-} \cdot b_{M}\left(\tilde{\mathbf{r}}_{B}, n\right),
\end{aligned}
$$

where $\alpha_{M}^{+}=\left(Z_{p M}+Z_{p I}\right) /\left(2 Z_{p M}\right)$ and $\alpha_{M}^{-}=\left(Z_{p M}-Z_{p I}\right) /\left(2 Z_{p M}\right)$

Inserting Eqs. 5.38 and 5.39 into Eqs. 5.42 and 5.43, WDF variable at the filter entrance can be obtained as a function of the DWM variable of the dummy junction

$$
\begin{aligned}
& a_{I}\left(\tilde{\mathbf{r}}_{B}, n\right)=\left(p_{B}^{+}\left(\tilde{\mathbf{r}}_{B}, n\right)+p_{B}^{-}\left(\tilde{\mathbf{r}}_{B}, n\right)\right)+\frac{Z_{p I}}{Z_{p M}}\left(p_{B}^{-}\left(\tilde{\mathbf{r}}_{B}, n\right)-p_{B}^{+}\left(\tilde{\mathbf{r}}_{B}, n\right)\right)(5.4 \\
& b_{I}\left(\tilde{\mathbf{r}}_{B}, n\right)=\left(p_{B}^{+}\left(\tilde{\mathbf{r}}_{B}, n\right)+p_{B}^{-}\left(\tilde{\mathbf{r}}_{B}, n\right)\right)-\frac{Z_{p I}}{Z_{p M}}\left(p_{B}^{-}\left(\tilde{\mathbf{r}}_{B}, n\right)-p_{B}^{+}\left(\tilde{\mathbf{r}}_{B}, n\right)\right)(.5
\end{aligned}
$$

Finally, from Eq. 5.44 and 5.45, and in accordance with Fig. 5.13 and Eq. 5.32, the input of the filter is calculated as 


$$
\frac{a_{I}\left(\tilde{\mathbf{r}}_{B}, n\right)-b_{I}\left(\tilde{\mathbf{r}}_{B}, n\right)}{2 Z_{p I}}=\frac{p_{B, z}^{-}(n)-p_{B, z}^{+}(n)}{Z_{p M}} .
$$

In accordance with the equivalence of the plane waves in a DWM stated in Sec. 4.3.1, and particularly, with Eq. 4.62, the entrance in the digital filter matches with the $\hat{\mathbf{n}}$-component of the particle velocity, which contains inherent information regarding the angle of arrival $\theta$

$$
\frac{a_{I}\left(\tilde{\mathbf{r}}_{B}, n\right)-b_{I}\left(\tilde{\mathbf{r}}_{B}, n\right)}{2 Z_{p I}}=\frac{p_{i}\left(\tilde{\mathbf{r}}_{B}, n\right)-p_{r}\left(\tilde{\mathbf{r}}_{B}, n\right)}{\rho_{0} c} \cos \theta,
$$

where $p_{i}\left(\tilde{\mathbf{r}}_{B}, n\right)$ and $p_{r}\left(\tilde{\mathbf{r}}_{B}, n\right)$ are the incident and reflected plane wave components (see Sec. 2.4.1.3).

It should be considered that Eq. 4.62 can be applied to the dummy junction without any change in its formulation, since only the normal component of the wall can be applied to the dummy junction, being consequent with Eq. 5.46.

Now, the next step is to demonstrate that the output of the filter $p^{f}\left(\tilde{\mathbf{r}}_{B}, n\right)$ is directly the pressure at the junction $p\left(\tilde{\mathbf{r}}_{B}, n\right)$. According to Fig. 5.13 and WDF theory, and taking into account $Z_{p I}=c_{0}$ (see Sec. 5.2.4.2):

$$
\begin{array}{r}
a_{I}\left(\tilde{\mathbf{r}}_{B}, n\right)=p^{f}\left(\tilde{\mathbf{r}}_{B}, n\right)+Z_{p I} \cdot u_{n}^{f}\left(\tilde{\mathbf{r}}_{B}, n\right), \\
b_{I}\left(\tilde{\mathbf{r}}_{B}, n\right)=p^{f}\left(\tilde{\mathbf{r}}_{B}, n\right)-Z_{p I} \cdot u_{n}^{f}\left(\tilde{\mathbf{r}}_{B}, n\right) .
\end{array}
$$

The inverse of Eqs. 5.42 and 5.43 can be useful for calculating the WDF ports in the mesh, $a_{M}\left(\tilde{\mathbf{r}}_{B}, n\right)$ and $\left.b_{M}\left(\tilde{\mathbf{r}}_{B}, n\right)\right)$ :

$$
\begin{aligned}
& a_{M}\left(\tilde{\mathbf{r}}_{B}, n\right)=\alpha_{I}^{-} \cdot a_{I}\left(\tilde{\mathbf{r}}_{B}, n\right)+\alpha_{I}^{+} \cdot b_{I}\left(\tilde{\mathbf{r}}_{B}, n\right), \\
& b_{M}\left(\tilde{\mathbf{r}}_{B}, n\right)=\alpha_{I}^{+} \cdot a_{I}\left(\tilde{\mathbf{r}}_{B}, n\right)+\alpha_{I}^{-} \cdot b_{I}\left(\tilde{\mathbf{r}}_{B}, n\right),
\end{aligned}
$$

where $\alpha_{I}^{+}=\left(Z_{p I}+Z_{p M}\right) /\left(2 Z_{p I}\right)$ and $\alpha_{I}^{-}=\left(Z_{p I}-Z_{p M}\right) /\left(2 Z_{p I}\right)$.

Inserting Eqs. (5.48) and (5.49) into Eqs. (5.50) and (5.51), the following equations are obtained: 


$$
\begin{aligned}
a_{M}\left(\tilde{\mathbf{r}}_{B}, n\right) & =p^{f}\left(\tilde{\mathbf{r}}_{B}, n\right)+Z_{p M} \cdot u_{n}^{f}\left(\tilde{\mathbf{r}}_{B}, n\right), \\
b_{M}\left(\tilde{\mathbf{r}}_{B}, n\right) & =p^{f}\left(\tilde{\mathbf{r}}_{B}, n\right)-Z_{p M} \cdot u_{n}^{f}\left(\tilde{\mathbf{r}}_{B}, n\right) .
\end{aligned}
$$

Once these equations are converted into DWM variables through the scaling factors, they become:

$$
\begin{aligned}
p_{B, z}^{-}(n) & =\frac{p^{f}\left(\tilde{\mathbf{r}}_{B}, n\right)+Z_{p M} u_{n}^{f}\left(\tilde{\mathbf{r}}_{B}, n\right)}{2}, \\
p_{B, z}^{+}(n) & =\frac{p^{f}\left(\tilde{\mathbf{r}}_{B}, n\right)-Z_{p M} u_{n}^{f}\left(\tilde{\mathbf{r}}_{B}, n\right)}{2} .
\end{aligned}
$$

Then, these variables are related to the pressure $p_{B}(n)$ just by adding both

$$
p\left(\tilde{\mathbf{r}}_{B}, n\right)=p_{B, z}^{+}(n)+p_{B, z}^{-}(n)=p^{f}\left(\tilde{\mathbf{r}}_{B}, n\right) .
$$

An alternative way to demonstrate the validity of the model is to calculate the reflection factor in the boundary scattering junction. Taking into account that the impedance is, in general, expressed in the frequency domain, the uppercase variables are used to denote the amplitude corresponding to a particular frequency $\omega$. This can be done using the impedance definition in this scattering junction $Z\left(\tilde{\mathbf{r}}_{B}, \omega\right)$ and Eqs. 5.52 and 5.53

$$
Z\left(\tilde{\mathbf{r}}_{B}, \omega\right)=\frac{P\left(\tilde{\mathbf{r}}_{B}, \omega\right)}{U_{n}\left(\tilde{\mathbf{r}}_{B}, \omega\right)}=Z_{p M} \frac{A_{M}\left(\tilde{\mathbf{r}}_{B}, \omega\right)+B_{M}\left(\tilde{\mathbf{r}}_{B}, \omega\right)}{A_{M}\left(\tilde{\mathbf{r}}_{B}, \omega\right)-B_{M}\left(\tilde{\mathbf{r}}_{B}, \omega\right)} .
$$

Through the adaptor equations 5.50 and 5.51, the impedance can be expressed in terms of the WDF ports in the impedance filter

$$
Z_{B}\left(\tilde{\mathbf{r}}_{B}, \omega\right)=Z_{p I} \frac{A_{I}\left(\tilde{\mathbf{r}}_{B}, \omega\right)+B_{I}\left(\tilde{\mathbf{r}}_{B}, \omega\right)}{A_{I}\left(\tilde{\mathbf{r}}_{B}, \omega\right)-B_{I}\left(\tilde{\mathbf{r}}_{B}, \omega\right)},
$$

that is directly $Z$, the digital filter definition (see Fig. 5.13). Moreover, with Eqs. 5.44 and 5.45, the impedance can be expressed in terms of the wave variables $P_{B, z}^{+}(\omega)$ and $P_{B, z}^{-}(\omega)$ 


$$
Z_{B}\left(\tilde{\mathbf{r}}_{B}, \omega\right)=Z_{p M} \frac{P_{B, z}^{-}(\omega)+P_{B, z}^{+}(\omega)}{P_{B, z}^{-}(\omega)-P_{B, z}^{+}(\omega)}
$$

Using the equivalence with the plane wave variables, Eqs. 4.61 and 4.62, this is expressed in terms of incident and reflected plane wave components $P_{i}\left(\tilde{\mathbf{r}}_{B}, \omega\right)$ and $P_{r}\left(\tilde{\mathbf{r}}_{B}, \omega\right)$ (see Sec. 2.4.1.3)

$$
Z_{B}\left(\tilde{\mathbf{r}}_{B}, \omega\right)=\frac{\rho_{0} c}{\cos \theta} \frac{P_{i}\left(\tilde{\mathbf{r}}_{B}, \omega\right)+P_{r}\left(\tilde{\mathbf{r}}_{B}, \omega\right)}{P_{i}\left(\tilde{\mathbf{r}}_{B}, \omega\right)-P_{r}\left(\tilde{\mathbf{r}}_{B}, \omega\right)} .
$$

Now, by means of the definition of the reflection factor in the plane wave, Eq. 2.65

$$
Z_{B}\left(\tilde{\mathbf{r}}_{B}, \omega\right)=\frac{\rho_{0} c}{\cos \theta} \frac{1+R\left(\tilde{\mathbf{r}}_{B}, \omega\right)}{1-R\left(\tilde{\mathbf{r}}_{B}, \omega\right)},
$$

which agrees with the definition of a locally reacting impedance, Eq. 2.70.

\subsubsection{Examples}

In order to demonstrate the validity of the proposed method in a cartesian mesh, some examples are presented. They correspond to the simulation of a frequency-dependent impedance surface, where the measured reflection factor is analyzed and compared with the theory at different angles. With the same set-up, some simulations are carried out with an interpolated cartesian mesh as well. Finally, some additional issues are addressed.

\section{Experimental set-up:}

The experiment presents the effect of one impedance surface when plane waves strike it at different angles of incidence. Let us consider a 2-D mesh with $2000 \times 1000$ cells in a cartesian topology, where the boundary condition is implemented in the middle of the largest side and with a length of 1000 cells. The rest of the boundaries have been treated with absorbing boundary conditions [Murphy and Mullen, 2002] to reduce the presence of reflections coming from the limits of the mesh. The size of the cells is determined using the Courant formula, Eq. 4.35, as an equality. The sampling frequency is $80 \mathrm{kHz}$, and from now on the frequency axes are presented normalized to the sampling frequency. Regarding to the source bandwidth and position, same guidelines than the ones used in Sec. 5.2.2.1 have been followed. 
In these simulations, the reflection factors are obtained and they are analyzed and compared with theoretical results as a function of the angle of incidence. These measured reflection factors are obtained as a difference between the spectrum of an incident plane wave source and the measured reflected signal. A total-field/scattering-field formulation has been used in order to minimize undesirable reflection effects [Umashankar and Taflove, 1982, Mur, 1981]. This formulation consists of carrying out two simulations with the same sources, but one with the proposed boundary condition and the other one without it. The scattering/reflecting field consists of the difference between both simulations. Also, a proper analysis window size according to the length of each one of the reflected impulse responses has been chosen in order to avoid the presence of undesirable reflections.

The frequency-dependent boundary condition is again modeled as a hard-backed layer of porous material (see Sec. 2.4.1.4).In accordance with the locally reacting impedance definition, Eq. 2.70, the theoretical reflection factor can be theoretically calculated for different angles. In this case, the angles of arrival under study $\theta$ are $0,15,30,45,60$ and 75 degrees. These theoretical reflection factors are indicated in Fig. 2.6.

The presented model is realized and compared not only with the expected reflection factors obtained analytically, but also with Huopaniemi's method.

The coefficients of the digital filter that represent the impedance have been modeled using an IIR digital filter of 60th order whose coefficients have been calculated according to Yule-Walker's algorithm using the analytical response of the absolute value impedance. Figure 2.5 also presents the complex components of the filter approach used to simulate the impedance. In the case of Huopaniemi's method, the reflection factor has been calculated from the locally reacting impedance definition and assuming a normal incidence $\left(\theta^{\prime}=0\right)$. The corresponding digital filter coefficients have been calculated using a 60th order IIR filter using Yule-Walker's algorithm as well, the response being represented in Fig. 5.14. From the discussion addressed in Sec. 2.4.1.4, the reflection factor used as a reference is the one obtained from the approached impedance spectrum and represented in the corresponding figure.

\section{Analysis of results:}

In accordance to the simulation configuration described above, a set 


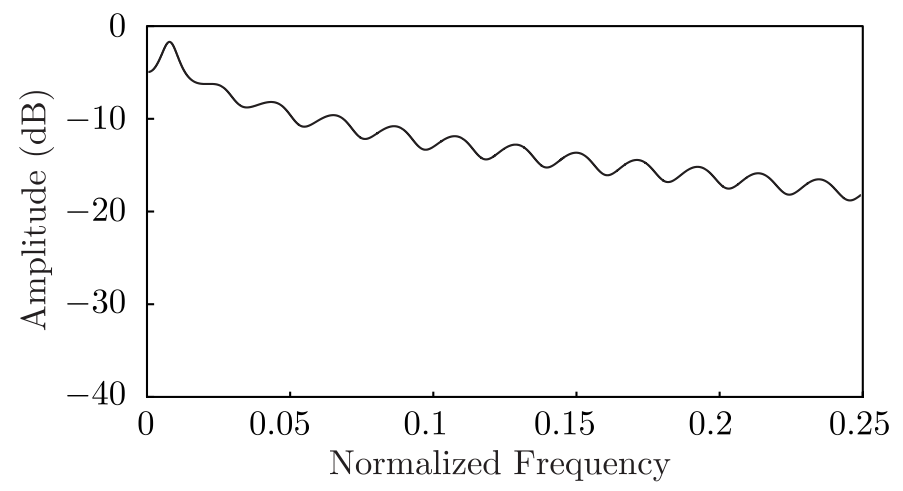

Figure 5.14. Module of the reflection factor used for Huopaniemi's method [Huopaniemi et al., 1997], calculated assuming an angle of arrival of 0 degrees.

of simulations have been performed. In Fig. 5.15, the reflection factors at the specified angles are presented. The reflection factor obtained according to the proposed method (solid line) is compared with the results obtained following Huopaniemi's method (dashed line) and with the expected theoretical results (dot-dashed line). Although Huopaniemi's method has some dependence on the angle of arrival, it is too far from a locally reacting impedance [Escolano and Jacobsen, 2007]. However, the results obtained from the proposed method show an evident agreement with the expected results. It is true that some errors appear for high frequencies, but they are expected since for frequencies near to 0.25 , the dispersion effects are presented for most angles of propagation, except for $\theta=45$ degrees (Fig. 5.15d)). For this particular scenario, dispersion is minimized, since this angle, in the particular case of a homogenously discretized mesh, is the same as the one that forms the diagonal direction with respect to the mesh coordinated system. At this angle, the inherent angular dispersion is minimized, independently of the oversampling used to reduce the frequency dispersion, and for that reason there is a high level of agreement between theory and results. For very low frequencies some discrepancies appear, but they are dependent on the window analysis size and the signal truncation.

This method shows great efficiency since it only requires the definition of just one filter, the impedance filter, even when the reflection factor is largely 

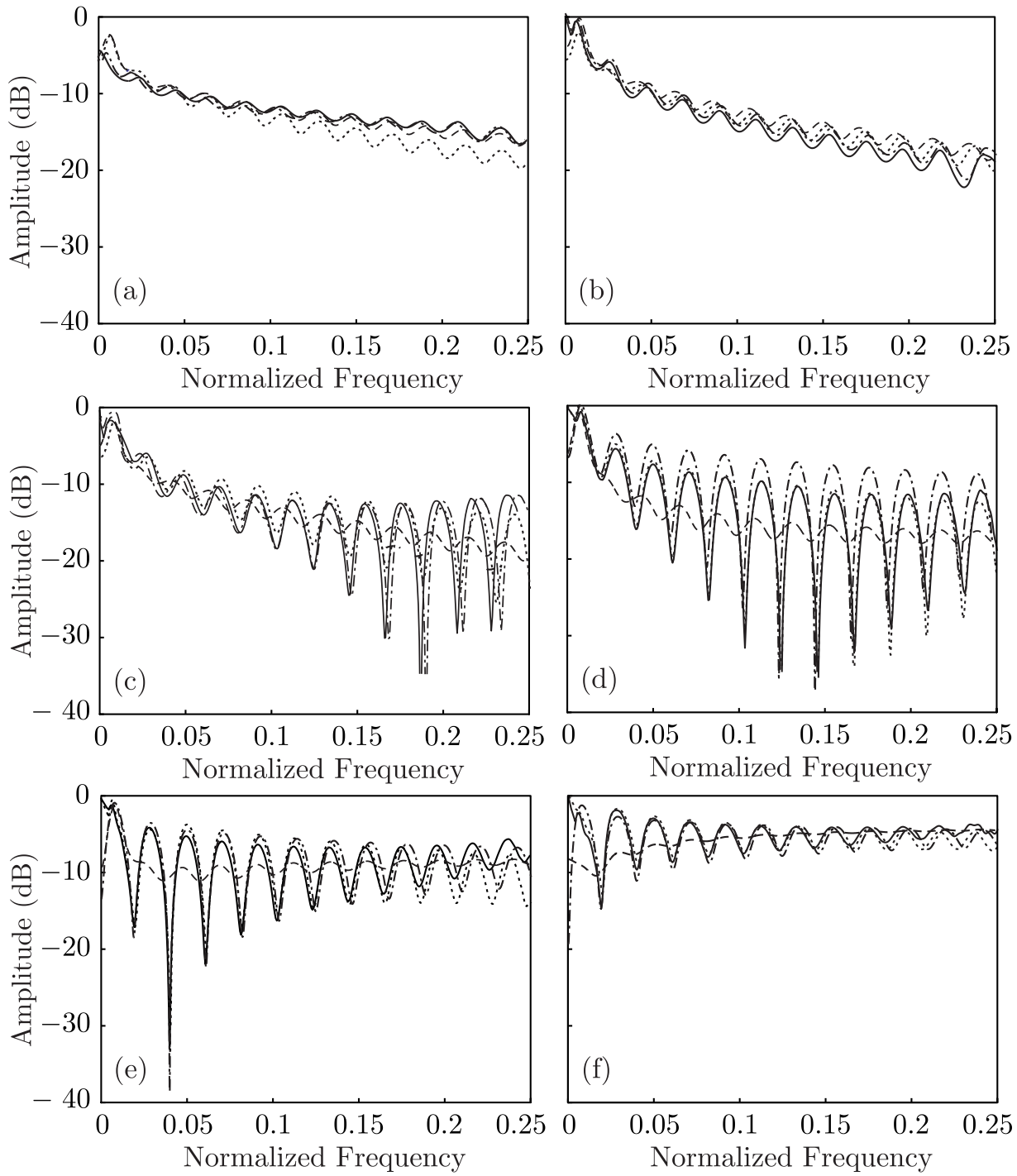

Figure 5.15. Reflection factors calculated with the proposed method for the cartesian mesh (solid line), for the interpolated cartesian mesh (dotted line) and with Huopaniemi's method [Huopaniemi et al., 1997] (dashed line), compared with the expected theoretical reflection factor (dot dashed line), for the angles: (a) 0 degrees, (b) 15 degrees, (c) 30 degrees, (d) 45 degrees, (e) 60 degrees, (f) 75 degrees. 
dependent on the angle of arrival and the spectrums are highly different between them. This is one of the advantages with respect to Huopaniemi's method, because even with prior knowledge of the angle of arrival, as shown in Sec. 5.2.3, it would be necessary to define a digital filter for each one of the possible angles. This would increase the computational cost and even in that case, the proper results would not be assured.

However, one of the main problems with this implementation is the efficiency of the W-DWM itself (see Sec. 4.3). Fortunately, the global efficiency of the system can be augmented using a hybrid mesh in the fashion of [Karjalainen, 2004] and [Murphy and Beeson, 2007]. As pointed out in Sec. 4.3, generally, a hybrid mesh consists of a K-DWM realization of the entire geometry and an interface with a W-DWM at the boundaries. The proposed method can be included in this hybrid mesh by simply coupling the model of Fig. 5.13 with a K-DWM via the KW-pipe proposed in [Erkut and Karjalainen, 2002b] (or with a more efficient version of this converter [Kelloniemi, 2006]) or the one in [Murphy and Beeson, 2007], more appropriate for the triangular DWM case.

At this point, some new simulations have been carried out under a hybrid mesh. However, since the KW-pipes have caused no errors [Karjalainen, 2004, Murphy and Beeson, 2007], the simulation carried out with this mesh has provided exactly the same result as using a W-DWM for the entire mesh. For that reason, no additional results are reported. Nevertheless, an interesting additional advantage of using KW-pipes is to couple an interpolated cartesian mesh [Savioja and Välimäki, 1997]. The interpolated mesh itself does not reduce the frequency dispersion; however it spreads the angular dispersion evenly and then, combining it with warping filtering, the dispersion is reduced considerably [Savioja and Välimäki, 2000]. The application of this mesh to room acoustic simulation has been frequently used [Kelloniemi et al., 2005, Kelloniemi, 2005a]. These results are also presented in Fig. 5.15 (dotted line) and it can be observed how this mesh, with any additional consideration for the boundary condition, is directly coupled to the proposed boundary condition. However, not just one dummy junction has to be taken into account in this case, but also the dummy junctions situated in the diagonal in respect to the scattering junction $p_{J}$, with their respective weights, according to [Savioja and Välimäki, 1997]. The results show how for high frequencies some differences exist in respect to the theory, similar to the cartesian mesh; however the spectrum 
shape is still highly similar. These results demonstrate how the use of the proposed method is also suitable for alternative mesh geometries, such as the interpolated cartesian mesh.

It should be mentioned that the idea of interfacing these paradigms has already been proposed by Karjalainen [Karjalainen, 2003], where in order to achieve a 1-D simulation, an interface between FDTD (or K-DWM), DWG and WDF was proposed. However, that work has demonstrated how this sort of interfacing provides additional advantages in a multidimensional simulation. In fact, this coupling system permits the direct definition of a locally reacting impedance to the FDTD method, taking into account the fact that it is also a pending problem for this method. This problem has so far only been approached in the room acoustic field by Kowalczyk and van Walstijn [Kowalczyk and van Walstijn, 2007], for non-frequency dependent boundary conditions or a simple spring-mass system [Kowalczyk and van Walstijn, 2008].

Another important feature to be considered is the linearity of the system. This is assured by the linearity of the digital filter and the interaction with the DWM. Let us assume two different plane waves with a different angle of arrival. If Huopaniemi's method is used with prior knowledge of the angle of arrival, it will be problematic because just one degree of freedom exists in the digital filter design and then, one of the plane waves would be filtered with an improper reflection factor filter. However, with the suggested method, this is not a problem because the impedance is constant with the angle of arrival and the system linearity permits the acquisition of a composition of reflected waves as if each one were obtained separately.

It should be noticed that this model makes sense in a multidimensional simulation, where the concept of the angle of arrival appears. In case of 1-D simulations, the Eq. 2.70 has $\theta=0$ and then, the same results are obtained with the previous approach as with the proposed model. However, in this particular case, the use of Huopaniemi's approach is more efficient than the presented one [Karjalainen, 2004], since the coupling with the digital filter representing the reflection factor does not need any kind of adaptation.

In Sec. 5.4, a real scenario is simulated by using the boundary condition algorithm developed during this section, and compared with real measurements, showing a great agreement between results. 


\subsection{Boundary conditions in the FDTD method}

Throughout this thesis, it has been commented several times how the FDTD approach (Botteldooren's approach, see Sec. 4.2) is not a really common technique for using in room acoustics, since the computational cost is drastically higher than the K-DWM approach. However, it remains popular for some acoustic applications such as aeroacoustics and outdoor sound propagation. Furthermore, during the last few years, some publications have appeared with some interesting contributions to the use of the FDTD in room acoustics, with special emphasis on the boundary treatment. However, few of them have been focused on the locally reacting impedance problem.

For that reason, it is worth trying to contribute to the FDTD method and how to define an efficient algorithm for locally reacting impedance modeling in a similar fashion to that presented in Sec. 5.2.4.

In this section, a short overview of the boundary condition implementation for the FDTD method is presented. During the next section, a method for the locally reacting impedance implementation is proposed [Escolano et al., 2008a].

\subsubsection{Previous approaches}

The impedance boundary condition in discrete-time method in acoustics, particularly for the FDTD method, has been reached in different ways for different acoustic topics, such as aeroacoustics [Özyörük and Long, 1997, Fung and Ju, 2004], outdoor sound propagation [Dong et al., 2000, Heutschi et al., 2005, Ostashev et al., 2005, Escolano and Pueo, 2007] and room acoustics [Botteldooren, 1995]. All those methods are based on modifications of Eqs. 4.14-4.17 in the boundary edges.

Since the total pressure, and also the particle velocity components appear during the algorithm, it is much more easier to work directly with the impedance. This method, considering a discrete time signal representation of the pressure and particle velocity, defines a relation between them as 


$$
P\left(e^{j \omega}\right)=\frac{\sum_{n^{\prime}=0}^{N-1} c_{n^{\prime}} e^{-j \omega n^{\prime}}}{1-\sum_{m^{\prime}=1}^{M-1} d_{m^{\prime}} e^{-j \omega m^{\prime}}} \mathbf{U}\left(e^{j \omega}\right) \cdot \hat{\mathbf{n}},
$$

where the frequency response of the impedance has been approximated by using rational expression. This approximation of the frequency response use to be seen as a IIR filter, which is usually carried out using Prony [Parks and Burrus, 1987], Yule-Walker [Friedlander and Porat, 1984] or Padé [Ostashev et al., 2007] algorithms.

The IIR filter structure in the frequency domain has a corresponding equation in the time domain [Proakis and Manolakis, 1998]. This difference equation,

$$
p(n)=\sum_{n^{\prime}=1}^{N} c_{n^{\prime}} \mathbf{u}\left(n-n^{\prime}\right) \cdot \hat{\mathbf{n}}-\sum_{m^{\prime}=1}^{M-1} d_{m^{\prime}} p\left(n-m^{\prime}\right),
$$

can easily be incorporated in the discrete time model, such as in [Özyörük and Long, 1997, Heutschi et al., 2005]. However, such a method requires storing both the sound pressure and the particle velocities at different time steps as coefficients, which leads to a significant increase of the required computer memory, since for each position $N$ particle velocity and $M-1$ pressure data points at the boundary cells must be stored from previous time steps. Furthermore, using such a function in a recursive convolution or using differential methods can be very time-consuming (see [Sullivan, 1992] for a detailed comparison of such methods).

Some proposed methods for boundary conditions have been focused on the room acoustic simulation: Olensen [Olesen, 1997] modified the FDTD formulation including a constant absorbing coefficient, but no analysis about the dependence with the angle of arrival has been presented. Suzuki [Suzuki et al., 2007] and Jeong [Jeong et al., 2007] implement frequency-dependent boundary conditions assuming a damper-spring-mass model of the wall impedance. Unfortunately, both not analyze the behaviour of their models in the locally reacting sense, only in the normal direction to the wall. In this sense, Kowalczyk and van Walstijn offer a similar method based on the same wall assumptions; however, the main contribution lies in the analysis 
of the method in terms of the locally reacting impedance model [Kowalczyk and van Walstijn, 2008].

These considerations lead to the conclusion that an implementation of an FDTD simulation incorporating frequency dependent boundary conditions will require significant computer resources and conditionally stable impedance boundary conditions [Fung and Ju, 2004]. One has to consider this modifies the Eqs. 4.14-4.17; it can be complicated to obtain a general expression for an indeterminate number of coefficients; and stability is not easy to ensure.

\subsubsection{Proposed method}

In this section, an algorithm for implementing a locally reacting impedance in an FDTD mesh based on the mixing modeling strategies (see Sec. 5.2.4) is presented. The scope of this model is to link the FDTD method with a rational representation of the impedance by means of a wave digital variable decomposition of both paradigms. Following a similar scheme than as the previous section, a WDF representation of the digital filter interacts with the wave propagation medium, which is represented in this case through the FDTD method. The separation and interaction in WDF terms ensures an unconditionally stable model when both parts (the FDTD model of the sound field and the digital filter representation of the impedance, see Sec. 5.2.4.2) are independently stable. The only source of instability, an implicit equation, is avoided using this variable conversion.

Then, following the same procedure than in Sec. 5.2.4, FDTD variable decomposition into WDF should be presented. It involves determining incoming and outgoing wave components at interface points of the FDTD simulations.

\subsubsection{Interaction between FDTD and wave variables}

The scope is to find a WDF decomposition at the interface point $\tilde{\mathbf{r}}_{B}$ in the incoming and outgoing components, where the sound pressure and the particle velocity components at $\tilde{\mathbf{r}}_{B}$ are unknown at the actual time step. These values depend on neighbouring values of the pressure and the particle velocity calculated using the FDTD algorithm. They are found by means of the FDTD discretized version of the Euler equation, Eq. 4.14-4.16.

As an example, a surface interface with a normal vector in the $x$ - 
direction component is considered. Let the medium be at $x<x_{B}$. The objective is to find $p\left(\tilde{\mathbf{r}}_{B}, n\right)$ and $u_{x}\left(\tilde{\mathbf{r}}_{B}, n\right)$ by means of $b_{x}\left(\tilde{\mathbf{r}}_{B}, n\right)$ and $a_{x}\left(\tilde{\mathbf{r}}_{B}, n\right)$, using Eqs. 5.29 and 5.30. The particle velocity component $u_{x}\left(\tilde{\mathbf{r}}_{B}, n\right)$ cannot be obtained directly from the FDTD scheme due to the staggered distribution of the variables. One possibility could be to determine this quantity as a temporal and spatial average of surrounding and previous points; cf. Eq. 4.14. However, although this approach would seem to be reasonable, a set of different approximations of $u_{x}(i, j, n)$ have been implemented and compared, and the results show that the case where the medium is at $x<x_{B}$, the approximating $u_{x}\left(\tilde{\mathbf{r}}_{B}, n\right)$ by $u_{x}\left(\tilde{\mathbf{r}}_{B}-\frac{1}{2} \Delta \tilde{\mathbf{x}}, n+\frac{1}{2}\right)$ is a better solution. In case the medium is at $x>x_{B}, u_{x}\left(\tilde{\mathbf{r}}_{B}, n\right) \simeq u_{x}\left(\tilde{\mathbf{r}}_{B}+\frac{1}{2} \Delta \tilde{\mathbf{x}}, n+\frac{1}{2}\right)$ should be used.

First $b_{x}\left(\tilde{\mathbf{r}}_{B}, n\right)$ is calculated. Note that the outgoing component depends on the pressure and the particle velocity at the interface at the present time step, but still remains unknown. In the $x$-direction, Eq. 4.14 is used. Solving for the pressure gives

$$
p\left(\tilde{\mathbf{r}}_{B}, n\right)=p\left(\tilde{\mathbf{r}}_{B}-\Delta \tilde{\mathbf{x}}, n\right)-\rho_{0} \frac{\Delta x}{\Delta t}\left(u_{x}\left(\tilde{\mathbf{r}}_{B}-\frac{1}{2} \Delta \tilde{\mathbf{x}}, n+\frac{1}{2}\right)-u_{x}\left(\tilde{\mathbf{r}}_{B}-\frac{1}{2} \Delta \tilde{\mathbf{x}}, n-\frac{1}{2}\right)\right)
$$

Inserting Eq. 5.64 into Eq. 5.29 gives, with $Z_{p}=\rho_{0} \Delta x / \Delta t$,

$$
b_{x}\left(\tilde{\mathbf{r}}_{B}, n\right)=p\left(\tilde{\mathbf{r}}_{B}-\Delta \tilde{\mathbf{x}}, n\right)+Z_{p} \cdot u_{x}\left(\tilde{\mathbf{r}}_{B-\frac{1}{2}} \Delta \tilde{\mathbf{x}}, n-\frac{1}{2}\right) .
$$

This particular value of $Z_{p}=\sqrt{2} \rho_{0} c$ with $c \Delta t=\Delta x / \sqrt{2}$ (cf. the Courant condition, Eq. 4.35) ensures that the outgoing component depends only on previous and known values of the pressure and the particle velocity and not on the boundary condition. The incoming component is obtained as the outgoing component in the other block. It is assumed that $a_{x}\left(\tilde{\mathbf{r}}_{B}, n\right)$ is known. Once the wave variables at interface points are known, Eqs. 5.31 and 5.32 are used to obtain both the pressure and the particle velocity at these points at instant $n$. The same process is necessary for calculating the $y$ and $z$-component.

In case the normal component of the wall $\hat{\mathbf{n}}$ has $n_{y}$ and $n_{z} \neq 0$, Eq. 5.31 may raise a double (or triple) representation of pressure. One can average all pressure values; however, it can produce dissipation problems ${ }^{5}$. A simple

\footnotetext{
${ }^{5} \mathrm{~A}$ scheme without dissipation can be used to integrate from given data at $t=0$ to a
} 
solution consist of just calculating the particle velocity components through Eq. 5.32 and then, obtaining the pressure by using Eq. 4.17.

The approach used for the spatial average of the particle velocity introduces some errors (undesirable reflections). One way of reducing these errors would be to use a more accurate approximation of the finite differences in the Euler equation. A third order finite difference approximation provides a good trade off between accuracy and computational cost [Strikwerda, 1989],

$$
\begin{aligned}
p\left(\tilde{\mathbf{r}}_{B}, n\right) & =\frac{4}{3} p\left(\tilde{\mathbf{r}}_{B}-\Delta \tilde{\mathbf{x}}, n\right)-\frac{1}{3} p\left(\tilde{\mathbf{r}}_{B}-2 \Delta \tilde{\mathbf{x}}, n\right) \\
& -\rho_{0} \frac{\Delta x}{\Delta t}\left(u_{x}\left(\tilde{\mathbf{r}}_{B}-\frac{1}{2} \Delta \tilde{\mathbf{x}}, n+\frac{1}{2}\right)-\frac{4}{3} u_{x}\left(\tilde{\mathbf{r}}_{B-\frac{1}{2}} \Delta \tilde{\mathbf{x}}, n-\frac{1}{2}\right)\right. \\
& \left.+\frac{1}{3} u_{x}\left(\tilde{\mathbf{r}}_{B}-\frac{1}{2} \Delta \tilde{\mathbf{x}}, n-\frac{3}{2}\right)\right) .
\end{aligned}
$$

Following the same procedure used in deriving Eq. 5.65, the outgoing component is achieved as

$$
\begin{aligned}
b_{x}\left(\tilde{\mathbf{r}}_{B}, n\right) & =\frac{4}{3} p\left(\tilde{\mathbf{r}}_{B}-\tilde{\mathbf{x}}, n\right)-\frac{1}{3} p\left(\tilde{\mathbf{r}}_{B}-2 \Delta \tilde{\mathbf{x}}, n\right) \\
& -Z_{p}\left(-\frac{4}{3} u_{x}\left(\tilde{\mathbf{r}}_{B}-\frac{1}{2} \Delta \tilde{\mathbf{x}}, n-\frac{1}{2}\right)+\frac{1}{3} u_{x}\left(\tilde{\mathbf{r}}_{B-\frac{1}{2}} \Delta \tilde{\mathbf{x}}, n-\frac{3}{2}\right)\right)
\end{aligned}
$$

with $Z_{p}=\sqrt{2} \rho_{0} c$. The two methods are compared in Sec. 5.3.2.3.

It should be emphasized that although third order approaches (and more generally, all approaches of odd order) are dispersive, this approach is only used at the boundary points and does not affect the propagation properties of the FDTD method used to simulate the sound field.

A demonstration of the performance of this approach is made through a 1-D FDTD simulation. This mesh, with a length $L$, is connected to a WDF resistor (see Sec. 4.7.2) with the same port variable as the one calculated in the FDTD mesh (see Fig. 5.16(a)). A gaussian source with a bandwidth of $0.25 f_{s}$ is propagated through the line and when it arrives at the

solution at $t=T$, and then, with time reversed, integrated back to $t=0$ to recover the data exactly (apart from roundoff errors). 
boundary linked to the WDF ports, what it is expected is a non reflection, since both parts are adapted in impedance. However, the signal/reflection ratio for both approaches, the second and third order ones, have been measured. For the second order finite differences the ratio is around $-27 \mathrm{~dB}$ (see Fig. 5.16(b)). However, using the third order finite differences approach this ratio has been reduced to about $-38 \mathrm{~dB}$ (see Fig. 5.16(c)). Unfortunately, this error seems to be frequency dependent, and it increases when the bandwidth also grows; however, it should be noted how the presented example has been limited to the conventional bandwidth used in room acoustics.

\subsubsection{Coupling model}

In a similar fashion to the one in Sec. 5.2.4, the proposed method consist in to plugging in a impedance filter realized through a WDF, see Sec. 5.2.4.2.

In the coupled model, each block sees the other block as a WDF port. In order to couple two WDFs and avoid instabilities, it is necessary to use a two-port parallel adaptor to adapt the impedance and avoid direct feedback loops (see Sec. 4.7.3). Once the interaction between the models has been specified, the frequency dependent FDTD model is shown in Fig. 5.17 for both approaches.

\subsubsection{Examples}

Some simulations have been carried out in order to examine the performance of the proposed method of realizing a frequency dependent impedance boundary condition. Examples in a 2-D mesh are presented. The impedance model selected for these experiments is again a hard-backed layer of porous material according to Sec. 2.4.1.4.

All the FDTD results presented here are based on a homogeneous discretization, with $\Delta x=\Delta y$. The sampling frequency is $80 \mathrm{kHz}$ and the spatial sampling has been determined from Eq. 4.35. In order to avoid reflections from the walls others than the one under test, absorbing boundary conditions have been implemented. In these cases, a Perfect Matched Layer [Yuan et al., 1997] boundary condition has been chosen. Following the same set-up as in the previous examples (see Sec. 5.2.2.1, 5.2.3.3 and 5.2.4.5), the source is a gaussian pulse covering up to at least the frequency $0.25 f_{s}$.

The FDTD model is again tested by determining the reflection factor and comparing with the analytical expression result based on a locally re- 


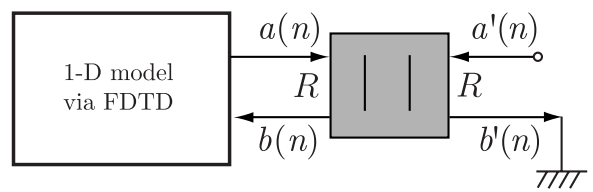

(a)
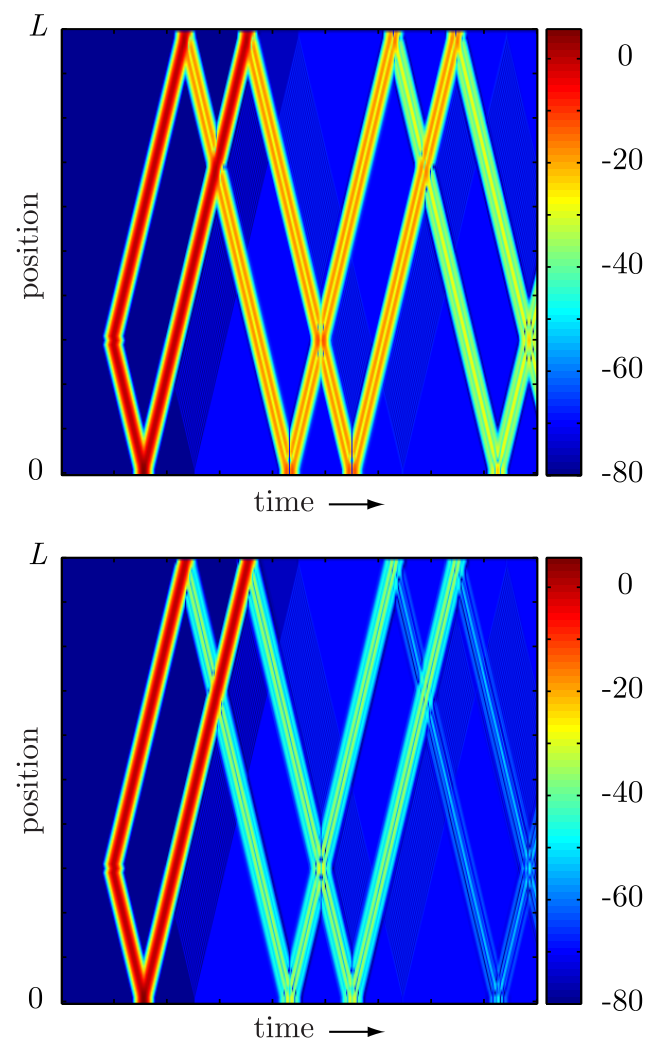

Figure 5.16. (a) 1-D setup for the determination of the error produce for the FDTD adaptation to wave variables. (b) Signal/reflection ratio for a second order approach. (c) Signal/reflection ratio for a third order approach. 

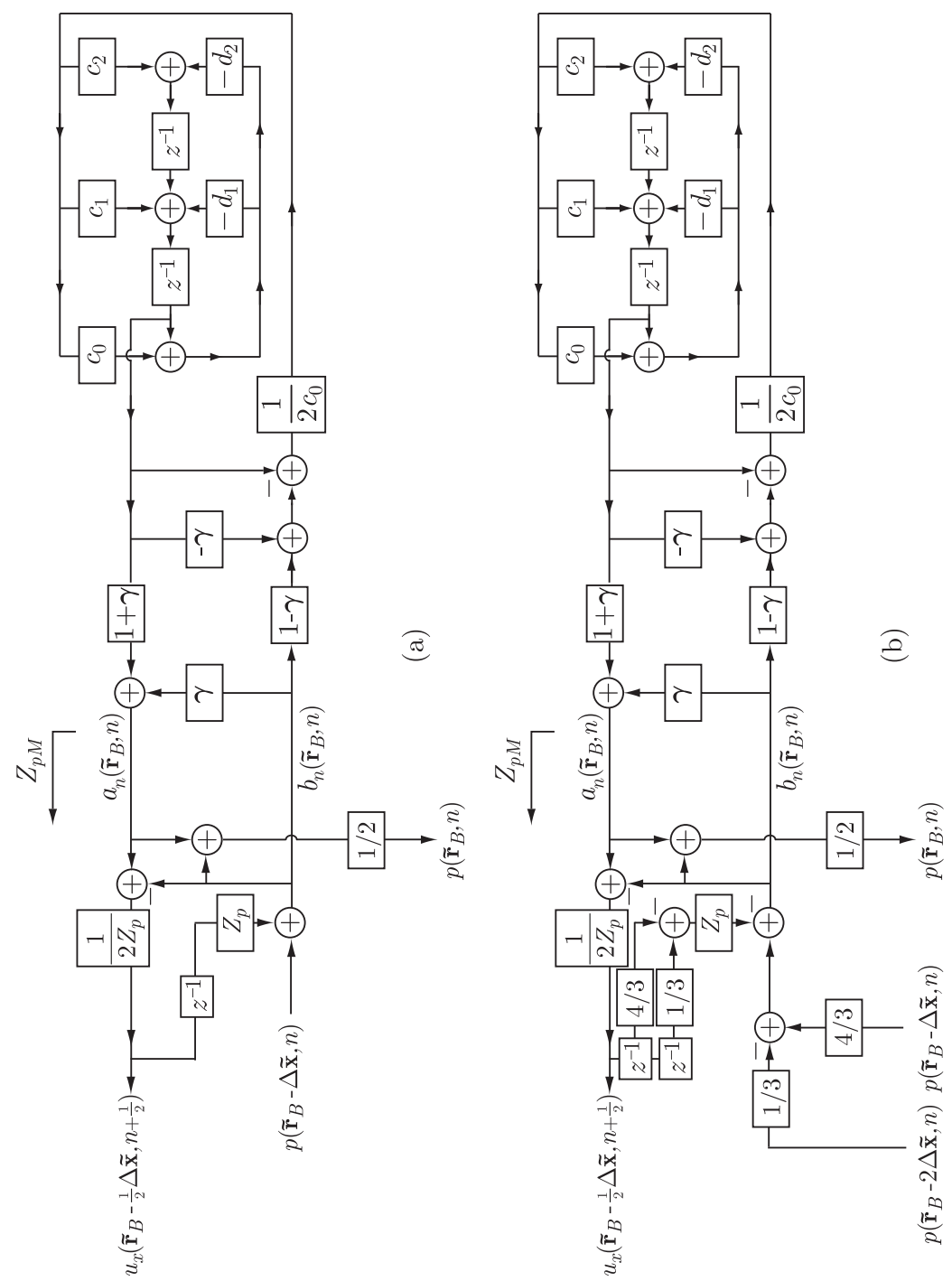

Figure 5.17. The Complete model of the proposed implementation by using (a) a second order and (b) a third order approach. The FDTD mesh and the impedance digital filter are implemented separately. A two-port parallel adaptor connects the two different models. 
acting impedance (see Fig. 2.6), following the same conditions for the source definition than in the Sec. 5.2.2.1.

It can be seen that in the second (dotted line) and third (solid line) order approach results shown in Fig. 5.18 there are similar to the analytical solution (dashed line) at all angles (0, 15, 30, 45, 60 and 75 degrees). However, there are also some deviations. The differences are partly due to the angle dependent artificial dispersion of the FDTD method [Taflove, 1998] (even though the frequencies have been selected for minimal dispersion effects); this effect can be observed in Fig. 5.18(d), which corresponds to an angle of incidence of 45 degrees, and the error is very small (in the FDTD mesh used here, this is the angle with no dispersion). However, the most significant source of error is due to the WDF approach in the FDTD mesh as described in Sect. 5.3.2.1: undesirable reflections can appear depending on the incident waveform. Because of such reflections some differences between theoretical and observed reflection factor levels occur, especially with the second order approach. This second order deviates considerably from the level of frequency response in the theoretical one, at least for angles between 0 and 45 degrees. It is also observed how these errors decrease when $\theta$ is increased. However, there exists a higher agreement when the third order approach to the wave variables is used. The maximum difference can be seen to be about $2 \mathrm{~dB}$ in most of the results until the frequency $0.125 f_{s}$; from this frequency in advance some more important deviations occur with angles near to the normal directions, although, they seem to be no longer than $6 \mathrm{~dB}$ in the frequency $0.25 f_{s}$. On the other hand, the high agreement with the expected reflection factor in the rest of angles of arrival in the entire frequency range, confirming the validity of the method can be observed. Then, it is worth emphasizing that the results follow a locally reacting model without any artificial modification of the boundary condition filter.

So far the model has been tested in an idealized situation where one single plane wave is incident on an impedance wall. However, in realistic situations several plane waves may well be incident simultaneously. In Fig. 5.19 is shown a case where two simultaneous plane waves strike the impedance surface at angles $\alpha_{1}=30$ degrees and $\alpha_{2}=60$ degrees.

In order to validate the linearity of the proposed method, three simulations are carried out, two in which the two plane waves are launched separately, and a third one where the two plane waves are incident at the 

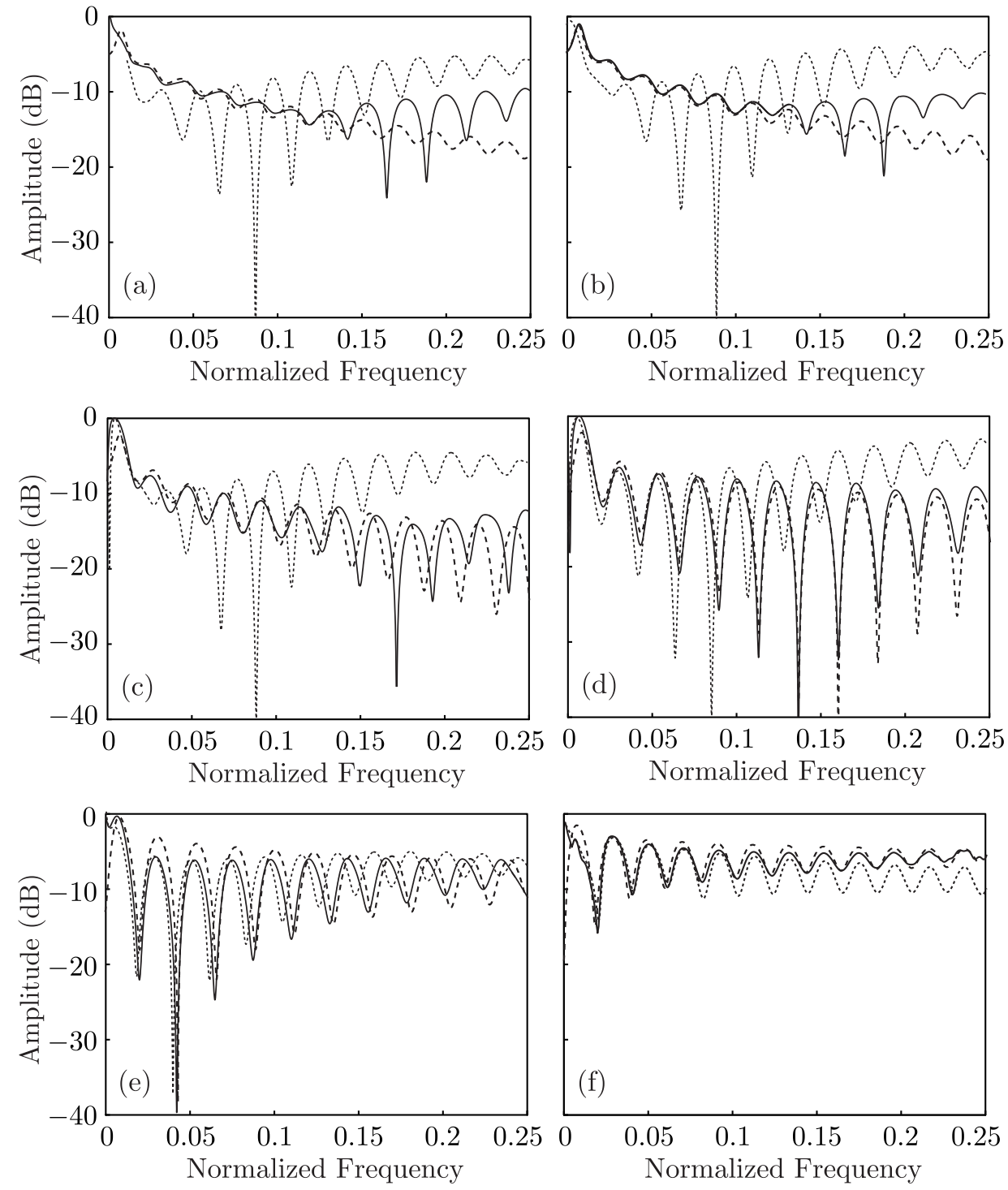

Figure 5.18. Reflection factor calculated with a 2-D FDTD mesh using a second order(dotted line) and a third order (continuous line) approach, compared with the theoretical reflection factor (dashed line), for the angles: a) 0 degrees, b) 15 degrees, c) 30 degrees, d) 45 degrees, e) 60 degrees, f) 75 degrees. 


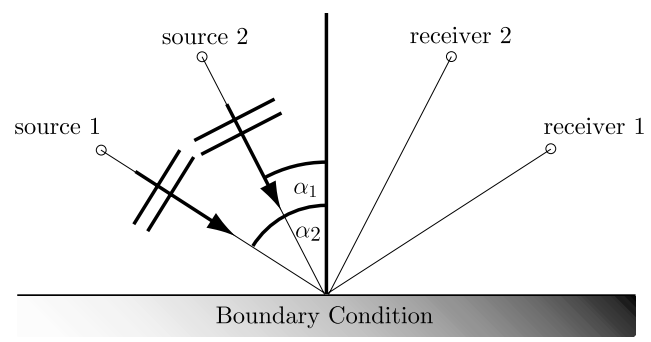

Figure 5.19. Set-up used for analyze the linearity of the proposed method.

same time. These analysis are done in the time-domain. Two different cases are presented, a first one where sources have a broadband $0.125 f_{s}$ and the other one with $0.25 f_{s}$. These bandwidth have been selected since the results shown higher agreement in frequencies up to $0.125 f_{s}$, whereas from this frequency up to $0.25 f_{s}$, some noticeable differences appear.

These impulse responses waveforms are more sensitive to the inherent dispersion. For this reason the impulse responses represented in Fig. 5.20 and Fig. 5.21 have been represented following a minimum-phase consideration (which means that they have the same magnitude response as the original systems; however, the energy is concentrated near the start of the impulse responses and they have minimum group delay) and only delays relative to the time difference of arrival have been taken into account; possible effects of the FDTD mesh angular dispersion are not considered in this time domain analysis.

Firstly, the results are analyzed for the bandwidth of $0.125 f_{s}$. In Fig. 5.20(a), the results at receiver 1 appear (see Fig. 5.19). In all the presented impulse responses the results from the simulations (continuous lines) are compared with the expected theoretical results (dotted lines). Fig 5.20(a.1) shows the impulse response when only the plane wave at $\alpha_{1}=30$ degrees is emitted, and Fig 5.20(a.2) shows the impulse response with the plane wave at $\alpha_{2}=60$ degrees. Fig. 5.20(a.3) shows the impulse response that results when both plane waves strike the boundary at the same time. The linearity is demonstrated when the result is compared with Fig. 5.20(a.4), that is, the result obtained if both independent plane waves are added (Fig. 5.20(a.1) and Fig. 5.20(a.2)). Comparing Figs. 5.20(a.3) and 5.20(a.4) shows that there is no difference between them. As well as 


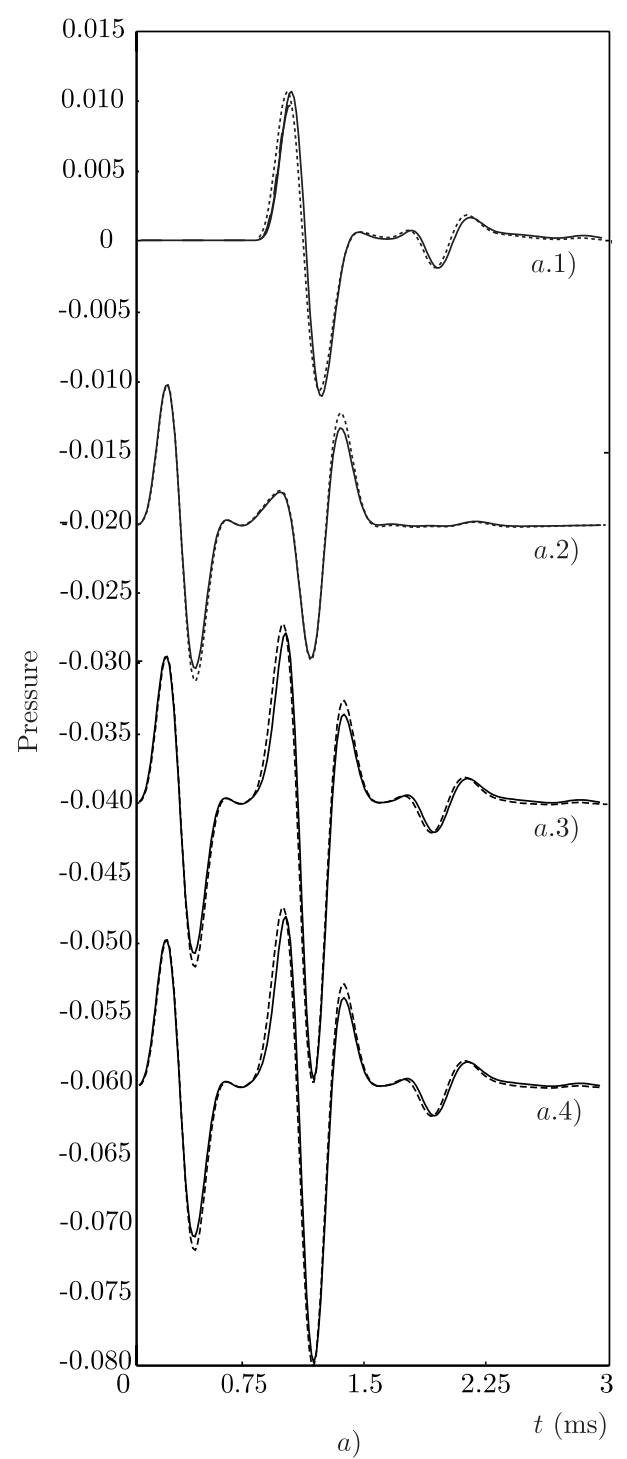

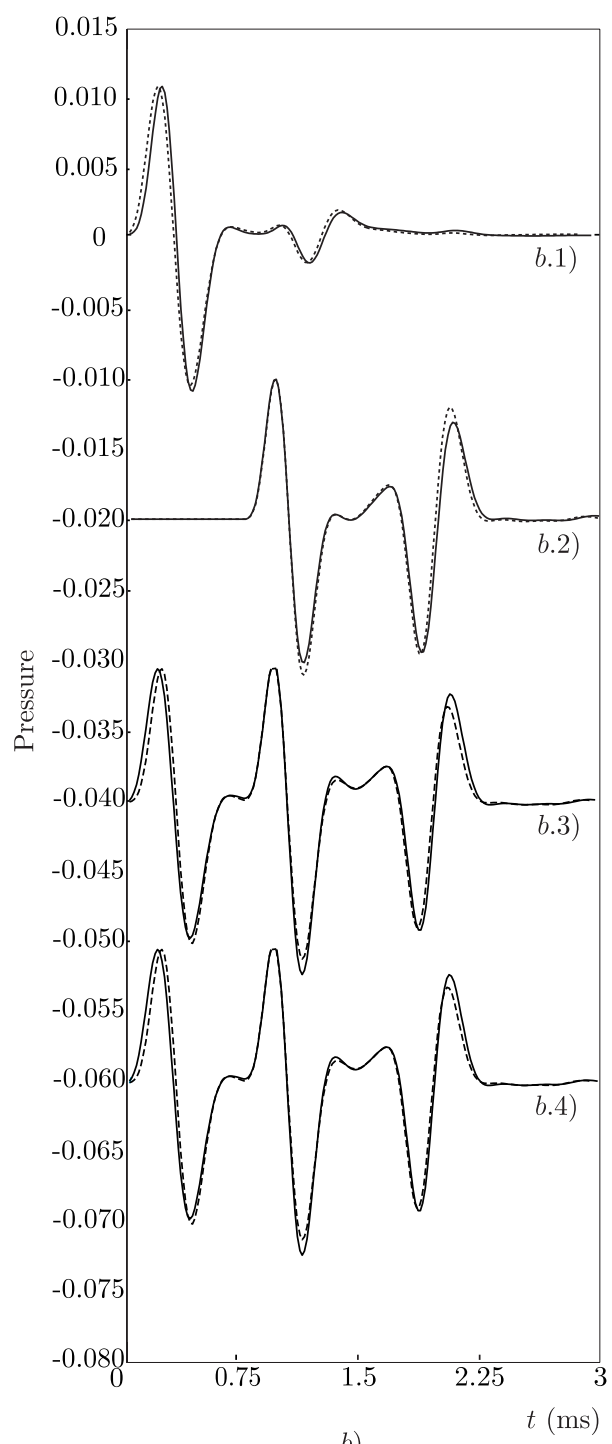

b)

Figure 5.20. 0.125 Impulse responses obtained in the simulation (solid line) versus theoretical results (dotted line) by using a gaussian source with broadband $0.125 f_{s}$ : a) at the receiver 1: a.1) with plane wave at $\alpha_{1}=30$ degrees, a.2) with plane wave at $\alpha_{2}=60$ degrees, a.3) both plane waves at the same time, a.4) adding both separated plane waves; b) at receiver 2: b.1) with plane wave at $\alpha_{2}=60$ degrees, b.2) with plane wave at $\alpha_{1}=30$ degrees, b.3) both plane waves at the same time, b.4) adding both separated plane waves. Each impulse response is shifted $-20 \mathrm{mPa}$. 

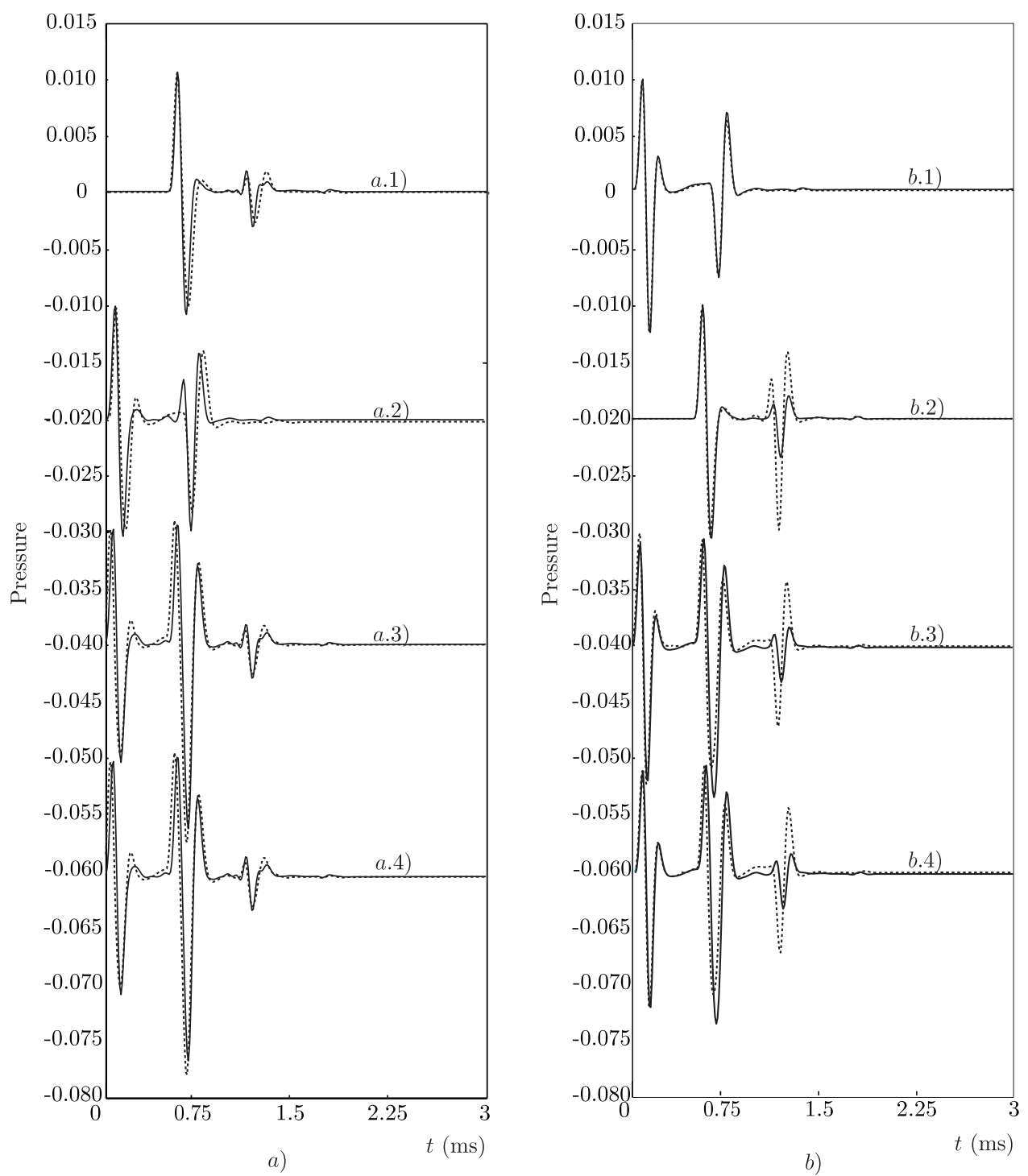

Figure 5.21. 0.25 Impulse responses obtained in the simulation (solid line) versus theoretical results (dotted line)by using a gaussian source with broadband $0.25 f_{s}$ : a) at the receiver 1 : a.1) with plane wave at $\alpha_{1}=30$ degrees, a.2) with plane wave at $\alpha_{2}=60$ degrees, a.3) both plane waves at the same time, a.4) adding both separated plane waves; b) at receiver 2: b.1) with plane wave at $\alpha_{2}=60$ degrees, b.2) with plane wave at $\alpha_{1}=30$ degrees, b.3) both plane waves at the same time, b.4) adding both separated plane waves. Each impulse response is shifted $-20 \mathrm{mPa}$. 
the agreement between the linear combination of the simulated impulse responses, those results agree with the expected ones. The same agreements are found in the receiver 2 (Fig. 5.20(b)).

It can be seen that there is no significant difference between the results and the expected theoretical results. The comparison between the results when the two plane waves appear at the same time and the result of adding the corresponding separate results demonstrates again the linear behaviour: there is no difference between the results.

However, from the results obtained for a source with bandwidth $0.25 f_{s}$, some disagreement are expected to be found, according to the spectrum responses (see Fig. 5.18). The same procedure followed as with the previous source is followed. In this case, the linearity is also demonstrated, as it is expected. However, some of those awaited differences in the impulses responses can be observed, particularly the impulse response coming from the source situated at angle $\theta=30$, which agrees with its corresponding spectrum.

\subsection{Experimental evaluation of boundary conditions in a DWM}

In this section, the scope is to validate through experiments and measurements the accuracy of those boundary conditions in a DWM, not only through comparison with analytical models [Kelloniemi, 2006], but also through comparison with measurements obtained in real scenarios, in a similar fashion to [Tsingos et al., 2002]. These comparisons do not appear in the technical literature for the DWM topic, focused on the boundary conditions, and they will result in valuable information to determine the accuracy of the model and particularly, for the frequency dependent boundary conditions. They may result in a key information to determine the accuracy of the model and particularly, for the frequency-dependent boundary conditions [Escolano et al., 2008b]. In this section, the validated boundary conditions are proposed in Sec. 5.2.4. 


\subsubsection{Measurement set-up}

The real scenario is built with two sandwich panels over a grass floor. The materials of the panels are made with a core of expanded polystyrene and a skin layer (at both sides) made with pressed aluminium. Both panels have dimension of $150 \times 120 \times 5 \mathrm{~cm}$ and they have been arranged in Fig. 5.23. A scheme of the distribution with their corresponding distances can also be found in Fig. 5.22.

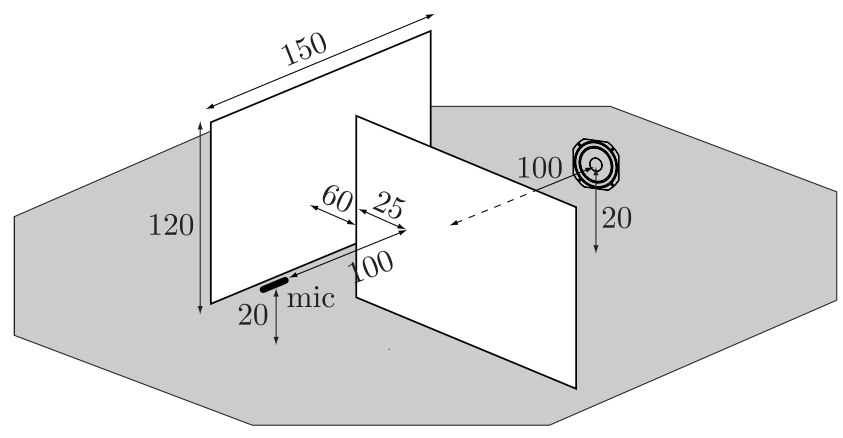

Figure 5.22. Scheme and measures of the scenario. All distances are given in centimeters.

A sound source (a dodecahedral loudspeaker) is situated at $1 \mathrm{~m}$ far from panel 1. The source impulse response consist on a Maximum Length Sequence [Rife and Vanderkooy, 1989] with order 16 and a sampling frequency of $48 \mathrm{kHz}$. The microphones are also situated $1 \mathrm{~m}$ away from panel 1 , but at the opposite side than to the loudspeaker. Under these conditions, a measurement in the indicated position is carried out and the results will be presented later.

The next step after this measurement is to characterize the materials introduced in the scenario. This means to measure the impedance function of the materials presented in the scenario. For this purpose, the method proposed by Takahashi et al. is used, where two close microphones are arranged to determine the reflection factor and impedance under a locally reacting surface assumption [Takahashi et al., 2005]. The first microphone is situated at $15 \mathrm{~mm}$ from the panel and the second one is situated at 17 $\mathrm{mm}$ from the panel as well. Measuring with this setup at different angles (0, 15, 3045 and 60 degrees), the impedance is obtained through an average of these results (see [Takahashi et al., 2005] for more details). Once the 


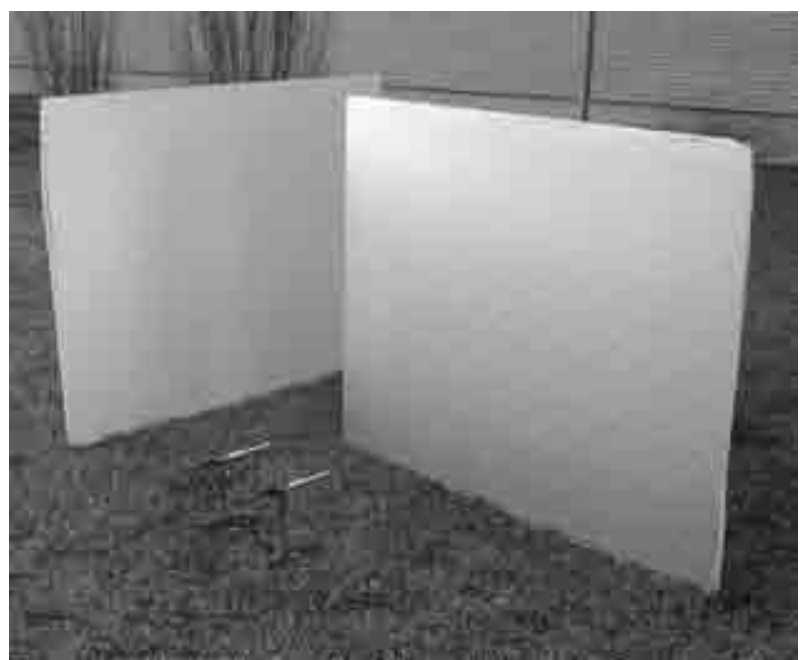

a)

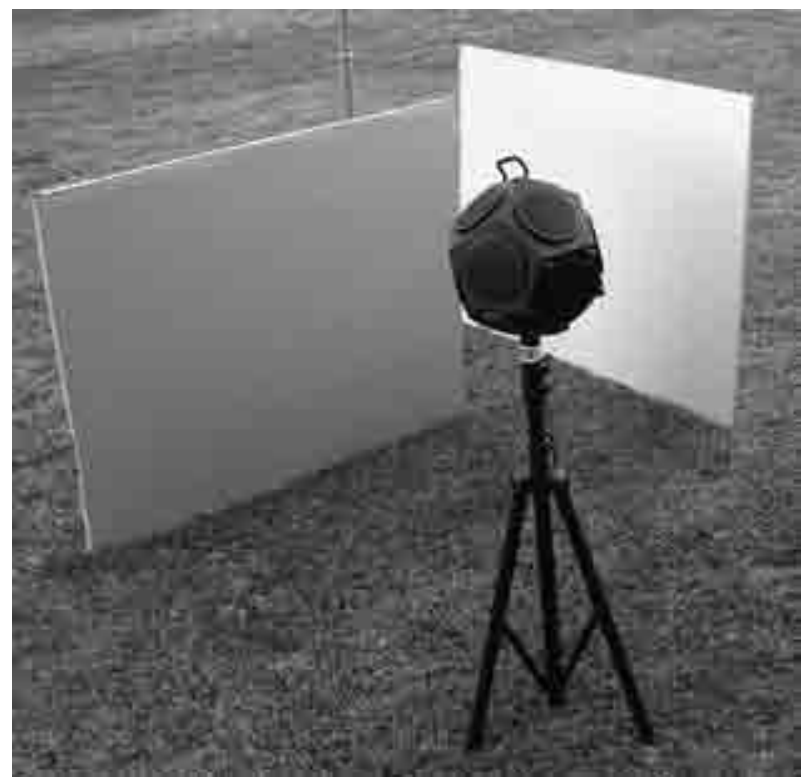

b)

Figure 5.23. Different pictures of the real scenario used during the measurements, including the position of the loudspeaker and the microphone. 
spectrum of the impedance is achieved, through the Yule-Walker algorithm, the coefficients of an IIR digital with order 30 are updated [Proakis and Manolakis, 1998]. The amplitude (solid line) and phase (dashed line) of the achieved filter are represented at Fig. 5.24.

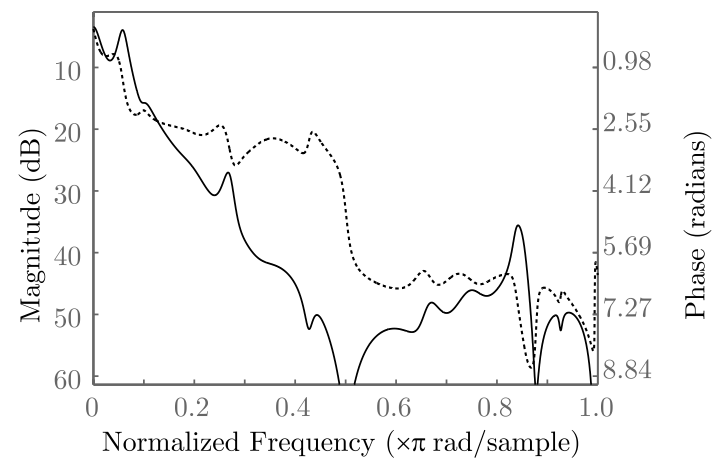

Figure 5.24. Amplitude and phase of the corresponding filter representation of the panel impedance. The solid line represents the amplitude and the dashed line, the phase.

Regarding the grass floor, the impedance filter has not been defined through only measurements, but also, a well-known model of the grass floor impedance has been used instead [Attenborough, 1992, Taherzadeh and Li, 1997], whose results have been validated through numerous examples in the technical literature. A fifth order is enough to achieve the corresponding IIR filter through the Yule-Walker algorithm.

\subsubsection{Simulation set-up}

Now, a DWM simulation following the same physical structure as in the experiment (see Sec. 5.4.1) is going to be carried out (see Fig. 5.22). Two simplifications have been performed: first, no transmission factors in the panels have been taken into account; and the second one is not to consider depth in the panels. Besides that, a decimation of the sampling frequency has been carried out, since this frequency determines the minimum size of the discretization step and the higher one is the sampling frequency, the more computational cost and memory require the simulation. Then, measurements have been downsampled to $40 \mathrm{kHz}$ in order to match the simulation. 
A simulated scenario with $200 \times 200 \times 200$ cells has been implemented with a regular discretization in a cartesian mesh. The size of the discretization cell corresponds with $1.48 \mathrm{~cm}^{3}$ according to the Courant condition (Eq. 4.35). Some absorbing boundary conditions are used at the boundaries. In this case, the Higdon condition [Higdon, 1986] gives a very good results and since it needs only pressure variables, it results in a proper candidate for these simulations. Through the algorithm of Sec. 5.2.4, the boundary conditions of the panels and the grass floor have been included in the simulation through their corresponding filters. The main algorithm is supported by a K-DWM, but a transformation to W-DWM is used at boundaries [Karjalainen, 2004], and the corresponding impedance filter is connected to their precise scattering junctions.

Since the inherent dispersion of the mesh modifies the spectrum of the results, it is necessary to solve this handicap. A common solution is to present the results up to a quarter of the sampling frequency [Duyne and Smith, 1993]. From now on, the results are presented in a normalized frequency scale in respect to the sampling frequency, where the maximum frequency corresponds with 0.25 .

\subsubsection{Discussion of the results}

In Fig. 5.25, the results through simulation and measurement are obtained. These results are expressed by means of the spectrum of the impulse response recorded in the microphone position. Regarding the measurements, some precautions have been taken into account due to some walls and objects, even although situated sufficiently far away from the measurement setup, were presented during the measurement process. However, by considering the distance to those significant objects, it has been possible to determine the size of a proper window analysis which mostly contains the signal influenced by the panel and the grass floor.

It can be observed how the results show a high level of agreement. Most important differences seems appear at low frequencies. It has to be observed that these errors may appear due to the measurement process of the impedance, where the very low frequencies could not be correctly measured because of the finite length between both microphones [Jacobsen, 2002]. Beside the fact some differences exist, to conclude about the validity of the boundary condition is reasonable to accept. 


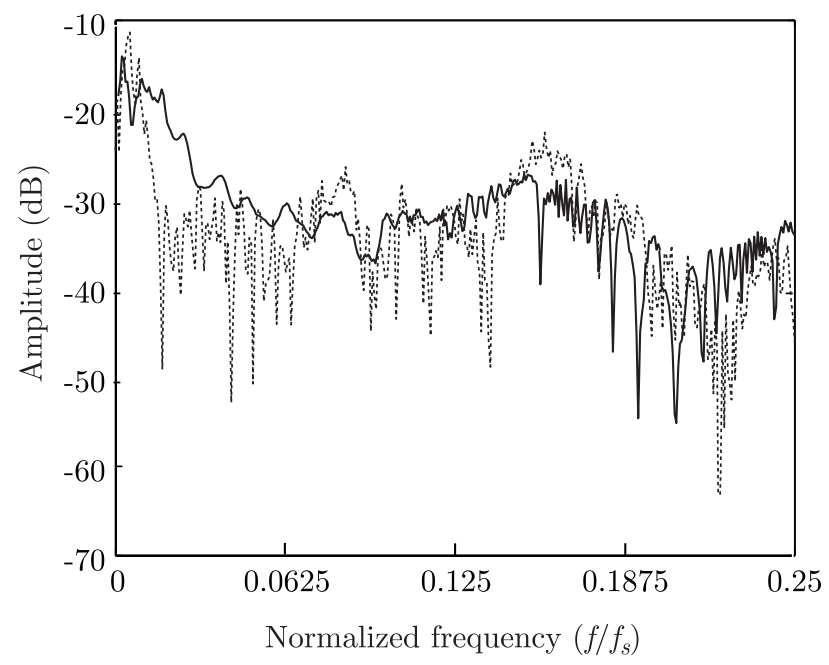

Figure 5.25. Spectrum of the impulse responses obtained through simulations (solid line) and measurements (dashed line).

Regarding the DWM dispersion, some expected deviation of the frequencies of the simulation compared with the measurements is observed. These deviations are visible since a tendency exists to move the high frequencies to low frequencies. However, since the simulation has been bandlimited to the normalized frequency 0.25 , the deviation is acceptable and confirms the validity of the simulation.

\subsection{Discussion}

This chapter deals with the boundary condition definition in discrete-time methods, focused on specular reflections with a frequency-dependence. Contributions to this topic are introduced to both DWM and FDTD methods, composing the two sections into which this chapter has been divided.

The use of the DWM, and particularly the K-DWM, has been considered as advantageous from the boundary condition point of view. The common boundary conditions in the DWM method are defined by using reflection factor filters (Huopaniemi's method); however, in multidimensional 
simulations this is problematic since the reflection factor is dependent on the plane wave angle of arrival and those filters have no information about it. The process is simplified by assuming a normal incidence for the filter realization.

An analysis of the effect of choosing the reflection factor as a boundary condition is introduced and how it works according to the plane wave angle of incidence. It has been observed how the measured reflection factor has some dependence on this angle, however, results shown how far they are from the locally reacting impedance.

According to this, two different solutions are offered: the first one consist on detecting the angle of arrival and perform a time-varying reflection factor digital filter according to this detected angle; and a second option consist into plug in an impedance filter which is independent of the plane wave direction and it matches with the locally reacting impedance definition.

The first method could be seen as an evident solution of Huopaniemi's method and results shown a good agreement with the expected from theory, but its computational cost makes it very difficult to implement in a complete room acoustic simulation program. On the other hand, the use of an impedance as a boundary condition produces a highly accurate solution with a reduced computational cost. This second option consists on the use of Wave Digital Filters as a common interface between the impedance filter and the DWM method. The advantage of this method is that the stability is assured if both elements are separately stable, because of delay-free loops (implicit equations) are avoided. The proposed method is able to model a locally reacting impedance without introducing any substantial increase of the computational cost.

Following this model, it is also possible to define a frequency dependent absorbing boundary conditions in a FDTD mesh. The FDTD mesh and the boundary conditions (also an impedance model defined by means of a digital filter) are implemented separately and joined using an interface based on Wave Digital Filters. Another important feature is that the definition of the digital filter can be designed with highly efficient structures, reducing considerably the computational cost of the whole algorithm.

Finally, in order to check the accuracy of these methods in a real scenario, some experimental measurements have carried out and compared 
with the same simulated scenario. This comparison shows an elevated degree of similarities between both results, outperforming the state-of-the-art methodologies. 


\section{Directive Sources in Discrete-Time Methods}

The DiscRete-Time methods ARE ONLY ABLE TO DEAL WITH POINT SOURCES, as has been shown in Chap. 4. Also plane waves could be defined as a "continuous" array of point sources with suitable phase. In the particular case of the FDTD method, it is also possible to introduce dipole sources just by defining the corresponding source function through a particle velocity component source (see Sec. 4.2). However, in practical and more general problems, sources are too far to be represented as monopole or dipole sources and more complex source modeling is required.

Sound source modeling aims to achieve realistic sound inside a virtual environment with characteristics resembling those of a real source. One of these characteristics is the directivity [Savioja et al., 1999], whose effects over the sound perception of the virtual room becomes highly significant [Dalenbäck et al., 1993, Otondo and Rindel, 2004].

Furthermore, in order to obtain information regarding the directivity of real sources as a function of the angles in a 3D space and also as a function of the frequency in the bandwidth of interest, complex facilities are required, e.g. anechoic chambers and measuring set-up [Flanagan, 1960, Huopaniemi et al., 1999]. However, directivity diagrams of sources for discrete frequencies are more common and they are available in technical literature [Fletcher and Rossing, 1991, Ochmann, 1995]. Nowadays, available public data-bases 
on this topic are not widespread; however, it must be highlighted a completed data-base of musical instruments directivities has been announced on the middle of next year 2009 [Lokki and Savioja, 2008].

\subsection{Introduction}

Sound source modeling has been an important topic which has been addressed in other room acoustic simulation methods, such as image-source methods [Huopaniemi et al., 1994], ray-tracing [Vigeant et al., 2004] or FEM [Ochmann, 1999]. Although some time-domain formulations exist [Kropp and Svensson, 1995], complex directive sources have only been proposed, very recently, in a particular discrete-time method - the W-DWM method [Hacıhabiboğlu et al., 2007]; however, this is not a generalized method that can be used in the rest of the methods, since it uses the proper weights of the outgoing components of the scattering junction where the source is situated, and it only allows frequency-independent implementations, being specially suitable for dipole and quadrupole representation. A method able to solve more complex problems (frequency dependent directivity) and able to deal with more efficient discrete-time methods, such as K-DWM, should be proposed [Svensson, 2004]. Unfortunately, the current state-of-the-art is not more extensive that this short paragraph, with the exception of, at this moment, an unpublished forthcoming paper [Hacihabiboğlu et al., 2008b].

In this thesis, and particularly in the current chapter, some methods have been proposed of incorporating directive sources for discrete-time methods, based on the information obtained in the directivity diagrams for discrete frequencies [Escolano et al., 2007] and also extended for broadband sources with variable directivity as a function of the frequency.

This chapter is summarized as follows: Sec. 6.2 introduces a method for synthesizing sinusoidal directive sources, and several examples will support it, including an analysis of the dispersion effect occurring when the method is applied to different discrete-time methods (see Sec. 6.2.2). Section 6.3, deals with the extension of the previous method for sources with a determined bandwidth and examples will be presented with constant and variable directivity. Finally, this chapter is summarized. 


\subsection{Sinusoidal directive sources}

As mentioned in the introduction, in the current section a method is proposed which tries to simulate the behavior of a directive source when it is used in discrete-time simulations. To accomplish this task it uses an array of basic sources (monopoles) placed around the position of the directive source to be synthesized (see Fig. 6.1(a)). The method is based on the combination of an array of monopoles with different amplitudes and phases in order to reproduce a desired sound field at given points [Wang and $\mathrm{Wu}$, 1997]. If such points are selected for spherical distribution in a far field, the resulting pressure pattern is directly the directivity diagram of the source.

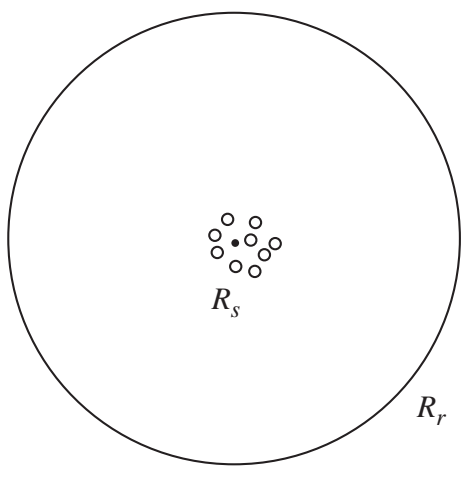

(a)

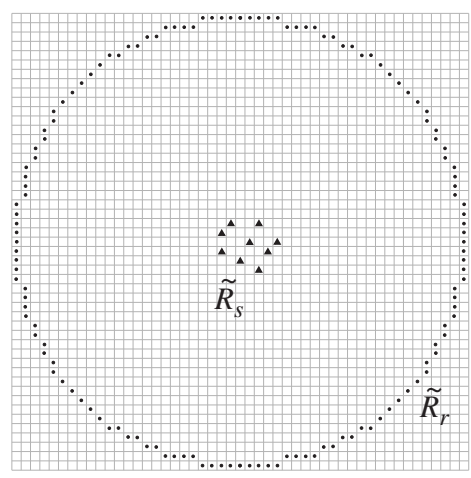

(b)

Figure 6.1. (a) Source-receiver points distribution. (b) Space sampled distribution of source-receiver points

Therefore, the objective is to obtain the proper combination of signals that excite the source distribution in order to obtain the desired angledependent behavior. However, the space sampling process forces the source and receiver points to be located at discrete positions (see Fig. 6.1(b)), but it will be shown that this will not cause any problems. Next, the algorithm is mathematically formulated in terms of the wave propagation.

Let us consider a sinusoidal 3D time-varying sound field (Eq. 2.1)

$$
p(\mathbf{r}, t)=P\left(\mathbf{r}, \omega_{\mathrm{o}}\right) e^{-j \omega_{\mathrm{o}} t},
$$

where $P\left(\mathbf{r}, \omega_{\mathrm{o}}\right)=\left\|P\left(\mathbf{r}, \omega_{\mathrm{o}}\right)\right\| e^{j \angle P\left(\mathbf{r}, \omega_{\mathrm{o}}\right)}$ is defined as the complex pressure 
amplitude of the sound field in a position $\mathbf{r}$ for the angular frequency $\omega_{\mathrm{o}}$ (see Sec. 2.2).

Also, let us define source matrix position $\underline{\mathbf{r}}_{s}=\left[\mathbf{r}_{s_{1}}, \ldots, \mathbf{r}_{s_{n}}, \ldots, \mathbf{r}_{s_{N}}\right]^{T}$ and receiver matrix position $\underline{\mathbf{r}}_{r}=\left[\mathbf{r}_{r_{1}}, \ldots, \mathbf{r}_{r_{m}}, \ldots, \mathbf{r}_{r_{M}}\right]^{T}$, where upperindex $T$ indicates transposed vector. Note that for this purpose, $\underline{\mathbf{r}}_{r}$ must correspond to a circular distribution as shown in Fig. 6.1(a). The pressure at a point $\mathbf{r}_{r_{m}}$ is calculated as a weighted sum of the pressure sources as

$$
P\left(\mathbf{r}_{r_{m}}, \omega_{\mathrm{o}}\right) e^{-j \omega_{\mathrm{o}} t}=\frac{1}{4 \pi} \sum_{n=1}^{N} P\left(\mathbf{r}_{s_{n}}, \omega_{\mathrm{o}}\right) e^{-j \omega_{\mathrm{o}} t} \frac{e^{j \frac{\omega_{\mathrm{o}}}{c}\left\|\mathbf{r}_{s_{n}}-\mathbf{r}_{r_{m}}\right\|}}{\left\|\mathbf{r}_{s_{n}}-\mathbf{r}_{r_{m}}\right\|} .
$$

From now on, let us define for simplicity $\left\|\mathbf{r}_{s_{n}}-\mathbf{r}_{r_{m}}\right\|=\left\|\mathbf{r}_{s_{n}, r_{m}}\right\|$.

The weights that relate the pressure distribution of the sound field to the sound sources are related to the three-dimensional Green's functions [Pierce, 1994]. In Eq. 6.2, Green's function for point sources in an unbounded sound field is employed. Assuming a far field approximation $\left(\frac{\omega_{\mathrm{o}}}{c}\left\|\mathbf{r}_{s_{n}, r_{m}}\right\| \gg 1\right)$, or equivalently a plane wave approach, Green's function can be simplified. This simplification is made assuming that in a far field $\left\|\mathbf{r}_{s_{n}, r_{m}}\right\|$ is a constant $K$, and Eq. 6.2 is expressed as

$$
P\left(\mathbf{r}_{r_{m}}, \omega_{\mathrm{o}}\right) \simeq \frac{1}{K_{3 D}} \sum_{n=1}^{N} P\left(\mathbf{r}_{s_{n}}, \omega_{\mathrm{o}}\right) e^{j \frac{\omega_{\mathrm{o}}}{c}\left\|\mathbf{r}_{s_{n}, r_{m}}\right\|},
$$

where $K_{3 D}=4 \pi K$. However, this constant can be avoided in the equations taking into account that the directivity is expressed in relative terms. This simplification has the advantage of the formulation remaining the same in the 2D case, in a far field [Abramowitz and Stegun, 1970], where the constant changes to $K_{2 D}=4 e^{-j \frac{\pi}{4}} / j \sqrt{\pi \omega_{\mathrm{o}} K /(2 c)}$.

The summation of Eq. 6.3 can be expressed in matrix form as

$$
\mathbf{P}_{r}=\underline{\mathbf{C}} \mathbf{P}_{s},
$$

where $\mathbf{P}_{s}=\left[P\left(\mathbf{r}_{s_{1}}, \omega_{\mathrm{o}}\right), \ldots, P\left(\mathbf{r}_{s_{N}}, \omega_{\mathrm{o}}\right)\right]^{T}$ and $\mathbf{P}_{r}=\left[P\left(\mathbf{r}_{r_{1}}, \omega_{\mathrm{o}}\right), \ldots, P\left(\mathbf{r}_{r_{M}}, \omega_{\mathrm{o}}\right)\right]^{T}$, and then the matrix $\underline{\mathbf{C}}$ of Green's functions is defined as 


$$
\underline{\mathbf{C}}=\left(\begin{array}{ccc}
e^{j \frac{\omega_{0}}{c}\left\|\mathbf{r}_{s_{1}, r_{1}}\right\|} & \cdots & e^{j \frac{\omega_{0}}{c}\left\|\mathbf{r}_{s_{N}, r_{1}}\right\|} \\
\vdots & \ddots & \vdots \\
e^{j \frac{\omega_{0}}{c}\left\|\mathbf{r}_{s_{1}, r_{M}}\right\|} & \cdots & e^{j \frac{\omega_{0}}{c}\left\|\mathbf{r}_{s_{N}, r_{M}}\right\|}
\end{array}\right)
$$

In that problem, $\mathbf{P}_{r}$ is a known data: it represents the angular pressure distribution around the sources, according to the directivity diagram. Despite directivity diagrams being expressed in terms of absolute values and $\mathbf{P}_{r}$ being a complex number, this is not a handicap, as will be shown later in the results.

In general, Eq. 6.4 cannot be solved as $\mathbf{P}_{s}=\underline{\mathbf{C}^{-1}} \mathbf{P}_{r}$, because it normally corresponds to an under- or overdetermined system (the number of sources and the angular resolution, i. e., the number of receivers - which are not usually the same). In these cases, an approximate solution is usually obtained in a least squares sense. This can be carried out by means of the Minimum Least Square method, i.e. by means of the pseudo inverse [Rao and Mitra, 1971]. Eq. 6.6 shows the solution expression where matrix $\underline{\mathbf{C}}^{T} \underline{\mathbf{C}}$ is always square:

$$
\mathbf{P}_{s}=\left(\underline{\mathbf{C}}^{T} \underline{\mathbf{C}}\right)^{-1} \underline{\mathbf{C}}^{T} \mathbf{P}_{r} .
$$

It must be taken into account that the point source distribution affects the sound field resolution. For a proper solution, the distribution of point sources must comply with a relation between distances to properly synthesize the field. This relation depends on the frequency. In this way, it is possible to correctly synthesize a sound field between a maximum $f_{\max }$ and a minimum frequency $f_{\min }$ in a given distribution of sources [Williams, 1999]

$$
\begin{aligned}
f_{\max } & =\frac{c}{2\left\|\mathbf{r}_{s_{i}, s_{j}}\right\|_{\min }}, \forall i \neq j, \\
f_{\min } & =\frac{c}{2\left\|\mathbf{r}_{s_{i}, s_{j}}\right\|_{\max }}, \forall i \neq j .
\end{aligned}
$$

The application of the proposed algorithm to the different paradigms (FDTD, DWM, TLM, ...) is straightforward: pressure values $p(\mathbf{r}, t)$ are given into discretized spatial points $\tilde{\mathbf{r}}$ in a particular time step $t=n \Delta t$; in 
the sequel, $\underline{\tilde{\mathbf{r}}}_{S}$ and $\underline{\underline{\mathbf{r}}}_{r}$ must be considered as the discrete point position of sources and receivers (see Fig. 6.1(b)).

Once the pressure values $\mathbf{P}_{s}$ are calculated at the point sources (amplitude and phase), the discrete-time excitation signals must be achieved. For each point source of the array, when a sinusoidal signal with a given frequency $\omega_{\mathrm{o}}$ is employed, it is calculated by

$$
\left.p\left(\tilde{\mathbf{r}}_{r_{m}}, n\right)\right|_{\omega=\omega_{\mathrm{o}}}=\Re\left\{\left\|P\left(\tilde{\mathbf{r}}_{r_{m}}, \omega_{\mathrm{o}}\right)\right\| e^{j\left(\omega_{\mathrm{o}} n \Delta t+\angle P\left(\tilde{\mathbf{r}}_{r_{m}}, \omega_{\mathrm{o}}\right)\right)}\right\} .
$$

In case of a K-DWM/FDTD mesh (Eq. 4.38), this result can be directly incorporated into the equation as a source term just applying a scalar factor or as predefined pressure points [Schneider et al., 1998a], having transparent source in both cases. In a W-DWM, since this DWM approach assumes an initial wave shape, the result of Eq. 6.9 is included directly in the recursive iteration of this algorithm.

\subsubsection{Examples}

At this point, some examples of synthesis of directive sources are presented in order to validate the proposed algorithm.

\section{Simulation Set-Up:}

To carry out the following experiments, the K-DWM method has been selected because it is one of the most efficient methods. The Higdon method [Higdon, 1987] has been selected and implemented into the mesh boundaries in order to minimize the effects of the associated reflections.

In these examples, a 2-D mesh has been used. Simulations have been developed for two frequencies, 500 and $2000 \mathrm{~Hz}$. Due to the effects of the inherent dispersion in K-DWM, the sampling frequency $f_{s}$ has been selected as 20 times the maximum frequency to be simulated, $f_{s}=40 \mathrm{kHz}$. According to the Courant formula $(\Delta x=\sqrt{2} c \Delta t)$, each cell represents $0.012 \mathrm{~m}^{2}$.

The source points are distributed in a staircase circle, taking space sampling into account. The set-up is composed of 12 sources forming a circle with a radius of $0.096 \mathrm{~m}$ (around 8 cells of distance over the center point) for the frequency of $500 \mathrm{~Hz}$ and 12 sources for the frequency of $2000 \mathrm{~Hz}$ (around 30 cells of radius); this set-up is accomplished with Eqs. 6.7 and 6.8. This selection implies that the first distribution allows the synthesis of frequencies between approximately $450-900 \mathrm{~Hz}$ and the second distribution around 
1700-3300 Hz.

The receiver points have been placed in a circle with a $10 \lambda_{\max }$ radius around the central point in order to guarantee a far field conditions, where $\lambda_{\max }$ is the maximum wavelength of the synthesized field. A mesh of $500 \times 500$ cells has been used to accommodate the receiving points and leave some free space.

\section{Results:}

According to the mathematical development presented during the present section, the complex amplitude of the sources is calculated. Since the directivity diagram does not give any information about phase, a zero-phase or random-phase at receiver can be assumed, giving nearly the same results in absolute value terms. In all of the following experiments, a zero-phase has been considered for $\mathbf{P}_{r}$.

In the first example, let us consider a distribution of pressure for a given frequency $\omega_{1}=2 \pi 500 \mathrm{rad} / \mathrm{s}$ with an angular resolution of $\Delta \theta=\pi / 4$. The directivity data is defined in an anticlockwise manner as $\mathbf{P}_{r 1_{(\mathrm{dB})}}=[0,-3,-7$, $-4,-5,-10,-4,-1]$.

Fig. 6.2(a) shows, in the form of a continuous line, the expected directivity diagram and, in the form of a dotted line, the one obtained. A high level of agreement exists between the expected and the obtained results, and it is difficult to differentiate between the two. However, note that in the radiated sound field (Fig. 6.2(b)), sharp variations in the directivity between the eight data angles occur. When input information has a low angular resolution, this effect is more noticeable. When representing these results with a higher angular resolution, as shown in Fig. 6.2(c), some non-expected irregularities arise.

In cases with low angle resolution information, a possible solution could be to interpolate the original directivity in the unknown angles of the diagram, obtaining more angular resolution and then applying the algorithm. Fig. 6.3) shows the results obtained by increasing the angular resolution (5 degrees) and interpolating data with spline functions [Ahlberg et al., 1967]. It shows how the results follow a much smoother change and in a more expected way, as real sources. However, as expected, some differences exist between the results obtained with a lower and a higher directivity angular resolution, due to the characteristics of the Minimum Least Square Method. This is manifested as a directivity curve that does not pass exactly through 


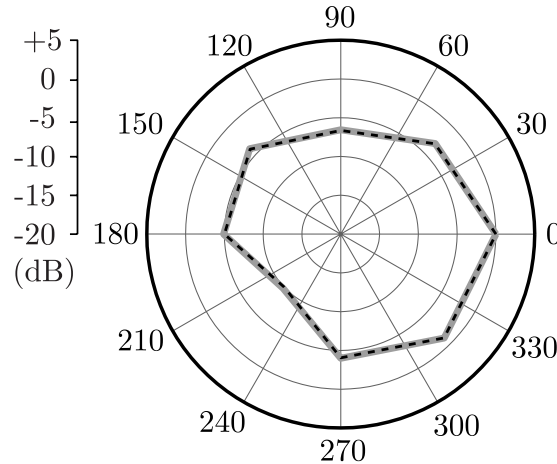

(a)

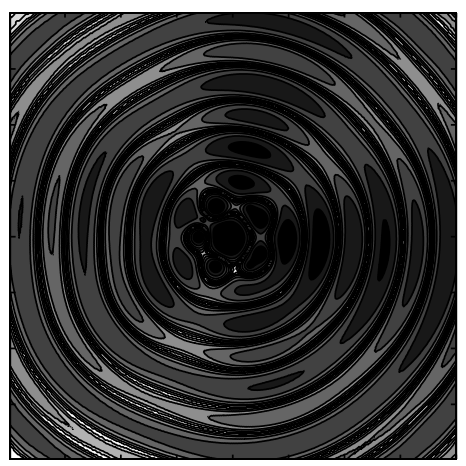

(b)

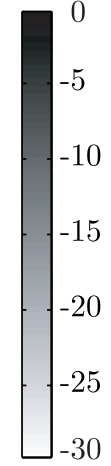

(dB)

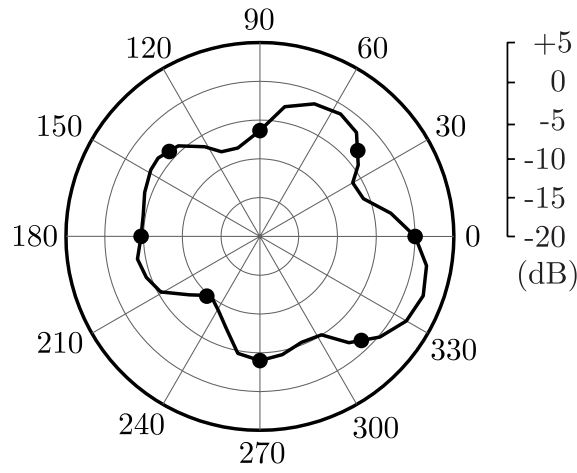

(c)

Figure 6.2. (a) Comparison between the expected (graycontinuous line) and the obtained (black-dotted line) diagram directivity in a discrete-time method. (b) Obtained radiated sound field simulated in a K-DWM mesh. (c) Obtained directivity diagram measured with a higher angular precision (dots indicate points used in the calculations) 


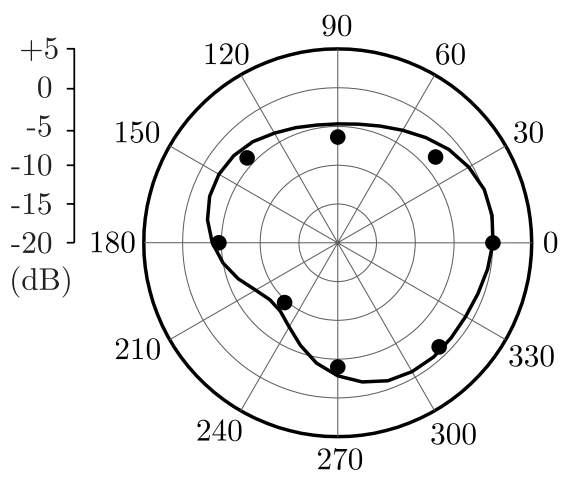

(a)

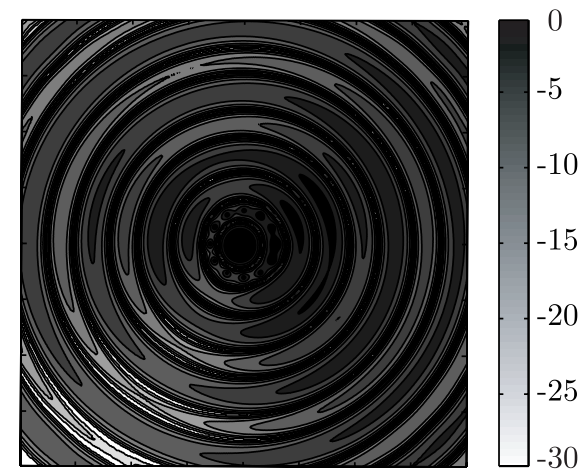

(b)

$(\mathrm{dB})$

Figure 6.3. (a) Directivity diagram obtained increasing the angular resolution by interpolation of the data of Fig. 6.2. (b) Obtained radiated sound field simulated in a K-DWM mesh.

the expected points of the original directivity diagram. There is a tradeoff between the smoothness of the curve and the precision at the reference points when the number of receivers increases.

It is also possible to find in the literature examples of directivity diagrams with a higher angular resolution. For instance in the next example, a directivity diagram of a baffled kettledrum is used [Fleischer, 1998], with a selected frequency of $500 \mathrm{~Hz}$, corresponding to the mode (31) (see Fig 6.4(a)). The number of sources is the same as the previous example (12 sources), but the angular resolution of the directivity diagram is 10 degrees (36 receivers). In Fig 6.4(a) the high similarity between the theoretical and the obtained directivity diagram can be observed and Fig 6.4(b) shows the sound field produced for the configuration of sources calculated by means of a K-DWM simulation.

One of the main advantages of discrete-time methods is their capacity to deal with broadband results, allowing several discrete frequency directivities to be carried out in a unique simulation. In order to show this, let us consider a directivity at two frequencies, one is $\mathbf{P}_{r 1}$ at $\omega_{1}=2 \pi 500 \mathrm{rad} / \mathrm{s}$, and the new one, for $\omega_{2}=2 \pi 2000 \mathrm{rad} / \mathrm{s}$, is $\mathbf{P}_{r 2(\mathrm{~dB})}=[0,-10,-12,-4,0$, $-6,-5,-2]$. The results in Fig. 6.5 show the sound field produced as the combination of both signals. This property allows the creation of complex 


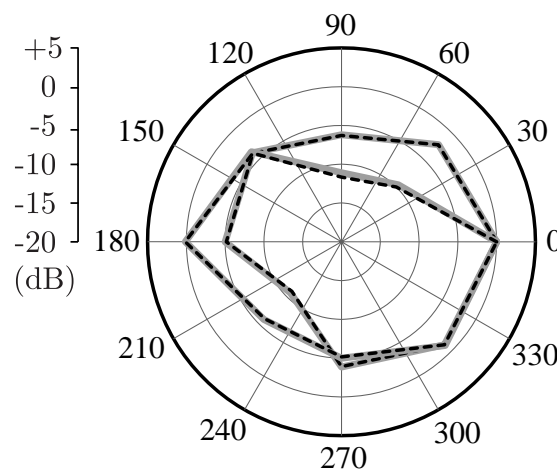

(a)

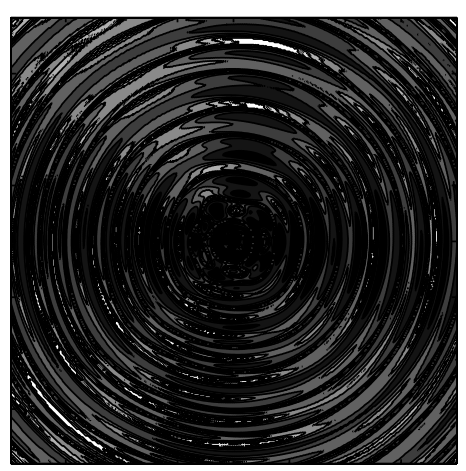

(b)

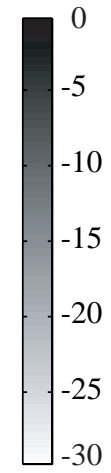

$(\mathrm{dB})$

Figure 6.4. (a) Comparison between the expected (graycontinuous line) and the obtained (black-dotted line) directivity diagram of baffled kettledrum in a discrete-time method. (b) Obtained radiated sound field simulated in a K-DWM mesh.

directive sources as a result of the combination of the single directivity diagram for different frequencies in a unique simulation.

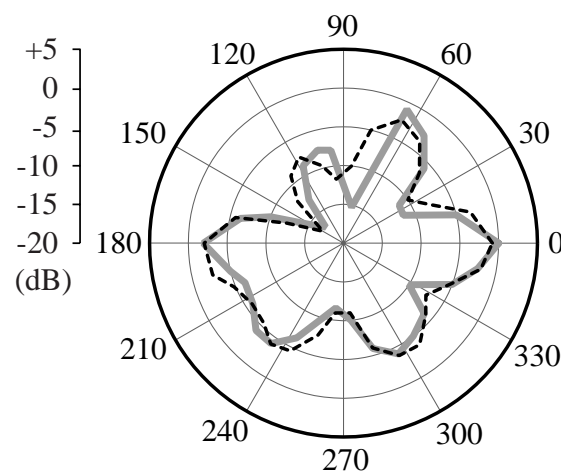

(a)

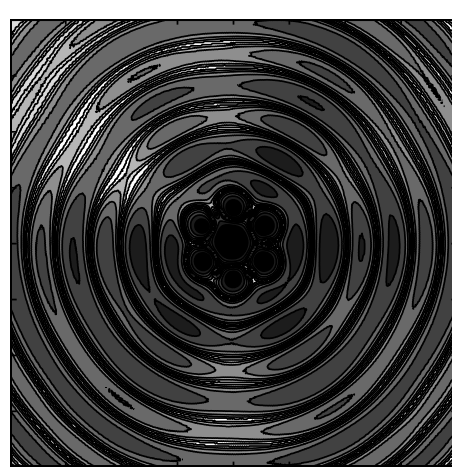

(b)

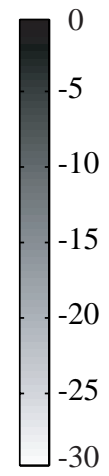

$(\mathrm{dB})$

Figure 6.5. (a) Comparison between the expected (graycontinuous line) and the obtained (black-dotted line) diagram directivity for two different frequencies in a discrete-time method. (b) Obtained radiated sound field simulated in a $K$ DWM mesh. 
It should be noted that the pressure sources mentioned in this work correspond to hard sources (the sources are not affected by the surrounding points). The influence in cases of transparent sources and realistic boundary conditions (partial reflections and frequency-dependent boundary conditions) should be analyzed.

\subsubsection{Influence of the mesh dispersion}

Results presented in this section have the scope of testing the same example in different meshes and to determine the viability of the proposed method as a function of the frequency and the dispersion of the mesh used. A short description of the dispersion properties of the methods appearing in this section can be found in Appendix A. For this purpose, common source positions are used: 12 monopoles, with a circular distribution and separated with an angle of 45 degrees. However, according to the theory presented, the frequency of the source is dependent of the separation between sources (Eq. 6.7 and 6.8). For that reason, the ratio of the source distribution will be selected in order to be able to correctly synthesize the sound field.

Results in all figures show the same directivity at different frequencies that have been shifted vertically $3 \mathrm{~dB}$ to elucidate the details, whereas amplitude scales have been suppressed purposely. The directivity vector used again is $\mathbf{p}_{r(\mathrm{~dB})}=[0,-3,-7,-4,-5,-10,-4,-1]$, which means that the directivity has an angular resolution of $\Delta \theta=\pi / 4$. The sampling frequency for all meshes is $40 \mathrm{kHz}$ and the spatial sampling frequency is determined by the specific condition that assures the mesh stability. The spatial sampling will be homogeneous for all directions. Considerations with the boundary conditions have been taken into account, truncating the temporal sequence before the reflected wave appears.

The results presented for each mesh represent the directivity for the frequencies $0.5,1,2,4$ and $5 \mathrm{kHz}$. It must be taken into account that as frequency increases, the distance between sources decreases. Then, there is a maximum synthetical frequency for a given temporal sampling frequency and hence, for the spatial sampling frequency. Additionally, in order to share the same criteria for all meshes, $5 \mathrm{kHz}$ has been determined as the maximum frequency for all simulations ${ }^{1}$. At the same time, each directivity

\footnotetext{
${ }^{1}$ It could be expected to analyze the directivity up to a higher frequency, i.e. a normalized value of $0.25\left(f / f_{s}\right)$ for the DWM. However, since not all the methods share the same cell size, the one with more restriction in terms of minimum distance between
} 

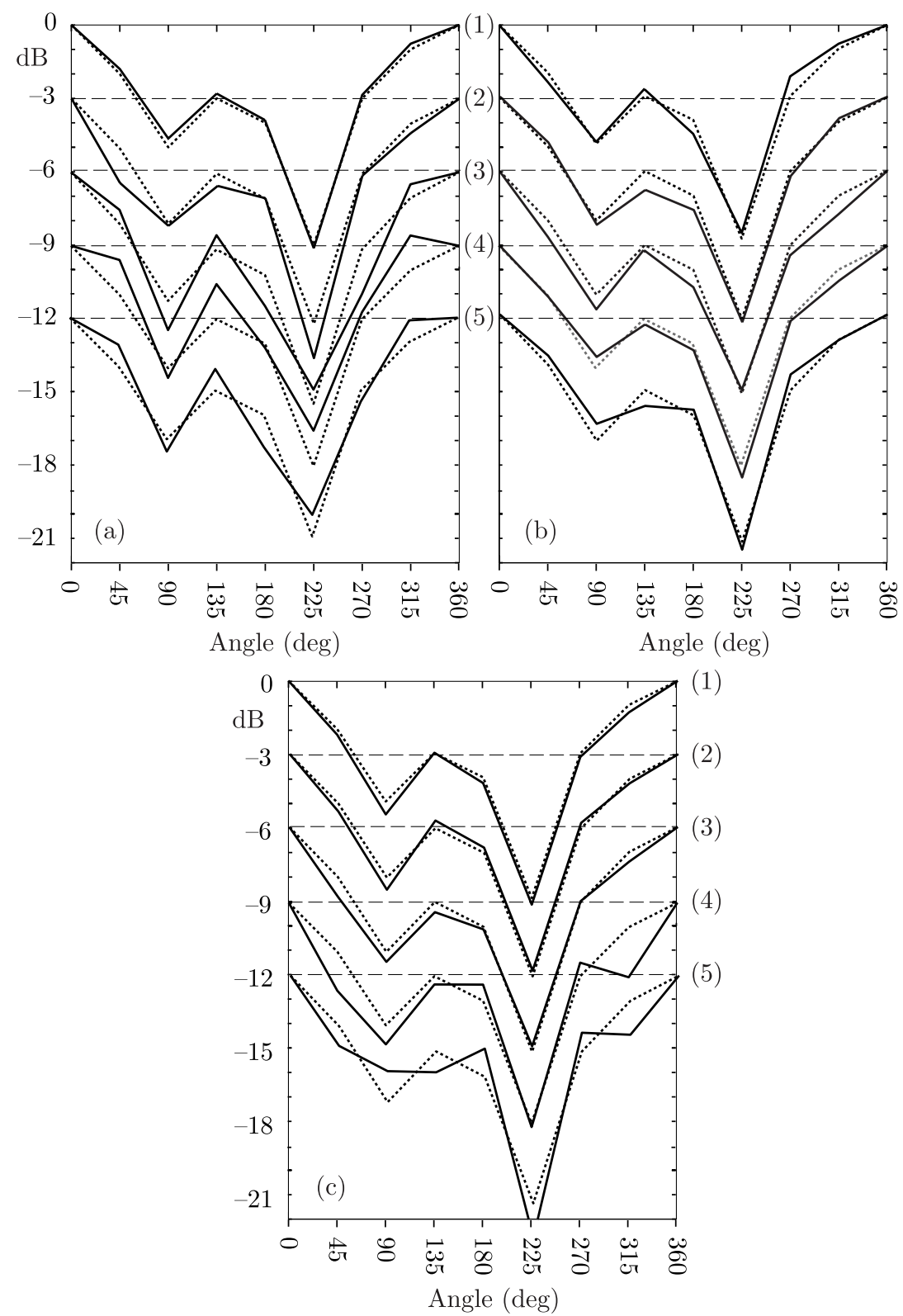

Figure 6.6. Directivity results for (a) DWM, (b) TDWM and (c) ULS. Dotted lines represent the expected results, whereas continuous lines are the result at the following frequencies: (1) $0.5 \mathrm{kHz},(2) 1 \mathrm{kHz},(3) 2 \mathrm{kHz}$, (4) $4 \mathrm{kHz}$ and (5) $5 \mathrm{kHz}$.Graphs are shifted vertically $3 \mathrm{~dB}$ to elucidate the details and amplitude scales are suppressed purposely. 


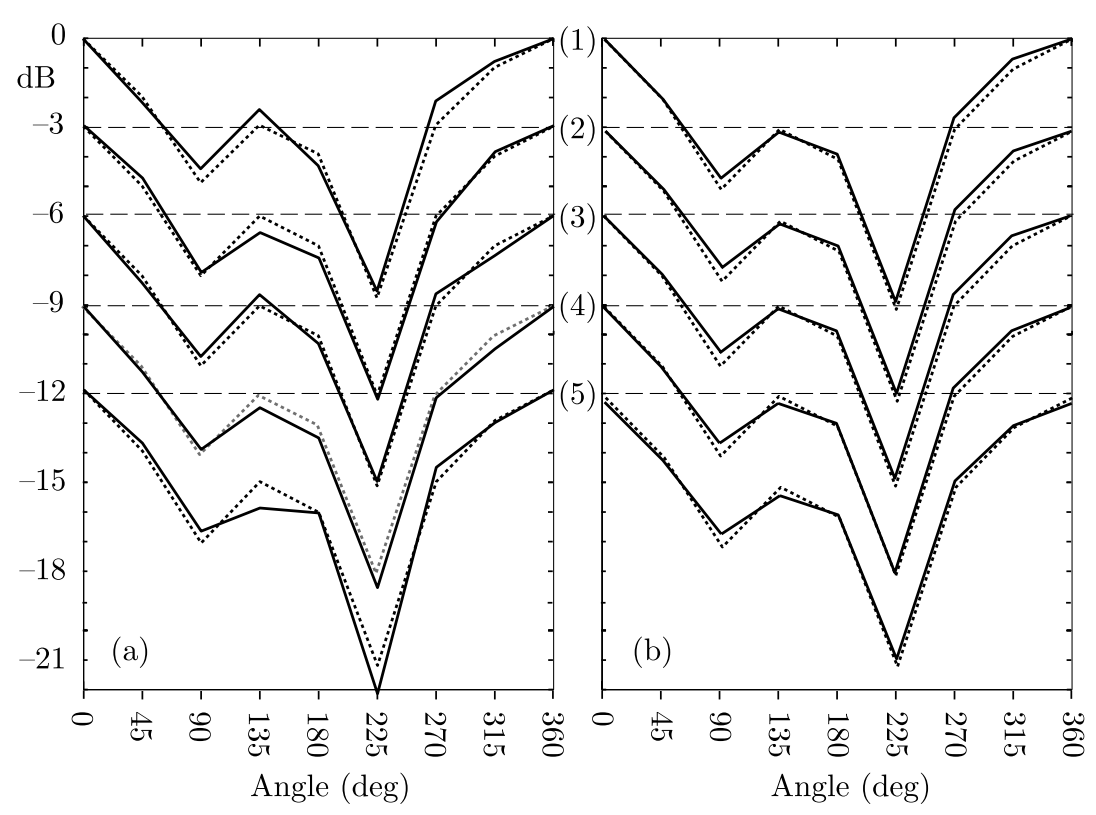

Figure 6.7. Directivity results for (a) Interpolated Digital Waveguide Mesh and (b) FTM. Dotted lines represent the expected results, whereas continuous lines are the result at the following frequencies: (1) $0.5 \mathrm{kHz}$, (2) $1 \mathrm{kHz}$, (3) $2 \mathrm{kHz}$, (4) $4 \mathrm{kHz}$ and (5) $5 \mathrm{kHz}$. Graphs are shifted vertically $3 \mathrm{~dB}$ to elucidate the details and amplitude scales are suppressed purposely.

(continuous line) is compared with the expected result (dotted line).

Figure 6.6(a) represents the results obtained with a K-DWM simulations, under the considerations specified above. The influence of the dispersion is notable. This dispersion is frequency and angle dependent and although the error is not excessive in the highest frequencies, the error compared to low frequencies is higher. In this example, the maximum error is around $2 \mathrm{~dB}$. However it can be observed how a significant dependence on the results exists with the frequency, where the directivity achieved increases its error when frequency grows.

cells should be chosen. In this particular case, the Upwind Leapfrog Scheme has a higher size for a given sampling frequency [Kim, 1997]. It allows synthesizing a directivity with a maximum frequency of around $0.125\left(f / f_{s}\right)$. 
As mentioned before (see Sec. 3.4.2), one of the most popular alternatives to reduce the dispersion in a DWM is the use of the Triangular DWM (TDWM). This dispersion is lower and the dependence on the angle is also quite lower. Fig. 6.6(b) shows the results of TDWM in which, compared to the results in a DWM mesh, a clear improvement can be seen. The maximum error obtained, that also appears at the highest frequencies, is around $1 \mathrm{~dB}$. It is also observed how the results keep approximately the expected shape, and no increasing errors seem to appear when frequency increases. This is because the TDWM remains mostly angular independent.

The use of the Upwind Leapfrog scheme has a clear advantage with regard to the decrease of the dispersion and the use of higher spatial sampling frequency. On the implementation of the directive sources, there is an advantage due the reduced dispersion, giving the results shown in Fig. 6.6(c), the errors are lower than in the case of the DWM implementation. However, due to the higher spatial sampling frequency for a temporal given sampling frequency, it allows synthesizing more compact directive sources at low frequencies, higher distance between cells, but the highest frequency is lower than that achieved in a DWM or TDWM mesh.

One of the most interesting results achieved in this work is related to the interpolated Digital Waveguide Mesh. As already stated, the main advantage of this mesh is that the dispersion has no angle dependence. Fig. 6.7(a) shows how the results are highly improved compared to the previous cited meshes. Even for high frequencies, the agreement with the theory is high. This agreement is because, although there is a phase error, it remains the same in all directions. This phase error does not affect the directivity pattern representation, usually given in absolute value. It can be observed how it is very similar to that obtained through the TDWM, since both methods propagate the wave almost evenly.

Finally, one of the most interesting properties of the FTM mesh for room acoustic simulation is due to its free dispersion, which has as a consequence the mesh with the lowest error. Fig. 6.7(b) shows how very low, even negligible error appears. It could be assumed, in this case, the error is more dependent on the minimum-least square method than that produced by the mesh itself.

These results show how the influence of the inherent mesh dispersion can affect the achievement of directive sources. However, it can be observed that the angular dispersion is more critical than frequency dispersion for this 
purpose, since error phase is constant with the angle. In summary, efficacy of the proposed algorithm is independent of the discrete-time method used.

\subsection{Broadband directive sources}

In Sec. 6.2, an algorithm for sinusoidal directive sources has been presented; then, just one frequency is simulated. However, real sources in room acoustics usually have a determined bandwidth and its directivity is not constant with the frequency. For that reason, it is necessary to modify the previous algorithm in order to synthesize broadband directive sources [Escolano and López, 2008].

For a given sampling frequency (the one used to discretize the mesh), let us consider a source with certain cut-off frequency ${ }^{2}$. The source bandwidth can be discretized in a uniform distribution of $L+1$ frequencies $\boldsymbol{\omega}=\left[\omega_{0}, \ldots, \omega_{l}, \ldots, \omega_{L}\right]$, where $\omega_{L}$ equals to $2 \pi f_{s} / 2$. The number of frequencies $L+1$ can be considered as samples of the Fourier Transform of a signal which would be the impulse response of a each source.

In a similar fashion to the sinusoidal algorithm, let us define Green's function matrix for specific frequency $\omega_{l}$ as $\underline{\mathbf{C}}\left(\omega_{l}\right)$

$$
\underline{\mathbf{C}}\left(\omega_{l}\right)=\left(\begin{array}{ccc}
e^{j \frac{\omega_{l}}{c}\left\|\mathbf{r}_{s_{1}, r_{1}}\right\|} & \cdots & e^{j \frac{\omega_{l}}{c}\left\|\mathbf{r}_{s_{N}, r_{1}}\right\|} \\
\vdots & \ddots & \vdots \\
e^{j \frac{\omega_{l}}{c}\left\|\mathbf{r}_{s_{1}, r_{M}}\right\|} & \cdots & e^{j \frac{\omega_{l}}{c}\left\|\mathbf{r}_{s_{N}, r_{M}}\right\|}
\end{array}\right) .
$$

For each frequency, a certain directivity in terms of the pressure at a far field position, $\mathbf{P}_{r}\left(\omega_{l}\right)$, is defined. Then, through a pseudo inverse of Green's matrix $\underline{\mathbf{C}}\left(\omega_{l}\right)$, in an equivalent way such as Eq. 6.6, the amplitud and phase of the sources, $P_{r}\left(\omega_{l}\right)$, is solved.

Once this process is repeated for each one of the frequencies given at $\boldsymbol{\omega}$, it is possible to define the next matrix

\footnotetext{
${ }^{2}$ Without loss of generality, a source could be assumed as low-pass filter.
} 
$\underline{\mathbf{P}}_{s}=\left(\begin{array}{cccccc}P\left(\mathbf{r}_{s_{1}}, \omega_{0}\right) & \cdots & P\left(\mathbf{r}_{s_{1}}, \omega_{L}\right) & P\left(\mathbf{r}_{s_{1}}, \omega_{L-1}\right)^{*} & \cdots & P\left(\mathbf{r}_{s_{1}}, \omega_{1}\right)^{*} \\ \vdots & \ddots & \vdots & \vdots & \ddots & \vdots \\ P\left(\mathbf{r}_{s_{N}}, \omega_{0}\right) & \cdots & P\left(\mathbf{r}_{s_{N}}, \omega_{L}\right) & P\left(\mathbf{r}_{s_{N}}, \omega_{L-1}\right)^{*} & \cdots & P\left(\mathbf{r}_{s_{N}}, \omega_{1}\right)^{*}\end{array}\right)$,

where in each file, new elements have been added in order to obtain a Hermitian vector ${ }^{3}{ }^{4}$ per file. Each row represents the solution of Eq. 6.6 for a given frequency $\omega_{l}$ and each file represents the spectrum of one of the monopole sources $\mathbf{r}_{s_{n}}, P\left(\mathbf{r}_{s_{n}}, \omega\right)$.

The next step is to apply the inverse discrete time Fourier transform on each one of the files, giving as a result the matrix

$$
\underline{\mathbf{p}}_{s}=\left(\begin{array}{ccc}
p\left(\mathbf{r}_{s_{1}}, n_{0}\right) & \cdots & p\left(\mathbf{r}_{s_{1}}, n_{2 L-1}\right) \\
\vdots & \ddots & \vdots \\
p\left(\mathbf{r}_{s_{N}}, n_{0}\right) & \cdots & p\left(\mathbf{r}_{s_{N}}, n_{2 L-1}\right)
\end{array}\right)
$$

where each file represents the impulse response of a source, $p\left(\mathbf{r}_{s_{n}}, n\right)$ and $n \in\left[n_{0}, \ldots, n_{l}, \ldots, n_{2 L+1}\right]$. These impulse responses allow a given original source $s(n)$ to be radiated with a particular directivity through the use of the convolution

$$
s\left(\mathbf{r}_{s_{n}}, n\right)=s(n) * p\left(\mathbf{r}_{s_{n}}, n\right) .
$$

The main advantage of this algorithm lies in the fact that different directivities for each one of the frequencies can be defined and then, to design complex sound sources. However, according to Eqs. 6.7 and 6.8, for a given source position distribution, it is only possible to synthesize a determined broadband. In the low frequencies, it is not really a handicap, since most of the real sources radiate omnidirectionally. It is also necessary to modify the sampling frequency of the calculated impulse responses (by over- or downsampling) in case it does not agree with the one of the simulation.

\footnotetext{
${ }^{3}$ Hermitian vector is a sequence $a_{k}$ with $k=0,1, \ldots, N$ satisfying

$$
\Im\left(a_{0}\right)=0 \quad \text { and } \quad a_{k}=a_{N-k}^{*} \quad \text { for } k=1, \ldots, N,
$$

where $\Im\left(a_{0}\right)$ denotes the imaginary part of $a_{0}$. It follows that $a_{n} / 2$ is real if $n$ is even.

${ }^{4}$ It says that the spectrum of every real signal is Hermitian. Due to this symmetry, all negative-frequency spectral samples of a real signal may be discarded and regenerate them later if needed from the positive-frequency samples.
} 
Some negative effects related to the circular nature of the discrete Fourier Transform could appear [Oppenheim et al., 1999]. In order to avoid those effects, impulse responses of the directivity filters should be long enough to assure they tend to zero at both sides (i.e., using an sufficiently high number of points in the FFT process).

This means the number of frequencies $L+1$ should be increased, by using an interpolation function. A window function can also be used, although it produces a loss in frequency resolution, but if one has an enough frequency resolution, this does not really matter.

\subsubsection{Examples}

In this section, several examples of the suitability of the method for broadband directivities are presented. The next simulations are obtained in a K-DWM, with a sampling frequency $f_{s}=40 \mathrm{kHz}$ in a 2-D mesh conformed by $900 \times 900$ spatial sampling. Let us consider a directivity pattern with an angular resolution $\Delta \theta=\pi / 4$ with $\mathbf{p}_{r(\mathrm{~dB})}=[0,-2,-5,-3,-4,-9,-3,-1]$. The first example consists of a constant directivity for a particular bandwidth. In order to synthesize this directivity, 12 sources homogeneously distributed in a circle shape are situated at a radius of $0.14 \mathrm{~m}$. This distance between sources, determines a bandwidth between 590 and $1500 \mathrm{~Hz}$. Figure 6.8 shows and compares the broadband results obtained. The results are presented normalized in respect to the pressure level obtained in direction 0ž. It can be observed how there exists a high agreement with respect to the expected. The highest differences could be found near to the highest possible frequency, but they are lower than $1 \mathrm{~dB}$.

Let us consider the same directivity pattern, but this time synthesized through a circle shaped source distribution situated at a radius of $0.058 \mathrm{~m}$. The bandwidth is now between 1.47 and $3.75 \mathrm{kHz}$. Figure 6.9(a) shows the results in a similar way to the previous one. However, it can be observed how, due to this example now working in a higher frequency range, some higher errors appear in the results. According to the results of Sec. 6.2.2, errors are more related to the angular dispersion, than due to the frequencydependent dispersion. A simple solution could be the use of the interpolated K-DWM, since it spreads the angular dispersion evenly (see Appendix A). Figure 6.9(b) shows the same example, but using the interpolated mesh. It can be observed how the results are improved and variations with respect to the theoretical references are decreased. 


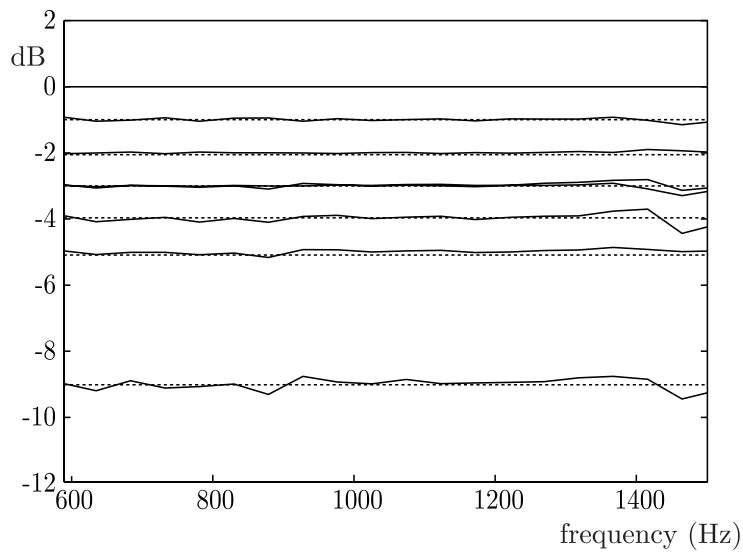

Figure 6.8. Broadband directivity results (solid line) obtained in a K-DWM grid. The dotted line represent the expected values.

In a similar fashion to the analysis of sinusoidal directive sources, it is necessary to check what happens in the rest of the directions. Figure 6.10 shows the same results as Fig. 6.8, but represented with a higher angular resolution $\Delta \theta=\pi / 36$. This means no modifications over the previous results, just that more angles are represented. It can be observed how a considerable number of lobes and noticeable transitions between the directivity of close frequencies appear, becoming unnatural. It can be seen how this apparition of lobes is more related to the Minimum Least-Square method than for the simulation process in Fig. 6.11 which represents the same results as in Fig. 6.9 (but also with more angular resolution), where (a) is the result with the conventional K-DWM method and (b) with the interpolated one. In a dynamical range which covers the maximum and minimum level of the variations with respect to the direction $0 \check{z}$, no relevant differences could be found between both simulations, demonstrating how the influence of the mesh characteristics (dispersion) does not affect the distribution of those undesirable lobes.

These lobulation effects appear when the directivity information is measured with a small angular resolution. A simple idea to solve these effects, already addressed in Sec. 6.2.1, is to interpolate the values at angular position, and then, to apply the proposed algorithm, having now a higher an- 


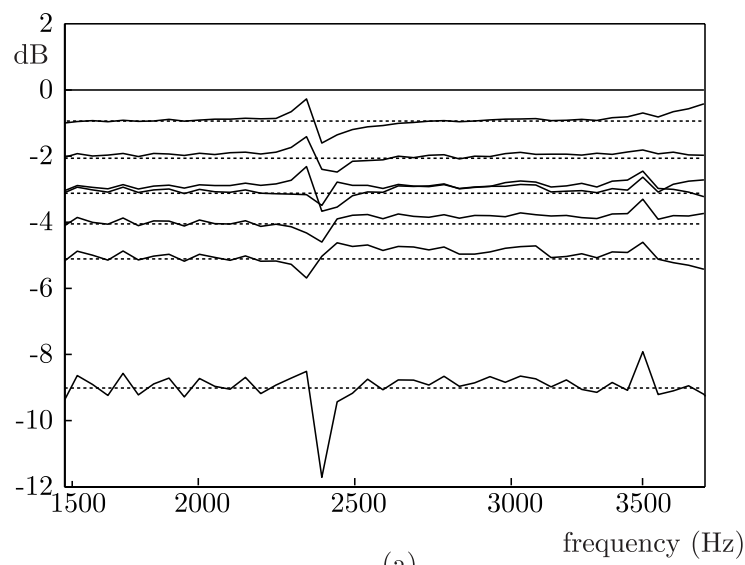

(a)

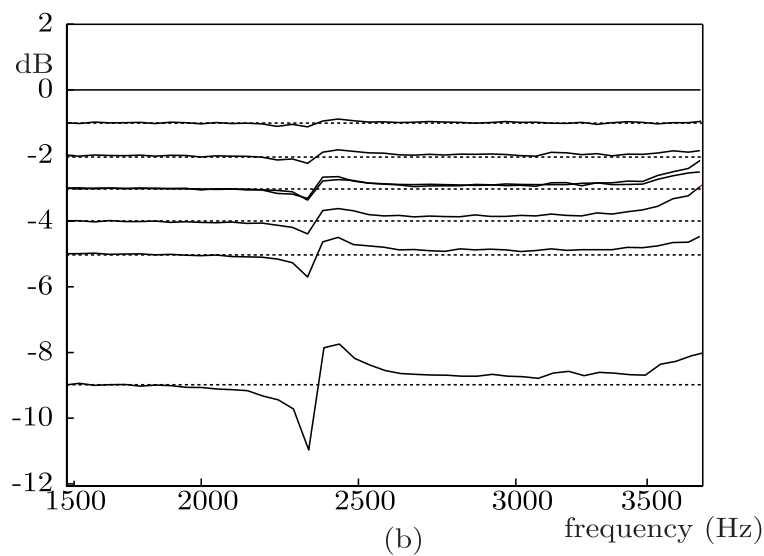

Figure 6.9. Broadband directivity results (solid line) obtained in a (a) K-DWM grid and (b) interpolated K-DWM grid. The dotted line represent the expected values.

gular resolution. Figure 6.12 shows the same directivity pattern as Fig. 6.8 after a spline interpolation with an angular resolution $\Delta \theta=\pi / 36$. The results show how the obtained directivity is now practically constant and no considerable differences can be observed, giving to the proposed method a considerable feasibility to define directivity sources in a K-DWM. It is straightforward to confirm this method is also applicable to the rest of discrete-time methods, such as FDTD, W-DWM or FTM.

The main advantage of this method lies on the fact that different di- 


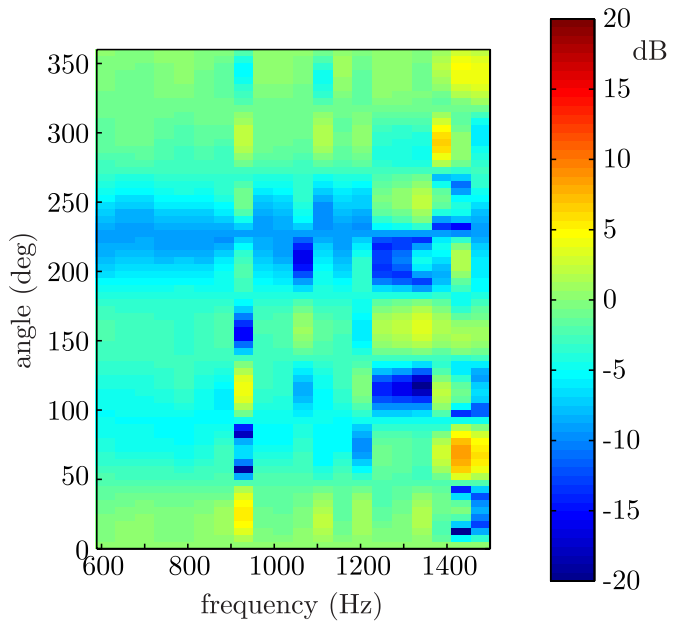

Figure 6.10. Same directivity results of the Fig. 6.8, measured with an angular resolution of $\Delta \theta=\pi / 36$.

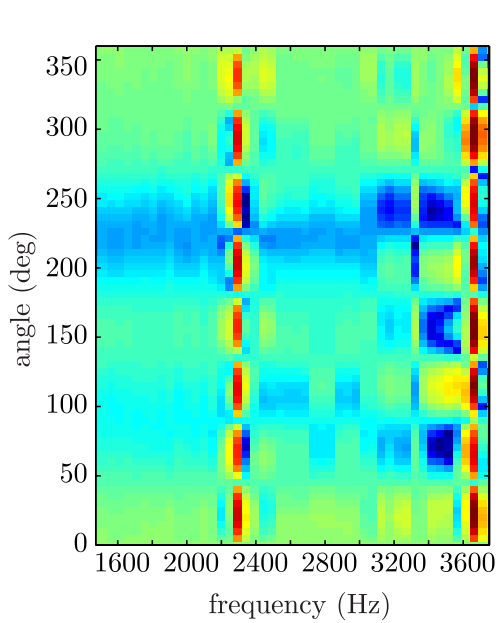

(a)

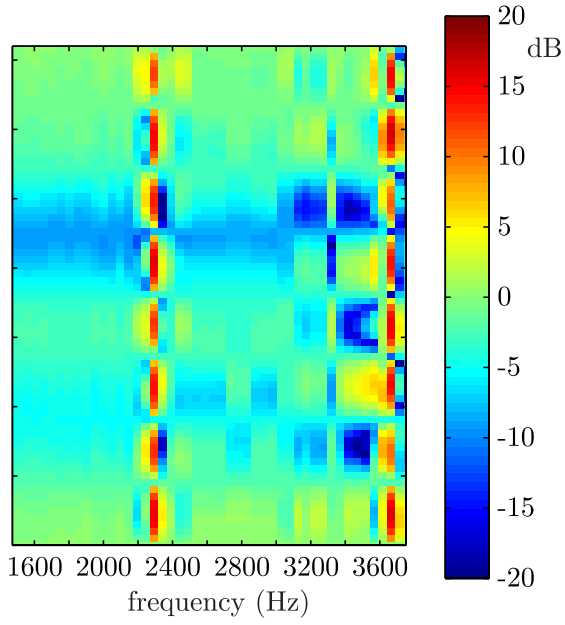

(b)

Figure 6.11. Same directivity results than Fig. 6.9, measured with an angular resolution of $\Delta \theta=\pi / 36$, for (a) a K-DWM and (b) interpolated K-DWM. 


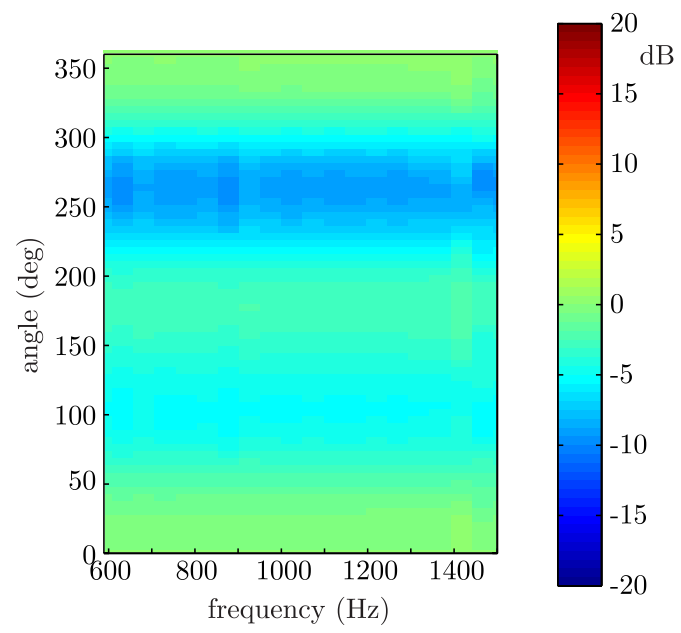

Figure 6.12. Same directivity results than Fig. 6.8 after a spline interpolation of the directivity pattern with an angular resolution $\Delta \theta=\pi / 36$.

rectivities at different frequencies according to the different measurements could be defined, including soft transitions between the original data. Let us consider an example: being a K-DWM grid with the same characteristics as the previous one but using the next directivity patterns: at a frequency $\omega=$ $2 \pi 500 \mathbf{p}_{r(\mathrm{~dB})}(2 \pi 500)=[0,-2,-5,-3,-4,-9,-3,-1]$; at a frequency $\omega=$ $2 \pi 500, \mathbf{p}_{r(\mathrm{~dB})}(2 \pi 1000)=[0,-2,-9,-3,-8,-1,-12,-5]$ and finally, at a frequency $\omega=2 \pi 2000, \mathbf{p}_{r(\mathrm{~dB})}(2 \pi 2000)=[0,-8,-10,-3,0,-5,-4,-1]$. Since the angular resolution is quite low, the directivities are interpolated through the use of spline functions in order to achieve $\Delta \theta=\pi / 36$. Furthermore, since the number of known of frequencies is also fairly reduced, the rest of the frequencies are interpolated, using 1024 frequency samples to represent the whole spectrum. This time, the interpolation function used is linear, since the splines could create elevated and unnatural values. The null frequency and the maximum frequency as omnidirectional have been also considered ${ }^{5}$.

Two source point distributions are used for synthesizing this frequency-

\footnotetext{
${ }^{5}$ Real sources are omnidirectional at low frequencies and directional at high frequencies; however, these values are selected in order to shown the feasibility of the method in the frequency range of the example.
} 


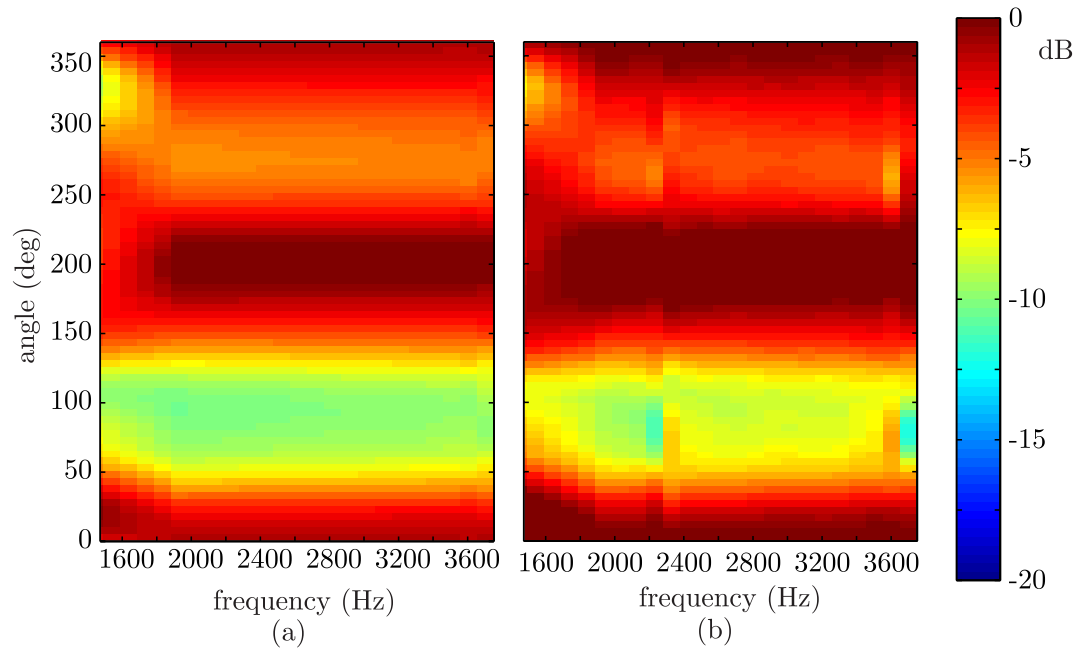

Figure 6.13. (a) Theoretical directivity pattern obtained through a circle shape source distribution with a radius $0.058 \mathrm{~m}$. (b) Same directivity results obtained through the proposed method in a K-DWM.

dependent directivity. Both distributions again consist of a circle shape source distribution, one with a radius of $0.058 \mathrm{~m}$ (between 1.47 and $3.75 \mathrm{kHz}$ ); and the other one with a radius of $0.14 \mathrm{~m}$ (between 0.59 and $1.5 \mathrm{kHz}$ ). Figure 6.13(a) represents the expected pattern using the circle of sources with a radius of $0.058 \mathrm{~m}$, whereas Fig. 6.13(b) represents the results. The elevated agreement between both graphics can be observed. Also, Fig. 6.14(b) shows the results with the other source distribution, which is compared to the theoretical directivity pattern. Again, the agreement is elevated. If each simulated directivity is subtracted from their corresponding expected pattern, an error distribution is achieved. Their corresponding error distributions are presented in Figs. 6.15 and the maximum error is estimated at no higher than $\pm 3 \mathrm{~dB}$, although these errors are located in a few points, the average error being nearly inappreciable.

It is also interesting to shown how the impulse response is propagated. Both source configurations are calculated to emit a gaussian pulse according this directivity. Figure 6.16 shows both mentioned source configurations at different time steps. It can be observed how impulse responses have 


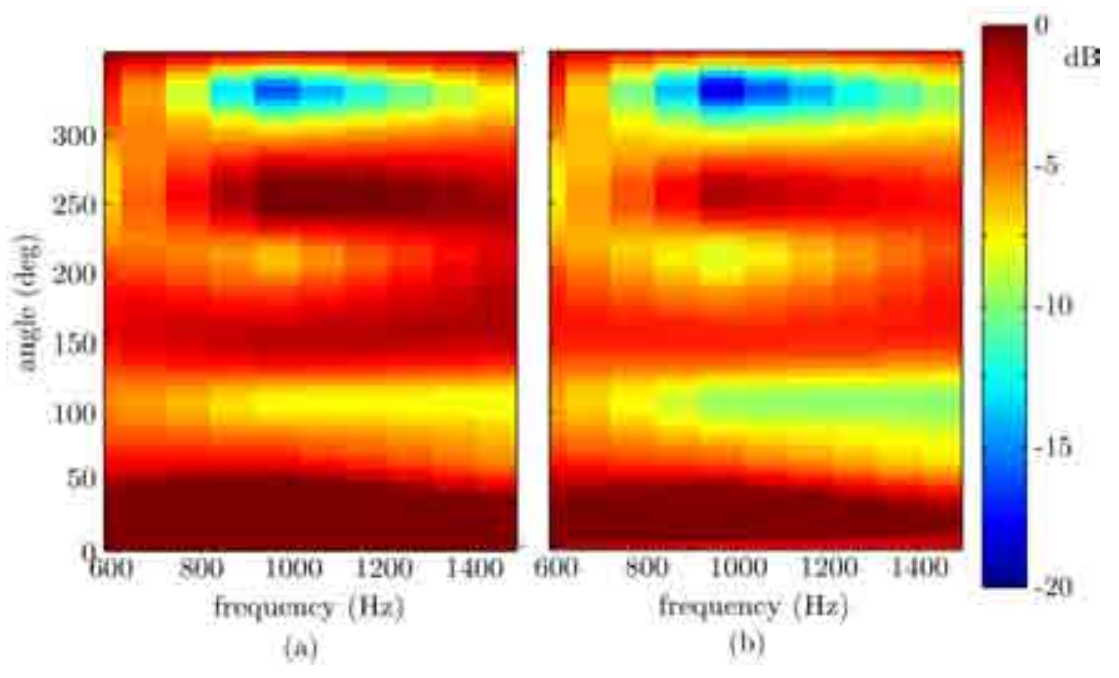

Figure 6.14. (a) Theoretical directivity pattern obtained through a circle shape source distribution with a radius $0.14 \mathrm{~m}$. (b) Same directivity results obtained through the proposed method in a K-DWM.

their gaussian shape when they reach a certain distance (far-field condition) and it is also noticeable the level difference as a function of the angle of propagation, being the directivity accurate in their corresponding frequency band, according to the source distribution used in each example. 


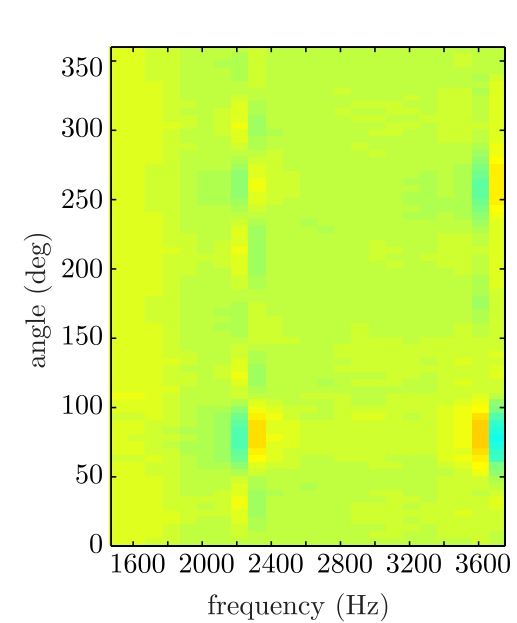

(a)

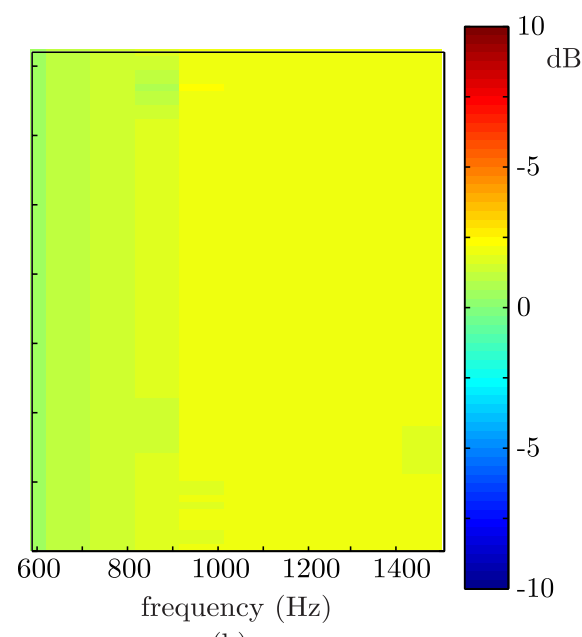

(b)

Figure 6.15. Error distribution obtained as the difference between the expected directivity and the results for a circle shape with radius: (a) $0.058 \mathrm{~m}$ and (b) $0.14 \mathrm{~m}$. 

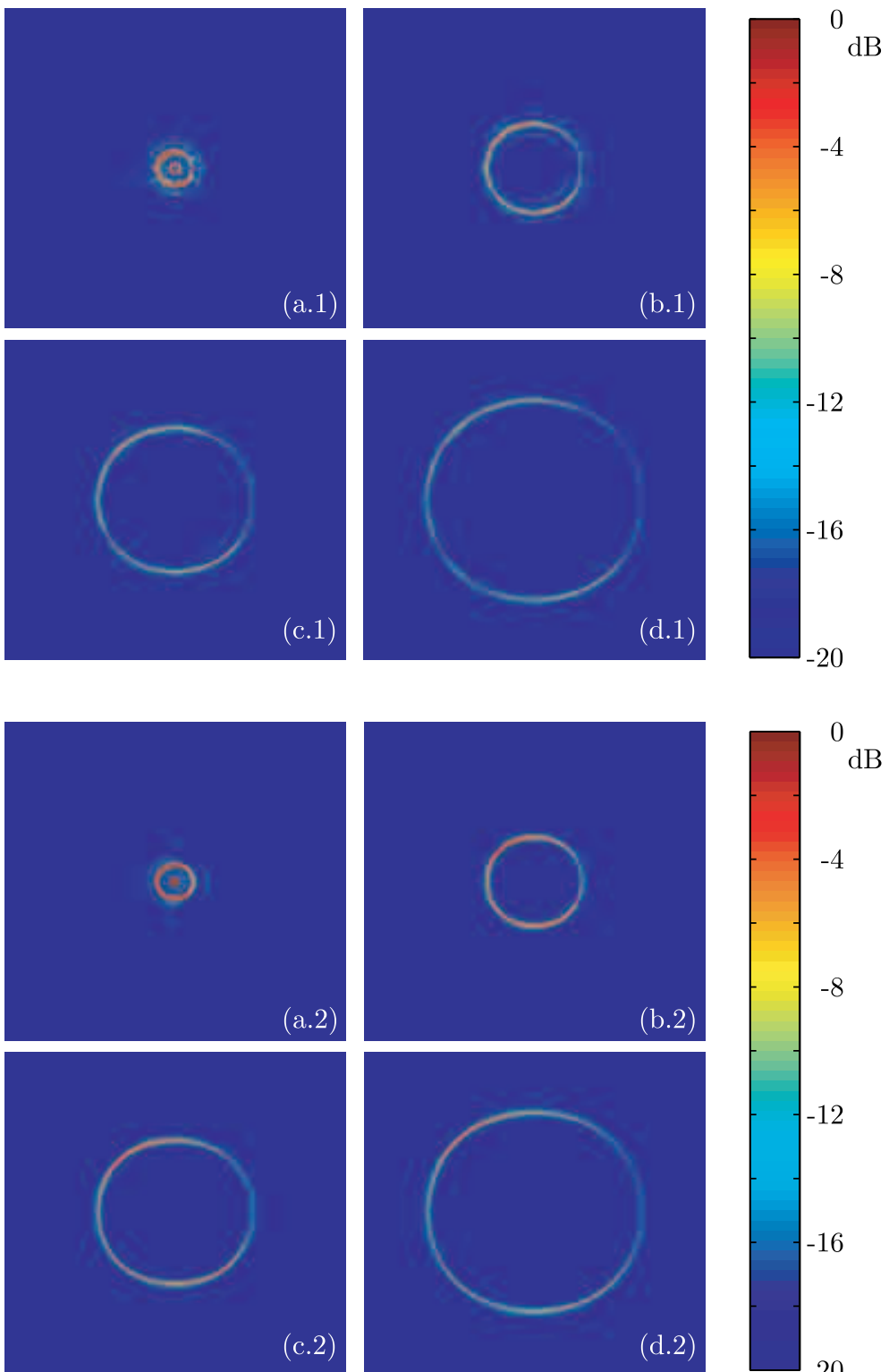

Figure 6.16. Impulse response generated by the directive sources for the source distribution in a circle shape with radius: (a) $0.14 \mathrm{~m}$ and (b) $0.058 \mathrm{~m}$. These impulse responses are presented at different time steps. 


\subsection{Discussion}

Directive sources in time-domain methods remain as an unsolved problem, and certainly, a very few attempts have been proposed. This should be an important concern in discrete-time methods for room acoustic simulation, since not only the position of the sources, but also their radiation patterns have a considerable influence in the total sound field obtained in a particular room. However, so far, the radiating sources in discrete-time methods are considered as point sources, e.g. monopoles, and this can produce an important lack of accuracy in the final result. Unfortunately, there is very little literature related to this topic for discrete-time modeling.

In this chapter, it has been demonstrated how it is possible to create a certain directivity pattern with the suitable combination of monopole. Given a certain monopole distribution, a directivity could be calculated considering amplitudes and phases of each monopole in order to obtain a particular far field pressure distribution. The first presented approach to this problem has only considered sinusoidal sources. Since this algorithm is based on the use of monopoles, it works for all those discrete-time methods. Differences in the final results are strongly influenced by the dispersion of each of these methods, being angular dependent dispersion the more decisive parameter.

Although the achieved directivity using the proposed method for sinusoidal sources gives an accurate solution, this method itself is not enough for real simulations, since sources have a width spectrum. For that reason, it should be extended for broadband sources.

From the basis of this method, an extension to a broadband frequency directivity pattern has been presented. For this method, the goal is to calculate a suitable impulse response of each monopole, obtaining not only accurate results but also smooth transitions between directivities for consecutive frequencies. 


\section{Conclusions and Future Research Lines}

Calvin: I think we've got enough information now, don't you?

Hobbes: All we have is one "fact" you made up. Calvin: That's plenty. By the time we add an introduction, a few illustrations and a conclusion, it will look like a Ph.D thesis.

(Yukon Ho!, B. Watterson, 1989)

As concluding remarks, a brief description of the conclusions and contributions of this thesis is presented, referencing the journal articles and conference papers that have been published from this work, as well. Finally, some proposed guidelines for future research lines are indicated (some of them have already started).

\subsection{Summary and conclusions}

Despite there existing a firmly established acoustic theory in room acoustics, it is not possible to find analytical expressions able to describe the whole acoustic phenomena occurring in a room as a function of position and time, see Chap. 2. For that reason, some simplifications should be applied to this room acoustic theory in order to perform computer simulations. 
These computer simulations have a considerable interest for engineers and architects, and also for the entertainment industry, as well as virtual reality applications.

Room acoustic simulation, as treated in this thesis, comprises the algebraical method approach to solve the inhomogeneous wave equation, and the way to define the boundary conditions. the ones which characterize the scenario under analysis. Depending on which method is selected to perform the simulation, there exists a way to define those boundary conditions and sound sources, see Chap. 3.

In this thesis, the use of discrete-time modelling is justified since it allows relatively efficient simulations with a high level of accuracy, since it corresponds to a discretized solution of the wave equation. Furthermore, defining these methods in the time domain, one only needs just one simulation to calculate the impulse responses of the entire room, see Chap. 4. And although a considerable number of advances and contributions exist to these methods of room acoustics during the last fifteen years, there is a notorious lack of solutions for some types of boundary conditions and sound sources modeling.

The main motivation of this thesis, has been to contribute to discretetime methods presenting algorithms to, on the one hand, perform specular boundary conditions in a locally reacting impedance sense, see Chap. 5; and on the other hand, how to define directive sources, see Chap. 6.

Regarding boundary conditions, this thesis deals with the locally reacting impedance concept, meaning impedance value is fixed and its value is independent of the plane wave angle of arrival, which simplifies considerably the boundary definition. The main trend is to describe the wall effects using the W-DWM and of plugging in a digital filter representing a reflection factor. However, this is not accurate, since the reflection factor is dependent of the angle of arrival and, in order to simplify computer complexity, a normal incidence is assumed. In order to improve this method, two techniques are proposed: first, to detect the angle of arrival in each iteration and calculate a suitable reflection factor filter for that time step; or as a second option, to plug in an impedance filter, which is independent of the angle of arrival. According to the results, this second option gives the most accurate solutions with a considerable small computational cost, and then, it arises as the most interesting solution according with its low complexity requirements. In a similar fashion to this second option, an algorithm for locally reacting 
impedances in the FDTD method is also proposed. In order to validate the results obtained through the contributions of this thesis concerning to the boundary conditions, the results are compared with analytical expressions derived from a semi-empirical equation of a hard-backed layer of porous material impedance.

Considering the sound sources/excitation modeling so far, point sources have been commonly used in these simulation methods; however, real sound sources do not spread their acoustic power evenly and the final pressure distribution depends not only on the source positions but also on their spread characteristics. In this thesis, a suitable distribution of monopoles (point) sources is used to perform a given directivity pattern. This method, although initially proposed for sinusoidal sources, has also been extended to broadband sources. The main advantage of this proposed method lies in its ability to represent a frequency-dependent directivities with considerable accuracy and even with smooth transitions between the different directivities at consecutive frequencies.

\subsection{Contributions of this thesis}

In this thesis, the main contributions can be highlighted as follows:

- A deep analysis of previous implementations for representing frequencydependent boundary conditions using the reflection factor in the DWM method is presented. This allows the identification and fully understand of its behaviour as a function of the plane wave angle of arrival and to verify how this corresponds with realistic boundary conditions, see Sec. 5.2.2.

- The main drawback of the boundary filtering used so far in the DWM method, lies in its lack of information about the angle of arrival, in order to realize the locally reacting impedance model. A method based on detecting this angle of arrival is proposed in order to improve the specular behaviour of the walls based on the use of reflection filters, see Sec. 5.2.3.

- An alternative and more accurate method for frequency-dependent modeling of boundary conditions is also proposed for the DWM method. 
This is based on the use of an impedance filter defined through Wave Digital Filters, instead of a reflection factor filter. Using this algorithm, the boundary conditions are modelled as a locally reacting impedance without any additional computational cost, see Sec. 5.2.4. It has been also compared with measurements in a real scenario, see Sec. 5.4.

- In a similar fashion to the previous contribution, an algorithm for an efficient implementation of a frequency-dependent boundary condition in the FDTD method is also proposed, see Sec. 5.3.

- Directive sources could be defined as a linear combination of point sources. An algorithm based on a least-squares method for reconstructing the acoustic pressure field is introduced for sinusoidal waves and then extended for broadband sources, see Sec. 6.2.

- Since the proposed method considers point sources, it is suitable for broader implementations beyond the FDTD and DWM methods. It has been analyzed in different methods and how the particular dispersion characteristics of each method could modify the simulated sound source distribution, see Sec. 6.2.2.

- However, since the numerical methods used in this thesis are based on the time domain, the full capacity of those methods for broadband simulation is not employed. Furthermore, real sources in room acoustics usually have a certain bandwidth and their directivity is not constant with the frequency. For that reason, the previous algorithm is extended in order to synthesize broadband directive sources, see Sec. 6.3.

Some parts of this program of research have been presented previously, at conferences and in journal papers. These publications are listed as follows:

\section{Journal articles}

- J. Escolano and F. Jacobsen, "A note on the physical interpretation of frequency dependent boundary conditions in a digital waveguide mesh", Acta Acust. united with Ac., 93(3), pp. 398-402, May/Jun, 2007. 
- J. Escolano, J. J. López and B. Pueo, "Directive sources in acoustic discrete-time domain simulations based on directivity diagrams", $J$. Acoust. Soc. Am., 121(6),pp. EL256-EL262, Jun, 2007.

- J. Escolano, F. Jacobsen and J. J. López, "An efficient realization of frequency dependent boundary conditions in a finite-difference timedomain model", J. Sound Vibr., 316, pp. 234Ü-247, 2008.

- J. Escolano, J. J. López and B. Pueo, "Broadband directive source simulation in discrete-time methods", J. Acoust. Soc. Am., (submitted).

- J. Escolano, J. J. López and B. Pueo, "Locally reacting impedance simulation in a digital waveguide mesh by means of mixing modeling strategies for room acoustic simulation", Acta Acust. united with Ac., (submitted).

\section{Conference articles}

- J. Escolano and J. J. López, "Broadband directive sources modeling for acoustic discrete-time domain methods" (abstract), J. Acoust. Soc. Am., 123(5), pp. 3798, May 2008.

- J. Escolano, J. J. López, B. Pueo and M. Cobos, "A note on the implementation of directive sources in discrete time-domain dispersive meshes for room acoustic simulation", 123th AES Convention New York, USA, Oct 2007.

- J. Escolano, B. Pueo, J. J. López and M. Cobos, "Empirical evaluation of the frequency-dependent boundary conditions in a Digital Waveguide Mesh", 124th AES Convention Amsterdam, Netherlands, May 2008.

- J. J. López, J. Escolano and B. Pueo, "Simulation of complex and large rooms using a Digital Waveguide Mesh", 123th AES Convention New York, USA. Oct 2007.

- J. J. López, J. Escolano and B. Pueo, "On the implementation of a room acoustics modeling Software using finite difference time domain methods", 122th AES Convention Vienna, Austria. May 2007. 
- J. Escolano and F. Jacobsen, "Physical interpretation of the frequency dependent boundary conditions in a digital waveguide mesh", 13th International Congress on Sound and Vibration (ICSV'06), Vienna, Austria, July 2006.

\subsection{Future research lines}

From the conclusions of this work, some new and challenging research lines could be proposed, being some of them already open. Future work may follow the lines listed here:

- To evaluate the proposed boundary condition algorithms in more efficient meshes, such as triangular and hexagonal DWM, and even in a warped-interpolated mesh. The current trend in room acoustic simulation is to use these types of meshes, since they considerably reduce dispersion and then, it is possible to use a lower oversampling factor. This makes them more suitable for room acoustic simulation.

- To analyze the effects due the use of multidimensional KW- converters [Murphy and Beeson, 2007] to plug-in the proposed boundary condition based on a impedance in K-DWM.

- At the end of the writing process of this dissertation, Prof. Karjalainen [Karjalainen, 2008] has published a journal paper which explores alternative methods to realize consolidated impedances through WDFs. Although the results obtained in this paper could improve the efficiency of the model proposed in this thesis, they have been not considered under a matter of time. However, the importance of these contributions may help to open new research lines.

- To include not only reflections, but also effects due to transmission loss for a locally reacting impedance, in order to simulate thin solid slabs, plates or blankets. Although in developing this thesis only reflection from walls was assumed, there are some cases where a substantial portion of the energy goes through the boundaries, and this transmitted energy modify the total sound field, i.e., curtains.

- Regarding the sound source modeling, since multipoles can be defined as a combination of monopoles situated close enough, a formu- 
lation based on multipoles decomposition through spherical harmonics should be introduced. It could provide a more compact formulation of directive sources and improve the directivity at higher frequencies.

- Since this thesis is mostly a theoretical approach to the boundary conditions problem and the sound source modeling in discrete-time methods, a next step will be to compare the results obtained with the proposed algorithms in simulated room, and then to evaluate the performance of these algorithms in real scenarios. Although a first approach to verify the accuracy of one of the proposed method for boundary conditions has been already presented, all the proposed algorithms should be compared with measurements in real rooms, and then to determinate how close those simulations are to real world situations.

- To develop an end-user software, suitable to perform room acoustic simulations, including multi-process of parallelization. This is an active research and development line in order to provide the Waverb software [López et al., 2007a,b], which will include all the algorithms introduced in this thesis.

- To perform psychoacoustic evaluations of computed room acoustics which include these proposed methods: boundary conditions and directive sound sources. One of the final aims of these simulation algorithms is to provide a psychoacoustic aceptable result; then, it should be demonstrated there are no artifacts in these algorithms which could be perceived as an anomaly in the listening to these simulations. 


\section{A note on the dispersion of some discrete-time methods}

In this appendix, a brief note about some of the most used discrete-time methods for room acoustic simulation is presented focusing on their dispersion characteristics. In this thesis, dispersion has been defined as the difference between the theoretical and the resulting speed of sound during the simulation. It use to be an angle and frequency dependent function, specially affecting high frequencies.

It is not the intention of this appendix to deal with the mathematics behind the dispersion analysis of these methods, but to present some simulations. Some references addressing a deeper analysis of the methods' mesh dispersion are included in this appendix.

In Fig. A.1, the same gaussian broadband point source has been simulated through the different methods and represented in a logarithmic scale from 0 to $-150 \mathrm{~dB}$, where $0 \mathrm{~dB}$ has been specified as the maximum level of the propagated signal:

- Digital Waveguide Mesh: The formulation of the dispersion of this method has been detailed in Sec. 4.2.1. Dispersion of the (K- and W-) DWM is represented in Fig. A.1(a). It can be observed how dispersion is maximized in the cartesian directions, whereas is minimized in the diagonal direction, establishing an evident angular dependence (see 


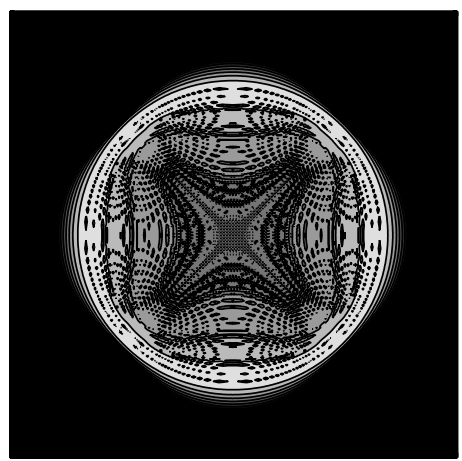

(a)

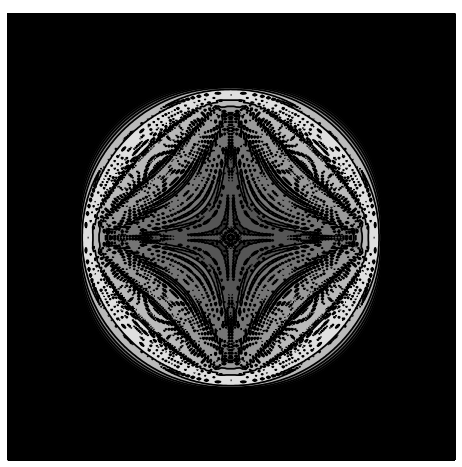

(c)

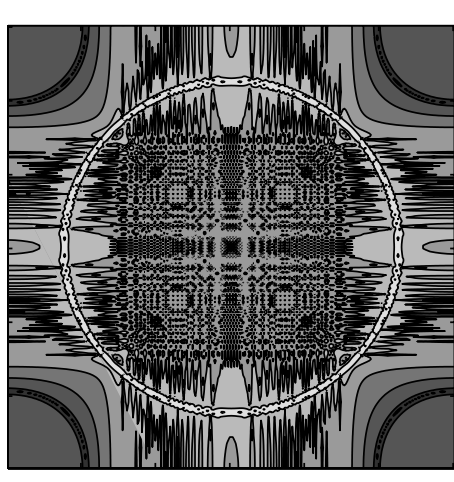

(e)

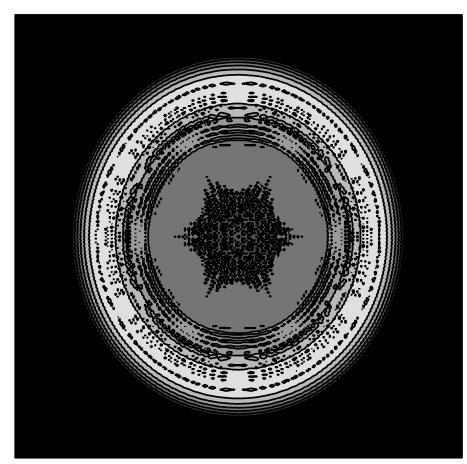

(b)

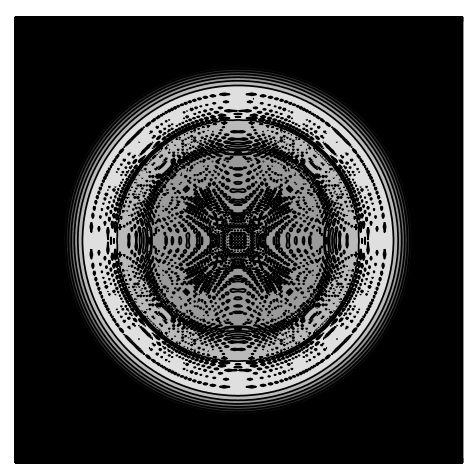

(d)

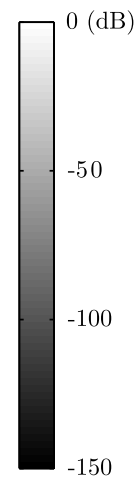

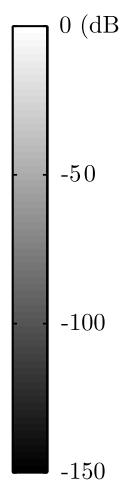

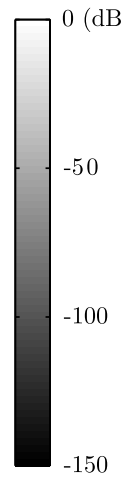

$-150$

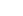


Ref. [Murphy, 2000, Duyne, 2007] for more details.).

- Triangular Digital Waveguide Mesh (TDWM): In Sec. 3.4.1, TDWM has been cited as an alternative DWM structure proposed in order to reduce the dispersion of the cartesian DWM. Figure A.1(b) shows an example of an implemented TDWM and the high reduction of the dispersion can be observed, even being almost not angle dependent. Accordingly [Fontana and Rocchesso, 1995], TDWM exhibits a high frequency dispersion error not exceeding $15 \%$ along all directions, while the cartesian DWM has almost $30 \%$ of error along the axes in high frequencies. A detailed comparison between the triangular and cartesian dispersion has been reported in Refs [Campos and Howard, 2005, Hacıhabiboğlu et al., 2008a]

- Upwind Leapfrog Scheme: This method has a more compact stencil compared with the classical leapfrog scheme for the FDTD/DWM method. Clustering the stencil around the preferred directions (characteristics), it enables high accuracy with a low order of operations. The most important advantages are related to the increment of the cell size for a given sampling frequency in respect to the classic leapfrog scheme of the FDTD method, and the reduction of the error dispersion (see Fig. A.1(c) for details). A detailed analysis of the dispersion can be found at Refs. [Thomas, 1996, Kim, 1997].

What is observed is a similar result to the DWM simulation, but the minimum dispersion occurs on the cartesian axis, whereas it is maximized along the diagonal directions. However, as cited in Sec. 3.4.2, the main advantage of this method lies in the efficiency of the method compared with the original FDTD/DWM scheme.

- Interpolated Digital Waveguide Mesh: The scope of this technique is to decrease, at least, the angular dispersion of a DWM [Savioja and Välimäki, 2003]. It takes into account the values of the cells not only in the cartesian directions, but also in the diagonal directions through suitable weight factors. This method does not reduce the dispersion substantially, however, its dispersion has (almost) a non angular dependence (see Fig. A.1(d)).

This mesh is not usually used by itself; it requires a pre- and postprocess based on the use of warping filtering [Savioja and Välimäki, 2003](d). 
- Functional Transformation Method: Some details about this method have appeared in Sec. 3.4.2. One of the main advantages of this method is that it is free of dispersion, as illustrated in Fig. A.1(e). Although this free dispersion characteristic has been reported by the authors [Petrausch and Rabenstein, 2005a], it can be observed some artifacts not reported so far. However, these artifacts appear since $50 \mathrm{~dB}$ below from the signal reference. 


\section{Software implementation}

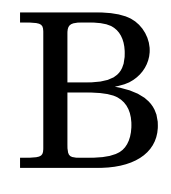

In this appendix, some considerations about the develop of computer software for room acoustic simulation based on discrete-time methods is presented. Although a front-end software is far from the scope of this thesis, some of the first efforts to develop the Waverb software [López et al., $2007 \mathrm{a}, \mathrm{b}]$ is presented, a DWM-based software which would include the contributions of this thesis. It should be noted from the literature, with the exception of the Roomweaver software [Beeson and Murphy, 2004] (now renamed as RenderAIR [Murphy et al., 2008a], developed in the Intelligent Systems Group/ Audio Lab, University of York, UK ${ }^{1}$ ), there is no other front-end software based on discrete-time methods for room acoustic simulation. This appendix cannot be considered as a contribution itself, but also it provide some guidelines followed in order to start with the development of a front-end software. These guidelines concern about some aspects such as the mesh definition and computational considerations.

\section{B.1 Mesh definition}

\footnotetext{
${ }^{1}$ URL: http://www.elec.york.ac.uk/intsys/
} 
A meshing algorithm takes the 3D model defined by its faces and vertex and maps it inside mesh composed by the cells that conform the object (in this case, room interiors). In the last few years, different algorithm variations have been attempted [Srisukh et al., 2002, Su et al., 2004] for the same topic. They are designed for the objects used in electromagnetism, but there are no references in the acoustic field. This algorithm presented here is based on those previously published works, but in the case of large and complex enclosures, special care and consideration has been taken to produce the models successfully.

The algorithm has different stages:

- Import of the CAD file: Loads the vector object from the CAD file and stores faces and vertex in the corresponding data structure. So far, the present software only imports $\mathrm{OBJ}^{\circledR}$ files, but any file can be employed by means of a format conversion using any available software.

- Boundary box: The object is scanned in order to accommodate the small boundary box. This is important because the size of the matrix variables that will contain the acoustic variables will have a size directly related to the box, and the memory has to be allocated. Through the Courant formula (see Eq. 4.35), cell sizes are obtained, and thus, the matrix size of the mesh is $N_{x} \times N_{y} \times N_{z}$. The most important role of the meshing algorithm is the identification and classification of interior cells on the object. This is made in the two next steps:

1. Plane section: The procedure to find such cells needs to simplify the 3 -D to a 2-D problem. For this purpose, an object intersecting process is performed. This is done via a series of intersecting perpendicular planes to one of the axis with a $\Delta \tilde{\mathbf{x}}$ (or $\Delta \tilde{\mathbf{y}}$ either $\Delta \tilde{\mathbf{z}}$ ) increment. Fig. B.1(a) shows a visual example of the procedure, where the $z$-axis has been chosen for the commented cuts, although any other axis would be valid. For this process, it is necessary to evaluate the eventual cut of such as plane with all sides of the object. If the plane intersects only one side, the cut segment is stored in a list. Once the process is finished, the intersection resulting polygon is configured. 
2. Polygon Filling: The procedure to obtain the inner polygon cells is a classic procedure of computer graphics. Basically, a ray scanning is performed, where a single ray, parallel to the $x$-axis, is traced for each $\Delta \tilde{\mathbf{y}}$, thus obtaining the cuts with the polygon. The result of the presented process is shown in Fig. B.1(c). Such an example is very simple, but there can eventually be objects presenting concavities or in the cut, there would appear more than a single polygon. For these situations, it is necessary to count the number of rays that cut on the polygon in such a way that when the number is odd, the cell is outside the polygon, whereas when it is even, the cell is inside. In Fig. B.2, the $r_{1}$ ray intersects the polygon three times so the cell is inside the object. The same occurs for $r_{3}$ ray since it only cuts once. On the contrary, $r_{2}$ ray lays within the object, as shown in Fig. B.2.

- Boundary cells identification: During the process of plane sectioning and polygon filling the cells are labelled as pertaining or not to the object. In order to take into account the boundary conditions, it would be desirable to label the cells in the boundary as a third category, in a reference to those boundary cells which are in contact with the air. This would help in the DWM recursion process when applying boundary condition algorithms. During the filling process, it is not possible to find all the cells in the boundary so a scanning process in the three axes is carried out. This process can find every boundary cell, even in concave volumes, or volumes with holes.

\section{B.1.1 Verifying the mesh modeling}

DWM code users need to be able to view and inspect their models after the meshing process to verify that the resulting grid accurately represents the object under study. Sometimes the CAD model may present small errors, such as tiny holes on the surface because of CAD design faults and some rays may escape in the scanning process. A visual verification of the mesh is very advisable to avoid such little errors that may ruin the process. To add this function, the developed software has the option to generate a file that contains the 3D mesh model that can be loaded with any 3D viewer of CAD software. To create a $3 \mathrm{D}$ verification file, one must proceed so:

- Scan the generated mesh, looking for cubes that pertain to the edge 


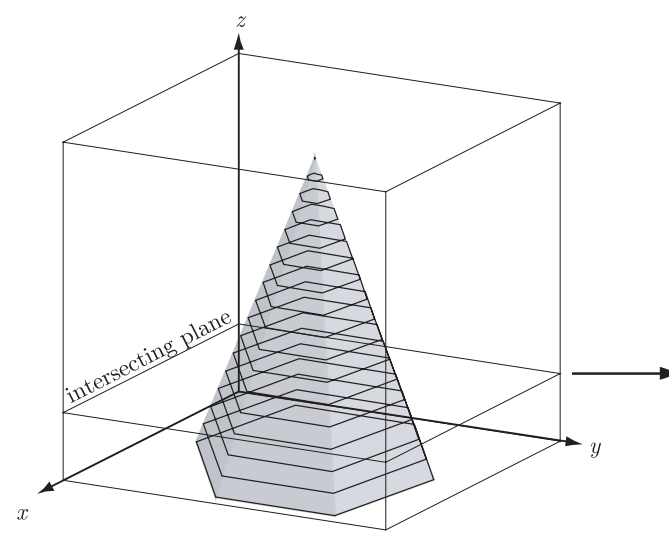

a)

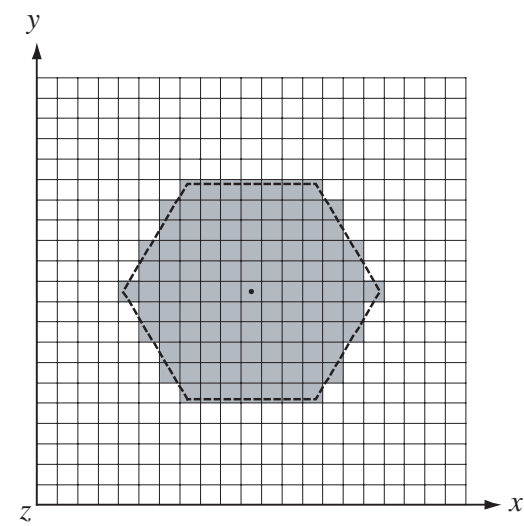

(c)

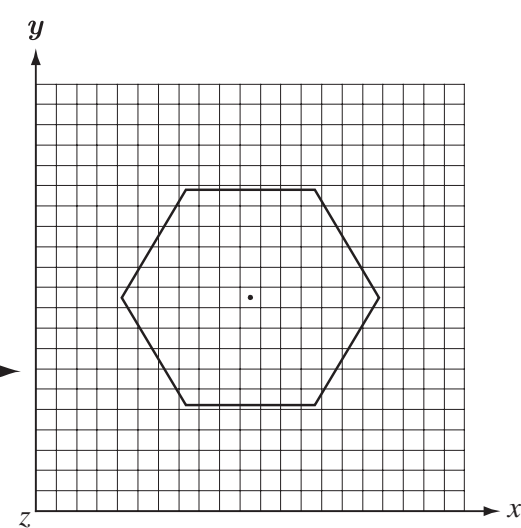

(b)

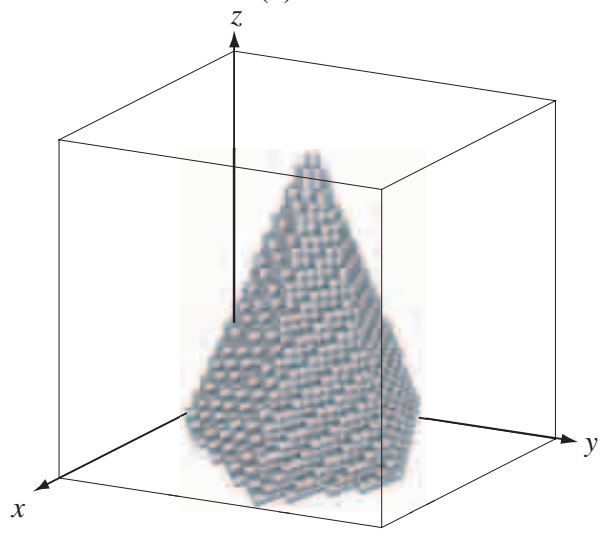

(d)

Figure B.1. Stages in the process of mesh generation.

(previously labeled).

- Generate the faces of each small cube, referencing to the correspondent vertexes.

- Save the face and vertex information according to the syntax of the selected fileformat (.OBJ in that case).

Fig. B. 3 shows and example exported by our software. It can be observed that the developed algorithm for meshing can manage objects with 


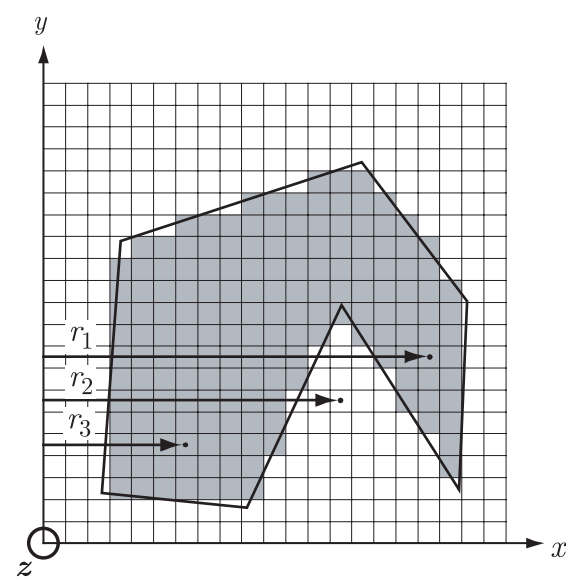

Figure B.2. Concave polygon filling procedure.

holes.

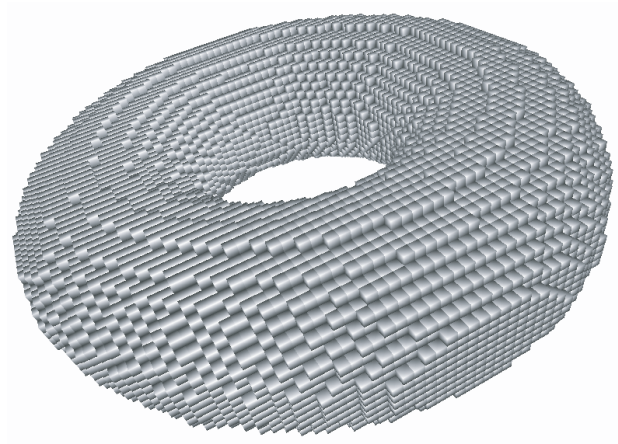

Figure B.3. Example of mesh with hole.

\section{B.1.2 Example}

In this section, some graphics concerning the meshing process when it is applied on a real room are presented. In this case, the CAD model belongs to the Rodrigo concert hall, in the Palau de la Música, Valencia (Spain) 
(see Fig. B.4). A more detailed view of this auditorium can be observed in Fig. B.5.

Figure B.6 shows a verification of the meshing process, where the cell size has been augmented for clarity.

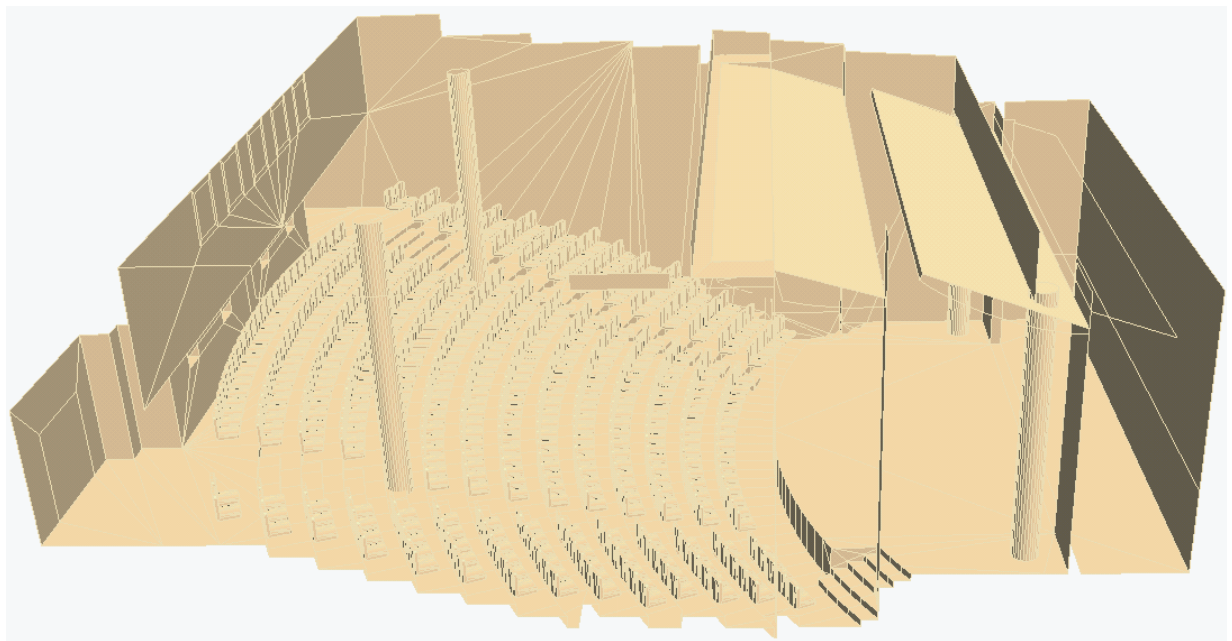

Figure B.4. CAD model of the auditorium.

\section{B.2 Graphical user interface}

The developed software so far, has a graphical user interface (GUI) in order to interact with the user, define all the parameters, execute the different tasks and show the status and the relevant information related to the simulation. Figure B.7 shows the main window of the GUI. It was decided to design a single screen in which to define the main parameters, instead of using different emerging windows that are hidden after each variation. This allows the user to always have a view of the main simulation parameters.

There are other windows devoted to more specific configurations, such as the management of the material database and the material index associated to the room geometry file that has been loaded.

The execution actions are represented by buttons on the left side of the 

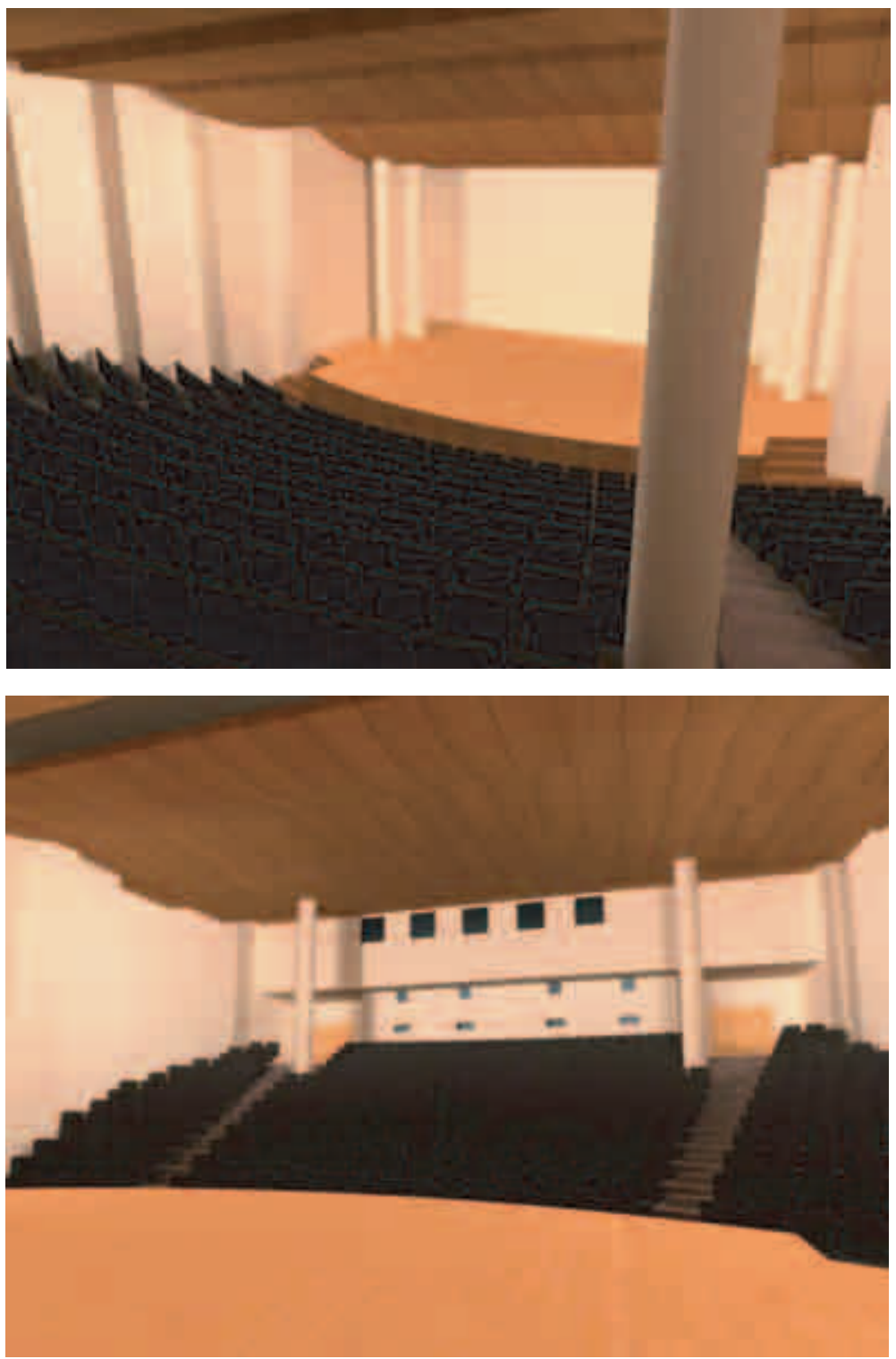

Figure B.5. Two views of the auditorium (stage and stalls) synthesized from the CAD model. 


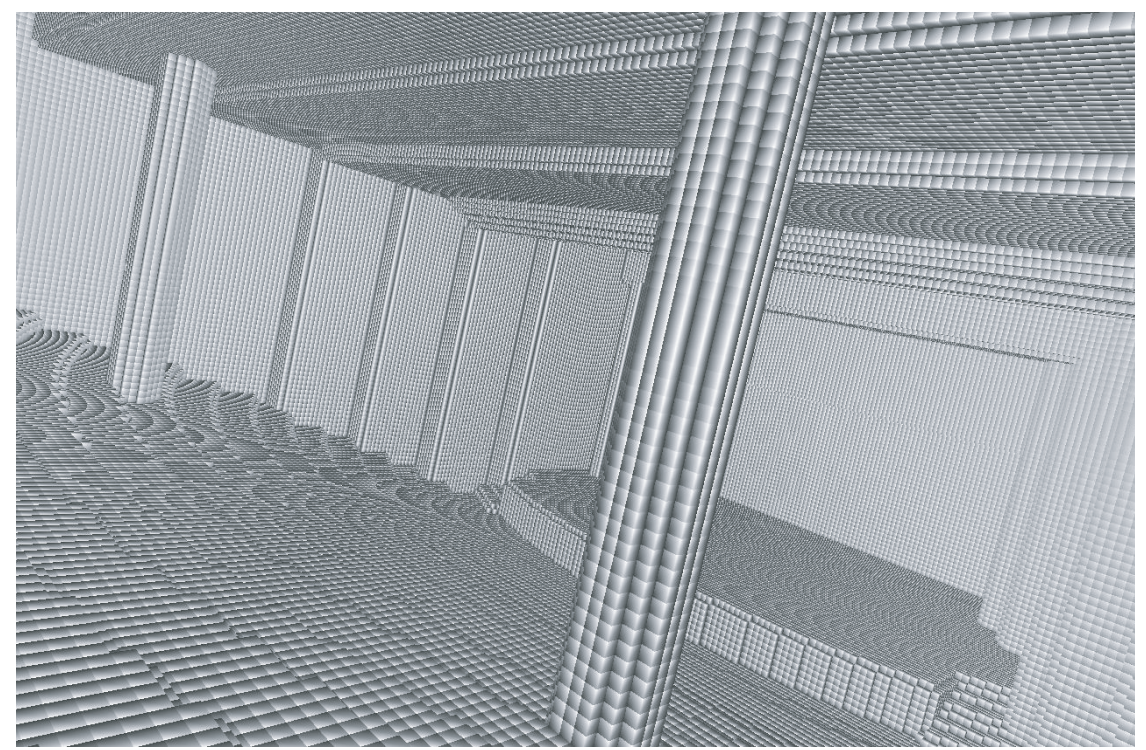

Figure B.6. Mesh of the auditorium (the cell size for this picture is smaller than the used in the simulation for clarity).

window. Buttons for loading the 3-D file defining the room, for generating the mesh and for executing the simulation can be observed. In order to add more functions, this software has the option to save a file containing a 3-D model of the mesh which has been created. This file can be loaded with any $3-\mathrm{D}$ CAD software viewer in order to verify that the created mesh is in accordance with the original CAD file.

Finally, there are a set of buttons to export the simulation results. It is possible to export the impulse response, which has been obtained between the defined source position and a set of listening positions. In this way, this software stores the pressure values for every defined point for each time step to create the room impulse response between the source and such points. Furthermore, the program can export pressure maps at different planes, perpendicular to the axis. In this GUI version, up to four planes can be defined. Such exportation can be done to ASCII format, to MATLAB ${ }^{\circledR}$ compatible files or to $\operatorname{VTK}^{\circledR}$ files.

The software also allows to modify and visualize the configuration pa- 
rameters related to the DWM simulation. It is also possible to define certain characteristics such as the bandwidth simulation limit or the oversampling applicable, which could be applied to minimize the inherent dispersion of DWM. Before starting the simulation or even before creating the mesh, it could be seen the mesh size for a given selected cell size. In this way, one can know a priori if the mesh is too large for the memory and power of the computer being used. In this sense, a window reports the available memory resources and the memory needed in the simulation.

Once the meshing is done, the program shows the number of active cells among those that comprise the mesh shoebox. Also, it offers an efficiency percentage of the memory use, i.e. the ratio between the room volume and the shoebox volume that contains the room. Additionally, one could check the number of cells that are located in the boundaries and, therefore, they will be treated in a special manner.

There are other areas in the window to configure both source positions and its excitation signals. If a delta function $\delta(n)$ is selected, its bandwidth will be half the sampling frequency and thus, there will be dispersion. However, to obtain with the selected bandwidth, the Gaussian or sinc signal option must be clicked; both have the $-6 \mathrm{~dB}$ bandwidth selected in the respective box. In addition, a wavelet signal can be used, with an octave bandwidth, centered in the mentioned frequency. This signal can be of interest when a room is studied in a given single octave. Moreover, it is possible to employ a user-defined excitation function via a script code. Finally, while the software is running, it allows iterative showing of a snapshot of a pressure map using a pseudocolor scale.

\section{B.3 Parallelization process}

The huge amount of computer power necessary to simulate large acoustical spaces limits the DWM use in personal computers (PC). However, computer power in PCs continues to grow following Moore's law [Moore, 1965]. Dual core microprocessors are typical today, quad core processors have just been introduced in the market and multiple core processors are in the sort term plans of CPU manufacturers. So in the coming years, personal computers will be able to manage acoustical simulations using DWM up to reasonable frequencies and for large rooms without problems. 


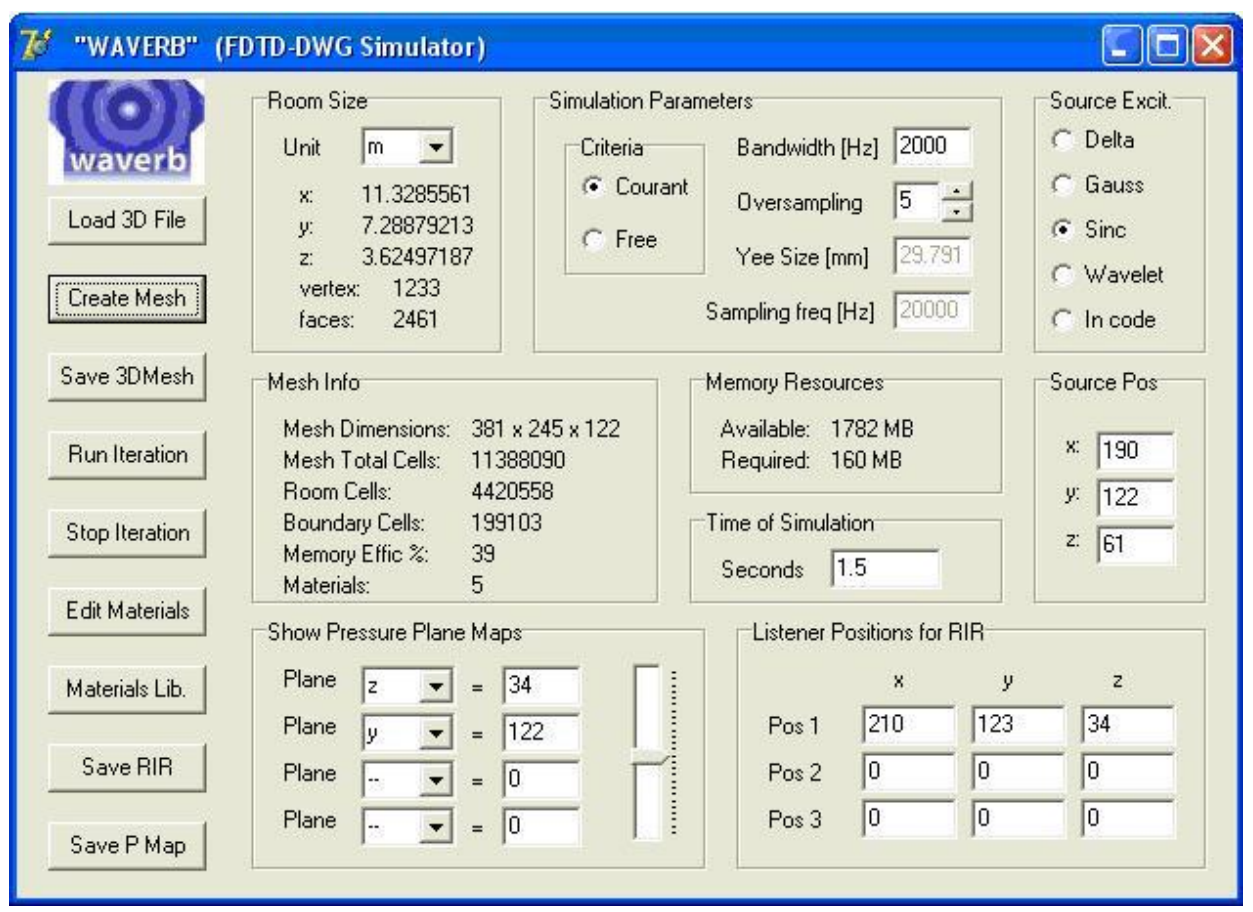

Figure B.7. Graphic User Interface for Waverb software.

However, at the moment, when a big enclosure is to be simulated up to a reasonable frequency using DWM, the numerical power and also the memory capacity of a PC is not enough. A simple but effective solution could be to divide the whole mesh into smaller volumes and computing them simultaneous. In these cases, parallel computing is one interesting solution.

The DWM algorithm (see Sec. 4.3) is easy to parallelize, only dividing the mesh into as many smaller blocks as processors are available. Each processor computes the DWM recursion only for the cells of the block.

The division into blocks can be carried out by splitting the mesh in slices on one axis, or into two or three. Depending on the number of processors or the parallel architecture used, one can be more interesting than the other. 


\section{B.3.1 Parallel architectures}

The classification of the parallel architectures in computer science may be very complex [Roosta, 2000]. In the following, the simplest classification, oriented to affordable computers or superb computers is presented. The aim is to understand which alternatives could be used to split the problem of a DWM recursion.

- Shared Memory Model: A set of processors (or cores) shares the same main system memory. Thus, all have access to the same data and the problem division is straightforward, since there is no communication between them, except from the synchronization of the different threads.

- Cluster of Computers: This model uses different computers connected to a high speed net. It is usual to employ low cost computers. In certain steps of the algorithm, when communication between them is necessary, a message parking system is employed. The most popular ones are the MPI [Snir et al., 1995] and the PVM [Geist et al., 1994]. As a drawback, this architecture presents two side effects: on the one hand, the software complexity arises because of all the communication procedures and, on the other hand, the algorithm is slowed down, since a great amount of time is wasted in each communication iteration. In the DWM case, in each temporal iteration, processors must communicate the value of the contact cells, since DWM recursion has dependency on adjacent cells.

- Supercomputers: This is the most complex case, in which very sophisticated architectures, vectorial processors, shared and not shared memory, ..., may coexist. The supercomputer power may simulate large models at high frequencies and at very high speed. In any case, the arise in the capacity of the current PC and the use of the previous approaches are still on the run.

\section{B.3.2 Hardware and software used in the experiments}

In the implementation of the DWM simulation software, special care has been taken to profit of the complex math instructions present in modern CISC CPU (x86 architecture). A C/C ++ compiler using advanced SS3 and x64 instructions has been employed. Some critical 
parts of the code have been implemented directly in the assembler for maximum performance. The algorithm has been implemented for parallel use inside the same PC (shared memory), so it can split the recursion between the different $\mathrm{CPU}$ cores present in the computer. The mesh can be split in one of the long axes, or even two or three. It has been tested for 8 cores, using a bi-processor motherboard containing two Quad-Core Intel $^{\circledR}$ Xeon ${ }^{\circledR} 5300$ processors, but it has been prepared to use more cores when available. An improved version of the software that can split the problem into a cluster of PCs using MPI is being developed.

\section{B.4 Computational cost and memory requirements}

Both, memory requirements and computational cost of the DWM recursion are related to the size of the cell. The Courant formula gives the size of the cell according to the sampling frequency, see Eq. 4.35. However, just the accomplishment of the Nyquist criteria $\left(f_{s}=2 f_{\max }\right)$ is not enough. The frequency-dependent dispersion, that it is inherent to the DWM method must be taken into account. According to the previous texts of this thesis, a solution is based on using a $2 \times$ oversampling, with a $f_{s}=4 f_{\max }$. This is computationally costly, but it allows a visualization on-line of the simulation. In this work, this technique has been chosen, in order to analyze the suitability of DWM in computational extreme conditions. In accordance with that, a table has been composed, that relates the number of cells per cubic meter and the maximum simulation frequency. This table helps to easily calculate the number of effective cells that an enclosure needs, for a given volume.

\begin{tabular}{|c|c|c|c|c|c|c|c|}
\hline Freq. (Hz) & 250 & 500 & 1000 & 2000 & 4000 & 8000 & 16000 \\
\hline Cells $/ \mathrm{m}^{3}$ & 5 & 39 & 313 & $2.5 \cdot 10^{3}$ & $2 \cdot 10^{4}$ & $1.6 \cdot 10^{5}$ & $1.3 \cdot 10^{6}$ \\
\hline
\end{tabular}

Table B.1. Number of cells per cubic meter as a function of the maximum frequency considered

However, as described in Sec. B.1, for efficiency in memory addressing, the size of the mesh would be the size of the minimum boundary box. According Eq. 4.37, the pressure at present time step, and the pressure at 
the two times steps before needs to be stored. Assuming the use of singleprecision floating-point arithmetic for pressure (4 bytes) and adding the matrix of labels per cell, the total memory in bytes needed is

$$
\text { Memory }=\text { Cells } \times(4 \times 3+1)
$$





\section{Bibliography}

M. Abramowitz and I. A. Stegun. Handbook of Mathematical Functions. Dover, 1970.

H. Ahlberg, E. Nielson, and J. Walsh. Theory of Splines and Their Applications. Academic Press, London, England, 1967.

W. Ahnert, M. Bansal, and S. Feistel. Large scale FEM analysis of a studio room by. In Proc. Audio Eng. Soc. Convention, pages Paris, France, 2006.

J. B. Allen and D. A. Berkley. Image method for efficiently simulating small-room acoustics. J. Acoust. Soc. Am., 65(4):943-950, 1979.

K. Attenborough. Ground parameter information for propagation modeling. J. Acoust. Soc. Am., 92(1):418-427, 1992.

M. R. Bai. Study of acoustic resonance in enclosures using eigenanalysis based on boundary element methods. J. Acoust. Soc. Am., 91:2529-2538, 1992.

C. A. Balanis. Advanced Engineering Electromagnetism. J. Wiley \& Sons, 1989.

C. A. Balanis. Antenna theory. John Wiley \& Sons, 1997. 
A. Bayliss, M. Gunzburger, and E. Turkel. Boundary conditions for the numerical solution of elliptic equations in exterior regions. SIAM J. Applied Mathematics, 42:430-451, 1982.

A. Bayliss and E. Turkel. Radiation boundary conditions for wave-like equations. Comm. Pure Applied Mathematics, 23:707-725, 1980.

M. J. Beeson and D. Murphy. Roomweaver: A digital waveguide mesh based room acoustics research tool. In Proc. of the 7th International Conference on Digital Audio Effects (DAFX-04), Naples, Italy, 2004.

R. Begault. 3-D Sound for Virtual Reality and Multimedia. Academic Press Professional, 1994.

J. H. Beggs. The linear bicharacteristic scheme for electromagnetics. Technical Report NASA-TM-2001-210861, NASA, Langley Research Center, Hampton, VA, 2001.

J. H. Beggs, R. J. Luebbers, K. Yee, and K. Kunz. Finite-difference timedomain implementation of surface impedance boundary conditions. IEEE Trans. on Antennas and Propagation, 40:1223-1230, Oct. 1992.

L. L. Beranek. Acoustic impedance of commercial materials and the perfomance of rectangular rooms with one treated surface. J. Acoust. Soc. Am., 12:14-23, 1940.

L. L. Beranek. Acoustics. McGraw-Hill, 1954.

L. L. Beranek. Music, acoustics and architecture. J. Wiley, New York/London, 1962.

J. P. Berenger. A perfectly matched layer for the absorption of electromagnetic waves. J. Computat. Physics, 114:185-200, Oct. 1994.

P. G. Bergmann. The wave equation in a medium with variable index of refraction. J. Acoust. Soc. Am., 17:329-333, 1946.

S. D. Bilbao. Wave and scattering methods for the numerical integration of partial differential equations. PhD thesis, Electrical Engineering Department, Stanford University, 2001. 
S. D. Bilbao. Wave and scattering methods for the numerical integration of partial differential equations. John Wiley \& Sons, New York, USA, May 2004 .

D. M. Boore. Finite-difference methods for seismic wave propagation in heterogeneous materials, volume 11. Academic Press, New York, 1972.

J. Borish. Extension of the image model to arbitrary polyhedra. J. Acoust. Soc. Am., 75(6):1827-1836, 1984.

D. Botteldooren. Acoustical finite-difference time-domain simulation in a quasi-cartesian grid. J. Acoust. Soc. Am., 95:2313-2319, 1994.

D. Botteldooren. Finite-difference time-domain simulation of low-frequency room acoustic problems. J. Acoust. Soc. Am., 98(6):3302-3308, Dec. 1995.

D. Botteldooren. Time-domain simulation of the influence of close barriers on sound propagation to the environment. J. Acoust. Soc. Am., 101: 1278-1285, 1997.

P. A. Butov. Reflection of a spherical wave from an impedance boundary. Sov. Phys. Acoust., 27:191-193, 1981.

G. Campos. Three-dimensional waveguide mesh modelling for room acoustic simulation: MPhil/DPhil transfer report. Technical report, Dept. Electronics, University of York, 1999.

G. Campos and D. M. Howard. On the computational efficiency of different waveguide mesh topologies for room acoustic simulation. IEEE Trans. on Speech and Audio Processing, 13(5):1063-1072, Sept 2005.

M. F. Cátedra, J. Pérez, F. S. de Adana, and O. Gutiérrez. Efficient raytracing techniques for three-dimensional analyses of propagation in mobile communications: Application to picocell ands microcell scenarios. IEEE Antennas and Propagation Magazine, 40(2):15-28, 1998.

H. L. Châtelier. Experimental and theoretical research on chemical equilibrium. Ann. Mines Carburants, 8(13):157-380, 1888.

G. Chen and J. Zhou. Boundary Element Methods. Academic Press, 1992. 
C. Christopoulos. The Transmission-Line Modelling Method. Institute of Electrical and Electronics Engineers, 1995.

C. Coleman. A ray tracing formulation and its application to some problems in over-the-horizon radar. Radio Science, 33:1187-1197, 1998.

R. Courant. Variational methods for the solution of problems of equilibrium and vibrations. Bull. Am. Math. Soc., 49:1-23, 1943.

R. Courant, K. Friedrichs, and H. Lewy. Über die partiellen differenzengleichungen der mathematischen physik. Mathematische Annalen, 100(1): $32-47,1928$.

T. J. Cox, B.-I. L. Dalenbäck, P. D'Antonio, J. J. Embrechts, J. Y. Jeon, E. Mommertz, and M. Vorländer. A tutorial on scattering and diffusion coefficients for room acoustic surfaces. Acta Acust. united Ac., 92:1-15, 2006.

B.-I. Dalenbäck, M. Kleiner, and P. Svensson. Audibility of changes in geometric shape, source directivity, and absorptive treatment - experiments in auralization. J. Audio Eng. Soc., 41:905-913, 1993.

B.-I. L. Dalenbäck. Room acoustic prediction based on a unified treatment of diffuse and specular reflection. J. Acoust. Soc. Am., 100(2):899-909, 1996.

G. de Poli and D. Rocchesso. Tutorial article: physically based sound modelling. Organized sound, 3(1):61-76, 1998.

G. de Sanctis, A. Sarti, and S. Tubaro. Automatic synthesis strategies for object-based dynamical physical models in musical acoustics. In in Proc. of the 5th International Conference on Digital Audio Effects (DAFx-03), Sept 2003.

M. E. Delany and E. N. Bazley. Acoustical properties of fibrous absorbent materials. Appl. Acoust., 3:105-116, 1970.

J. Dominguez. Boundary Elements in Dynamics. Elsevier Applied Science, 1993.

H. Dong, A. M. Kaynia, C. Madshus, and J. M. Hovem. Sound propagation over layered poro-elastic ground using a finite-difference model. J. Acoust. Soc. Am., 108:494-502, 2000. 
S. A. V. Duyne. Digital filter applications to modeling wave propagation in springs, strings, membranes and acoustical space. PhD thesis, Department of Music, Stanford University, US, 2007.

S. A. V. Duyne and J. O. Smith. Physical modeling with the 2-D digital waveguide mesh. In Proc. of the 1993 International Computer Music Conference, 1993.

S. A. V. Duyne and J. O. Smith. The 3-D tetrahedral digital waveguide mesh. In Proc. of the IEEE Workshop on Applications of Signal Processing of Audio and Acoustics, New Platz, NY, 1995.

S. Elmasri, X. Pelorson, P. Saguet, and P. Badin. The use of the transmisson line matrix in acoustics and in speech. International Journal of Numerical Modelling, 11:133-151, 1998.

T. F. W. Embleton, J. E. Piercy, and N. Olson. Outdoor sound propagation over ground of finite impedance. J. Acoust. Soc. Am., 59:267-277, 1976.

J. J. Embrechts. Broad spectrum diffusion model for room acoustics raytracing algorithms. J. Acoust. Soc. Am., 107(4):2068-2081, 2000.

C. Erkut and M. Karjalainen. Finite difference method vs. digital waveguide method in string instrument modeling and synthesis. In Proc. ISMA 2003, Mexico City, Mexico, 2002a.

C. Erkut and M. Karjalainen. Virtual strings based on a 1-D FDTD waveguide model: Stability, losses, and traveling waves. In Proc. AES 22th Int. Conf. on Virtual, Synthetic, and Entertainment Audio, Espoo, Finland, 2002b.

J. Escolano, S. Bleda, B. Pueo, and J. J. López. Wave field synthesis 3D simulator based on finite-difference time-domain method. In Proc. 116th Audio Eng. Soc. Convention, Berlin, Germany, 2004.

J. Escolano and F. Jacobsen. A note on the physical interpretation of frequency dependent boundary conditions in a digital waveguide mesh. Acta Acust. united Ac., 93(3):398-402, May/Jun 2007.

J. Escolano, F. Jacobsen, and J. J. López. An efficient realization of frequency dependent boundary conditions in a finite-difference time-domain model. J. Sound Vibration, 316:234-247, 2008a. 
J. Escolano and J. J. López. On the adaptation of the linear bicharacteristic scheme to block-based physical modeling for digital sound synthesis of string instruments. In 31th International Conference on Acoustics, Speech, and Signal Processing (ICASSPŠO6), Toulouse, France, May 2006a.

J. Escolano and J. J. López. Upwind leapfrog schemes in physical models with mixed modeling strategies. In Proc. 120th Audio Eng. Soc. Convention, Paris, France, 2006b.

J. Escolano and J. J. López. Broadband directive sources modeling for acoustic discrete-time domain methods. J. Acoust. Soc. Am., 123(5): 3798, 2008. Abstract.

J. Escolano, J. J. López, and B. Pueo. Directive sources in acoustic discretetime domain simulations based on directivity diagrams. J. Acoust. Soc. Am., 121(6):EL256-EL262, Jun 2007.

J. Escolano and B. Pueo. Acoustic equations in the presence of rigid porous materials adapted to the finite-difference time-domain method. J. of Comput. Acoustics, 15(2):255-269, June 2007.

J. Escolano, B. Pueo, J. J. López, and M. Cobos. Empirical evaluation of the frequency-dependent boundary conditions in a Digital Waveguide Mesh. In Proc. 124th Audio Eng. Soc. Convention, Amsterdam, Holland, May 2008b.

F. Everest. The Master Handbook of Acoustics. Blue Ridge Summit, Pa, 1994.

F. Fahy. Foundations of Engineering Acoustics. Academic Press, 2001.

A. Farina. An example of adding spatial impression to recorded music: signal convolution with binaural impulse responses. In Proc. of the International Conference Acoustics and Recovery of Spaces for Music, Ferrrara, Italy, 1993.

A. Farina. RAMSETE - a new pyramid tracer for medium and large scale acoustic problems. In Proc. Euro-Noise 95, March 1995.

A. Fettweis. Wave digital filters: Theory and practice. Proceedings of the IEEE, 74(2):270-327, 1986. 
A. Fettweis and G. Nitsche. Numerical integration of partial differential equations using principles of multidimensional wave digital filters. Journal of VLSI Signal Processing, 3(1/2):7-27, 1991.

J. L. Flanagan. Analog measurements of sound radiation from the mouth. J. Acoust. Soc. Am., 32(12):1613-1620, 1960.

H. Fleischer. Die Pauke: Mechanismer Schwinger und akustiche Strahler. Univ. der Bundeswehr, Munich, Germany, 1998.

N. H. Fletcher and T. D. Rossing. The physics of musical instruments. Springer Verlag, 1991.

F. Fontana. Physics-based models fot the acoustic representation of space on virtual environments. PhD thesis, Dept. of Computer Science, University of Verona, Italy, 2003.

F. Fontana and D. Rocchesso. A new formulation of the 2D-waveguide mesh for percussion instruments. In Proc. of the XI Colloquium in Musical Informatics, 1995.

F. Fontana and D. Rocchesso. Physical modeling of membranes for percussion instruments. Acta Acust. united Ac., 83:529-542, 1998.

F. Fontana and D. Rocchesso. Online correction of dispersion error in 2D waveguide meshes. In Proc. Int. Computer Music Conf.,(ICMC'00), 2000.

F. Fontana and D. Rocchesso. Signal-theoretic characterization of waveguide mesh geometries for models of two-dimensional wave propagation in elastic media. IEEE Trans. on Speech and Audio Processing, 9:152-161, 2001.

B. Friedlander and B. Porat. The modified Yule-Walker method of ARMA spectral estimation. IEEE Trans. on Aerospace Electronic Systems, 20 (2):158-173, 1984.

K. Y. Fung and H. Ju. Time-domain impedance boundary conditions for computational acoustics and aeroacoustic. J. Computat.l Fluid Dynamics, 18:503-511, 2004.

T. Funkhouser, I. Carlbom, G. Elko, G. Pingali, M. Sondhi, and J. West. A beam tracing approach to acoustic modeling for interactive environments. 
In In Proceedings of the 25th annual conference on Computer graphics and interactive techniques, pages 21-32, New York, NY, USA, 1998. ACM Press.

T. Funkhouser, N. Tsingos, I. Carlbom, G. Elko, M. Sondhi, J. West, G. Pingali, P. Min, and A.Ngan. A beam tracing method for interactive architectural acoustics. J. Acoust. Soc. Am., 115(2):739-756, 2004.

A. J. Gandomi and H. Takenaka. Efficient FDTD algorithm for plane-wave simulation for vertically heterogeneous attenuative media. Geophysics, 72(4):H43-H53, July/August 2007.

A. Geist, A. Beguelin, J. Dongarra, W. Jiang, B. Manchek, and V. Sunderam. PVM: Parallel Virtual Machine-A User's Guide and Tutorial for Network Parallel Computing. MIT Press, 1994.

A. S. Glassner. An introduction to Ray Tracing. Academic Press, 1989.

R. W. Graves. Simulating seismic wave propagation in 3-D elastic media using staggered-grid finite differences. Bull. Seismol. Soc. Am., 86:1091106, 1996.

H. Hacıhabiboğlu, B. Günel, and A. M. Kondoz. Source directivity simulation in digital waveguide mesh based room acoustics models. In Proc. of the AES 30th International Conference on Intelligent Audio Environments, Saariselkä, Finland, 2007.

H. Hacıhabiboğlu, B. Günel, and A. M. Kondoz. On the accuracy of firstorder numerical derivatives in multidimensional digital waveguide mesh topologies. IEEE Signal Processing Letters, 15(1):9-12, 2008a.

H. Hacıhabiboğlu, B. Günel, and A. M. Kondoz. Time-domain simulation of directive sources in 3D digital waveguide mesh-based acoustical models. IEEE Trans. Audio, Speech, and Language Processing, 16(5):934-946, $2008 \mathrm{~b}$.

J. H. Halton. A retrospective and prospective of the Monte Carlo method. SIAM Review, 12(1):1-63, Jan 1970.

K. Heutschi, M. Horvath, and J. Hofmann. Simulation of ground impedance in finite difference time domain calculations of outdoor sound propagation. Acta Acust. united Ac., 91:35-40, 2005. 
R. Higdon. Absorbing boundary conditions for difference aproximation to the multidimensional wave equation. Mathematics on Computations, 47: 437-459, 1986.

R. Higdon. Numerical absorbing boundary conditions for the wave equation. Mathematics on Computations, 49:65-90, 1987.

M. Hodgson. Case history: Factory noise prediction using ray tracing experimental validation and the effectiveness of noise control measures. Noise Control Engineering Journal, 33(3):97-104, 1989.

M. Hodgson and E.-M. Nosal. Experimental evaluation of radiosity for room sound-field prediction. J. Acoust. Soc. Am., 120:808-819, 1996.

J. Huopaniemi, M. Karjalainen, V. Välimäki, and T. Huotilainen. Virtual instruments in virtual rooms-a real-time binaural room simulation environment for physical models of musical instruments. In Proc. of Int. Comp. Music Conference (ICMC'94), Aarhus, Denmark, 1994.

J. Huopaniemi, K. Kettunen, and J. Rahkonen. Measurement and modelling techniques for directional sound radiation from the mouth. In Proc. IEEE Workshop Appl. Signal Process. Audio Acoust. (WASPAAS̆99), pages 183-186, New Paltz, NY, USA, 1999.

J. Huopaniemi, L. Savioja, and M. Karjalainen. Modeling of reflections and air absorption in acoustical spaces: a digital filter design approach. In IEEE Workshop on Applications of Signal Processing to Audio and Acoustics (WASPAA'97), New Platz, NY, USA, Oct. 1997.

F. Ihlenburg. Finite Element Analysis of Acoustic Scattering. SpringerVerlag, New York, USA, 1998.

F. Jacobsen. A note on finite difference estimation of acoustic particle velocity. J. Sound Vibration, 256(5):849-859, 2002.

F. Jacobsen. Sound intensity and its measurement and applications. Technical Report 31262, Acoustic Technology, ØrstedŢDTU, Technical University of Denmark, 2006.

F. Jacobsen. Handbook of Acoustics, chapter Sound intensity, pages 10531075. Springer, 2007. 
H. Jeong, I. Drumm, B. Horne, and Y. W. Lam. The modelling of frequency dependent boundary conditions on FDTD simulation of concert hall acoustics. In Proc.19th International congress on acoustics (ICA'07), Madrid, Spain, 2007.

Z. Ji, B.-H. Li, H.-X. Wang, H.-Y. Chen, and T. K. Sarkar. Efficient raytracing methods for propagation prediction for indoor wireless communications. IEEE Antennas and Propagation Magazine, 43(2):41-49, 2001.

J. Jin. The Finite Element Method in Electromagnetics. Wiley-IEEE Press, 2002.

P. Johns. On the relationship between TLM and finite-difference methods for Maxwell's equations. IEEE Trans. Microwave Theory Tech., 35(1), 1987.

C. Jordan. Calculus of finite differences. Chelsea Publishing Company, New York, NY, 1950.

B. Kapralos. The sonel mapping acoustical modeling method. PhD thesis, York University, Toronto, Canada, 2006.

M. Karjalainen. Mixed physical modeling: DWG + FDTD + WDF. In in Proc. IEEE Int. Conf. Acoustics, Speech, and Signal Processing, New Platz, NY, 2003.

M. Karjalainen. Discrete-time modeling and synthesis of musical instruments. In Joint Baltic-Nordic Acoustics Meeting 2004, Mariehamn, Åland, June 2004.

M. Karjalainen. Efficient realization of wave digital components for physical modeling and sound synthesis. IEEE Trans. Audio, Speech and Language Processing, 16(5):947-956, 2008.

M. Karjalainen and C. Erkut. Digital waveguides vs. finite difference schemes: Equivalence and mixed modeling. EURASIP J. Appl. Signal Proc., 2004(7):978-989, 2004.

M. Karjalainen, C. Erkut, and L. Savioja. Compilation of unified physical models for efficient sound synthesis. In in Proc. IEEE Int. Conf. Acoustics, Speech, and Signal Processing, 2003. 
T. K. Katsibas and C. S. Antonopoulos. A general form of perfectly matched layers for three-dimensional problems of acoustic scattering in lossless and lossy fluid media. IEEE Trans. on Ultrasonics, Ferroelectrics, and Frequency Control, 51(8):964-972, 2004.

T. K. Katsibas and C. S. Antonopoulos. Development and evaluation of a complete 3D FDTD computational algorithm for the numerical approximation of guided acoustic wave propagation in lossy media. Acta Acust. united Ac., 93(6):861-869, 2007.

A. Kelloniemi. Improved adjustable boudnary condition for the 2-D digital waveguide mesh. In Proc. of the 5th Int. Conf. on Digital Audio Effects (DAFx'05), Madrid, Spain, Sept. 2005a.

A. Kelloniemi. Improved adjustable boundary condition for the 3-D digital waveguide mesh. In Proceedings of the 2005 IEEE Workshop on Applications of Signal Processing to Audio and Acoustics (WASPAA 2005), pages 191-194, Oct 2005b.

A. Kelloniemi. Frequency-dependent boundary condition for the 3-D digital waveguide mesh. In Proceedings of the 9th International Conference on Digital Audio Effects (DAFx-06), pages 161-164, Montreal, Canada, 2006.

A. Kelloniemi, D. T. Murphy, L. Savioja, and V. Välimäki. Boundary conditions in a multi-dimensional digital waveguide mesh. In Proceedings of the 29th IEEE International Conference on Acoustics, Speech, and Signal Processing (ICASSP 2004), volume 4, pages 25-28, 2004.

A. Kelloniemi, L. Savioja, and V. Välimäki. Spatial filter based absorbing boundary for the 2-D digital waveguide mesh. IEEE Signal Processing Letters, 12(2):126-129, 2005.

K. R. Kelly, R. W. Ward, S. Treitel, and R. M. Alford. Synthetic seismograms: a finite difference approach. Geophysics, 41:2-27, 1976.

C. Kim. Multidimensional Upwind Leapfrog Schemes and Their Applications. PhD thesis, University of Michigan, 1997.

S. Kirkup. The boundary element method in acoustics. Integrated Sound Software, 1998. 
M. Kleiner, P. Svensson, and B.-I. Dalenbäck. Auralization: Experiments in acoustical CAD. In Proc. of the 89th Audio Eng. Soc. Convention, Los Angeles, USA, 1990.

K. Kowalczyk and M. van Walstijn. Formulation of a locally reacting wall in finite difference modelling of acoustic spaces. In Proc. of the International Symposium on Room Acoustics, Sevilla, Spain, Sept 2007.

K. Kowalczyk and M. van Walstijn. Virtual room acoustics using finite difference methods. how to model and analyse frequency-dependent boundaries? In Proc. Int. Symp. Communications, Control and Signal Processing (ISCCSP'08), pages 1-6, Malta, March 2008.

H. Krauss and R. Rabenstein. Application of multidimensional wave digital filters to boundary value problems. IEEE Signal Processing Letters, 2(7): 361-382, 1995.

H. Krauss, R. Rabenstein, and M. Gerken. Simulation of wave propagation by multidimensional wave digital filters. Simulation Practice and Theory, 4:361-382, 1996.

J. Kristek and P. Moczo. On the accuracy of the finite-difference schemes: The 1-D elastic problem. Seismological Soc. Am., 96:2398-2414, 2006.

A. Krokstad, S. Strom, and S. Sorsdal. Calculating the acoustical room response by the use of a ray tracing technique. J. Sound Vibration, 8(1): $118-125,1968$.

W. Kropp and P. U. Svensson. Application of the time domain formulation of the method of equivalent sources to radiation and scattering problems. Acustica, 91:528-543, 1995.

M. Krumpholz, C. Huber, and P. Russer. A field-theoretical comparison of FDTD and TLM. IEEE Trans. Microwave Theory Tech., 43(8):19351950, 1995.

A. Kulowski. Algorithmic representation of the ray tracing technique. Appl. Acoust., 18(6):449-469, 1985.

A. Kuntz and R. Rabenstein. A 3D acoustic simulation program with graphical frontend for scene input. In Proc. 120th Audio Eng. Soc. Convention, Paris, France, 2006. 
A. Kuntz and R. Rabenstein. Room acoustic analysis: Complementing 2D measurements by 3D simulations. In 3rd International Symposium on Communications, Control and Signal Processing (ISCCSP'08), St. Julian, Malta, 2008.

K. S. Kunz and R. J. Luebbers. The Finite Difference Time Domain Method for Electromagnetics. CRC Press, 1993.

H. Kuttruff. Room Acoustics. Taylor \& Francis, 4th edition, 2000.

T. I. Laakso, V. Välimäki, M. Karjalainen, and U. K. Laine. Splitting the unit delay - tools for fractional delay filter design. IEEE Signal Processing Magazine, 13(1):30-60, Jan 1996.

J. Laird, P. Masri, and N. Canagarajah. Modelling diffusion at the boundary of a digital waveguide mesh. In Proc. of the International Computer Music Conference (ICMC'99), Hong Kong, Oct 1999.

Y. W. Lam. A comparison of three diffuse reflection modeling methods used in room acoustics computer models. J. Acoust. Soc. Am., 100(4): 2181-2192, 1996.

K. Lee and J. O. Smith. Implementation of a highly diffusing 2-D digital waveguide mesh with a quadratic residue diffuser. In in Proc. of the International Computer Music Conference (ICMC'04), Antalaya, Turkey, Sept. 2005.

H. Lehnert. Systematic errors of the ray-tracing algorithm. Appl. Acoust., 38:207-221, 1993.

S. G. Lipson, H. Lipson, and D. S. Tannhauser. Optical Physics. Cambridge University Press, 1995.

L. Liu and D. Albert. Acoustic pulse propagation near a right-angle wall. J. Acoust. Soc. Am., 119(4):2073-83, 2006.

Q. H. Liu and J. Tao. The perfectly matched layer for acoustic waves in absorptive media. J. Acoust. Soc. Am., 102:2072-2082, 1997.

T. Lokki and L. Savioja. Room acoustic modeling. In 124th Audio Eng. Soc. Convention, Amsterdam, Holland, 2008. 
J. J. López, J. Escolano, and B. Pueo. On the implementation of a room acoustics modeling software using finite differences time domain methods. In Proc. of the 122th Audio Eng. Soc. Convention, pages Wien, Austria, 2007a.

J. J. López, J. Escolano, and B. Pueo. Simulation of complex and large rooms using a digital waveguide mesh. In Proc of 123th Audio Eng. Soc. Convention, New York, USA, 2007b.

J. Lovetri, D. Mardare, and G. Soulodre. Modeling of the seat dip effect using the finite-difference time-domain method. J. Acoust. Soc. Am., 100: 2204-2212, 1996.

J. G. Maloney and K. E. Cummings. Adaptation of FDTD techniques to acoustic modeling. In 11th Annual Review of Progress in Applied Computational Electromagnetics, Monterey, Canada, Mar. 1995.

J. G. Maloney and G. S. Smith. The use of surface impedance concepts in the finite-difference time-domain method. IEEE Trans. Antennas Propagat., 40:49-56, Jan. 1992.

J. D. Markel and A. H. Gray. Linear Prediction of Speech. Springer Verlag, New York, USA, 1976.

J. W. McJown and R. L. Hamilton. Ray-tracing as a design tool for radio networks. IEEE Network Magazine, 5(6):27-30, 1991.

S. Miklavcic and J. Ericsson. Practical implementation of the 3D tetrahedral TLM method and visualization of room acoustics. In Int. Conference on Digital Audio Effects (DAFx $\check{S} 04$ ), pages 262-267, Naples, Italy, 2004.

G. E. Moore. Cramming more components onto integrated circuits. Electronics Magazine, 38(8), May 1965.

J. N. Mourjopoulos, E. D.Kyriakis-Bitzaros, and C. E.Goutis. Theory and real-time implementation of time-varying digital audio filters. J. Audio Eng. Soc., 38(7/8):523-536, 1990.

G. Mur. Absorbing boundary conditions for the finitte-difference approximation of the time-domain electromagnetic field equations. IEEE Trans. Electromagn, Compat., 23:377-382, 1981. 
D. T. Murphy. Digital waveguide mesh topologies in room acoustics modelling. PhD thesis, Dept. Electronics, University of York, UK, 2000.

D. T. Murphy and M. Beeson. The KW-boundary hybrid digital waveguide mesh for room acoustics applications. IEEE Trans. on Speach and Audio Processing, 15(2):552-564, March 2007.

D. T. Murphy, M. Beeson, S. Shelley, A. Southern, and A. Moore. RenderAIR - room acoustics simulation using a hybrid digital waveguide mesh. In Proc. 124th Audio Eng. Soc. Convention, Amsterdam, Holland, 2008a.

D. T. Murphy, A. Kelloniemi, J. Mullen, and S. Shelley. Acoustic modeling using the digital waveguide mesh. IEEE Signal Processing Magazine, 24 (2):55-66, March 2007.

D. T. Murphy and J. Mullen. Digital waveguide mesh modelling of room acoustics: Improved anechoic boundaries. In In Proc. Sixth Conf. on Digital Audio Effects (DAFx-02), pages 163-168, Hamburg, Germany, 2002.

D. T. Murphy, S. Shelley, and A. Southern. Diffusing boundaries in the digital waveguide mesh and their effect on reverberation time. In Proc. 3rd Int. Symposium on Communications, Control and Signal Processing (ISCCSP 2008), St. Julians, Malta, 2008b.

G. Naylor. ODEON - another hybrid room acoustical model. Appl. Acoust., 38:131-143, 1993.

B. Nguyen. Investigation of Three-Level Finite-Difference Time-Domain Methods for Multidimensional Acoustics and Electromagnetics. PhD thesis, University of Michigan, 1996.

E.-M. Nosal, M. Hodgson, and I. Ashdown. Investigation of the validity of radiosity for sound-field prediction in cubic rooms. J. Acoust. Soc. Am., 116:3505-3514, 2004.

M. Ochmann. The source simulation technique for acoustic radiation problems. Acustica, 81:512-527, 1995.

M. Ochmann. The full-field equations for acoustic radiation and scattering. J. Acoust. Soc. Am., 105(5):2574-2584, 1999. 
S. K. Olesen. Low frequency room simulation using finite difference equations. In Proc. 102th Audio Eng. Soc. Convention, Munich, Germany, Mar. 1997.

A. V. Oppenheim, R. W. Schafer, and J. R. Buck. Discrete-Time Signal Processing. Prentice Hall, 1999.

T. Osa, K. Murakamib, Y. Horinouchia, and D. Takahashia. Application of audience-seats characteristics to the sound field analysis for large enclosures. Appl. Acoust., 68(9):939-952, 2006.

V. Ostashev, D. Wilson, L. Liu, D. Aldridge, N. Symons, and D. Marlin. Equations for finite-difference time-domain simulation of sound propagation in moving inhomogeneous media and numerical implementation. $J$. Acoust. Soc. Am., 117(2):503-17, 2005.

V. E. Ostashev, S. L. Collier, D. K. Wilson, D. F. Aldridge, N. P. Symons, and D. Marlin. Padé approximation in time-domain boundary conditions of porous surfaces. J. Acoust. Soc. Am., 122:107-112, 2007.

F. Otondo and J. H. Rindel. The influence of the directivity of musical instruments in a room. Acta Acust. united Ac., 90:1178-1184, 2004.

Y. Özyörük and L. N. Long. A time-domain implementation of surface acoustic impedance condition with and without flow. J. Comput. Acoust., 5(3):277-196, 1997.

T. Parks and C. Burrus. Digital Filter Design. Ed. John Wiley \& Sons, 1987.

S. Petrausch. Block-Based Physical Modeling. PhD thesis, Chair of Multimedia Communications and Signal Processing, University of ErlangenNuremberg, Erlangen, Germany, 2007.

S. Petrausch, J. Escolano, and R. Rabenstein. A general approach to blockbased physical modeling with mixed modeling strategies for digital sound synthesis. In Proc. Int. Conf. on Acoustics, Speech and Signal Processing (ICASSP'05), volume 3, pages 21-24, 2005a.

S. Petrausch and R. Rabenstein. Interconnection of state space structures and wave digital filters. IEEE Trans. on Circuits and Systems II, 52(2): 90-93, Feb 2004. 
S. Petrausch and R. Rabenstein. Efficient 3-D simulation of wave propagation with the functional transformation method. In 18th Symposium of Simulation Technique, Erlangen, Germany, 2005a.

S. Petrausch and R. Rabenstein. Simulation of room acoustics via blockbased physical modeling with the functional transformation method. In IEEE Workshop on Applications of Signal Processing to Audio and Acoustics (WASPAA'05), pages 195-198, New Paltz, New York, 2005b.

S. Petrausch and R. Rabenstein. Wave field simulation with the functional transformation method. In iin IEEE Proc. Int. Conf. on Acoustics, Speech 65 Signal Processing (ICASSP'06), Toulouse, France, May 2006.

S. Petrausch and R. Rabenstein. Application of mixed modeling strategies for the simulation of the $2 \mathrm{~d}$ wave equation for arbitrary geometries. In 15th European Signal Processing Conference (EUSIPCO'07), pages 3-7, Poznan, Poland, Sep 2007.

S. Petrausch, S. Spors, and R. Rabenstein. Simulation and visualization of room compensation for wave field synthesis with the functional transformation method. In Proc. 119th Audio Eng. Soc. Convention, pages New York, USA, 2005b.

A. D. Pierce. Acoustics, An Introduction to Its Physical Principles and Applications. Acoustical Society of America, 1994.

A. D. Pierce. Handbook of Acoustics, chapter Basic linear acoustics, pages 25-112. Springer, 2007.

A. Pietrzyk and M. Kleiner. The application of the finite-element method to the prediction of soundfields of small rooms at low frequencies. In Proc. 102th Audio Eng. Soc. Convention, pages Munich, Germany, 1997.

A. D. Polyanin and V. F. Zaitsev. Handbook of Exact Solutions for Ordinary Differential Equations. CRC Press, Boca Raton, 2nd edition, 2003.

J. G. Proakis and D. G. Manolakis. Digital Signal Processing. Principles, algorithms and applications. Prentice Hall, 1998.

R. Rabenstein, S. Petrausch, A. Sarti, G. de Sanctis, C. Erkut, and M. Karjalainen. Block-based physical modeling for digital sound synthesis. IEEE Signal Processing Magazine, 24(2):42-54, March 2007. Special issue on sound synthesis. 
R. Rabenstein and T. Schetelig. A multidimensional wave digital filter algorithm for the 3-D simulation of room acoustics. In Proc. IEEE Image and Multidimensional Digital Signal Processing Workshop (IMDSP98), pages 255-258, Alpbach, Austria, Jul. 1998.

G. Ramos and J. López. Filter design method for loudspeaker equalization based on IIR parametric filters. J. Audio Eng. Soc., 54(12):1162-1178, Dec 2006.

C. R. Rao and S. K. Mitra. Generalized Inverse of Matrices and Its Applications. J. Wiley \& Son, New York, USA, 1971.

J. Redondo, R. Picó, B. Roig, and M. Avis. Time domain simulation of sound diffusers using finite-difference schemes. Acta Acust. united Ac., 93(4):611-622, 2007.

S. W. Rienstra. 1-D reflection at an impedance wall. J. Sound Vibration, 125(1):43-51, 1998.

D. D. Rife and J. Vanderkooy. Transfer-function measurements using maximum-length sequences. J. Audio Eng. Soc., 37(6):419-444, 1989.

J. Rindel. Computer simulation techniques for acoustical design of rooms. Acoustics Australia, 23:81-86, 1995.

D. Rochesso and J. O. Smith. Generalized digital waveguide networks. IEEE Trans. on Speech and Audio Processing, 11(3):242-254, 2003.

P. Roe. Linear bicharacteristic schemes without dissipation. SIAM J. Sci. Comput., 19(5):1405-1427, Sept 1998.

S. H. Roosta. Parallel processing and parallel algorithms: theory and computation. Springer, 2000.

D. A. Russell, J. P. Titlow, and Y. J. Bemmen. Acoustic monopoles, dipoles, and quadripoles: An experiment revisited. Am. J. Phys., 67(8):660-664, 1999.

S. Sakamoto, T. Seimiya, and H. Tachibana. Visualization of sound reflection and diffraction using finite difference time domain method. Acoust. Sci \& Tech., 23(1):34-39, 2002. 
S. Sakamoto, A. Ushiyama, and H. Nagatomo. Sound field simulation of a hall using FDTD method. Technical Report AA2005-41, Archit. Acoust. in Acoust. Soc. Jpn., 2005.

L. Savioja. Modeling Techniques for Virtual Acoustics. PhD thesis, Helsinki University of Technology, Telecommunications Software and Multimedia Laboratory, Espoo, Finland, 1999.

L. Savioja, J. Backman, A. Järvinen, and T. Takala. Waveguide mesh method for low-frequency simulation of room acoustic. In Proc. 15th Int. Congr. Acoust. (ICA'95), volume 2, pages 637-640, June 1995. Trondheim, Norway.

L. Savioja, J. Huopaniemi, T. Lokki, and R. Väänänen. Creating interactive virtual acoustic environments. J. Audio Eng. Soc., 47(9):675-705, 1999.

L. Savioja, A. Järvinen, K. Melkas, and K. Saarinen. Determination of the low frequency behaviour of an IEC listening room. In Proc. of the Nordic Acoustical Meeting, pages 55-58, Helsinki, Finland, 1996a.

L. Savioja, M. Karjalainen, and T. Takala. DSP formulation of a finite difference method for room acoustics simulation. In Proc. IEEE Nordic Signal Processing Symp. (NORSIG'96), pages 455-458, Espoo, Finland, Sept 1996b.

L. Savioja, T. J. Rinne, and T. Takala. Simulation of room acoustics with a 3-D finite difference mesh. In Proceedings of the International Computer Music Conference, pages 463-466, Aarhus, Denmark, 1994.

L. Savioja and V. Välimäki. Improved discrete-time modeling of multidimensional wave propagation using the interpolated digital waveguide mesh. In Proc. IEEE Int. Conf. Acoust., Speech, Signal Processing, volume 1, pages 459-462, April 1997. Munich, Germany.

L. Savioja and V. Välimäki. Reduction of the dispersion error in the triangular digital waveguide mesh using frequency warping. IEEE Signal Processing Letters, 6:58-60, 1999.

L. Savioja and V. Välimäki. Reducing the dispersion error in the digital waveguide mesh using interpolation and frequency-warping techniques. IEEE Trans. on Speech and Audio Processing, 8:184-194, 2000. 
L. Savioja and V. Välimäki. Multiwarping for enhancing the frequency accuracy of digital waveguide mesh simulations. IEEE Signal Processing Letters, 8:134-136, May 2001.

L. Savioja and V. Välimäki. Interpolated rectangular 3-d digital waveguide mesh algorithms with frequency warping. IEEE Trans. on Speech and Audio Processing, 11:783-790, Nov 2003.

T. Schetelig and R. Rabenstein. Simulation of three-dimensional sound propagation with multidimensional wave digital filters. In IEEE Int. Conf. on Acoustics, Speech \& Signal Processing (ICASSP), Seattle, USA, May 1998.

J. B. Schneider, C. L. Wagne, and S. L. Broschat. Implementation of transparent sources embedded in acoustic finite-difference time-domain grids. J. Acoust. Soc. Am., 103:136-142, Jan 1998a.

J. B. Schneider, C. L. Wagner, and R. J. Kruhlak. Simple conformal methods for FDTD modeling of pressure-release surfaces. J. Acoust. Soc. Am., 104(6):3219-3226, 1998b.

M. R. Schroeder. Computer models for concert hall acoustics. Am. J. Phys., 41:461-471, 1973.

S. Shelley and D. Murphy. Diffusion modeling at the boundary of a digital waveguide mesh. In Proc. of 13th European Signal Processing Conference, EUSIPCO2005, 2005a.

S. Shelley and D. T. Murphy. Measuring diffusion in a 2 d digital waveguide mesh. In Proc. of 8th International Conference on Digital Audio Effects (DAFx-05), pages 249-253, 2005b.

S. Shelley and D. T. Murphy. Modeling diffuse boundaries in the 2-d digital waveguide mesh. IEEE Trans. Audio, Speech and Language Processing, 16(3):651-665., 2008.

S. Siltanen, T. Lokki, S. Kiminki, and L. Savioja. The room acoustic rendering equation. J. Acoust. Soc. Am., 122(3):1624-1635, 2007.

J. O. Smith. Physical modelling using digital waveguides. Computer Music J., 16:74-91, 1992. 
J. O. Smith. Physical modeling synthesis update. Computer Music. J., 20: 44-56, 1996.

J. O. Smith. Applications of digital signal processing to audio and acoustics, chapter Principles of digital waveguide models of musical instruments, pages 417-466. Kluwer Academic Publisher, 1998.

J. O. Smith. On the equivalence of the digital waveguide and finite difference time domain schemes, 2004 URL http://www . citebase. org/abstract?id=oai : arXiv.org: physics/0407032.

M. Snir, S. W. Otto, S. Huss-Lederman, D. W. Walker, and J. Dongarra. MPI: The Complete Reference. MIT Press, 1995.

S. S. Soliman and M. D. Srinath. Continuous and Discrete Signals and Systems. Prentice Hall, 1997.

A. Southern and D. Murphy. 2nd order spherical harmonic spatial encoding of digital waveguide mesh room acoustic models. In Proc.s of the 10th Int. Conference on Digital Audio Effects (DAFx-07), pages 101-108, Bordeaux, France, 2007.

A. Southern and D. T. Murphy. A second order differential microphone technique for spatially encoding virtual room acoustics. In Proc. of 124th Audio Eng. Soc. Convention, Amsterdam, Holland, 2008.

Y. Srisukh, J. Nehrbass, F. L. Teixeira, J. F. Lee, and R. Lee. An approach for automatic grid generation in three-dimensional FDTD simulations of complex geometries. IEEE Antennas and Propagation Magazine, 44(4): 75-80, Aug 2002.

J. C. Strikwerda. Finite difference schemes and partial differential equations. Wadsworth \& Brooks, Pacific Grove, CA, 1989.

T. Su, Y. Liu, W. Yu, and R. Mittra. A new conformal mesh generating technique for conformal finite-difference time-domain (CFDTD) method. IEEE Antennas and Propagation Magazine, 46(1):37-49, Jan 2004.

D. M. Sullivan. A frequency-dependent FDTD method using Z transforms. IEEE Trans. on Antennas and Propagation, 40:1223-1230, 1992. 
H. Suzuki, A. Omotoy, and K. Fujiwaraz. Treatment of boundary conditions by finite difference time domain method. Acoust. Sci. \& Tech., 28(1):16$26,2007$.

U. P. Svensson. Modeling room acoustics. In Baltic-Nordic Acoustical Meeting, Mariehamn, Finland, 2004.

U. P. Svensson, R. I. Fred, and J. Vanderkooy. Analytic secondary source model of edge diffraction impulse responses. J. Acoust. Soc. Am., 106(5): 2331-2344, Nov 1999.

J. Szczupak and S. K. Mitra. Detection, localitation and removal of delayfree loops in digital filter structures. IEEE Trans. Acoustics, Speech and Signal Proc., 23(6):558-562, Dec 1975.

A. Taflove. Review of the formulation and applications of the finitedifference time-domain method for numerical modeling of electromagnetic wave interactions with arbitrary structures. Wave Motion, 10:547-582, 1988.

A. Taflove. Computational Electrodynamics: The Finite-Difference TimeDomain Method. Artech House, Norwood, MA, 1995.

A. Taflove. Advances in Computational Electrodynamics: The FiniteDifference Time-Domain Method. Artech House, Norwood, MA, 1998.

S. Taherzadeh and K. M. Li. On the turbulent jet noise near an impedance surface. J. Sound Vibration, 208(3):491-496, 1997.

Y. Takahashi, T. Otsuru, and R. Tomiku. In situ measurements of surface impedance and absorption coefficients of porous materials using two microphones and ambient noise. Appl. Acoust., 66:845-865, 2005.

C. K. W. Tam and J. C. Webb. Dispersion-relation-preserving finite difference schemes for computational acoustics. J. of Comput. Physics, 107: 262-281, 1992.

J. P. Thomas. An Investigation Of The Upwind Leapfrog Method For Scalar Advection And Acoustic/aeroacoustic Wave Propagation Problems. PhD thesis, University of Michigan, 1996. 
L. Trautmann and R. Rabenstein. Digital Sound Synthesis by Physical Modeling using the Functional Transformation Method. Academic Publishers, New York, USA, 2003.

N. Tsingos, I. Carlbom, G. Elko, R. Kubli, and T. Funkhouser. Validating acoustical simulations in the Bell Labs box. IEEE Computer Graphics and Applications, 22(4):28-37, Jul/Aug 2002.

K. R. Umashankar and A. Taflove. A novel method to analyze electromagnetic scattering of complex objects. IEEE Trans. Electromagn, Compat., 24:397-405, 1982.

V. Välimäki, J. Pakarinen, C. Erkut, and M. Karjalainen. Discrete time modeling of music instruments. Reports of Progress in Physics, 69:1-78, 2006 .

R. van der Geest and C. McChulloch. The virtual room: deriving acoustic characteristics by modelling. Proocedings of the Institute of Acoustics Reproduced Sound, 20:169-180, 1998.

M. Vetterli and J. Kovacevic. Wavelets and Subband Coding. Prentice Hall Signal Processing Series, 1995.

J. P. Vian and D. van Maercke. Calculation of the room response using a ray tracing method. In In Proc. of the ICA Symposium on Acoustics and Theater Planning for the Performing Arts, pages 74-78, 1986.

M. C. Vigeant, L. M. Wang, and J. H. Rindel. Differences in directional sound source behavior and perception between assorted computer room models. J. Acoust. Soc. Am., 116(4):2485, 2004.

C. L. Wagner and J. B. Schneider. An acoustic finite-difference time-domain algorithm with isotropic dispersion. J. of Comput. Acoustics, 13(2):365$384,2005$.

S. Wang. Finite-difference time-domain approach to underwater acoustic scattering problems. J. Acoust. Soc. Am., 99:1924-1931, 1996.

Z. Wang and S. F. Wu. Helmholtz equation least-squares method for reconstructing the acoustic pressure field. J. Acoust. Soc. Am., 102(4): 2020-2032, 1997. 
T. Whitted. An improved illumination model for shaded display. Communications of the ACM, 23(6):343-349, 1980.

E. G. Williams. Fourier Acoustics. Academic Press, London, 1999.

J. R. Wright. An exact model of acoustic radiation in enclosed spaces. $J$. Audio Eng. Soc., 43(10):813-820, 1995.

L. Wrobel and F. Aliabadi. The Boundary Element Method, Volume 1: Applications in Thermo-Fluids and Acoustics. John Wiley, New York, USA, 2002.

K. Yee. Numerical solution of initial boundary value problems involving Maxwell's equations in isotropic media. IEEE Trans. Antennas Propagat., 14:302-307, 1966.

A. Yefet and P. Petropoulous. A non-dissipative staggered fourth-order accurate explicit finite-difference scheme for the time-domain Maxwell's equations. Technical Report 99-30, ICASE, NASA, Langley Research Center, Hampton, 1999.

T. Yokota, S. Sakamoto, and H. Tachibana. Visualization of sound propagation and scattering in rooms. Acoust. Sci. \&6 Tech., 23(1):40-46, 2002.

X. Yuan, D. Broup, J. W. Wiskin, M. Berggren, R. Eidens, and S. A. Johnson. Formulation and validation of Berenger's PML absorbing boundary condition for FDTD simulation of acoustic scattering. IEEE Trans. on Ultrasonics, Ferroelectrics and Frequency Control, 44(4):816-822, July 1997.

A. Zayati. Numerische simulation dreidimensionaler schallfelder. Master's thesis, Chair of Multimedia Communications and Signal Processing, University of Erlangen-Nuremberg, 1998.

A. Zayati and R. Rabenstein. Sound field simulation by computational acoustics. Int. Journal of Adaptive Control and Signal Processing, 14: 663-680, 2000.

L. J. Ziomek. Fundamentals of Acoustic Field Theory and Space-Time Signal Processing. CRC Press, Boca Raton, 1995. 